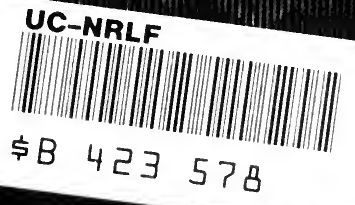



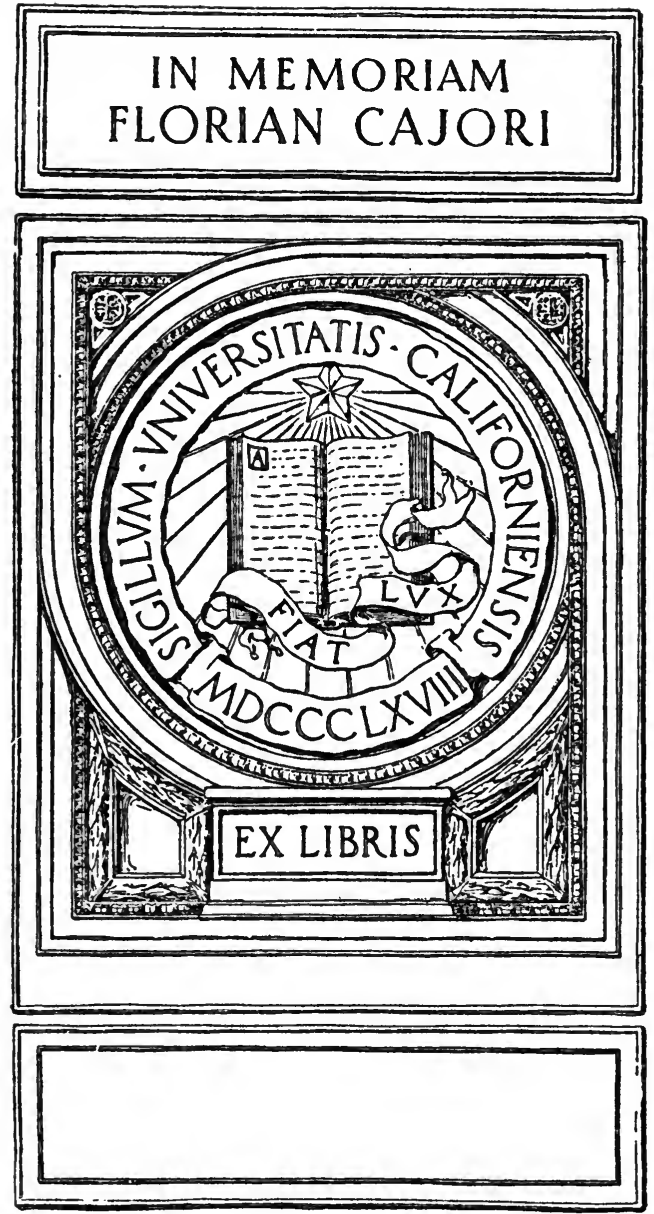
Th. Pajori 
Digitized by the Internet Archive in 2007 with funding from Microsoft Corporation 
A SHORT COURSE IN COLLEGE MATHEMATICS 


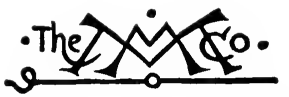

THE MACMILLAN COMPANY

NEW YORK - BOSTON - CHICAGO - DALLAS

ATLANTA - SAN FRANCISCO

\section{MACMILLAN \& CO., Limited \\ LONDON - BOMBAY - CALCUTTA \\ MELBOURNE}

THE MACMILLAN CO. OF CANADA, LrD. TORONTO 


\title{
A SHORT COURSE IN
}

\section{COLLEGE MATHEMATICS \\ COMPRISING}

THIRTY-SIX LESSONS

ON

\begin{abstract}
ALGEBRA, COÖRDINATE METHODS, AND
\end{abstract}
PLANE TRIGONOMETRY

BY

ROBERT E. MORITZ, Ph.D.

- PROFESSOR OF MATHEMATICS IN THE

UNIVERSITY OF WASHINGTON

X̛w 19ark

THE MACMILLAN COMPANY

1920

All rights reserved 
Copyright, 1919,

By THE MACMILLAN COMPANY.

Set up and electrotyped. Published November, rgrg.

F゙ormood 死ess

J. S. Cushing Co. - Berwick \& Smith Co.

Norwood, Mass., U.S.A. 


\section{PREFACE}

There has been an increasing demand in recent years for shorter courses in mathematics, based on the assumption, which our experience during the Great War has to some extent verified, that the ordinary processes of education may be greatly accelerated. Colleges of Engineering, Schools of Forestry, of Mines, of Architecture, and other schools of applied science are unwilling to give the time formerly allotted to the subject of mathematics, yet they insist that their students should be acquainted with every essential principle which is of actual use to them in their future work.

This little text is based on the supposition that such condensation and acceleration is possible. It was first prepared and printed for use in the mathematics classes of the Army and Navy Students Training Corps. The great emergency in which the nation found itself seemed to justify the attempt to take by direct assault a greater number of mathematical vantage points without stopping to explore all the intermediate terrain.

The book contains but thirty-six lessons, of which eighteen are given to the subject of trigonometry and the other eighteen to topics in algebra, to graphs, and to coördinate methods. Yet it is hoped that no essential principle of elementary trigonometry has been omitted, and that this subject has been treated with a completeness sufficient for the needs both of the engineer and the student of the more advanced branches of mathematics, such as analytical geometry and the calculus, while the familiarity gained in the use of coördinate methods should greatly facilitate the study of these latter subjects. 
If mathematics may be compared to a battle-fleet, this little book shows one of its vessels cleared for action. Additional guns have been mounted on the cleared deck, nothing remains on board but what is needed in the impending conflict.

In revising this text for after-war purposes little has been changed except the character of the applied problems which in the original text were largely chosen from the fields of military and naval science.

Robert E. Moritz.

The University of Washington, April, 1919. 


\title{
TABLE OF CONTENTS
}

\author{
CHAPTER I. ALGEBRA
}

LESSON

PAGE

1. Factoring . • . . . . . . . . . . . 1

2. Radicals, Fractional and Negative Fxponents $\quad$. 9

3. Imaginary Quantities . . . . • • . . . . 15

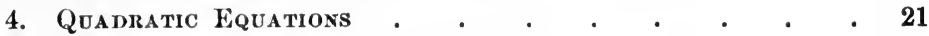

5. Applied Problems in Quadratic Equations . $\quad$. 29

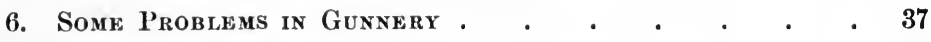

7. REVIEW . . . . . . . . . . . . 42

CHAPTER II. GRAPHIC METHODS

8. Coördinates and Simple Graphe . . . . . . . . 44

9. Related Grapis . . . . . . . • • . 56

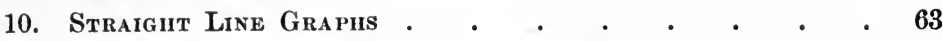

11. Simultaneous Straight Line Graphs • • • • • $\quad 68$

12. Curve Plotting $\quad$ •

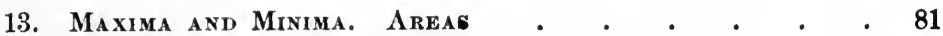

14. The Straight Line and Circle . . . . . . .90

15. Graphic Solution of Equations . . . . . . . 97

16. ReVIEW . . . . . . . . . . . . . 107

CHAITER III. TRIGONOMETRIC FUNCTIONS

17. The General Angle and Its Measures • • • 110

18. The Trigonometric or Circular Functions • • . 117

19. Reductions to the First Quadrant . • • . . . 121

20. Functions of an Acute Angle . . . . . . . . 128

21. Trigonometric Graphs . . . . . . . . . 134

22. Solution of Right Triangles . . . . . . . . 146 
23. Arithmetic Solution of Oblique Triangles • • • 155

24. Functions of the Sum and Difference of Two Angles . 162 25. Inverse Trigonometric Functions and Trigonometric

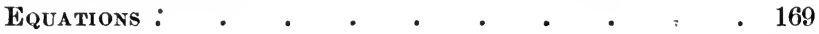
26. REVIEW . • . • . . . . . . . . . 176

CHAPTER IV. LOGARITHMS AND THEIR USE

27. Exponents and Logaritims . . • • • • . 179

28. Logarithmic Computation $\quad . \quad$.

29. Application of Logarithis to Mensuration of Plane

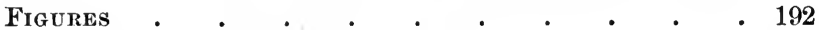

30. Logarithic and Exponential Curves . . . . . 195

CHAPTER V. LOGARITHMIC SOLUTION OF TRIANGLES

31. Oblique Triangles. Case I . • • • • • . 204

32. Oblique Triangles. Case II . . . • • • 210

33. Oblique Triangles. Case III . . . . . . . . 216

34. Oblique Triangles. Case IV . . . . . . . 221

35. Miscellaneous Problems Involving Triangles . • . 226

36. Review . . . . . . . . . • • . • . 231 


\section{GREEK LETTERS USED IN THIS TEXT}

$\begin{array}{clc}\text { Letter } & \text { Namr } & \text { English Equivalent } \\ \boldsymbol{\alpha} & \text { alpha } & \mathrm{a} \\ \beta & \text { beta } & \mathrm{b} \\ \boldsymbol{\epsilon} & \text { epsilon } & \mathrm{e} \\ \boldsymbol{\theta} & \text { theta } & \text { th } \\ \lambda & \text { lambda } & \mathrm{l} \\ \boldsymbol{\mu} & \mathrm{mu} & \mathrm{m} \\ \boldsymbol{\nu} & \mathrm{nu} & \mathrm{n} \\ \boldsymbol{\pi} & \mathrm{pi} & \mathrm{p} \\ \boldsymbol{\phi} & \mathrm{phi} & \mathrm{ph} \\ \boldsymbol{\omega} & \text { omega } & \mathrm{o}\end{array}$




\section{A SHORT COURSE IN MATHEMATICS}

\section{CHAPTER I}

\section{ALGEBRA \\ LESSON I-FACTORING}

1.1. Algebra, like arithmetic, deals with numbers. In arithmetic numbers are represented by figures, each figure or set of figures representing some specific number, while in algebra we use letters as well as figures to represent numbers, these letters representing any number whatsoever. For this reason the results arrived at in algebra are general results holding true for all numbers; in fact, for many purposes algebra may be looked upon as universal arithmetic.

We may illustrate the distinction between arithmetic and algebra by comparing the arithmetic relation $8^{2}-5^{2}=13 \times 3$ with the algebraic relation $a^{2}-b^{2}=(a+b)(a-b)$. The former tells us a fact about the numbers, $8,5,13$, and 3 , which helps us but little when dealing with other numbers, while the latter gives us an important relation between two numbers $a$ and $b$, no matter what $a$ and $b$ are.

1.2. Algebraic Symbols. The figures $1,2,3$, etc., and the other symbols such as $+,-, \times, \div,=,(),()^{2},()^{3}, \sqrt{ }$, etc., are used in algebra with the same meaning as in arithmetic. These together with letters to represent numbers, and certain other symbols that will be explained as we go along, are called algebraic symbols. Any collection of algebraic symbols which admits of interpretation is called an algebraic expression, in 
other words, an algebraic expression is some thought or idea expressed in algebraic language. $\left(a^{2}+b\right) / 2 c$ signifies that the number $b$ is to be added to the square of the number $a$ and that their sum is to be divided by two times the number c. The expression $x^{2}-3 x=5$ signifies that $x$ is a number which when multiplied by itself (squared) and the product diminished by three times the number the remainder is 5 . The algebraic expression $a^{2}-b^{2}=(a+b)(a-b)$ signifies that the difference of the squares of two numbers is always equal to the product of the sum of the numbers by their difference.

1.3. Monomial, Binomial, Trinomial, Polynomial. The parts of an algebraic expression connected by plus or minus signs are called its terms. If it consists of a single term the expression is called a monomial, if of two terms, a binomial, if of more than two terms, a polynomial. Polynomials having only three terms are also called trinomials.

$+x y, \frac{-4 x y}{3 c},-x^{2} \sqrt{ } a x,\left(a+b^{2}\right), x(y-z)$ are monomials.

$x+y, x^{2}-y^{2},(a+b)^{2}+(a-b)^{2}, x y-x z$ are binomials.

$x+y+z, \quad 3 x+4 y-3(x-y), \quad \frac{a+b}{a-b}+\frac{a-b}{a+b}-\frac{3 x+y-4 \sqrt{ } z}{5}$ are trinomials.

1.4. Integral and Rational. An algebraic expression is said to be integral when it does not contain letters in the denominator of any term. $x+4 y, \frac{x^{2}-5 a x+\frac{1}{2}}{3}, a b-\frac{1}{2} x^{2} y$ are integral expressions, $\frac{x}{y}, \frac{x^{2}+y}{a}, a^{2}-3 b+\frac{2}{c}$ are not.

An expression is said to be integral with respect to some particular letter when that letter does not occur in any denominator ; thus, $\frac{x^{2}}{a}+\frac{2 a x}{b}-\frac{1}{2}$ is integral in $x, \frac{1}{2} a x^{2}+\frac{4 y}{b}+\frac{1}{c}$ is integral in $a, x$, and $y$, but not in $b$ or $c$. 
An expression is said to be rational when it contains no letters under a radical sign; it is said to be rational with respect to some particular letter when that letter does not occur under a radical sign. $x^{2}+4 x+\sqrt{ } 3$ is rational while $x^{2}+\sqrt{ } x+1$ is not; $a^{2}-2 a \sqrt{ } b+x \sqrt{ } y$ is rational with respect to $a$ and $x$ but not with respect to $b$ or $y$.

Expressions which are not integral are said to be fractional, expressions which are not rational are irrational.

An expression which is both integral and rational is said to be integral rational.

In the preceding definitions it is assumed that the algebraic expressions have been reduced to their simplest form. $\frac{a^{2} b}{a}$ is not fractional, nor is $a^{2} \sqrt{b^{2}}$ irrational since, when reduced, the former is equal to $a b$ and the latter to $a^{2} b$.

1.5. Factoring. When two or more algebraic expressions are multiplied together, each of the cxpressions is said to be a factor of their product. By factoring an expression is meant the process of finding the factors which when multiplied together will produce the expression. For the present we shall consider only rational integral expressions; and by the factors of such an expression we shall understand the rational integral expressions which when multiplied together will yield the given expression.

Strictly speaking every expression contains itself and unity as factors since multiplying any number by 1 leaves the number unchanged; when we say that an expression cannot be factored we mean that it contains no integral rational factors except itself and 1.

$4 a^{2} b$ contains the factors $2,2, a, a$, and $b$, since $2 \times 2 \times a \times a \times b=$ $4 a^{2} b$. The factors of $3 a x^{2}-6 a^{2} x+9 a x$ are $3, a, x$, and $x-2 a+3$ since $3 \times a \times x \times(x-2 a+3)=3 a x^{2}-6 a^{2} x+9 a x$. The factors of $x^{2}-7 x+12$ are $x-4$ and $x-3$ since $(x-4)(x-3)=x^{2}-7 x+12$. 
$a-b$ has no integral rational factors except itself and 1 ; we therefore say that $a-b$ cannot be factored, even though $(\sqrt{ } a+\sqrt{ } b)(\sqrt{ } a-\sqrt{ } b)=$ $a-b$ as the student may readily verify by multiplication.

\subsection{Monomial Factors. When some letter is contained as} a factor in each term of an expression, that letter is a factor of the whole expression.

Type Form. $a k+b k+c k+a k=k(a+b+c+d)$.

Example 1. $3 x^{2}+4 a x-\frac{1}{2} x=x\left(3 x+4 a-\frac{1}{2}\right)$.

Example 2. $3(a+b)-(a+b)(c-d)=(a+b)(3-\overline{c-d})$

$$
=(a+b)(3-c+d) \text {. }
$$

\subsection{Factors of Binomials.}

(a) The difference of the same powers of two quantities always contains the difference of these quantities as a factor. The other factor can be found by division.

Type Form. $\quad \boldsymbol{a}^{\boldsymbol{n}}-\boldsymbol{b}^{\boldsymbol{n}}=(\boldsymbol{a}-\boldsymbol{b}) \boldsymbol{f}$, where the second factor $f$ can be found by dividing $a^{n}-b^{n}$ by $a-b$.

Example 1. $x^{3}-y^{3}=(x-y)\left(x^{2}+x y+y^{2}\right)$, where the second factor is the quotient of $x^{3}-y^{3}$ divided by $x-y$.

Example 2. $(a+2 b)^{2}-(a-3 b)^{2}$

$$
\begin{aligned}
& =[(a+2 b)-(a-3 b)][(a+2 b)+(a-3 b)] \\
& =5 b(2 a-b) .
\end{aligned}
$$

(b) The sum of the same odd powers of two quantities always contains the sum of these quantities as a factor. The other factor can be found by division.

Type Form. $\quad a^{2 n+1}+b^{2 n+1}=(a+b) f$, where the second factor $f$ can be found by dividing $a^{2 n+1}+b^{2 n+1}$ by $a+b$.

Example. $\quad x^{5}+y^{5}=(x+y)\left(x^{4}-x^{3} y+x^{2} y^{2}-x y^{3}+y^{4}\right)$, where the second factor can be found by dividing $x^{5}+y^{5}$ by $x+y$.

(c) The difference of the same even powers of two quantities always contains both the sum and the difference of these two quantities as factors. 
Type Form. $a^{2 n}-b^{2 n}=(a+b)(a-b) f$, where $f$ can be found by dividing $a^{2 n}-b^{2 n}$ by $a^{2}-b^{2}$.

Example. $x^{6}-y^{6}=(x+y)(x-y)\left(x^{4}+x^{2} y^{2}+y^{4}\right)$, where the last factor can be found by dividing $x^{6}-y^{6}$ by $x^{2}-y^{2}$.

(d) Special Cases, which should be memorized by the student.

$$
\begin{gathered}
a^{2}-b^{2}=(a-b)(a+b), \quad a^{3}-b^{3}=(a-b)\left(a^{2}+a b+b^{2}\right), \\
a^{3}+b^{3}=(a+b)\left(a^{2}-a b+b^{2}\right) .
\end{gathered}
$$

\subsection{Proof of the Theorems in 1.7.}

(a) To prove that $a^{n}-b^{n}$ is divisible by $a-b$.

Let us start out as if we were going to divide $a^{n}-b^{n}$ by $a-b$, thus

$$
\begin{gathered}
\frac{a-b\left|a^{n}-b^{n} \quad\right| a^{n-1}+}{a^{n}-a^{n-1} b} \\
a^{n-1} b-b^{n} \\
=b\left(a^{n-1}-b^{n-1}\right)
\end{gathered}
$$

This shows that after the first step in the division process the remainder is $b\left(a^{n-1}-b^{n-1}\right)$, therefore $a^{n}-b^{n}$ will be divisible by $a-b$ provided this remainder is; that is, provided $a^{n-1} \dot{-} b^{n-1}$ is divisible by $a-b$. By the same process of reasoning it follows that $a^{n-1}-b^{n-1}$ is divisible by $a-b$ provided $a^{n-2}-b^{n-2}$ is, and this in turn provided $a^{n-3}-b^{n-3}$ is. Proceeding in this way step by step we finally arrive at $a-b$, which we know is divisible by $a-b$. Therefore $a^{n}-b^{n}$ is divisible by $a-b$.

The student who fails to grasp the foregoing reasoning may find the following easier. The division process proves, as was shown, that $a^{n}-b^{n}$ is divisible by $a-b$ provided $a^{n-1}-b^{n-1}$ is. Let $n=2$, then $a^{2}-b^{2}$ is shown to be divisible by $a-b$ since $a-b$ is. Let $n=3$, then $a^{3}-b^{3}$ is shown to be divisible by $a-b$ since $a^{2}-b^{2}$ is. Let $n=4$, then $a^{4}-b^{4}$ is 
shown to be divisible by $a-b$ since $a^{3}-b^{3}$ is. Proceeding in this way we see that $a^{n}-b^{n}$ is divisible by $a-b$, no matter how large the exponent $n$ is.

This method of proof is called Mathematical Induction, or Complete Induction, or the proof from $n$ to $n+1$.

(b) To prove that $a^{2 n+1}+b^{2 n+1}$ is divisible by $a+b$. This may be proved by the same process as (a).

(c) To prove that $a^{2 n}-b^{2 n}$ is divisible by both $a+b$ and $a-b$.

Proof. We may write $a^{2 n}-b^{2 n}$ in the equivalent form $\left(a^{2}\right)^{n}-\left(b^{2}\right)^{n}$. By $(a)\left(a^{2}\right)^{n}-\left(b^{2}\right)^{n}$ is divisible by $a^{2}-b^{2}$, which is equal to $(a+b)(a-b)$.

\subsection{Factors of Trinomials.}

(a) Type Form. $a^{2}+\mathbf{2} a b+b^{2}=(a+b)(a+b)=(a+b)^{2}$.

Example. $(x+y)^{2}+2(x+y)(2 x-3 y)+(2 x-3 y)^{2}$

$$
=[(x+y)+(2 x-3 y)]^{2}=(3 x-2 y)^{2} \text {. }
$$

(b) Type Form. $\boldsymbol{a}^{2}-\mathbf{2} \boldsymbol{a b}+\boldsymbol{b}^{2}=(\boldsymbol{a}-\boldsymbol{b})(\boldsymbol{a}-\boldsymbol{b})=(\boldsymbol{a}-\boldsymbol{b})^{2}$.

Example. $25(m+n)^{2}-10(m+n)(m-n)+(m-n)^{2}$

$$
=[5(m+n)-(m-n)]^{2}=(4 m+6 n)^{2}=4(2 m+3 n)^{2} .
$$

(c) Type Form. $x^{2}+(a+b) x+a b=(x+a)(x+b)$.

Example. Find the factors of $x^{2}+7 x+12$. Here $a+b=7$ and $a b=12$, hence to find the factors we must find first two numbers whose product is 12 and whose sum is 7 . The factors of 12 are 1 and 12 , or 2 and 6 , or 3 and 4 . Since the sum of the numbers must be 7 , the numbers are 3 and 4. Hence the factors of $x^{2}+7 x+12$ are $x+3$ and $x+4$.

Example. Find the factors of $p^{2}-p-42$. Here $a+b=-1$, and $a b=-42$, hence to find the factors we must first find two numbers whose product is -42 and whose sum is -1 . By trying the various factors into which -42 can be factored we find that the required numbers are -7 and +6 . Hence the factors of $p^{2}-p-42$ are $p-7$ and $p+6$.

1.10. The Factor Theorem. Any algebraic expression which is integral and rational with respect to $x$ and reduces to zero when $x$ is replaced by $k$ contains $x-k$ as a factor. 
Proof. Since the expression is integral in $x$ it cannot contain $x$ in the denominator and since it is rational in $x$ it cannot contain $x$ under a radical sign. It can therefore be simplified and arranged in ascending powers of $x$. If we denote the expression by $f(x)$ we may therefore write it in the form

$$
f(x)=a+b x+c x^{2}+d x^{3}+e x^{4}+\text { etc., }
$$

where $a, b, c, d$, etc., are the coefficients of the various powers of $x$. If now we replace $x$ by $k$ we obtain

$$
f(k)=a+b k+c k^{2}+d k^{3}+e k^{4}+\text { etc. }=0 \text { (by hypothesis). }
$$

Subtracting the second expression from the first we have

$$
f(x)=0+b(x-k)+c\left(x^{2}-k^{2}\right)+d\left(x^{3}-k^{3}\right)+e\left(x^{4}-k^{4}\right)+\text { etc. }
$$

Now by 1.7 each of the binomials $x-k, x^{2}-k^{2}, x^{3}-k^{3}$, etc., contains $x-k$ as a factor, hence by $1.6 x-k$ is a factor of the whole expression $f(x)$.

Example. Show that $x^{3}+3 x^{2}-4 x-12$ contains $x-2$ and $x+3$ as factors. Replacing $x$ by 2 we have $2^{3}+3 \cdot 2^{2}-4 \cdot 2-12=8+12-8$ $-12=0$; hence $x-2$ is a factor. Again replacing $x$ by -3 we have $(-3)^{3}+3(-3)^{2}-4(-3)-12=-27+27+12-12=0$; hence $x+3$ is also a factor.

Example. Show that $(x-a)^{3}+(a-b)^{3}+(b-x)^{3}$ contains $x-b$ as a factor. Replacing $x$ by $b$ we have $(b-a)^{3}+(a-b)^{3}+(b-b)^{3}=0$ since $b-b=0$ and $(b-a)^{3}=-(a-b)^{3}$; hence $x-b$ is a factor. In the same way it may be shown that $x-a$ and $a-b$ are factors of the given expression.

\section{EXERCISE 1}

Find all the rational integral factors of the following algebraic expressions :

1. $a b c d+b^{2} c^{2} d-3 a c d^{2}-3 c^{2} d$.
2. $x^{2}+3 x y$.
5. $p(a+b)-a-b$.
3. $x y^{2}-3 a b x y+\frac{1}{2} x^{2} y^{2}$.
6. $a^{2}-1$.
4. $a(x-y)+b(x-y)$.
7. $a^{4}-b^{4}$. 
8. $(a-b)^{2}-c^{2}$.

9. $t^{3}-1$.

10. $t^{3}+1$.

14. $9+6 m+m^{2}$.

16. $x^{2}-x-12$.

17. $x^{2}+x-12$.

18. $x^{2}+2 x-15$.

19. $y^{2}+8 y+15$.

20. $x^{2} y^{2}-2 x y-15$.
11. $(3-x)^{2}-(3+x)^{2}$.

12. $a x-a-x+1$.

13. $m^{2}-6 m+9$.

15. $4(x+3)^{2}-12 y^{2}(x+3)+9 y^{4}$.

21. $x^{2}-8 x y+15 y^{2}$.

22. $m^{2}+2 m-24$.

23. $p^{2}-10 p-24$.

24. $a^{4}-5 a^{2}-24$.

25. $a^{4}+23 a^{2} b^{2}-24 b^{2}$.

26. $(a+b)^{2}-7(a-b)(c+d)+12(c+d)^{2}$.

27. $\frac{x^{4}}{16}+\frac{x^{2} y^{2}}{6}+\frac{y^{4}}{9}$.

28. $x^{4}+x^{3}+x^{2}+x$.

29. Show that $a-b$ is a factor of $a^{4}\left(b^{2}-c^{2}\right)+b^{4}\left(c^{2}-a^{2}\right)+$ $c^{4}\left(a^{2}-b^{2}\right)$, and find two other factors.

30. Find three factors of $a(b-c)^{3}+b(c-a)^{3}+c(a-b)^{3}$.

31. Show that $x-a$ and $x-b$ are factors of the expression $x^{2}-a^{2} \frac{(x-b)(x-c)}{(a-b)(a-c)}-b^{2} \frac{(x-c)(x-a)}{(b-c)(b-a)}$. Is $x-c$ a factor?

32. $a^{2}$ may be represented by a square whose side is $a, a b$ by a rectangle whose sides are $a$ and $b$ respectively. By drawing a square whose side is $(a+b)$ and partitioning it, it is at once apparent that $(a+b)^{2}=a^{2}+2 a b+b^{2}$. Similarly show by drawing a diagram that

$$
(x+a)(x+b)=x^{2}+(a+b) x+a b .
$$

33. Follow the suggestion in problem 32 and show by means of a diagram that $a(b+c+d)=a b+a c+a d$. Similarly show that $a^{2}-b^{2}=(a+b)(a-b)$.

34. Prove by mathematical induction the formula in 1.7, $(b)$. 


\section{LESSON II - RADICALS, FRACTIONAL AND NEGATIVE EXPONENTS}

2.1. Square-root, Cube-root, $\boldsymbol{n}$ th-root. By the square-root of a number $a(\sqrt{ } a)$ is meant the number whose square is $a$; by the cube-root of $a(\sqrt[3]{a})$ the number whose cube is $a$; by the $n$ th-root of $a(\sqrt[n]{a})$ the number whose $n$th power is $a$.

Thus $\sqrt{ } 9= \pm 3$, that is either +3 or -3 , since $(+3)^{2}=9$ and $(-3)^{2}=9 . \quad \sqrt[3]{8}=+2$ since $(+2)^{3}=8$, and $\sqrt[3]{-8}=-2$ since $(-2)^{3}=-8$. An indicated root such as $\sqrt[3]{2}$ or $\sqrt{ } 8$ is called a radical.

2.2. Assuming that all square-roots are numbers it follows from the above that:

Every number is the square of its own square-root ;

Every number is the square of some other number.

Similar statements may be made for the cube-root, fourthroot, $n$ th-root.

Example. 2 is the square of $\sqrt{ } \mathbf{2}$ or of $1.414 \ldots, 2$ is also the cube of $\sqrt[3]{2}$ or of $1.259 \cdots \quad \frac{1}{3}$ is the square of $\sqrt{\frac{1}{3}}$ or of $0.577 \ldots \quad \frac{1}{3}$ is also the cube of $\sqrt[8]{\frac{1}{3}}$ or of $0.693 \ldots$.

\subsection{Fundamental Laws of Radicals.}

(a) The square-root of a product is equal to the product of the square-roots of its factors, that is $\sqrt{a b c} \cdots=\sqrt{ } a \cdot \sqrt{ } b \cdot \sqrt{ } c \cdots$.

Example. $\quad \sqrt{ } 50=\sqrt{25 \times 2}=\sqrt{ } 25 \times \sqrt{ } 2=5 \sqrt{ } 2$,

$$
\sqrt{a^{2} x^{2} y}=\sqrt{a^{2}} \sqrt{x^{2}} \sqrt{ } y=a x \sqrt{ } y .
$$

(b) The square-root of a fraction is equal to the square-root of the numerator divided by the square-root of the denominator, that is, $\sqrt{ }(a / b)=\sqrt{ } a / \sqrt{ } b . \quad \sqrt{ }(1 / a)=\sqrt{ } 1 / \sqrt{ } a=1 / \sqrt{ } a$.

Example. $\sqrt{\frac{3}{4}}=\frac{\sqrt{ } 3}{\sqrt{ } 4}=\frac{\sqrt{ } 3}{2}=\frac{1}{2} \sqrt{ } 3, \sqrt{\frac{a^{2} b}{x^{2}}}=\frac{\sqrt{a^{2}} \sqrt{ } b}{\sqrt{x^{2}}}=\frac{a \sqrt{ } b}{x}=\frac{a}{x} \sqrt{ } b$.

These laws hold for any root as well as for square-roots. 
(c) The mith-root of the nth-root of any number is equal to the mnth-root of the number, that is $\sqrt[m]{\sqrt[n]{ } a}=\sqrt[m n]{a}$; for instance, $\sqrt[3]{ } \sqrt{ } a=\sqrt[6]{ } a$.

(d) The mth-root of a number is equal to the mnth-root of the nth power of that number, that is $\sqrt[m]{ } a=\sqrt[m n]{a^{n}}$; for instance, $\sqrt{ } a=\sqrt[6]{a^{3}}=\sqrt[8]{a^{4}}=\sqrt[10]{a^{5}}$.

This law enables us to reduce radicals having different indices to radicals having the same index; thus $\sqrt{ } a, \sqrt[3]{ } b, \sqrt[5]{ } c$ are respectively equal to $\sqrt[30]{a^{15}}, \sqrt[30]{b^{10}}, \sqrt[30]{c^{6}}$. We are thus enabled to multiply radicals having different indices by first reducing them to radicals having the same index.

Example. $\quad \sqrt{ } a \sqrt[3]{b} \sqrt[5]{ } c=\sqrt[30]{a^{16}} \sqrt[30]{b^{10}} \sqrt[30]{c^{6}}=\sqrt[30]{a^{15} b^{10} c^{6}}$ by $(a)$.

2.4. The Laws of Exponents. It is customary to write $a^{2}$ for $a \times a, a^{3}$ for $a \times a \times a, a^{4}$ for $a \times a \times a \times a$, and $a^{n}$ for the product of $n$ factors each equal to $a$. The exponent $n$ indicates the number of factors which are multiplied together and must therefore be some integer (whole number like 1, 2, 3, 4, etc.). From this definition of an exponent it follows immediately that

(a) $a^{m} \times a^{n}=a^{m+n}$. This is known as the Fundamental Index Law. From (a) combined with the ordinary rules of multiplication it follows that also $(b) a^{n} \times b^{n}=(a b)^{n}$, and (c) $\left(a^{m}\right)^{n}=a^{m n}$.

Examples. $2^{3} \times 2^{4}=2^{7}, 2^{5} \times 3^{5}=6^{5},\left(2^{3}\right)^{4}=2^{12}$.

2.5. Fractional Exponents. What is the meaning of a fractional exponent such as $\frac{1}{2}$ in the expression $a^{\frac{1}{2}}$ ? We may say that thus far it has no meaning since every exponent as above defined is necessarily a whole number. Since it has no meaning we may therefore give it any meaning we wish, in particular we may choose this meaning in accordance with the index law $(a)$. That is to say the meaning of $a^{\frac{1}{2}}$ is to be 
such that $a^{\frac{1}{2}} \times a^{\frac{1}{2}}=a^{\frac{1}{2}+\frac{1}{2}}=a^{1}=a$, from which it follows that $a^{\frac{1}{2}}=\sqrt{ } a$.

In the same way we may define $a^{\frac{1}{3}}$ in such a way that $a^{\frac{1}{3}} \times a^{\frac{1}{3}} \times a^{\frac{1}{3}}=a^{\frac{1}{3}+\frac{1}{3}+\frac{1}{3}}=a^{1}=a$, from which it follows that $a^{\frac{1}{3}}=\sqrt[3]{a}$.

Similarly we conclude that $a^{\frac{1}{n}}$ means the nth-root of $a$.

To find a meaning for $a^{\frac{m}{n}}$ where $m$ and $n$ are both positive, we have from the index law $a^{\frac{m}{n}} \times a^{\frac{m}{n}} \times a^{\frac{m}{n}} \times \cdots$ to $n$ factors $=\left(a^{\frac{m}{n}}\right)^{n}=a^{m}$, hence $a^{\frac{m}{n}}=\sqrt[n]{a^{m}} ;$ also since $\left(a^{\frac{1}{n}}\right)^{m}=a^{\frac{m}{n}}$ and $a^{\frac{1}{n}}=\sqrt[n]{ } a$, we have $a^{\frac{m}{n}}=(\sqrt[n]{a} a)^{m}$, so that $a^{\frac{m}{n}}=\sqrt[n]{a^{m}}=(\sqrt[n]{ } a)^{m}$.

The foregoing results may be stated in words as follows:

$a^{\frac{m}{n}}$ is the nth-root of the mth power of $a$; it is also the mth power of an nth-root of $a . *$

This is a direct consequence of the universality of the index law as are indeed all the fundamental laws of radicals mentioned in $\mathbf{2 . 3}$.

2.6. The Meaning of $a^{0}$. If zero is used as an exponent it should satisfy the index law. This requires that $a^{0} \times a^{n}=$ $a^{0+n}=a^{n}$, that is, $a^{0}=\frac{a^{n}}{a^{n}}=1$.

$a$ is any number different from zero; we may therefore say :

Any number different from zero when raised to a power whose exponent is zero equals unity.

2.7. Negative Exponents. The method pursued in 2.5 and 2.6 likewise enables us to assign a meaning to a negative exponent.

* The results of this section are true without exception only for the positive values of the roots. 
By the index law we have $a^{-n} \times a^{n}=a^{-n+n}=a^{0}=1$; therefore, $a^{-n}=\frac{1}{a^{n}}$, in words,

By $a^{-n}$ we mean the reciprocal of $a^{n}$.

It follows immediately that $\frac{1}{a^{-n}}=\frac{1}{\left(1 / a^{n}\right)}=a^{n}$.

Therefore, any factor may be transferred from the numerator to the denominator of a fraction, or from the denominator to the numerator, by merely changing the sign of its exponent. It must be remembered that when there is no exponent expressed the exponent 1 is understood.

Example. $a^{-1}=1 / a, 1 / a^{-1}=a, \frac{a^{2} b^{-3} c^{4} x^{-1} y^{0}}{a^{-1} b^{2} c^{2} x^{-3}}=\frac{a^{2} a^{1} c^{4} x^{3}}{b^{2} b^{3} c^{2} x}=\frac{a^{3} c^{2} x^{2}}{b^{5}}$.

2.8. The Use of Fractional Exponents. Fractional exponents enable us to work with radicals as readily as with expressions free from radicals. All we need to do is to express the radicals by means of fractional exponents after which we can apply the index law to perform whatever operations are called for.

Examples. (a) Multiply together the radicals $\sqrt[3]{a^{4}} \sqrt[5]{b^{3}} \sqrt[3]{a^{2}} \sqrt[5]{b^{12}}$.

Solution. $\quad \sqrt[3]{a^{4}}=a^{\frac{4}{3}}, \sqrt[5]{b^{3}}=b^{\frac{3}{5}}, \sqrt[3]{a^{2}}=a^{\frac{2}{3}}, \sqrt[5]{b^{12}}=b^{\frac{12}{5}}$,

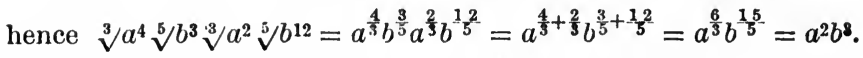

(b) Divide $\sqrt{(a+b)} \sqrt[3]{x^{2}+y^{2}}$ by $\sqrt[3]{a+b} \sqrt[5]{x^{2}+y^{2}}$.

Solution. $\quad \sqrt{a+b}=(a+b)^{\frac{1}{2}}, \quad \sqrt[3]{x^{2}+y^{2}}=\left(x^{2}+y^{2}\right)^{\frac{1}{3}}$,

$$
\sqrt[3]{a+b}=(a+b)^{\frac{1}{3}}, \quad \sqrt[5]{x^{2} y^{2}}=\left(x^{2} y^{2}\right)^{\frac{1}{5}} .
$$

Hence

$$
\begin{aligned}
\frac{\sqrt{a+b} \sqrt[3]{x^{2}+y^{2}}}{\sqrt[3]{a+b} \sqrt[5]{x^{2}+y^{2}}} & =\frac{(a+b)^{\frac{1}{2}}\left(x^{2}+y^{2}\right)^{\frac{1}{3}}}{(a+b)^{\frac{1}{3}}\left(x^{2}+y^{2}\right)^{\frac{1}{5}}} \\
& =(a+b)^{\frac{1}{2}-\frac{1}{3}}\left(x^{2}+y^{2}\right)^{\frac{1}{3}-\frac{1}{5}}
\end{aligned}
$$$$
=(a+b)^{\frac{1}{6}}\left(x^{2}+y^{2}\right)^{\frac{2}{15}}
$$$$
=\sqrt[6]{(a+b)} \sqrt[15]{\left(x^{2}+y^{2}\right)^{2}} .
$$

(c) Reduce the radicals $\sqrt{a^{3} b}, \sqrt[3]{a^{2} b^{4}}, \sqrt[5]{a b^{2} c^{3}}$ to radicals having a common index. 
Solution. $\quad \sqrt{a^{3} b}=\left(a^{3} b\right)^{\frac{1}{2}}=\left(a^{3} b\right)^{\frac{1}{3} \frac{5}{6}}=\sqrt[30]{\left(a^{3} b\right)^{15}}=\sqrt[30]{a^{45} b^{15}}$.

$$
\begin{gathered}
\sqrt[3]{a^{2} b^{4}}=\left(a^{2} b^{4}\right)^{\frac{1}{3}}=\left(a^{2} b^{4}\right)^{\frac{1}{3} 0}=\sqrt[30]{\left(a^{2} b^{4}\right)^{10}}=\sqrt[30]{a^{20} b^{40}} \\
\sqrt[5]{a b^{2} c^{3}}=\left(a b^{2} c^{3}\right)^{\frac{1}{5}}=\left(a b^{2} c^{3}\right)^{\frac{6}{30}}=\sqrt[30]{\left(a b^{2} c^{3}\right)^{6}}=\sqrt[30]{a^{6} b^{12} c^{18}} .
\end{gathered}
$$

2.9. The Use of Negative Exponents. One of the important uses of negative exponents is to expedite the writing and especially the printing of fractions. It is easier to write and much easier to print $4 \times 10^{-2}$ than $\frac{4}{10^{2}}$ and $(x+y)^{-4}$ than $\frac{1}{(x+y)^{4}}$.

\section{EXERCISE 2}

1. The so-called dimensions of the physical quantities velocity, acceleration, force, work, and pressure are $L T^{-1}$, $L T^{-2}, L M T^{-2}, L^{2} M T^{-2}$, and $L^{-1} M T^{-2}$ respectively, where $L$ stands for length, $\boldsymbol{T}$ for time, and $\boldsymbol{M}$ for mass. Write these expressions without using negative exponents.

2. The following expressions represent ratios of certain electrostatic units :
(a) $\frac{M L^{2} T^{-2}}{M^{\frac{1}{2}} L^{\frac{3}{2}} T^{-1} K^{\frac{1}{2}}}$
(b) $\frac{M^{\frac{1}{2}} L^{\frac{3}{2}} T^{-1} K^{\frac{1}{2}}}{M^{\frac{1}{2}} L^{\frac{1}{2}} T^{-1} K^{-\frac{1}{2}}}$.

Simplify these expressions and write the results in integral form.

3. The following are ratios between certain electromagnetic units :
(a) $I^{-1} T^{2} P^{-1}$,
(b) $M^{\frac{1}{2}} L^{-\frac{1}{2}} T^{-1} P^{-\frac{1}{2}}$,
(c) $M^{\frac{1}{2}} L^{\frac{3}{2}} T^{-2} P^{\frac{1}{2}}$.

Express these ratios using neither negative nor fractional exponents.

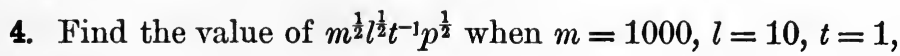
- and $p=1$

5. The factor required to convert electrical potential from millimeter-milligramme-second units to c.g.s. units is $m^{\frac{1}{2}} l^{\frac{1}{2}} t^{-1} k^{-\frac{1}{2}}$; 
find its numerical value when $m=0.001, l=0.1, t=1$, and $k=1$.

6. The factor required to convert intensity of magnetic field from foot-grain-minute units to c.g.s. units is $m^{\frac{1}{2}} l^{-\frac{1}{2}} t^{-1} p^{-\frac{1}{2}}$. Find its numerical value when $m=0.0648, l=30.48, t=60$, and $p=1$. Ans. 0.00076847 .

Simplify the following expressions :
7. $\sqrt{ } 72$.
11. $\sqrt{a^{2} b^{3} x^{4} y}$.
8. $\sqrt{ } \frac{2}{3}$.
12. $a^{5} b^{4} \times a^{-4} b^{-2} c^{-1}$.
14. $\left(\frac{a}{x}\right)^{-1}$.
9. $\sqrt[3]{\frac{2}{3}}$.

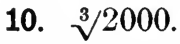
13. $\sqrt[3]{\frac{125(a+b)^{4}}{128(x+y)^{6}}}$.
15. $\left(\frac{a^{2}}{x^{2}}\right)^{-\frac{1}{2}}$.

16. Multiply $x^{\frac{2}{3}}+x^{\frac{1}{3}} y^{\frac{1}{3}}+y^{\frac{2}{3}}$ by $x^{\frac{1}{3}}-y^{\frac{1}{3}}$.

17. Divide $a-x$ by $a^{\frac{1}{2}}-x^{\frac{1}{2}}$.

18. Simplify $\left(x^{\frac{1}{4}}+y^{\frac{1}{4}}\right)\left(x^{\frac{1}{4}}-y^{\frac{1}{4}}\right)\left(x^{\frac{1}{2}}+y^{\frac{1}{2}}\right)$.

19. Simplify $\left(x^{\frac{2}{3}}-y^{-\frac{1}{3}}\right)^{2}$.

20. Reduce to its simplest form $\sqrt[3]{\sqrt{a^{5} b^{4}}} \times \sqrt{a \sqrt{a b}} \times \sqrt{a \sqrt[3]{a b}} . \quad$ Ans. $a^{2} b \sqrt[4]{a} \sqrt[12]{b}$. 


\section{LESSON III - IMAGINARY NUMBERS}

\subsection{Fundamental Laws of Arithmetic and Algebra. The}

numbers used in counting a group or collection of things are called natural numbers, whole numbers, or positive integers. An important property of these numbers is that among them there is no greatest number, that is, for every number that may be mentioned there are numbers (in fact an endless number of numbers) which are greater. This fact is expressed by saying that the number of integers is infinite (in = without, finis $=$ end).

The fundamental operation of arithmetic is counting. Counting is expedited by certain processes known as addition, multiplication, and powering (or involution). These processes are subject to the following laws known as the fundamental laws of arithmetic (and algebra):

(a) $a+b=b+a$, (Commutative law of addition)

(b) $a+b+c=a+(b+c)$, (Associative law of addition)

(c) $a \times b=b \times a$, (Commutative law of multiplication)

(d) $a \times b \times c=a \times(b \times c)$, (Associative law of multiplication)

(e) $a(b+c)=a b+a c$, (Distributive law)

(f) $a^{m} \times a^{n}=a^{m+n}, a^{n} \times b^{n}=(a b)^{n},\left(a^{m}\right)^{n}=a^{m n}$, (Index laws).

All these laws follow at once from the assumption that the number of things in a collection is independent of the order of counting.

3.2. Inverse Processes. Each of the direct processes addition, multiplication, and powering, suggests another known as its inverse.

The inverse of addition is subtraction, the inverse of multi- 
plication is division, the inverse of powering (or involution) is root-extraction (or evolution). These inverse processes are defined as follows:

$a-b$ is that number which when added to $b$ gives $a$;

$a \div b$ is that number which when multiplied by $b$ gives $a$;

$\sqrt[b]{ } a$ is that number which when powered by $b$ gives $a$.

3.3. Classification of Numbers. Since the combination of any two or more collections of things forms a single collection it follows at once that any two numbers combined into a sum, product, or power will, without exception, yield a third number. We express this fact by saying that addition, multiplication, and powering with positive integers is always permissible or possible.

The situation is different when we consider subtraction. Considering positive integers only, is $a-b$ always possible? Suppose $a=3$ and $b=7$, then $3-7$ calls for a number which when added to 7 gives 3 . Clearly there is no positive integer which satisfies this demand, and we would have to say that $a-b$ is impossible unless $a$ is equal to or greater than $b$. To avoid this we call $a-b$ a number even if $a$ is less than $b$, and to distinguish such numbers from positive integers we call them negative numbers.

Negative numbers originate from subtraction when the minuend does not exceed the subtrahend.

Similarly, fractions arise in the attempt to make division always possible. If we consider no numbers other than integers division is possible only when the dividend is an exact multiple of the divisor. $3 \div 4$ calls for a number which when multiplied by 4 yields 3 . Evidently there exists no integer satisfying this demand. Nevertheless we consider $\frac{3}{4}$, which does satisfy this demand, a number, but call it a fraction to distinguish it from an integer. We thus see that 
Fractions originate from division when the dividend is not an exact multiple of the divisor.

A third set of new numbers arises from root-extraction. $\quad \sqrt{ } 4$ asks for a number whose square is 4 and the number 2 satisfies this demand. But how about $\sqrt{ }^{2}$ ? There is no integer whose square is 2 , neither is there any fraction whose square is 2 . The only way out of the difficulty is to consider $\sqrt{ }^{2}$ a new number. Similarly the square-root of any number which is not a perfect square, the cube-root of any number which is not a perfect cube, the fourth root of any number which is not a perfect fourth power, all of these lead to new numbers which are neither integers nor fractions. We call these new numbers irrational numbers, to distinguish them from integers and fractions which together are called rational numbers.

Irrational numbers originate from the extraction of the nthroot of a number which is not a perfect nth power of some other number.

Root-extraction leads to still another set of new numbers. Consider $\sqrt{-4}$. This calls for a number whose square is -4. Neither +2 nor -2 satisfies this demand, for $(+2)^{2}=$ +4 and $(-2)^{2}=+4$ and not -4 . Neither is there any fraction or any irrational number whose square is -4 . As in the preceding cases, we have a choice between saying that $\sqrt{-4}$ is impossible and saying that $\sqrt{-4}$ itself is a number though different from all those previously considered. In the same way every square-root of a minus number leads to a new number, and not only every square root but every even root of a minus number leads to a new number of the same kind as $\sqrt{-4}$. We call these new numbers imaginary numbers to distinguish them from all the preceding, - integral, negative, fractional, and irrational, which together are called real numbers. 
Imaginary numbers arise in extracting an even root of a negative number.

3.4. Algebraic and Transcendental Numbers. All the numbers mentioned in the preceding section taken together belong to a class of numbers known as algebraic numbers. Every number found in solving an algebraic equation is an algebraic number and every algebraic number satisfies some algebraic equation. There are other numbers which cannot be found by solving algebraic equations. They are called transcendental numbers. The number $\pi=3.14159 . .$. , which is the ratio of the circumference of a circle to its diameter, is such a number.

3.5. Imaginary Numbers. It may be shown that all numbers, imaginary as well as real, are subject to all the fundamental laws enumerated in 3.1 ; in fact it is this observation, namely that they may be treated as are natural numbers, that entitles them to be called numbers. By the index law $\sqrt{-4}$ $=\sqrt{ } 4 \times \sqrt{-1}$, similarly every imaginary number as $\sqrt{-a}=$ $\sqrt{ } a \times \sqrt{-1}$, that is, every imaginary number may be considered the product of some real number multiplied by $\sqrt{-1}$. For convenience $\sqrt{-1}$ is universally denoted by the letter $i$, so that every imaginary number may be written $a i$, where $a$ is some real number and $i=\sqrt{-1}$.

Now by definition $(\sqrt{-1})^{2}=-1$, that is $i^{2}=-1$, and therefore $i^{4}=i^{2} \times i^{2}=-1 \times-1=+1, i^{6}=i^{4} \times i^{2}=1 \times-1=-1$, $i^{8}=i^{4} \times i^{4}=1 \times 1=+1$, that is,

Every even power of $\sqrt{-1}$ is equal to +1 or $-1,+1$ if the exponent is a multiple of $4,-1$ if it is not.

Again, $i^{3}=i^{2} \times i=-1 \times i=-i, i^{5}=i^{4} \times i=+1 \times i=+i$, $\imath^{7}=i^{6} \times i=-1 \times i=-i$, etc., that is,

Every odd power of $\sqrt{-1}$ is equal to $+i$ or $-i,+i$ if the exponent is a multiple of 4 increased by $1,-i$ if it is not. 
3.6. Complex Numbers. The sum or difference of a real number and an imaginary number is called a complex number. Since every imaginary number can be expressed as the product of some real number times $i$ it follows that every complex number is of the form $a+b i$, or $a-b i$, or by the form $a+b i$ alone, since $b$ may be negative as well as positive. Complex numbers, like real or imaginary numbers, are subject to all the fundamental laws and may therefore be added, subtracted, multiplied, and divided like other numbers.

Examples :

(a) Find the sum of $5+4 i$ and $3+2 i$. Ans. $8+6 i$ or $8+6 \sqrt{-1}$.

(b) Find the difference between $5+4 i$ and $3+2 i$. Ans. $2+2 i$.

(c) Multiply $5+3 i$ by $3-2 i$.

Solution. $\quad(5+3 i)(3-2 i)=15+9 i-10 i-6 i^{2}$

$$
=15-i-6(-1)=21-i \text {. }
$$

(d) Simplify the expression $(5-3 i)^{2}$.

Solution. $\quad(5-3 i)^{2}=5^{2}-2 \times 5 \times 3 i+9 i^{2}=25-30 i-9=16-30 i$.

(e) Multiply $5-3 i$ by $5+3 i$.

Solution. $(5-3 i)(5+3 i)=5^{2}-9 i^{2}=25+9=34$.

(f) Simplify $\frac{5-3 i}{2+3 i}$.

Solution. Multiply both the numerator and denominator by $2-3 i$, thus

$$
\frac{(5-3 i)(2-3 i)}{(2+3 i)(2-3 i)}=\frac{10-6 i-15 i+9 i^{2}}{4-9 i^{2}}=\frac{1-21 i}{13} \text {. }
$$

(g) Simplify $\frac{5-3 i}{2-3 i}$.

Solution. Multiply both the numerator and denominator by $2+3 i$,

thus

$$
\frac{(5-3 i)(2+3 i)}{(2-3 i)(2+3 i)}=\frac{10-6 i+15 i-9 i^{2}}{4-9 i^{2}}=\frac{19+9 i}{13} \text {. }
$$

\section{EXERCISE 3}

1. Factor the following expressions:
(a) $x^{2}-2$,
(b) $x^{2}-7$,
(c) $x^{3}-3$
(d) $x^{3}+3$
(e) $x^{2}+1$,

(f) $x^{2}+4$, (g) $2 x^{2}-3$, (h) $3 x^{3}-5$, (i) $4 x^{2}+9$, (j) $2 x^{3}+5$. Ans. $(a)(x-\sqrt{ } 2)(x+\sqrt{ } 2)$. 


\section{A SHORT COURSE IN MATHEMATICS}

2. Simplify the following expressions:
(a) $\sqrt{ } 15 \times \sqrt{ } 18$,
(b) $(2 \sqrt{ } 8+\sqrt{ } 72)(5 \sqrt{ } 3+2 \sqrt{ } 96)$,
(c) $\sqrt{3+\sqrt{ } 5} \times \sqrt{3-\sqrt{ } 5}$.

3. Reduce to simplest form :
(a) $\left(x^{\frac{1}{3}} y^{\frac{2}{3}}\right)^{\frac{1}{2}} \div\left(y^{-\frac{1}{2}} y^{2}\right)^{\frac{1}{2}}$.
(b) $\frac{3^{x-1}\left(3^{-1}\right)^{x}}{\left(3^{x}\right)^{-1}\left(3^{x-1}\right)}$ Ans. (b) 1.

4. Reduce to its simplest form :

$$
\frac{\sqrt{ } a+\sqrt{ } b}{\sqrt{ } a-\sqrt{ } b} . \quad A n s . \quad \frac{a+\bar{b}+2 \sqrt{a b}}{a+b} .
$$

Suggestion. Multiply both terms by $\sqrt{ } a+\sqrt{ } b$.

Reduce also

$$
\frac{3 \sqrt{ } x+4 \sqrt{ } y}{2 \sqrt{ } x+\sqrt{ } y}
$$

5. Simplify each of the following expressions:
(a) $(3+4 \sqrt{-1})^{2}$,
(b) $(3-2 \sqrt{-1})(3+2 \sqrt{-1})$,
(c) $\frac{5+\sqrt{-1}}{2+\sqrt{-1}}$ Ans. (c) $\frac{11-3 i}{5}$.

6. Find the following products :
(a) $(x+i)(x-i)$.
(b) $(x+\sqrt{ } 3)(x-\sqrt{ } 3)$.
(c) $(y+i \sqrt{ } 2)(y-i \sqrt{ } 2)$. Ans. (c) $y^{2}+4$. 


\section{LESSON IV. - SOLUTION OF QUADRATIC EQUATIONS}

4.1. Quadratic Equations. A quadratic equation in $x$ is an equation in which $x^{2}$ is the highest power of $x$ which occurs in the equation after it has been reduced to its simplest form. If $x$ occurs only in the first power, the equation is said to be a simple, or linear, equation in $x$. If the highest power of $x$ which occurs in the equation is $x^{3}$, the equation is called a cubic equation in $x$. If $x^{4}$ is the highest power of $x$ which occurs the equation is called quartic. Frequently equations are referred to as being of the first, or second, or third, etc., degree in $x$ according as the highest power of $x$ which occurs in the equation is the first power, the second, third, etc.

Illustration. $3 x=4$ and $x-5=0$ are linear or first degree equations; $x^{2}=3, x^{2}+3 x=1$, and $2 x^{2}-x+12=0$ are quadratic or second degree equations; $x^{3}-1=0,3 x^{3}+4 x^{2}-5 x+6=0, x^{3}=x^{2}+4$ are cubic or third degree equations.

4.2. Roots of an Equation. To solve an equation is to find the value or values of the unknown quantity for which the equation is true; these values are said to satisfy the equation and are called the roots of the equation. The purpose of this lesson is to find the roots of any quadratic equation in one unknown.

Illustration. The equation $x^{2}-7 x+12=0$ is satisfied by each of the values $x=3$ and $x=4$, for substituting 4 for $x$ we have $4^{2}-7 \times 4$ $+12=16-28+12=0$, and substituting 3 for $x$ we have $3^{2}-7 \times 3+12$ $=9-21+12=0$. The numbers 4 and 3 are therefore the roots of the equation $x^{2}-7 x+12=0$. To solve the equation is to find these numbers. 
4.3. Solution of Equations. Consider the product of two or more factors such as $P=a \times b \times c$. Certainly if $a$ and $b$ and $c$ are each different from zero, the product $P$ must be different from zero, for we can never obtain zero by multiplying one number by another no matter how small one or both of the numbers may be. On the other hand, if one of the factors $a$ or $b$ or $c$ is zero, $P$ will be zero no matter what the other factors are, for if zero is taken ever so many times the result is still zero. We may state this important conclusion as follows :

An expression is zero if, and only if, it has a factor which is zero.

Now every equation may be written with all its terms transposed to the left hand side of the equality sign leaving zero on the right hand side of the equality sign. We thus have an expression equal to zero. But we have just seen that this is possible only if some one of its factors is zero. We thus see that the problem of solving an equation resolves itself into the problem of factoring the expression which results from transposing all the terms of the equation to one side of the equality sign.

Illustrations. Consider the equation used in the preceding illustration, namely the equation $x^{2}-7 x+12=0 . x^{2}-7 x+12$ is zero if, and only if, one of its factors is equal to zero. The factors of $x^{2}-7 x+12$ are $x-4$ and $x-3$, hence $x^{2}-7 x+12=0$ only if either $x-4=0$ or if $x-3=0$. Now $x-4=0$ if $x=4$, and $x-3=0$ if $x=3$; the equation therefore is satisfied by the two numbers 4 and 3 and by no other numbers.

Again, let it be required to solve the equation $x^{3}=3 x^{2}-2 x$. Transposing all the terms to the left hand side of the equation we have $x^{3}-3 x^{2}$ $+2 x=0$. The factors of $x^{3}-3 x^{2}+2 x$ are $x, x-1$, and $x-2$, therefore $x^{3}-3 x^{2}+2 x=0$ only if $x=0$, or if $x-1=0$, or if $x-2=0$. If $x-1=0, x=1$; if $x-2=0, x=2$; the roots of the equation $x^{3}=3 x^{2}$ $-2 x$ are therefore 0,1 , and 2 . 
4.4. Equivalent Equations. Equations having the same roots are said to be equivalent. $x=1$, and $x-1=0$ are equivalent equations, so are the two equations $x^{3}=3 x^{2}-2 x$ and $x^{3}-3 x^{2}+2 x=0$ used in the illustration above. But the two equations $x^{3}=3 x^{2}-2 x$ and $x^{2}=3 x-2$, the second being obtained from the first by dividing by $x$, are not equivalent, for the first equation has the three roots $0,1,2$ while the second has only the two roots 1 and 2 .

The operations which result in equivalent equations are :

(a) Adding or subtracting the same quantity to or from both members of an equation (transposition of terms).

(b) Multiplying or dividing both members of an equation by any quantity which does not involve $x$ (clearing of fractions and dividing out common factors).

When we subject an equation to any other operation, as when we square both members of an equation, or take the square-root of both sides of an equation, or when we multiply or divide both members of an equation by some factor involving $x$, the resulting equation is no longer necessarily equivalent to the original equation. The roots found from the resulting equation may or may not satisfy the original equation. The roots so found must be tested by substituting them for $x$ in the original equation. Those that satisfy the equation are its roots, the others are not.

Illustration. Find the value of $x$ in the equation

$$
\sqrt{2 x+9}-\sqrt{x-4}=\sqrt{x+1} \text {. }
$$

Solution. Squaring both sides of the equation we obtain

$$
(2 x+9)-2 \sqrt{2 x+9} \sqrt{x-4}+(x-4)=x+1 .
$$

Transposing and collecting terms, $2 x+4=2 \sqrt{2 x+9} \sqrt{x-4}$.

Dividing by 2 ,

$$
x+2=\sqrt{2 x+9} \sqrt{x-4} .
$$

Squaring both members again, $\quad(x+2)^{2}=(2 x+9)(x-4)$.

Multiplying out, $x^{2}+4 x+4=2 x^{2}+x-36$.

Transposing,

$$
x^{2}-3 x-40=0 \text {. }
$$


Factoring, $\quad(x-8)(x+5)=0$, from which $x=8$, or $x=-5$.

In solving this equation we squared the equation twice, hence we can not be sure that the numbers 8 and -5 satisfy the original equation. Substituting the value 8 for $x$ in the original equation the equation is satisfied, if we substitute the value -5 it is not. The given equation has therefore only one root, namely 8.

\subsection{General Form of the Quadratic Equation. Every} quadratic equation when properly simplified may be written in the form $a x^{2}+b x+c=0$, where $a$ represents the coefficient of $x^{2}, b$ the coefficient of $x$, and $c$ the term which is free from $x$. $\quad b$ and $c$ may have any given values including zero, but $a$ cannot be zero, for if it were the equation would no longer be a quadratic equation. It would then be a simple equation, which we are not considering at this time. Since $a$ cannot be zero we may divide the equation by $a$ and obtain the equivalent equation $x^{2}+\frac{b}{a} x+\frac{c}{a}=0$, which if we write $p$ for $\frac{a}{b}$ and $q$ for $\frac{c}{a}$ assumes the still simpler form $x^{2}+p x+q=0$.

Example. Consider the quarlratic equation $4 x^{2}-5=3 x$. Transposing we have the equivalent equation $4 x^{2}-3 x-5=0$, which is of the form $a x^{2}+b x+c=0, a$ being $4, b,-3$ and $c,-5$. If we furthermore divide the equation by the coefficient of $x^{2}$, namely 4 , we obtain the equivalent equation $x^{2}-\frac{3}{4} x-\frac{5}{4}=0$, which is of the form $x^{2}+p x+q=$ 0 , where $p=-\frac{3}{4}$ and $q=-\frac{5}{4}$. Again $x^{2}+x=0$ is of the form $x^{2}+p x$ $+q=0$, where $p=1$ and $q=0 . x^{2}-5=0$ is of the form $x^{2}+p x+q$ $=0$, where $p=0$ and $q=-5$.

\subsection{The General Solution of the Quadratic Equation.} To solve the equation $x^{2}+p x+q=0$ we must first factor the trinomial $x^{2}+p x+q$. If the roots are rational numbers this can generally be done by inspection as in 1.9 (c). If the factors cannot be found by inspection we proceed as follows. Let us represent the unknown factors by $x-r^{\prime}$ and $x-r^{\prime \prime}$; then $\left(x-r^{\prime}\right)\left(x-r^{\prime \prime}\right)=x^{2}-\left(r^{\prime}+r^{\prime \prime}\right) x+r^{\prime} r^{\prime \prime}=x^{2}+p x+q$, and 
hence by comparing the coefficients we see that $r^{\prime}+r^{\prime \prime}=-p$, and $r^{\prime} r^{\prime \prime}=q$. We can now easily find $r^{\prime}$ and $r^{\prime \prime}$, for since

$$
\begin{aligned}
r^{\prime}+r^{\prime \prime} & =-p \\
\left(r^{\prime}+r^{\prime \prime}\right)^{2} & =r^{\prime 2}+2 r^{\prime} r^{\prime \prime}+r^{\prime \prime 2}=p^{2}, \\
4 r^{\prime} r^{\prime \prime} & =4 q .
\end{aligned}
$$

Subtracting $\quad r^{\prime 2}-2 r^{\prime} r^{\prime \prime}+r^{\prime \prime 2}=p^{2}-4 q$.

Taking the square-root $r^{\prime}-r^{\prime \prime}=\sqrt{p^{2}-4 q}$.

But

hence

$$
\begin{aligned}
& r^{\prime}+r^{\prime \prime}=-p, \\
& r^{\prime}=\frac{\left(r^{\prime}+r^{\prime \prime}\right)+\left(r^{\prime}-r^{\prime \prime}\right)}{2}=\frac{-p+\sqrt{p^{2}-4 q}}{2}, \\
& r^{\prime \prime}=\frac{\left(r^{\prime}+r^{\prime \prime}\right)-\left(r^{\prime}-r^{\prime \prime}\right)}{2}=\frac{-p-\sqrt{p^{2}-4 q}}{2} .
\end{aligned}
$$

To find the roots of the equation, it only remains to put each factor equal to zero and solve for $x$. From $x-r^{\prime}=0$ we find $x=r^{\prime}$, from $x=r^{\prime \prime}=0$ we find $x=r^{\prime \prime}$, the roots of the equation $x^{2}+p x+p=0$, are therefore

$$
r^{\prime}=\frac{-p+\sqrt{p^{2}-4 q}}{2}, \quad r^{\prime \prime}=\frac{-p-\sqrt{p^{2}-4 q}}{2} .
$$

Examples. (a) Solve the equation $x^{2}-7 x+12=0$. Here $p=-7$, $q=12$. Substituting in the formulas $r^{\prime}$ and $r^{\prime \prime}$ we find

$$
r^{\prime}=\frac{7+\sqrt{49-48}}{2}=\frac{7+1}{2}=4, r^{\prime \prime}=\frac{7-\sqrt{49-4 \varepsilon}}{2}=\frac{7-1}{2}=3 .
$$

(b) Solve the equation $2 x^{2}-3 x-7=0$. Here $p=-\frac{3}{2}, q=-\frac{7}{2}$.

Hence $r^{\prime}=\frac{+\frac{3}{2}+\sqrt{\frac{9}{4}+14}}{2}=\frac{3+\sqrt{ } 65}{4}=\frac{3+8.062257}{4}=\frac{11.062257}{4}$ $=2.765564$,

$$
\begin{aligned}
r^{\prime \prime}=\frac{+\frac{3}{2}-\sqrt{\frac{9}{4}+14}}{2}=\frac{3-\sqrt{ } 65}{4} & =\frac{3-8.062257}{4}=\frac{-5.062257}{4} \\
& =-1.265564 .
\end{aligned}
$$

4.7. Second Method of Solving the Quadratic Equation. Completing the Square. If we add and subtract $p^{2} / 4$ the trinomial $x^{2}+p x+q$ takes the equivalent form

$$
x^{2}+p x+\frac{p^{2}}{4}-\left(\frac{p^{2}}{4}-q\right) \text {. }
$$


Now $x^{2}+p x+\frac{p^{2}}{4}$ is a perfect square, namely $\left(x+\frac{p}{2}\right)^{2}$ and $\frac{p^{2}}{4}-q$ being a known number is a square, namely the square of its own square-root. The trinomial therefore takes the form $\left(x+\frac{p}{2}\right)^{2}-\left(\sqrt{\frac{p^{2}}{4}-q}\right)^{2}$, which is the difference of two squares and may be factored as shown in $1.7(d)$. The factors are $\left(x+\frac{p}{2}\right)+\sqrt{\frac{p^{2}}{4}-q}$ and $\left(x+\frac{p}{2}\right)-\sqrt{\frac{p^{2}}{4}-q}$; each of these factors equated to zero gives a root of the quadratic equation $x^{2}+p x+q=0$.

Examples. (a) Solve the equation $x^{2}-7 x+12=0$. Carrying out the steps indicated above, we have in turn

$$
\begin{aligned}
x^{2}-7 x+12 & =x^{2}-7 x+\frac{49}{4}-\left(\frac{49}{4}-12\right)=\left(x-\frac{7}{2}\right)^{2}-\frac{1}{4} \\
& =\left(x-\frac{7}{2}+\frac{1}{2}\right)\left(x-\frac{7}{2}-\frac{1}{2}\right)=(x-3)(x-4)=0 .
\end{aligned}
$$

Putting $x-3=0$ we find $x=3$, putting $x-4=0$ we find $x=4$.

(b) Solve the equation $2 x^{2}-3 x-7=0$. Dividing by 2 we obtain the equivalent equation

$$
\begin{aligned}
x^{2}-\frac{3}{2} x-\frac{7}{2} & =0 . \quad \text { Adding and subtracting }\left(\frac{3}{4}\right)^{2} \text { we obtain } \\
x^{2}-\frac{3}{2} x-\frac{7}{2} & =x^{2}-\frac{3}{2} x+\frac{9}{16}-\left(\frac{9}{16}+\frac{7}{2}\right)=x^{2}-\frac{3}{2} x+\frac{9}{16}-\frac{65}{16} \\
& =\left(x-\frac{3}{4}\right)^{2}-\left(\sqrt{\frac{65}{16}}\right)^{2}=(x-.75)^{2}-(2.015564)^{2} \\
& =(x-.75+2.015564)(x-.75-2.015564) \\
& =(x+1.265564)(x-2.765564)=0 .
\end{aligned}
$$

The roots of the equation $2 x^{2}-3 x-7=0$ are therefore

$$
x=-1.265564 \text {, and } x=+2.765564 \text {. }
$$

\subsection{Roots of the Quadratic in Terms of the Coefficients} $a, b$, and $c$. If the equation is given in the form $a x^{2}+b x+c$ $=0$ it is not necessary to divide through by the coefficient $a$ before solving it. For since $p=\frac{b}{a}$ and $q=\frac{c}{a}$, we may substitute these values for $p$ and $q$ in the expressions $\frac{-p+\sqrt{p^{2}-4 q}}{2}$ and $\frac{-p-\sqrt{p^{2}-4 q}}{2}$ of Art. 4.6 and obtain 
after a slight reduction,

$$
r^{\prime}=\frac{-b+\sqrt{b^{2}-4 a c}}{2 a}, \quad r^{\prime \prime}=\frac{-b-\sqrt{b^{2}-4 a c}}{2 a}
$$

for the roots of the equation $a x^{2}+b x+c=0$.

Example. Solve the equation $2 x^{2}-3 x-7=0$. Here $a=2, b=-3$, $c=-7$, hence the roots are

$$
r^{\prime}=\frac{3+\sqrt{9+56}}{4}=\frac{3+\sqrt{65}}{4}=2.765564, \quad r^{\prime \prime}=\frac{3-\sqrt{65}}{4}=-1.265564 .
$$

4.9. Suggestion to the Student. The roots of any quadratic equation may be found from the formulas of 4.6 by substituting for $p$ and $q$ their values, or from the formulas of 4.8 by substituting for $a, b$, and $c$ their values. This is the most expeditious method. The student should, however, understand his formulas as well as be able to use them, and for this reason it is recommended that he use the method explained in 4.7.

\section{EXERCISE 4}

1. Find all the roots of each of the following equations :
(a) $(x-1)(x-5)=0$,
(b) $x(x-2)(x+3)=0$,
(c) $2 x^{2}(x+1)(2 x-1)=0$.

2. Solve the following equations :
(a) $x^{2}-1=0$,
(b) $x^{2}-3=0$,
(c) $4 x^{2}-9=0$,
(d) $x^{2}+1=0$,
(e) $9 x^{2}+4=0$,
(f) $x\left(x^{2}-4\right)\left(x^{2}-2\right)\left(x^{2}+5\right)=0$,
(g) $x^{4}-1=0$.

3. Solve by inspection (factoring at sight) the equations :
(a) $x^{2}-3 x-4=0$,
(b) $y^{2}+12 y+32=0$,
(c) $t^{3}-t^{2}-2 t=0$.
Ans. (c) 0, 2, - 1 .

4. Solve by completing the square:
(a) $x^{2}+5 x+1=0$,
(b) $y^{2}-2 y-2.2=0$,
(c) $2 k^{2}+3.4 k-1.2=0$.
Ans. 0.3, - 2 . 
5. Solve for $x$ :
(a) $\frac{x+11}{x+3}=\frac{2 x+1}{x+5}$,
(b) $x-1=\sqrt{x-1}$
(c) $x+\sqrt{ } x=20$,
(d) $\sqrt{1+4 x}-\sqrt{1-4 x}=4 \sqrt{x}$,
Ans. (b) 1, 2.
(e) $\frac{x}{a}+\frac{a}{x}=\frac{a}{b}+\frac{b}{a}$.
Ans. (d) 0 .

6. Form an equation whose roots are:
(a) 2,3 ;
(b) $0,2,-1$;
(c) $\sqrt{ } 3,-\sqrt{ } 3$;
(d) $1,-1, \sqrt{ } 2,-\sqrt{ } 2$;
(e) $2+\sqrt{ } 3,2-\sqrt{ } 3$;
(f) $3 i,-3 i$; (g) $2+i, 2-i$;
(h) $1+i, 1-i, \sqrt{ } 2,-\sqrt{ } 2$.

Ans. (d) $x^{4}-3 x^{2}+2=0,(f) x^{2}+9=0$, (h) $x^{4}-2 x^{3}+4 x$ $-4=0$. 
LESSON V. - APPLIED PROBLEMS INVOLVING QUADRATIC EQUATIONS

5.1. Character of the Roots of a Quadratic. We have seen in (4.8) that the quadratic equation $a x^{2}+b x+c=0$ has two roots

$$
x=\frac{-b \pm \sqrt{b^{2}-4 a c}}{2 a} .
$$

From this it appears that.

(a) When $b^{2}-4 a c=0$ the two roots are equal;

(b) When $b^{2}-4 a c>0$ both roots are real and distinct;

(c) When $b^{2}-4 a c<0$ both roots are imaginary and distinct;

(d) When $b^{2}-4 a c$ is a perfect square, both roots are rational;

(e) When $c=0$ one root is zero and the other is $-b / a$;

$(f)$ When $b=0$ the roots are numerically equal but opposite in sign ;

(g) When $b=0$ and $c=0$ both roots are equal to zero.

5.2. Interpretation of Roots. While every quadratic equation has two roots, a problem which leads to a quadratic equation, that is, a problem whose solution depends upon solving some quadratic equation, may have two, or only one, or even no solution at all, for while the equation upon which the solution depends has two roots, one root only or neither of the roots may admit of interpretation. No matter what the character of the roots, integral or fractional, positive or negative, rational or irrational, real or imaginary, whether they admit 
or do not admit of interpretation depends upon the problem which gives rise to the equation and upon nothing else. All this will be much plainer by considering some examples.

\subsection{Problems Admitting Two Solutions.}

Example. The Emergency Fleet Corporation awarded two contracts for the construction of merchant vessels, one for $\$ 2,700,000$, the other for $\$ 3,080,000$. The second contract called for one more vessel than the first and the cost per vessel under the second contract exceeded by $\$ 10,000$ the cost per vessel in the first contract. How many vessels did each contract call for?

Solution. Let $x=$ the number of vessels in the first contract, then $x+1=$ the number of vessels in the second contract, $\$ 2,700,000 / x=$ the cost per vessel under the first contract, and $\$ 3,080,000 /(x+1)$ the cost per vessel under the second contract. The difference between the two is $\$ 10,000$. We have therefore the equation

$$
\frac{3080000}{x+1}-\frac{2700000}{x}=10000 \text {. }
$$

Clearing of fractions,

$$
3080000 x-2700000(x+1)=10000 x(x+1) .
$$

Multiplying out and dividing by 10000 ,

$$
308 x-270 x-270=x^{2}+x .
$$

Transposing and collecting terms,

$$
\begin{aligned}
& x^{2}-37 x+270=0 . \\
& (x-10)(x-37)=0 \text {, } \\
& x=10, \text { or } x=27 \text {. }
\end{aligned}
$$

In this example both roots admit of interpretation. The first contract called for either 10 vessels or 27 vessels. If there were 10 vessels the cost per vessel under the first contract was $\$ 2,700,000 / 10$, or $\$ 270,000$, and the cost per vessel under the second contract was $\$ 3,080,000 / 11$, or $\$ 280,000$, and the difference in these costs is $\$ 10,000$. On the other hand if the number of vessels in the first contract was 27 , the cost per vessel was $\$ 2,700,000 / 27$, or $\$ 100,000$, the cost per vessel under the second contract was $\$ 3,080,000 / 28$, or $\$ 110,000$, and the difference between these costs is again $\$ 10,000$. There is no way of telling which one of these answers is the answer unless we have some further information as, for instance, the size of the vessels. 


\subsection{Problems with Two Solutions Which Are Only Appar- ently Different.}

Example. A wharf $125 \mathrm{ft}$. long consists of two square platforms joined together. The total wharf area is $8125 \mathrm{sq}$. ft. Find the dimensions of the two squares.

Solution. Denote the length of the side of one square by $x$, then the length of the side of the second square must be $125-x$, and the sum of their areas is $8125 \mathrm{sq}$. $\mathrm{ft}$. This gives us the equation

$$
x^{2}+(125-x)^{2}=8125 .
$$

Multiplying out, $\quad x^{2}+15625-250 x+x^{2}=8125$.

Transposing and collecting, $2 x^{2}-250 x+7500=0$.

Dividing by 2 ,

$$
x^{2}-125 x+3750=0 \text {. }
$$

Factoring,

hence the two roots are

$$
\begin{aligned}
(x-50)(x-75) & =0, \\
x=50, & \text { and } x=75 .
\end{aligned}
$$

The length of one side of the first platform is therefore either $50 \mathrm{ft}$. or $75 \mathrm{ft}$. If it is $50 \mathrm{ft}$. the dimension of the other square is $75 \mathrm{ft}$., if it is $75 \mathrm{ft}$. then the dimension of the second square is $50 \mathrm{ft}$. Whichever root we choose the conclusion is the same, the wharf consists of two squares whose sides measure $50 \mathrm{ft}$. and $75 \mathrm{ft}$.

\subsection{Problems Which Admit of Only One Solution.}

Example. A printed page 5 in. $\times 7$ in. has a margin of uniform width occupying half the area of the page. Find the width of the margin.

Solution. Let $x$ stand for the width of the margin in inches, then $5-2 x$ and $7-2 x$ represent the dimensions of the printed part of the page which is equal to one half of the area of the page. We have therefore

$$
(5-2 x)(7-2 x)=5 \times 7 \div 2=17.5 \text {. }
$$

Multiplying out,

$$
35-24 x+4 x^{2}=17.5 \text {. }
$$

Transposing and collecting,

$$
4 x^{2}-24 x+17.5=0 \text {. }
$$

Dividing by 4 ,

$$
x^{2}-6 x+4.375=0 \text {. }
$$

Completing the square, $x^{2}-6 x+9-(9-4.375)=0$, or

$$
(x-3)^{2}-(2.15058)^{2}=0 \text {. }
$$

Factoring,

$$
(x-3+2.15058)(x-3-2.15058)=0 \text {, }
$$
or

$$
(x-0.84942)(x-5.15058)=0 \text {, }
$$

from which

$$
x=0.84942 \text {, or } x=5.15058 \text {. }
$$


The first root gives us an answer to the problem, a margin of 0.85 of an inch on a page $5 \mathrm{in}$. by $7 \mathrm{in}$. will take up approximately one half of the page. The second root $x=5.15$ does not admit of interpretation, for clearly a page 5 in. wide can not have a margin of more than 5 in.

\subsection{Problems Which Admit of No Solution.}

Example. A brigade of 7000 men is to be arranged in the form of a hollow square with 100 men on each side. How many ranks deep will the men have to stand?

Solution. Let $x$ represent the number of ranks on each side of the square. Then the dimension of the hollow part of the square will be $100-2 x$. If the square were completely filled it would contain $100^{2}$ men, but since it requires $(100-2 x)^{2}$ men to fill the hollow part of the square, the hollow square requires $100^{2}-(100-2 x)^{2}$ men, which must equal 7000. This gives us the equation

$$
100^{2}-(100-2 x)^{2}=7000 .
$$

- Transposing, $(100-2 x)^{2}-100^{2}+7000=0$, from which

$$
(100-2 x)^{2}-3000=0 \text {. }
$$

Factoring, $(100-2 x+\sqrt{3000})(100-2 x-\sqrt{3000})=0$.

Solving,

$$
x=\frac{100 \pm \sqrt{3000}}{2}=\frac{100 \pm 54.772}{2},
$$

from which

$$
x=77.386 \text {, or } x=22.614 \text {. }
$$

Now neither of these roots admits of interpretation, for manifestly the number of ranks must be a whole number. The conclusion is that it is impossible to arrange 7000 men in a hollow square with 100 men on a side.

5.7. Imaginary Roots. It is sometimes erroneously stated that when the roots of an equation are imaginary the problem which gave rise to the equation is impossible. It is true that imaginary roots admit of interpretation less frequently than do real roots. We have seen that even positive roots do not always admit of interpretation; we should therefore expect to find many cases in which negative roots and more especially imaginary roots indicate some impossible condition. If the unknown quantity represents a number of men and the answer 
comes out fractional, it indicates that the problem is impossible, for the number of men must be an integer. If the unknown represents an area or a volume, a negative result ordinarily indicates some absurd requirement, but if the unknown represents a resource, a negative result indicates a liability; if the unknown represents distance traveled in one direction, a negative result indicates distance traveled in the opposite direction, and so on. Similarly we may interpret imaginary results, provided the unknown represents a quantity which admits of imaginary interpretation. This is the case wherever we are dealing with magnitudes which have direction, such as forces, velocities, and accelerations, for such magnitudes may be represented by imaginary numbers. In the study of theoretical physics, especially in the study of electricity and magnetism, a thorough knowledge of imaginary and complex numbers is indispensable.

\section{EXERCISE 5}

Solve the following problems, being careful to consider all the solutions, and to interpret all those which admit of interpretation.

1. A field $170 \mathrm{rd}$. long is made square by cutting 6000 sq. rd. off one end. Find the width of the field. Ans. $50 \mathrm{rd}$.

2. A rectangular field containing 100 acres requires 520 rods of fencing to inclose it. Find its dimensions.

3. A detachment of troops was marching in close column with 15 men more in depth than in front. Before going into action the front was so extended as to add 840 men to each rank and to reduce the number of ranks to 5 . Find the number of men in the detachment.

Ans. 4500.

4. Two square lots within a common inclosure contain a combined area of 890,000 sq. ft. The wall inclosing them 
measures $4200 \mathrm{ft}$. Find the dimensions of the lots. Two sets of answers.

5. A city addition is laid out in square blocks, with streets $35 \mathrm{ft}$. wide. The streets occupy one fourth of the entire area. Find the size of the blocks. Ans. $261.24 \mathrm{ft}$.

6. A squadron came out of an engagement with 9 ships, having lost as many per cent of its ships as the original number of ships in the squadron. How many vessels were lost?

7. Can a brigade of 5000 men be drawn up in the form of a hollow square with 75 men on a side? How many ranks deep will the men have to stand? Ans. 25.

8. In order to save 2 hours in covering a distance of 18 miles a company increases its rate of march by $\frac{3}{4}$ mile per hour. What was its former rate of march?

9. The corners of a square the length of whose side is $200 \mathrm{ft}$. are cut off in such a way that a regular octagon remains. Find the length of one side of the octagon.

$$
\text { Ans. } 200(\sqrt{ } 2-1)=41.42 \mathrm{ft} \text {. }
$$

10. A debt of $\$ 5000$ is repaid by two equal annual installments of $\$ 2746.31$ each. Find the rate of interest.

11. $\$ 2000$ at compound interest amounts to $\$ 2226.05$ in two years. Find the rate of interest.

Ans. $5 \frac{1}{2} \%$.

12. What is the price of eggs per dozen so that one more egg for 42 cents amounts to a reduction of 12 cents per dozen?

13. A square box 5 in. high has $96 \mathrm{sq}$. in. more in its lateral area than in its bottom. Find the dimensions of the box. Ans. $8 \times 8 \times 5$, or $12 \times 12 \times 5$.

14. Two cubical blocks of stone one placed on top of the other have a combined height of $10 \mathrm{ft}$. Their combined volume is $280 \mathrm{cu}$. ft. Find the dimensions of each block. 
15. Two squads of men working together dig a section of trench in $5 \mathrm{hr}$. The squads working separately dig an equal section each, the first squad finishing $2 \mathrm{hr} .15 \mathrm{~min}$. before the second. What time did each squad make in digging its section?

Ans. 9 hr.; 11 hr. $15 \mathrm{~min}$.

16. A crew which is able to row $4.5 \mathrm{mi}$. per hour in still water rows $6 \mathrm{mi}$. up the Sound and back $4 \mathrm{mi}$. in $2 \mathrm{hr}$. $20 \mathrm{~min}$. How fast did the tide run and in what direction? (Two answers.)

17. A crew rows $6 \frac{1}{2} \mathrm{mi}$. downstream and back again in $3 \mathrm{hr}$. $28 \mathrm{~min}$. If the current of the stream is $2 \frac{1}{2} \mathrm{mi}$. per hour what is the rate of the crew in still water?

18. The output of a certain plant is to be taxed so as to yield a revenue of $\$ 20,000$. The normal output is $\$ 1,000,000$, but it is anticipated that the effect of levying the tax will be to decrease the output by a per cent equal to the tax rate. Assuming this to be true, at what rate must the tax be fixed so as to yield the desired revenue?

19. Two lights; $A, B$, of 100 and 64 candle power respectively, are $54 \mathrm{ft}$. apart. Assuming that the intensity of light varies inversely as the square of the distance of the source of light, find the point on the line joining $A$ and $B$ which is equally illuminated by each of the two lights.

Ans. $30 \mathrm{ft}$. or $270 \mathrm{ft}$. from $A$ toward $B$.

20. Two magnets whose strengths are in the ratio of 4 to 9 are placed at a distance of 20 inches from each other. Find a point on the line joining their centers at which a needle would be equally attracted by each. Note. - The intensity of magnetic attraction varies inversely as the square of the distance from the magnet.

21. If a body is thrown vertically upward its height at any time is given by the formula $s=v t-16.1 t^{2}$, when $v$ is the 
initial velocity of the body in feet per second, $t$ the time in seconds, and $s$ the height in feet. (a) If $v=100$, how long will it take the body to rise $100 \mathrm{ft}$.? (b) How long before its returns to the earth? (c) What is the greatest height it attains?

Ans. (a) 1.25 seconds or 4.96 seconds.

(b) 6.21 seconds.

(c) 155.25 feet.

22. The side of a regular decagon inscribed in a circle is found as follows: Divide the radius of the circle into two segments so that the whole radius is to the larger segment as the larger segment is to the smaller segment. The larger segment is equal in length to the side of the required decagon. Find the length of a side of a decagon inscribed in a circle whose radius is $10 \mathrm{ft}$. Ans. $x=5(\sqrt{ } 5-1)=6.18 \mathrm{ft}$. 


\section{LESSON VI - SOME PROBLEMS IN GUNNERY}

6.1. Definitions. The path, GPT, of a projectile from the time it leaves the gun till it hits the ground or target is called its trajectory.

The distance, $G T$, from the gun to the target is called the range of the gun.

The straight line, $G T$, connecting the muzzle of the gun and the target is called the line of sight.

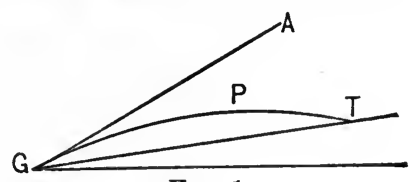

FIG. 1

$G A$, the direction of the projectile the moment it leaves the gun, is called the line of departure.

The angle, $A G T$, which the line of departure makes with the line of sight is called the angle of departure.

6.2. Units of Measure. The yard is the usual unit of distance employed by the United States Field Artillery.

The unit of angular measure is the mil, which is approximately the $\frac{1}{1571}$ part of a right angle; for practical reasons the $\frac{1}{1600}$ part is used in computations, which gives a result nearly $2 \%$ too small.

On the assumption that $1 \mathrm{mil}$ is approximately $\frac{1}{1600}$ part of a right angle, it follows that $1600 \mathrm{mils}=90 \times 60^{\prime}=5400^{\prime}$, or $1^{\prime}=\frac{1600}{5400} \mathrm{mils}=.3 \mathrm{mils}$ (nearly), hence we have the following approximate

Conversion Rules, (a) To change from minutes of arc to mils multiply by $\frac{3}{10}$.

(b) To change from mils to minutes of arc multiply by $\frac{10}{3}$ or $3 \frac{1}{3}$. 
6.3. Departure and Range. The departure corresponding to any given range, or vice versa, can be computed to any desired degree of accuracy, but the computations require considerable time. For this reason range tables have been constructed for the use of artillery officers in the field. In the absence of such tables approximation formulas are used which are sufficiently simple to be carried in the head and from which the departure corresponding to any given range, or the range corresponding to any given departure, may be quickly computed with an accuracy sufficient for most practical purposes.

For the standard 3-inch field gun used in the United States artillery service we have

$$
\phi=5 R(R+3),
$$

where $R$ is the range in thousands of yards, and $\phi$ is the angle of departure expressed in mils.

For the Vickers-Maxim gun, using a $12 \frac{1}{2}$-pound projectile, muzzle velocity 920 feet per second, we have

$$
\phi=8 R(R+6) \text {. }
$$

Examples. (a) Find the angle of departure corresponding to a range of 1000 yards, for a 3 -inch field gun.

Solution. In the above formula substitute $R=1$, then $\phi$ (in mils) $=5 \times 1(1+3)=20$.

(b) Find the range corresponding to an angle of departure of 50 mils.

Solution. In the above formula substitute $\phi=50$ and solve for $R$, thus,

or

$$
\begin{aligned}
5 R(R+3) & =5 R^{2}+15 R=50, \\
R^{2}+3 R-10 & =0 .
\end{aligned}
$$

Completing the square, $R^{2}+3 R+\frac{9}{4}-\left(\frac{9}{4}+10\right)=0$, or

$$
R^{2}+3 R+\frac{9}{4}-\left(\frac{49}{4}\right)=0 \text {, }
$$

that is,

$$
\left(R+\frac{3}{2}\right)^{2}-\left(\frac{7}{2}\right)^{2}=0 .
$$

Factoring, from which

$$
\begin{aligned}
\left(R+\frac{3}{2}+\frac{7}{2}\right)\left(R+\frac{3}{2}-\frac{7}{2}\right) & =0, \\
R=-5, \text { or } R & =2 .
\end{aligned}
$$

The root -5 does not admit of interpretation, the root $R=2$, since $R$ is expressed in thousands of yards, gives the range $2000 \mathrm{yd}$. 
6.4. The Time of Flight. The time it takes the projectile from a 3-inch field gun to reach the target is given with sufficient accuracy by the following simple formula:

$$
T=\frac{R}{10}(3 R+16)
$$

where $T$ is the time in seconds and $R$ the range in thousands of yards.

For the Vickers-Maxim 2.95-inch mountain gun

$$
T=\frac{2 R}{5}(R+6) \text {. }
$$

From such formulas the range can be determined by noting the time it takes for the projectile to strike the ground or target.

Example. In firing on an enemy position 1 mile away with a battery of Vickers-Maxim guns, the time of the first rounds fired was observed to be $5 \frac{3}{4}$ seconds. Was the range too strong or too short and by how much ?

Solution. Substituting $T=5.75$, the equation for $R$ is

or

$$
\frac{2}{5} R(R+6)=5.75
$$

Completing the square,

$$
R^{2}+6 R-14.375=0 .
$$

$$
R^{2}+6 R+9-(9+14.375)=0
$$

or $\quad(R+3)^{2}-(4.835)^{2}=0$.

Factoring, $(R+3+4.835)(R+3-4.835)=0$, from which

$$
R=-7.835 \text {, or } R=1.835 \text {. }
$$

The first root does not admit of interpretation, the second gives the range equal to $1835 \mathrm{yd}$. Since the enemy position is $1 \mathrm{mi}$., or $1760 \mathrm{yd}$., the range must be shortened by $75 \mathrm{yd}$.

6.5. Firing over a Mask. In masking a gun, that is, placing it in some concealed position, care must be taken to so locate the gun that the trajectory of the projectile clears the mask. If the height of the mask is known, the distance which a 3-inch gun must be placed behind the mask may be safely approximated by Percin's formula, which is

$$
4 h=x(10 R-x) \text {, }
$$


where $h$ is the height of the mask in yards, $R$ is the range in thousands of yards, and $x$ is the distance from the gun to the mask in hundreds of yards.

Example. An enemy position $3000 \mathrm{yd}$. away is to be attacked by a battery of 3-inch guns which are to be concealed by a mask $15 \mathrm{yd}$. high. Find at what distance behind the mask the guns must be placed.

Solution. We have given $h=15, R=3$, and we are to find $x$. Substituting in Percin's formula we have the quadratic equation

or

$$
\begin{aligned}
60 & =x(30-x), \\
x^{2}-30 x+60 & =0 .
\end{aligned}
$$

Completing the square,

$$
x^{2}-30 x+225-(225-60)=0,
$$

or $\quad(x-15)^{2}-(12.84)^{2}=0$.

Factoring, $(x-15+12.84)(x-15-12.84)=0$, from which

$$
x=2.16 \text {, or } x=27.84 \text {. }
$$

Since $x$ is in hundreds of yards, the first root gives us the answer to our question, namely, 216 yards.

The second root also admits of interpretation; a mask placed at a distance of 2784 yards from the guns, that is, 216 yards from the target, would also be cleared by the trajectory, but this fact is of no great importance to the gunner.

\section{EXERCISE 6}

1. Compute a range table for the following angles of departure $2^{\circ}, 2^{\circ} 30^{\prime}, 3^{\circ}, 3^{\circ} 30^{\prime}, 4^{\circ}, 4^{\circ} 30^{\prime}, 5^{\circ}, 10^{\circ}, 15^{\circ}$, for

(a) The 3-inch field gun.

Ans. 1574 yd., 1854 yd., 2112 yd., etc.

(b) The Vickers-Maxim 2.95-inch mountain gun. Ans. 674 yd., 824 yd., 969 yd., etc.

(Suggestion. Apply the formula in 4.6.)

2. When the time of flight is 10 seconds, find the range

(a) Of a 3-inch field gun;

(b) Of a Vickers-Maxim 2.95-inch mountain gun. Ans. (a) 3693 yd.; (b) 2831 yd. 
3. As in problem 2 , find the ranges when $T=15$ seconds; when $T=20$ seconds.

4. The range of a 3-inch field gun is 5000 yards, the height of the mask is 20 yards; at what distance behind the mask must the gun be located? Ans. $165.5 \mathrm{yd}$.

5. Solve problem 4 , when the range is 3760 yards and the mask is 25 yards high.

6. Will a 3 -inch field gun placed 150 yards behind a mask 60 feet high clear it when firing at a target 6000 yards distant? 


\section{LESSON VII - REVIEW}

1. What is meant by an integral expression in $x$ ? By a rational expression in $x$ ? Give illustrations of each. Write down an expression which is integral and rational in $x$. Rational, but fractional in $x$. Irrational in $x$.

2. Resolve into integral rational factors each of the following :

$$
\frac{x^{2}}{4}=9 ; \quad \frac{x^{2}}{4}-\frac{1}{3} a b+\frac{b^{2}}{9} ; \quad y^{2}+\frac{5}{6} y+\frac{1}{6} ; \quad 27 m^{3}-64 n^{3} .
$$

3. State and prove the Factor theorem. Show that $x-\frac{1}{2}$ and $x-\frac{1}{3}$ are factors of $6 x^{3}-41 x^{2}+31 x-6$. Find all the roots of the equation $6 x^{3}-41 x^{2}+31 x-6=0$.

4. State the fundamental laws of radicals. Illustrate each law by an example. State the three laws for exponents illustrating each law by an example.

5. What is the meaning of a fractional exponent? Of a negative exponent? Of a zero exponent? Find the value of $m^{\frac{3}{2}} l^{-\frac{1}{2}} t^{-1} p^{\frac{1}{2}}$ when $m=4, l=18, t=1, p=2$.

Simplify the expression $\left(x^{\frac{1}{2}}+y^{-\frac{1}{2}}\right)^{-3}$.

6. State the commutative law of addition, of multiplication. State the associative law of addition, of multiplication. What is meant by the distributive law? Show that this law is used in multiplying 1325 by 3 .

7. What are natural numbers? Show how each of the inverse processes - subtraction, division, root-extraction leads to numbers other than natural numbers. What is meant 
by an imaginary number? Give an example. Define complex number. Write down two unequal complex numbers and find $(a)$ their sum, (b) their difference, (c) their product, (d) their quotient. What is the square of $1+i$ ? The product of $1+i$ by $1-i$ ? Find two imaginary factors of the number 5 . Of $x^{2}+1$ ? Of $x^{2}+x+1$ ?

8. What is meant by a linear equation in $x$ ? By a quadratic equation in $x$ ? Give an example of each. What is the general form of a quadratic equation in $x$ containing the fewest number of coefficients?

9. What is meant by the roots of an equation? When are two equations said to be equivalent? What connection is there between the factors of an expression in $x$ and the roots of the equation obtained by putting the expression equal to zero? Illustrate your meaning by an example. Solve the equation $a x^{2}+b=0$, also $a x^{2}=0$, and $x^{2}+a x=0$. Solve the general equation $a x^{2}+b x+c=0$. If the equation is written in the form $x^{2}+p x+q=0$ what are its roots?

10. Discuss the character of the roots of the quadratic equation $a x^{2}+b x+c=0$. Without solving the equations following, state the character of the roots :

$$
\begin{aligned}
& 3 x^{2}+x-4=0, \quad 3 x^{2}+x+4=0, \quad x^{2}-\frac{1}{4} x+\frac{1}{4}=0, \\
& x^{2}-6 x+9=0 .
\end{aligned}
$$

Solve each of the foregoing equations by completing the square. 


\section{CHAPTER II}

\section{GRAPHIC METHODS}

\section{LESSON VIII - COÖRDINATES AND SIMPLE GRAPHS}

8.1. Longitude and Latitude. Every student is familiar with the method commonly employed in describing the position of a place on the earth's surface as, for instance, the position of a ship at sea. Two numbers are chosen, one giving the number of degrees between the meridian through the position of the ship and some arbitrarily agreed upon meridian as that of Greenwich, Paris, or Washington. This number is called the longitude of the ship. The second number is the number of degrees between the position of the ship and the equator. This is called the latitude of the ship. Two such numbers determine in reality four positions unless it be further specified whether the longitude is measured east or west from the principal meridian and the latitude north or south from the equator. Thus there are four different positions on the earth's surface whose longitude is $25^{\circ}$ and whose latitude is $15^{\circ}$, namely the positions, Long. $25^{\circ}$ E., Lat. $15^{\circ}$ $\mathrm{N}$.; Long. $25^{\circ}$ W., Lat. $15^{\circ} \mathrm{N}$.; Long. $25^{\circ}$ E., Lat. $15^{\circ} \mathrm{S}$; Long. $25^{\circ}$ W., Lat. $15^{\circ} \mathrm{S}$.

8.2. Government Land Surveys. A similar method for determining locations is employed in the United States Government land surveys. Every township included in the survey is designated by two numbers, one indicating its position with reference to some meridian (north and south line), the other giving its position with reference to some base line (east and west line). Every township in the first tier of townships east 
of the reference meridian is designated by range one east, contracted into R. 1 E., the townships in the second tier east by R. $2 \mathrm{E}$., those in the first tier west of the reference meridian by R. $1 \mathrm{~W}$., etc. Similarly every township in the first tier of townships north of the base line is referred to as township one north, contracted into T. 1 N., those in the second tier north as T. $2 \mathrm{~N}$., those in the first tier to the south of the base line as T. 1 S., etc. The position of any township in the region included in the survey may now be accurately described by two numbers, one indicating the range, the other the town to which it belongs. Thus the township described as R. 4 E., T. $3 \mathrm{~S}$., is the township common to the fourth range of townships east of the reference meridian and the third tier of townships south of the base line.

8.3. System of Coördinates. A system of lines, angles, or other quantities (such as longitude and latitude, ranges and towns) by means of which the position of a point or other element is determined in relation to certain fixed points, lines, or planes is called a system of coördinates. The quantities which determine any particular point or element are called the coördinates of that point or element.

8.4. Cartesian Coördinates. One of the simplest methods of describing the position of a point $P$ (fig. 2 ) in a plane is to measure the two distances $B P, A P$ of the point along lines drawn through $P$ parallel to the reference lines $X^{\prime} X$ and $Y^{\prime} Y$ respectively.

$B P$ (or the equal distance $O A$ ) is called the abscissa of the point $P$. This distance is usually denoted by $x$.

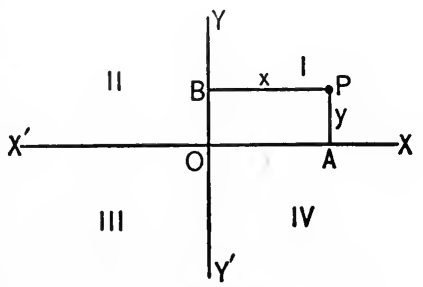

Fra. 2 
$A P$ (or the equal distance $O B$ ) is called the ordinate of the point $P$. This distance is usually denoted by $y$.

The abscissa and ordinate constitute the coördinates of the point $P$.

$X^{\prime} X$ (extending indefinitely in both directions) is known as the $x$-axis or axis of abscissas.

$Y^{\prime} Y$ (extending indefinitely in both directions) is known as the $y$-axis or axis of ordinates.

Taken together these two axes constitute the coördinate axes.

The point $O$ in which the coördinate axes intersect is known as the origin (of coördinates).

By the point $(x, y)$ we mean the point whose abscissa is $x$ and whose ordinate is $y$. Observe that the first number written is the abscissa and the second the ordinate, never the reverse.

The abscissa is considered positive $(+)$ if the point lies to the right of the $y$-axis, negative $(-)$ if to the left.

The ordinate is considered positive if the point lies above the $x$-axis, negative if below.

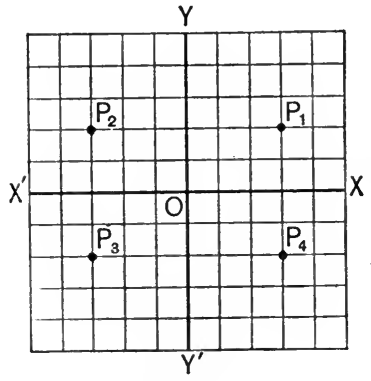

FrG. 3

Thus (fig. 3) the coördinates of the point $P_{1}$ are $(+3,+2)$, of $P_{2}(-3,+2)$, of $P_{3}(-3,-2)$, of $P_{4}(+3,-2)$. As in elementary algebra, when no sign is given the plus sign is understood.

The system of coördinates just described is known as the Cartesian system, after the French mathematician Descartes (1569-1650) who was the first to introduce the system.

The angle $X O Y$ (fig. 2) between the coördinate axes may be a right angle or an oblique angle; if a right angle the axes 
are said to be rectangular, if oblique the axes are said to be oblique. Unless the contrary is explicitly stated it is understood that the axes are rectangular.

8.5. The Four Quadrants. It is frequently convenient to refer separately to one or the other of the four regions into which the coördinate axes divide their plane. For this reason that part of the plane which lies to the right of the $y$-axis and above the $x$-axis is called the first quadrant, designated by $I$ in fig. 2. The other quadrants are numbered II, III, IV, as shown in the same figure.

8.6. Definition of Graph. We have seen that every point in a plane may be designated by two numbers, its coördinates. Conversely every pair of real numbers may be used to designate a point in a plane, namely the point which has the given numbers for coördinates. Suppose now that there are given two sets of numbers, let us say

$$
x_{1}, x_{2}, x_{3}, x_{4} \text {, etc. } \quad \text { and } y_{1}, y_{2}, y_{3}, y_{4} \text {, etc. }
$$

each number $y$ being in some way related to the corresponding number $x$, and that we wish to exhibit and study the relation between these two sets of numbers. A very simple and useful mode of procedure is the following:

We choose a pair of coördinate axes $X^{\prime} X$ and $Y^{\prime} Y$ (fig. 4) and plot (locate) the point $P_{1}$ which has $x_{1}$

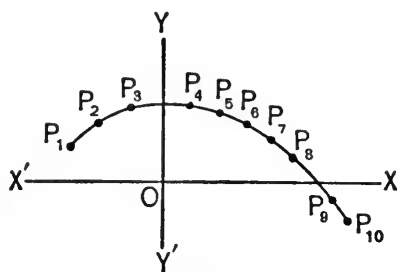

Fig. 4. for its abscissa and $y_{1}$ for its ordinate. Then we plot a second point $P_{2}$ using $x_{2}$ as abscissa and $y_{2}$ as ordinate. Similarly we plot each of the points $P_{3}=\left(x_{3}, y_{3}\right), P_{4}=\left(x_{4}, y_{4}\right)$, etc. The diagram obtained by commecting these points in succession by a 
smooth curve (as in fig. 4) or by a succession of straight lines (as in fig. 6) is called a graph.

In general a graph is a diagram which exhibits any sort of relation by means of systems of points and lines.

Whether the points, plotted as indicated above, should be connected by a smooth curve or by a succession of straight lines depends upon the relation between the two sets of numbers. If this relation is such that $y$ changes gradually with $x$ a smooth curve should be used, but if $x$ changes abruptly with $y$ it is better to use a broken line.

8.7. Smooth Curve Graphs. Temperature Graph. Let us consider the special case in which the $x$ 's represent the hours of the day and the $y$ 's the corresponding temperatures as recorded in the following table of observations :

\begin{tabular}{|c|c|c|c|c|c|c|c|c|c|c|c|c|}
\hline$x=\mathrm{hr} . \mathbf{A} \cdot \mathrm{M}$. & . & 1 & 2 & 3 & 4 & 5 & 6 & 7 & 8 & 9 & 10 & 11 \\
\hline$y=\operatorname{deg} . \mathrm{F}$. & & +3.8 & +0.6 & -0.9 & -2.4 & -2.4 & -3.1 & +0.6 & +4.3 & +10.8 & +17.0 & +21.3 \\
\hline$x=1$ & . & 1 & 2 & 3 & 4 & 5 & 6 & 7 & 8 & 9 & 10 & 11 \\
\hline$y=\operatorname{deg} . F$. & • & +27.0 & 29.0 & 28.3 & 26.5 & 23.8 & 20.3 & 18.2 & 16.2 & 13.7 & 9.8 & 8.5 \\
\hline
\end{tabular}

To exhibit the variations in the temperature graphically we select two convenient units of length, one to represent one hour the other to represent one degree. We then plot each

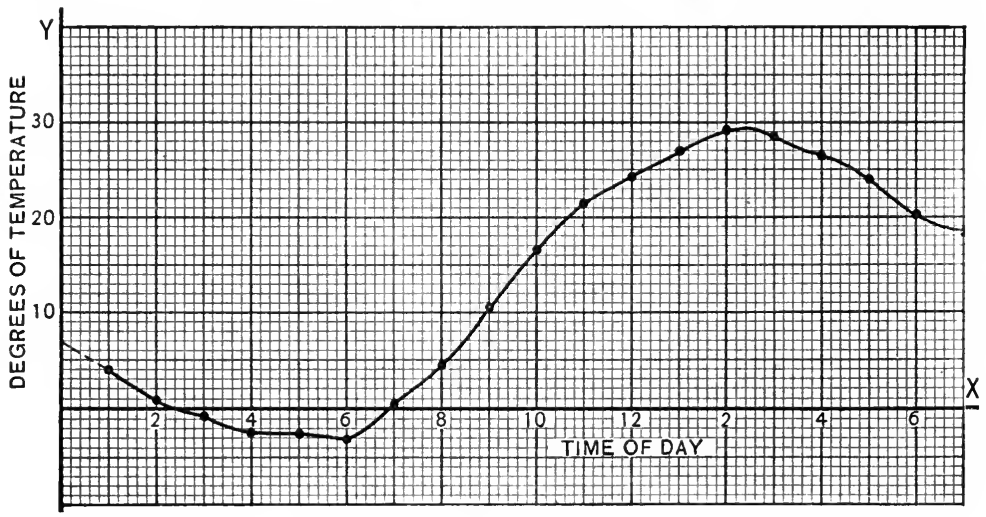

FIG. 5. - Temperature Graph. 
of the points $(1,+3.8),(2,+0.6),(3,-0.9)$, etc., the first number of each pair indicating the hour, the second the temperature at that hour. The question now arises whether we shall connect these points by a smooth curve or by a succession of straight lines. To answer this question we must consider the relation of the temperature to the time. Evidently the temperature changes gradually with the time; that is, the temperature can not change from one value to another without passing through all intermediate values. We shall therefore best represent the relation between temperature and time by connecting the points by a smooth curve as in figure 5 .

8.8. Advantages of the Graphic Method. An inspection of the graph in figure 5 shows :

(a) At what time of day the temperature reached a maximum and at what time it reached a minimum.

(b) The periods of the day during which the temperature was increasing and the periods when it was decreasing.

(c) The periods of the day when the temperature changed least.

(d) The periods of the day when the temperature changed most rapidly.

(e) The approximate time of day when any given temperature was reached.

$(f)$ The approximate temperature at any time that falls within the period of observation covered by the table, even though no observation was taken at that time. Thus it is easily seen that the temperature at noon must have been approximately $24^{\circ}$, though no observation was taken at noon.

$(g)$ The probable temperature at a time near but outside the hours given in the table of observations. We need only to prolong the graph and read off the ordinate of the point on the graph which has the given time for an abscissa. 
Thus by prolonging the curve in figure 5 for a short distance in both directions we find that the temperatures for the midnight preceding and following the hours were about $7^{\circ}$ and $9^{\circ}$ respectively.

8.9. Interpolation and Extrapolation. The process of finding the ordinate of an intermediate point on a graph from its abscissa, or vice versa, is called interpolation; when the point is on a prolonged portion of the graph it is called extrapolation. Both processes are exceedingly useful, but great caution must be exercised in their use. Both processes fail to give trustworthy results when the graph is very irregular. Extrapolated values are always more or less uncertain, since a graph frequently changes its course in the most unexpected manner. Yet extrapolation is useful in making forecasts even if these forecasts can not.be relied upon with certainty.

8.10. Broken Line Graphs. Characteristic Curves of Composition. It has been found that an author employing a given form of composition (narrative, description, drama, poetry, etc.) makes use of a definite percentage of words containing a given number of letters. In the case of Dryden's Essay on Satire we find :

No. of letters . . 1, 2, 3, 4, 5, 6, 7, 8, 9, 10, 10+, Frequency per cent 3.1, 19.5, 22.6, 17.7, 10.2, 8.1, 7.3, 4.9, 2.8, 2.0, 1.8.

To obtain a Characteristic Curve of Composition we plot points, using the number of letters as abscissas and their frequencies as ordinates. The points thus obtained should be connected by straight lines rather than by a smooth curve, because the ordinates (frequencies) do not admit of gradual change but change abruptly from one frequency to another. The resulting broken line graph (fig. 6) shows at a glance the distribution of words of varying lengths. It shows that three letter words are most numerous and that four letter, five 
letter, six letter words, etc., occur with diminishing frequencies, that two letter words occur oftener than four letter words and so on.

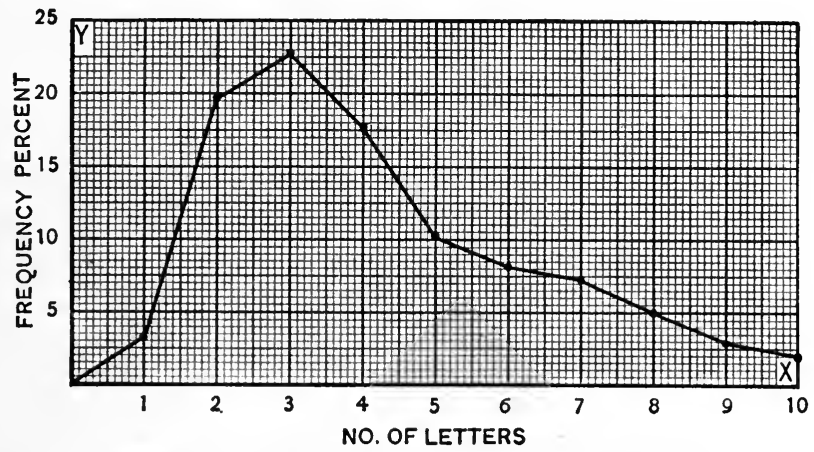

Fig. 6. - Characteristic Curve of Composition.

8.11. Extent of the Use of Graphs. Graphs are commonly used not only in mathematics but also in various other sciences, in engineering, in commerce and business, in transportation, in the study of sociological and economic questions. In mathematics their use is to visualize the relation between variable quantities, to illustrate abstract conclusions, to suggest hidden properties of quantities, and to obtain approximate solutions of many intricate problems. In the observational sciences graphs are constantly used to illustrate the laws of nature, engineers use them in their estimates and experimentations, inventors to show the workings of machinery, business men to follow the change in prices, cost of production, increase or decrease of sales, etc., economists to exhibit the changes in population, the changes in production, extent of unemployment, etc.

The methods employed for obtaining the data from which the graphs are constructed vary widely. In mathematics one 
of the sets of numbers is usually obtained from the other set by computation, in the physical sciences they are the result of exact measurements, in other sciences they are frequently the results of ordinary observation. In statistical investigations one or both sets of numbers are frequently the result of actual enumeration. In some cases instruments have been designed for constructing graphs mechanically without the intermediate process of first tabulating the observations or calculations. The thermograph, barograph, anemograph, oscillograph, and seismograph are among the more familiar of such instruments.

8.12. Coördinate Paper and Its Use. To facilitate the work of drawing graphs use is made of paper ruled in squares, called coördinate paper. The most common forms of coördinate paper are ruled to square millimeters, every fifth line and every tenth line (centimeter lines) being ruled somewhat heavier than the rest.

Before constructing a graph the coördinate axes should be drawn heavy and the scale used should be clearly marked on each axis. It is not necessary that the scale should be the same for both axes nor need the readings be necessarily zero at the origin. These are matters of judgment to be governed in part by the purpose for which the graph is drawn. Sometimes, in order to examine more minutely some portion of the graph, this portion is redrawn on a larger scale.

\section{EXERCISE 8}

1. Draw a pair of coördinate axes and indicate the positions of the following points :

$$
\begin{aligned}
& P_{1}=(3,5), \quad P_{2}=(7,2), \quad P_{3}=(-3,4), \quad P_{4}=(-2,-3), \\
& P_{5}=(5,-5), \quad P_{6}=(0,-3), \quad P_{7}=(-3,0), \quad P_{8}=(0,0), \\
& P_{9}=(-1,-1), \quad P_{10}=(2.5,-3.75) .
\end{aligned}
$$


2. A line is bisected by the origin. If the coördinates of one of its end points are $(3,5)$ what are the coördinates of the other end point? If one of the end points is $(3,-5)$ what is the other? How long is the line?

3. Draw a graph representing the statistics given in the following table:

Population of the United States

\begin{tabular}{l|r||r|r||c|c}
\hline \hline Year & Population & Year & Population & Y Ear & Population \\
\cline { 3 - 5 } 1800 & $5,308,000$ & 1840 & $17,069,000$ & 1880 & $50,156,000$ \\
1810 & $7,240,000$ & 1850 & $23,192,000$ & 1890 & $62,622,000$ \\
1820 & $9,638,000$ & 1860 & $31,443,000$ & 1900 & $75,569,000$ \\
1830 & $12,866,000$ & 1870 & $38,558,000$ & 1910 & $91,972,000$ \\
\hline
\end{tabular}

Suggestion. Let the year 1800 be represented by the origin; then the points on the $x$-axis whose abscissas are 10,20 , etc., will represent the years 1810,1820 , etc.

What does this graph teach regarding the change in the population of the United States? What was the probable population for each of the years 1855, 1894? Forecast the population in 1920. (Population in 1917, 103 millions, nearly.)

4. The following table contains the average duration (expectation) of life at various ages. Draw a graph using the age numbers for abscissas.

\begin{tabular}{|c|c|c|c|c|c|c|c|}
\hline AGE & Exp. & Age & Exp. & $\mathrm{AGE}_{\mathrm{E}}$ & Exp. & AGE & Exp. \\
\hline 0 & 53.6 & 10 & 53.4 & 40 & 29.3 & 70 & 9.4 \\
\hline 1 & 58.7 & 15 & 49.1 & 45 & 25.5 & 75 & 7.2 \\
\hline 2 & 59.2 & 20 & 44.9 & 50 & 21.7 & 80 & 5.4 \\
\hline 3 & 58.9 & 25 & 40.9 & 55 & 18.2 & 85 & 4.1 \\
\hline 4 . & 58.4 & 30 & 37.0 & 60 & 14.9 & 90 & 3.0 \\
\hline 5 & 57.7 & 35 & 33.1 & 65 & 12.0 & 95 & 2.3 \\
\hline
\end{tabular}


Discuss the variations in the expectation of life as revealed by the graph. Interpolate the expectation for each of the ages 27, 64, 72. Extrapolate the expectation for the age 100 . Find the age at which the expectation is the same as at birth. Find the age at which the expectation is equal to the age.

5. In surveying for a street the following field notes were taken. The distance between stations is 100 feet. The elevation is the distance in feet of the station above $(+)$ or below (-) a horizontal reference line known as the datum line. Draw a graph showing the profile of the ground surveyed.

\begin{tabular}{l|r||r|r||r|r||c|c}
\hline Sta. & Elev. & Sta. & Elev. & Sta. & Elev. & Sta. & Elev. \\
\cline { 2 - 5 } 1 & -5.2 & 6 & +6.1 & 11 & +3.9 & 16 & +24.6 \\
2 & -8.4 & 7 & +10.0 & 12 & +5.2 & 17 & +23.1 \\
3 & -9.5 & 8 & +7.9 & 13 & +6.0 & 18 & +24.0 \\
4 & -6.9 & 9 & +6.2 & 14 & +9.7 & 19 & +26.3 \\
5 & +0.3 & 10 & +4.0 & 15 & +16.8 & 20 & +24.0 \\
\hline
\end{tabular}

A street of uniform grade is to be graded between stations 1 and 20. By means of the graph and a straight line connecting stations 1 and 20 estimate the required cut $(+)$ or fill $(-)$ at each station.

6. In the following table $y$ represents tensile strength in tons per square inch of steel containing $x$ per cent of carbon.

\begin{tabular}{lllllllll}
$x$ & 0.14 & 0.46 & 0.57 & 0.66 & 0.78 & 0.80 & 0.87 & 0.96 \\
\hline$y$ & 28.1 & 33.8 & 35.6 & 40.0 & 41.1 & 45.9 & 46.7 & 52.7
\end{tabular}

Draw a graph and estimate the approximate percentage of carbon in steel that is to have tensile strength of 30 tons per square inch. Of 50 tons. What strength would you expect steel to have that contains 0.5 per cent of carbon? 
7. The following table gives the number of standard steel wire nails of a given length in a pound.

$\begin{array}{lllllllllll}\text { Length in inches } & 1 & 1.25 & 1.5 & 2 & 2.5 & 3 & 3.5 & 4 & 4.5 & 5\end{array}$ \begin{tabular}{lllllllllll}
\hline Number per pound & 876 & 568 & 316 & 181 & 106 & 69 & 49 & 31 & 24 & 18
\end{tabular}

Draw a graph and estimate the number of nails to the pound for all lengths at intervals of one quarter of an inch between the lengths two inches and four inches. How long would you expect wire nails to be which run 270 to the pound? 11 to the pound?

8. The Department of Agriculture gives the following statistics concerning the wheat crop of the United States during the past decade.

\begin{tabular}{|c|c|c|c|}
\hline Year & Acres & BUSHELS & Value \\
\hline 1906 & $47,305,000$ & $735,261,000$ & $\$ 490,333,000$ \\
\hline 1907 & $45,211,000$ & $634,087,000$ & $554,437,000$ \\
\hline 1908 & $47,557,000$ & $664,602,000$ & $616,826,000$ \\
\hline 1909 & $44,262,000$ & $683,350,000$ & - \\
\hline 1910 & $45.681,000$ & $635,121,000$ & $561,051,000$ \\
\hline 1911 & $49,543,000$ & $621,338,000$ & $543,063,000$ \\
\hline 1912 & $45,814,000$ & $730,267,000$ & $555,280,000$ \\
\hline 1913 & $50,184,000$ & $763,380,000$ & $610,122,000$ \\
\hline 1914 & $53,541,000$ & $891,017,000$ & $878,680,000$ \\
\hline 1915 & $60,469,000$ & $1,025,801,000$ & $942,303,000$ \\
\hline 1916 & $52,785,000$ & $639,886,000$ & $1,025,765,000$ \\
\hline
\end{tabular}

On the same sheet of coördinate paper construct three graphs, one showing the acreage, another the yield, and a third the value of the wheat crop for the ten years 1906-1917. (Suggestion. Round off the numbers to the nearest million.) Interpolate the value of the wheat crop for the year 1909. 


\section{LESSON IX - RELATED GRAPHS}

The graphic method may be employed to advantage in studying the relation between two or more sets of quantities which depend upon a common set. Using the common set as abscissas and each of the other sets in turn as ordinates, as many graphs may be constructed as there are sets of quantities to be compared. A comparison of the several graphs will show not only the relation between each set of quantities and the common set but also the relation between the various sets.

9.1. Wages and Purchasing Power. Suppose we wish to compare the change in wages during a certain period with the change in the purchasing power of wages during the same period, say for the period from 1890 to 1907. We may use for this purpose the following figures, which are taken from the Statistical Abstract of the United States, 1912, p. 296.

\begin{tabular}{c|c|c||c|c|c}
\hline Year & Earnings & Purch. Power & Year & Earnings & PUrch. Power \\
1890 & $101.0 \%$ & $98.6 \%$ & 1899 & $101.2 \%$ & $101.7 \%$ \\
1891 & 101.8 & 97.1 & 1900 & 104.1 & 103.0 \\
1892 & 101.3 & 99.4 & 1901 & 105.9 & 100.7 \\
1893 & 101.2 & 969 & 1902 & 109.2 & 98.5 \\
1894 & 97.7 & 98.0 & 1903 & 112.3 & 101.8 \\
1895 & 98.4 & 100.6 & 1904 & 112.2 & 100.4 \\
1896 & 99.5 & 104.2 & 1905 & 114.0 & 101.4 \\
1897 & 99.2 & 103.0 & 1906 & 118.5 & 102.4 \\
1898 & 99.9 & 101.2 & 1907 & 122.4 & 101.5 \\
\hline
\end{tabular}

The numbers both of wages and prices are computed on the basis of the average for the ten years 1890 to 1900 . By earn- 
ings is meant the full-time weekly earnings per employee and by purchasing power is meant the purchasing power as measured by the retail prices of food of the full weekly earnings per employee.

Figure $\tau$ contains two graphs plotted from the above data, one showing the fluctuation in wages, the other the fluctuation

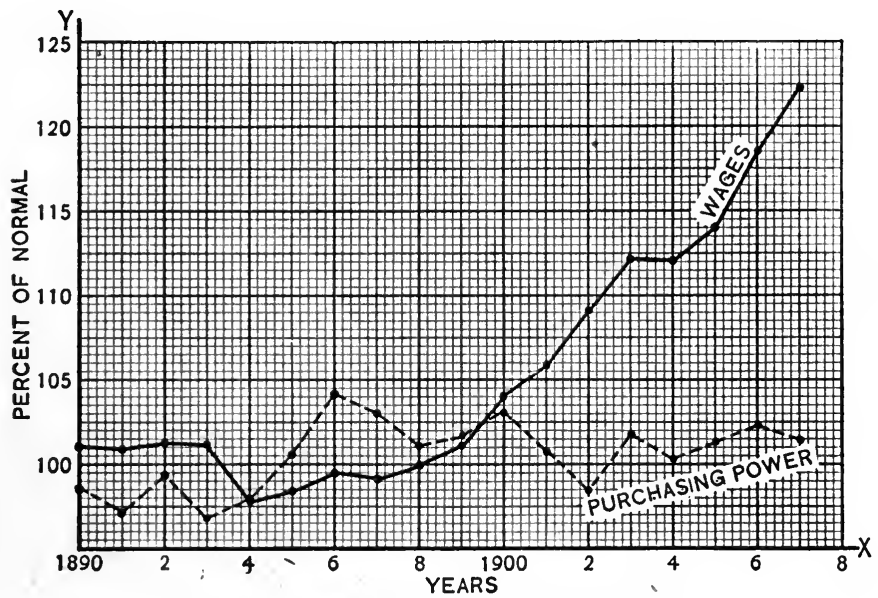

Frg. 7. - Wages and Purchasing Power.

of the purchasing power of wages. It is seen at a glance that since 1894 there has been a steady rise in wages without a corresponding increase of purchasing power. For the first four years of the period while wages were above normal (100\%) purchasing power was below normal, in 1894 wages and purchasing power were both below normal, during the next six years wages were on the whole below normal while purchasing power rose and remained above normal. Taking the period from 1896 to 1907 as a whole there has been a constant upward tendency in wages accompanied by a slightly downward tendency in purchasing power. 
9.2. Marriage Rate, Bank Clearings, Unemployment. As another illustration we show in figure 8 three graphs of which

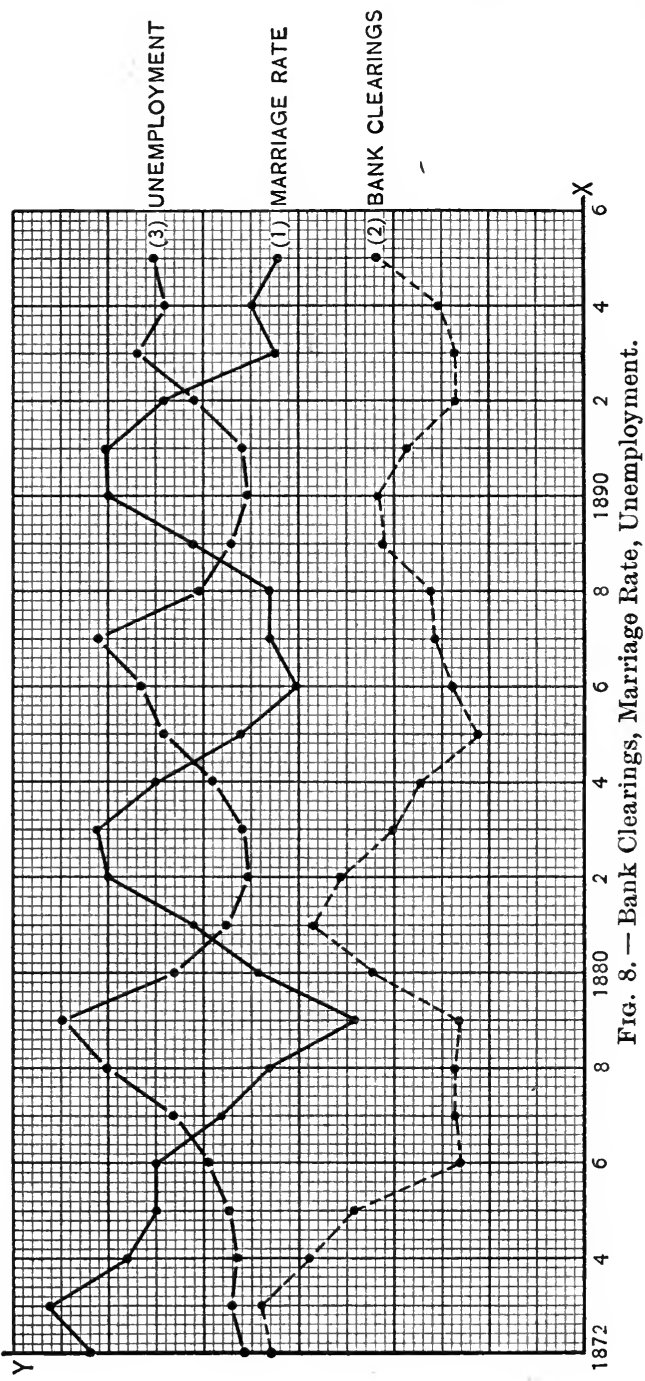

(1) gives the variation in the marriage rate, $(2)$ the bank clearings per capita, and (3) the variation in unemployment in England for the period 1872-1896. The curves are plotted from data which represent variations from the general trend. A comparison of these graphs shows beyond question a close relation between unemployment on the one hand and marriage rate and bank clearings on the other. As unemployment increases the marriage rate decreases and vice versa, to a maximum of either corresponds a 
minimum of the other. Notice also that the maximum and minimum points of the bank clearings graph slightly precede those of the marriage graph. Furthermore the fluctuations in each of the three graphs appears to be periodic, the period being approximately eight years.

\section{EXERCISE 9}

1. The following table contains certain United States post office statistics covering a period of 17 years. Represent the results graphically and discuss whatever relations you are able to observe between the three sets of figures.

\begin{tabular}{c|r|r|r}
\hline Y & No. OF CARriers & Daily Mileage & ANnUAL Cost \\
\hline 1901 & 4,300 & 100,000 & $\$ 1,750,000$ \\
1903 & 15,000 & 333,000 & $8,051,000$ \\
1904 & 25,000 & 553,000 & $12,645,000$ \\
1905 & 32,000 & 721,000 & $20,865,000$ \\
1906 & 35,000 & 820,000 & $25,012,000$ \\
1907 & 38,000 & 883,000 & $26,662,000$ \\
1908 & 39,000 & 891,000 & $34,372,000$ \\
1909 & 41,000 & 980,000 & $35,661,000$ \\
1910 & 41,000 & 993,000 & $36,915,000$ \\
1911 & 42,000 & $1,008,000$ & $37,126,000$ \\
1912 & 42,000 & $1,022,000$ & $41,859,000$ \\
1913 & 43,000 & $1,029,000$ & $45,643,000$ \\
1914 & 44,000 & $1,050,000$ & $47,377,000$ \\
1915 & 44,000 & $1,073,000$ & $52,566,000$ \\
1916 & 43,000 & $1,063,000$ & $51,952,000$ \\
1917 & 43,000 & $1,113,000$ & $52,420,000$ \\
\hline
\end{tabular}

The numbers have all been rounded off to the nearest thousand.

2. The following table was compiled by a state board of health. $T$ stands for the percentage of reports of the month in which typhoid fever was mentioned as prevalent, $W$ stands for the number of feet of the level of the water in the wells 


\begin{tabular}{|c|c|c|c|c|c|c|c|c|}
\hline MoNTH & $T$ & $w$ & Month & $T$ & $W$ & MoNth & $T$ & $W$ \\
\hline Jan. & 10.1 & 17.8 & May & 5.0 & 16.0 & Sept. & 18.0 & 18.9 \\
\hline Feb. & 7.2 & 17.3 & June & 6.6 & 16.6 & Oct. & 23.5 & 19.1 \\
\hline March & 5.0 & 16.6 & July & 7.5 & 17.5 & Nov. & 19.1 & 19.1 \\
\hline April. . & 4.4 & 16.3 & Aug. .... & 11.1 & 18.3 & Dec. & 18.4 & 18.5 \\
\hline
\end{tabular}

below the high water mark. Draw both graphs on the same sheet of paper and state your conclusion as to the relation between the two sets of facts.

3. The following table is taken from a work on astronomy. The first column contains the year number, the second marked $(N)$ contains the number of days in the year when no sunspots

\begin{tabular}{|c|c|c|c|c|c|c|c|}
\hline Year & $(N)$ & $(G)$ & $(V)$ & YeAR & $(N)$ & $(G)$ & $(V)$ \\
\hline 1826 & 22 & 118 & $9.75^{\prime}$ & 1848 & 0 & 330 & $11.15^{\prime}$ \\
\hline 1827 & 2 & 161 & 11.33 & 1849 & 0 & 238 & 10.64 \\
\hline 1828 & 0 & 225 & 11.38 & 1850 & 2 & 186 & 0.44 \\
\hline 1829 & 0 & 199 & 14.74 & 1851 & 0 & 151 & 8.32 \\
\hline 1830 & 1 & 190 & 12.13 & 1852 & 2 & 125 & 8.09 \\
\hline 1831 & 3 & 149 & 12.22 & 1853 & 3 & 91 & 7.09 \\
\hline 1832 & 49 & 84 & - & 1854 & 65 & 67 & 6.81 \\
\hline 1833 & 139 & 33 & 一 & 1855 & 146 & 79 & 6.41 \\
\hline 1834 & 120 & 51 & 一 & 1856 & 193 & 34 & 5.98 \\
\hline 1835 & 18 & 173 & 9.57 & 1857 & 52 & 98 & 6.95 \\
\hline 1836 & 0 & 272 & 12.34 & 1858 & 0 & 188 & 7.41 \\
\hline 1837 & 0 & 333 & 12.27 & 1859 & 0 & 205 & 10.37 \\
\hline 1838 & 0 & 282 & 12.74 & 1860 & 0 & 211 & 10.05 \\
\hline 1839 & 0 & 162 & 11.03 & 1861 & 0 & 204 & 9.17 \\
\hline 1840 & 3 & 152 & 9.91 & 1862 & 3 & 160 & 8.59 \\
\hline 1841 & 15 & 102 & 7.82 & 1863 & 2 & 124 & 8.84 \\
\hline 1842 & 64 & 68 & 7.08 & 1864 & 4 & 130 & 8.02 \\
\hline 1843 & 149 & 34 & 7.15 & 1865 & 25 & 93 & 8.14 \\
\hline 1844 & 111 & 52 & 6.61 & 1866 & 76 & 45 & 7.65 \\
\hline 1845 & 29 & 114 & 8.13 & 1867 & 195 & 25 & 7.09 \\
\hline 1846 & 1 & 157 & 8.81 & 1868 & 23 & 101 & 8.15 \\
\hline 1847 & 0 & 257 & 9.55 & & & & \\
\hline
\end{tabular}




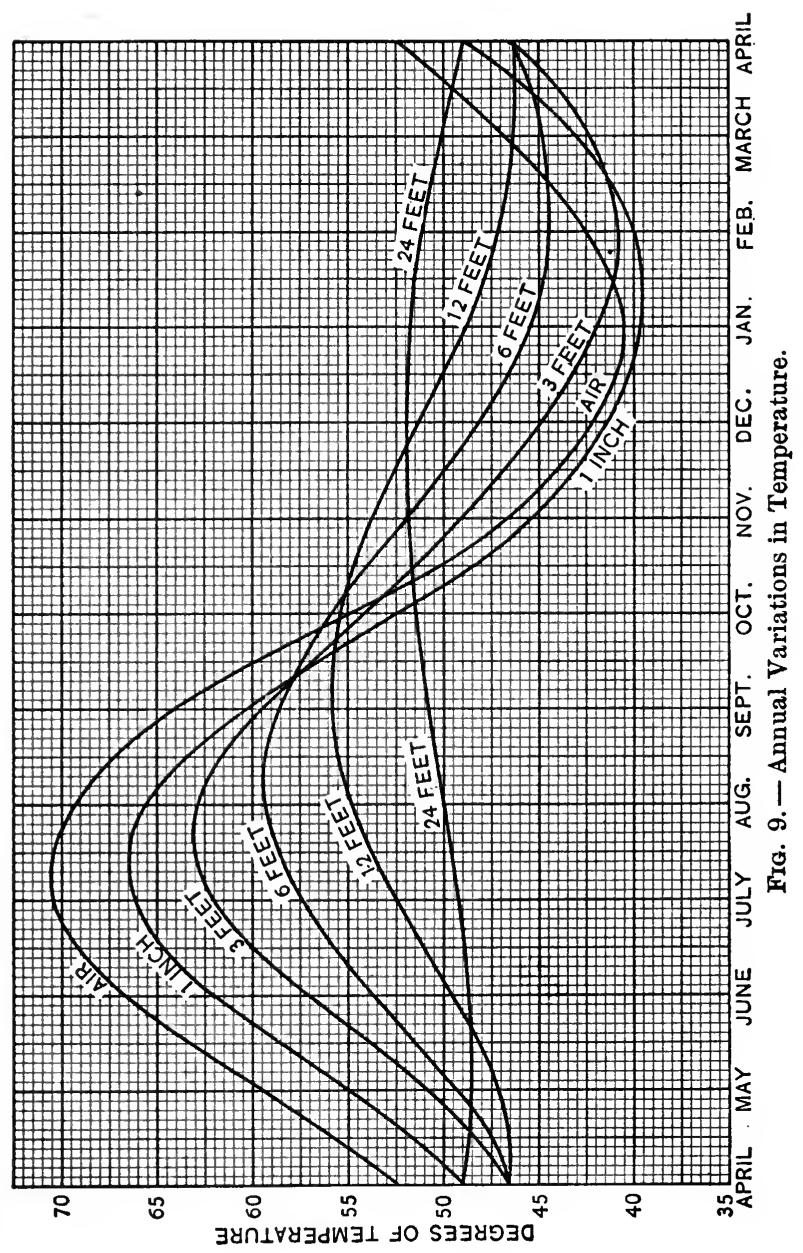


were visible on the sun, the third column marked $(G)$ contains the number of new groups of spots that appeared on the sun during the year, the fourth column marked $(V)$ contains the mean daily variation in the declination of the magnetic needle during the year. Draw the three graphs and interpret the results. Also interpolate the missing numbers in the column $(V)$.

4. The manufacturer of a patented utensil finds the following relation between the selling price of the utensil and the number he can sell at that price :

Selling price in cents . 5

$\begin{array}{llllllll}\text { Number sold } & \text {. } & 200,000 & 190,000 & 150,000 & 80,000 & 30,000 & 10,000\end{array}$

Draw a graph and estimate the number he may expect to sell at 12 cents each. At 17 cents each. Assuming that it costs him 4 cents to manufacture the article, draw a graph showing his total profit at any given selling price between five cents and thirty cents. At what figure should he fix the selling price so as to yield him the largest profit?

Ans. 175,$000 ; 110,000 ; 15$ cents.

5. Figure 9 contains six graphs representing the average annual variation in temperature at various depths below the surface of the earth at London, England. The average is computed for a period of 14 years. Discuss as completely as you can the effect of depth on the variation in temperature and the relation between the maximum and minimum temperatures to the seasons of the year. 


\section{LESSON $\mathrm{X}$-STRAIGHT LINE GRAPHS}

10.1. The Straight Line Graph. Because of the ease with which it may be drawn and the frequency with which it occurs the straight line graph is of special interest and importance. A straight line is determined by any two of its points, if therefore it is known that the graph which is to be drawn is a straight line, it is sufficient to plot two of its points and connect these points by a straight line.

10.2. Graphs of Conversion Tables. Tables which express units of one system of measure in terms of units of another system are called conversion tables and any such table may

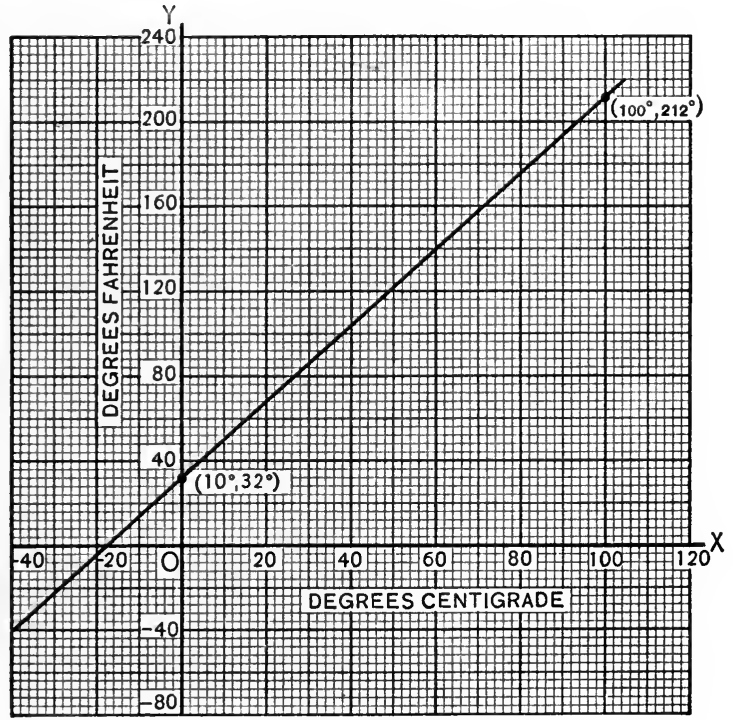

Fig. 10. - Centigrade-Fahrenheit Conversion Graph. 
be represented by a straight line graph. Consider for example the following table, in which $x$ represents temperature readings in degrees Centigrade and $y$ the corresponding readings in degrees Fahrenheit.

$$
\begin{array}{lrrrrrrrrrrr}
x=\text { deg.C. } & 0 & 10 & 20 & 30 & 40 & 50 & 60 & 70 & 80 & 90 & 100 \\
\hline y=\text { deg.F. } & 32 & 50 & 68 & 86 & 104 & 112 & 140 & 158 & 176 & 194 & 212
\end{array}
$$

We plot any two points, as for instance the two points $(0,32)$ and $(100,212)$. The straight line connecting these points is the required graph (fig. 10).

Every point on this graph represents some temperature, the abscissa of the point gives the measure of this temperature in degrees Centigrade, its ordinate in degrees Fahrenheit. The graph therefore enables us to convert any temperature reading given in degrees Centigrade into degrees Fahrenheit and vice versa.

10.3. Price Schedules. The following is a schedule of rates issued by a city gas company. 500 cubic feet or less . . . . . . . . . . . $\$ 0.50$ Excess above 500 cubic feet, per 100 cubic feet . • .06 Excess above 5000 cubic feet, per 100 cubic feet $\quad$ • $\quad .04$

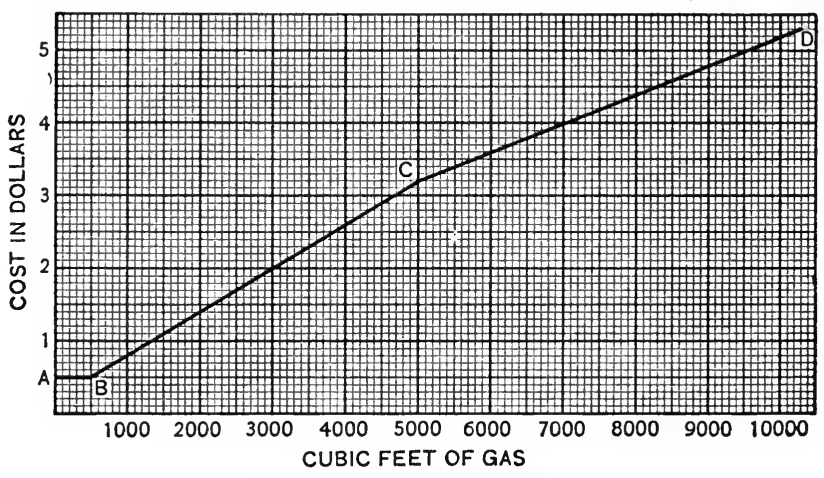

Frg. 11. - Gas Price Schedule. 
Draw a graph which will show the cost of any given amount of gas consumed.

Using the number of cubic feet as abscissas and the corresponding cost as ordinates we obtain the graph in figure 11 which consists of three straight lines. $A B$ gives the cost of any number of cubic feet up to $500, B C$ the cost of any number of cubic feet between 500 and 5000 , and $C D$ the cost of any number of cubic feet above 5000 cubic feet. The graph furthermore shows the quantity of gas that may be purchased at any given cost. Thus it is seen that $\$ 4.40$ will purchase 8000 cubic feet, since 8000 is the abscissa of that point on the graph whose ordinate is 4.40 .

10.4. Time Schedules. A case of considerable practical importance is the graphical representation of time schedules which is employed in more or less modified form in many railroad offices. Let us consider an imaginary schedule like the following :

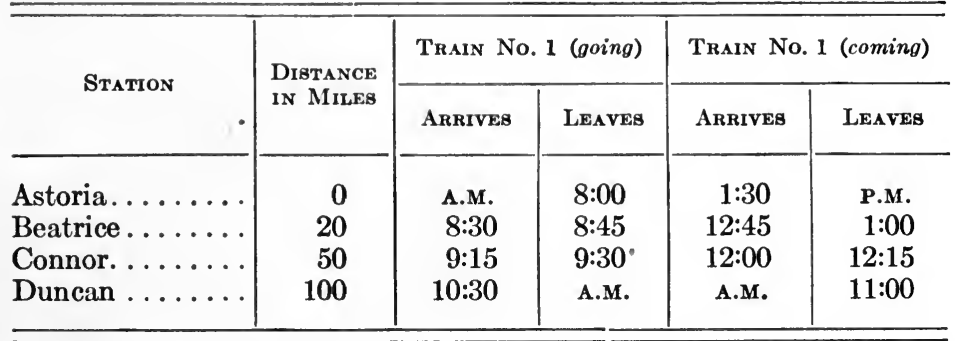

The speed of the train between stations is assumed to be uniform. Let us take the hours after 8:00 o'clock for abscissas and the distances of the stations from Astoria as ordinates (figure 12). Then the straight line $A B$ connecting the end points $(0,8: 00)(20,8: 30)$ represents the progress of the train between Astoria and Beatrice; the ordinate of any point 
on this line will give the distance which the train was from Astoria at the time indicated by its abscissa. The stop of the train at Beatrice is represented by the horizontal line $B B^{\prime}$, its

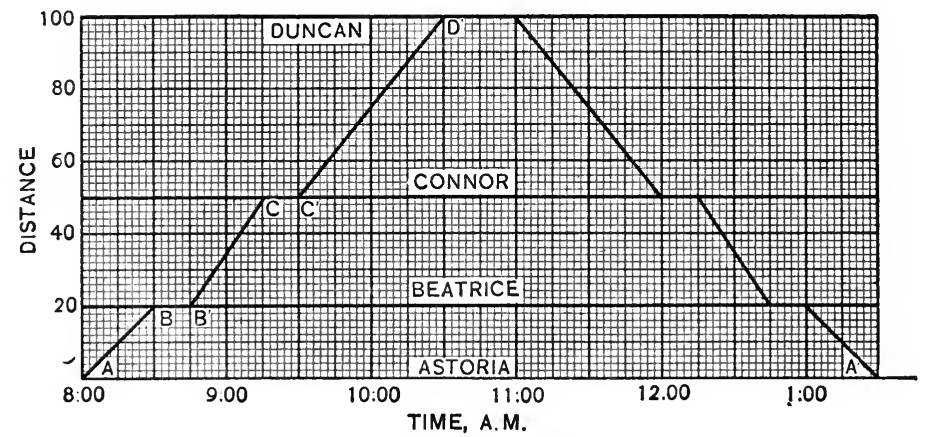

Fig. 12. - Train Time Schedule.

progress between Beatrice and Connor by the line $B^{\prime} C$, its stop at Connor by the horizontal line $C C^{\prime \prime}$, etc. Similarly the return trip of the train is represented by the broken line $D^{\prime} A^{\prime}$.

The advantage of a graph like that in figure 12 is that it shows at a glance not only the exact position of a train at any given time, but also the exact time at which the train passed any point on its way.

\section{EXERCISE 10}

1. By means of the graph in figure 10 express $60^{\circ}$ Centigrade in degrees Fahrenheit. Express $10^{\circ}$ Fahrenheit in degrees Centigrade. Express $-12^{\circ} \mathrm{F}$. in degrees $\mathrm{C}$. What temperature is expressed by the same number in both scales? For what temperature are the two readings numerically equal but opposite in sign?

2. Construct a graph for converting mils into minutes.

3. Construct a graph for converting kilograms into pounds and conversely, assuming that $1 \mathrm{lb}$. $=0.45 \mathrm{~kg}$. 
4. Draw a graph showing the wages due at 50 cents per hour for any number of hours up to 10 hours. On the same sheet draw other graphs showing the wages due at 60 cents, 70 cents, etc., up to $\$ 1$ per hour for any number of hours up to 10 hours.

5. Draw a graph showing the interest due on $\$ 100$ at the rates of 5 per cent, 6 per cent, 7 per cent, 8 per cent, 9 per cent, 10 per cent for any time less than one year.

6. Draw a graph showing the interest due for one year at each of the rates given in problem 5 on any sum less than $\$ 100$.

7. The cost of printing a circular letter is $\$ 10$ for 500 or less copies, and 50 cents additional 10 100 copies or fraction thereof in excess of 500 copies. Construct a graph showing the cost of any number of letters up to 1000 .

8. A graduated income tax provides for exemption from tax of incomes up to $\$ 3000,5$ per cent on the excess above $\$ 3000$, and 10 per cent on the excess above $\$ 10,000$. Construct a graph showing the tax on any income up to $\$ 50,000$. 


\section{LESSON XI-GRAPHS AND PROBLEM SOLVING}

11.1. Simultaneous Straight Line Graphs. There is another important use to which graphs, and especially straight line graphs, may be put. By using a common scale and the same coördinate axes in the construction of the several graphs that are suggested in a given statement of facts or conditions, questions can frequently be answered by inspection or by the measurement of certain lines or angles which, without the aid of the graphs, involve considerable computation. Indeed questions may be asked and answered by the aid of graphs which, without such aid, are beyond the mathematical ability of many students.

\subsection{Graphical Solution of Arithmetic Problems. The} following problem is typical of a vast class of problems that can be solved by the graphic method.

$\mathrm{A}$ and $\mathrm{B}$ are two towns 18 miles apart. $\mathrm{P}$ leaves $\mathrm{A}$ at 8:00 o'clock and walks till noon toward $B$ at at the rate of $2 \frac{1}{2}$ miles per hour. From 12:00 till 1:00 o'clock he rests, then he walks back toward A for two hours at the rate of 3 miles per hour. Now he turns again and without delay continues his journey toward B, arriving at B at 7:00 o'clock. A second traveler $Q$ leaves B at 9:00 o'clock and walks toward A 10 miles by $11: 30$, then after a delay of $3 \frac{1}{2}$ hours completes his journey to $\mathrm{A}$ at the rate of 3 miles per hour.

What was P's final rate of travel? At what time did $Q$ reach $A$ ? When and where $\operatorname{did} P$ meet $Q$ ? When were they 10 miles apart?

Suppose P's and Q's graphs have been drawn on the same sheet, as in figure 13. The figure shows that P's final rate 
must have been $\frac{14}{4}$ or $3 \frac{1}{2}$ miles per hour and that $Q$ reached $\mathrm{A}$ at 5:40 P.M. The times and places where $\mathrm{P}$ met $\mathrm{Q}$ are

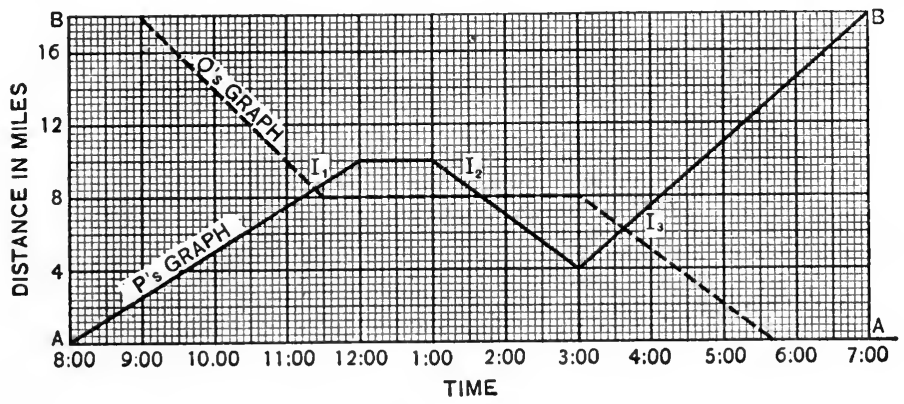

Fia. 13.

given by the coördinates of the points $I_{1}, I_{2}, I_{3}$, in which the two graphs intersect, and the times when they were 10 miles apart are given by the abscissas of those points on the two graphs, whose ordinates differ by 10 .

It should be observed that the answers to problems solved by the graphic method are necessarily only approximations of the true results. The graphic method therefore fails when absolute accuracy is required.

11.3. Comparison of Price Lists. A merchant wishes to send out a certain number of circular letters. He finds that to have them typewritten will cost him 7 cents each copy; to make them from a stencil will cost 1 cent per copy with an initial cost of $\$ 1$ for making the stencil. If more than 500 copies are to be made by this method a new stencil must be made. To print them from type will cost $\$ 3.50$ for the first 100 or less and 50 cents extra for each additional 100 or frac. tion thereof.

Which of these methods is most economic for 20 copies? For 200 copies? For 400 copies? For 450 copies? For 500 
copies? For what number is the cost the same by the first two methods? By the last two methods? By the first and third methods? What is the difference in the cost of 250 copies by the last two methods? If the cost of the letters is not to exceed $\$ 5.50$ what method should be employed in making the letters?

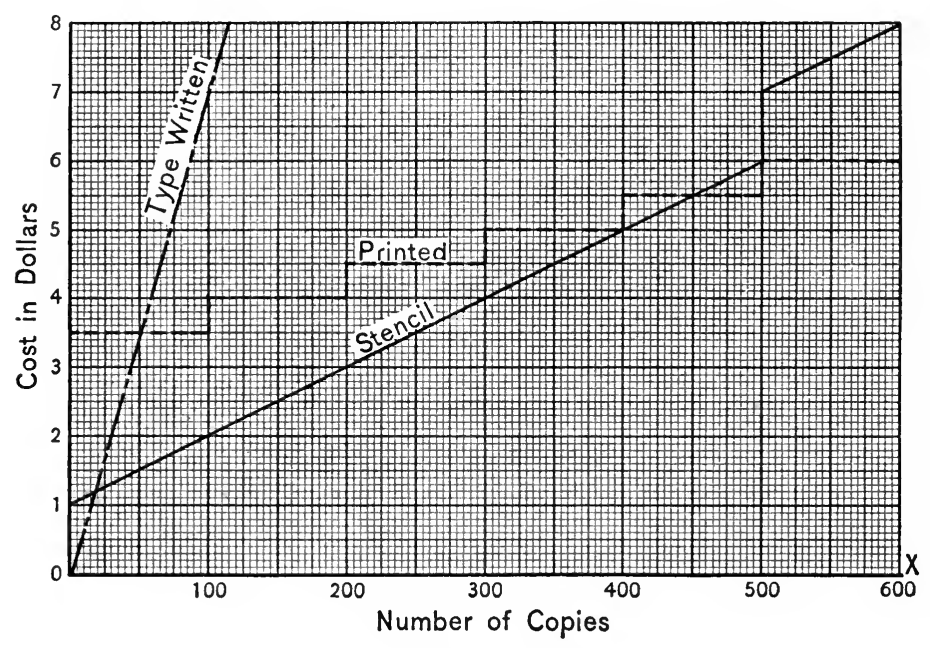

Fia. 14.

All of these questions can be readily answered by referring to the three graphs in figure 14.

11.4. Adjustment of Train Schedules. It was shown in 10.4 how the schedule of a single train, as train No. 1, may be represented by a graph. Suppose now that a second train is to be scheduled to run on the same track and between the same stations as train No. 1. It is plain that the movement of the second train must be so controlled that the two trains may pass each other at stations or else sidings must be put in at 
proper distances for this purpose. After the second train's schedule has been properly adjusted and its graph drawn, a third train may be similarly scheduled and its graph constructed. Such a composite graph will show at a glance the relative positions of the various trains at any given time, the points where the trains pass each other, the times of arrival and departure at any given station, etc.

In practice, instead of lines, different colored elastic threads are frequently used to indicate the course and progress of the various trains. These threads are fastened to pins so that their relative position can be readily adjusted to correspond to the changes in the moving trains, for at times some trains must be speeded up, others retarded, as certain exigencies may require.

Moreover, the graph used in actual practice contains certain information with which we are not concerned, such as grades, curves, switches, sidetracks, signals, etc.

The student should construct for himself the graphs representing the following time schedules and check his work by comparing it with figure 15. He should then be able to answer at sight the questions asked in connection with each schedule.

Example. Train No. 1 runs according to the following table :

\begin{tabular}{l|r|c|c||c|c|c|c}
\hline Sta. & Dist. & Arrives & Departs & Sta. & Dist. & Arrives & Departs \\
\cline { 5 - 7 } & & & & & & & \\
A & 0 & A.M. & $6: 00$ & F & 70 & $10: 27$ & $10: 45$ \\
B & 15 & $6: 45$ & $7: 00$ & G & 80 & $11: 14$ & $11: 15$ \\
C & 35 & $8: 00$ & $8: 06$ & H & 95 & $12: 00$ & $12: 09$ \\
D & 43 & $8: 42$ & $8: 45$ & I & 120 & $1: 27$ & \\
E & 50 & $9: 30$ & $9: 33$ & & & & \\
\hline
\end{tabular}

Between which stations did this train run slowest? 





Train No. 2 is to be scheduled so as to leave station I at 9:30 A.M. and to reach station $\mathrm{F}$ three minutes before and leave three minutes after the arrival of train No. 1, then to continue at, the same rate as before without further stops to A. Required the speed of train No. 2 and its time of arrival at A.

Train No. 3 is to be scheduled from $A$ to $I$ so as to pass train No. 1 at station $\mathrm{C}$ and to allow train No. 2 to pass it at station $\mathrm{H}$. When must this train leave $\mathrm{A}$, what is its speed, and when will it arrive at I, assuming that its speed is uniform, that it makes a 6 -minute stop at $\mathrm{H}$ but no other stops?

Train No. 4 is to leave $H$ at 7:15 A.M. The train is to make 20 miles an hour with 15-minute stop at each station. Sidings are to be put in wherever necessary to let the other trains pass. Determine the locations of these sidings, and the time when train No. 4 will arrive at A.

\section{EXERCISE 11}

1. A city has two competing gas companies. The first company charges $\$ 1.25$ per 1000 cubic feet, the other charges at the rate of $\$ 1.50$ for the first 1000 cubic feet and $\$ 1.20$ for the excess above 1000 cubic feet.

Represent the two sets of rates graphically. For what amount of gas consumed will the charges by the two companies be the same? What will be the difference in the charges by the two companies to a consumer who uses 3000 cubic feet? 10,000 cubic feet? How much more gas will $\$ 3.50$ pay for in the one company than in the other?

2. Two firms sell the same article. One charges 5 cents apiece with a discount of 20 per cent on purchases exceeding $\$ 5$, the other firm charges 6 cents apiece with a discount of 25 per cent on purchases in excess of $\$ 3$. Draw the two graphs. Which of the two companies should I patronize in 
ordering 40 articles? 60 articles? 120 articles? For what number of articles would the charges by the two firms be the same? Which firm gives the better rates on purchases amounting to $\$ 5$ ?

3. A train leaves Seattle for Portland at 8:00 A.M. making 30 miles per hour. A second train leaves Seattle for Portland on a parallel track at 9:00 A.M. making 50 miles per hour.

When and where will the second train overtake the first? How far will the two trains be apart at 11:30 A.M.? When will they be 30 miles apart? What time will elapse between the passing of the trains at a station 110 miles from Seattle? Ans. 10:30 A.M., 75 mi.; 20 mi. ; 12:00 M.; $28 \mathrm{~min}$.

4. $\mathrm{P}$ and $\mathrm{Q}$ start at the same time, $\mathrm{P}$ to walk from $\mathrm{A}$ to $\mathrm{B}$ and $\mathrm{Q}$ to walk from $\mathrm{B}$ to $\mathrm{A}$. They reach their destinations 4 and 5 hours respectively after they met on the road. How long did it take each to make his trip? Ans. P, $8 \mathrm{hr} .28 \mathrm{~min}$.

5. A boy walks to the top of a mountain at the rate of 1 mile per hour and returns the same way at the rate of 4 miles per hour. He was gone 11 hours. Assuming that he spent 1 hour in resting, how far did he walk?

6. If oats are worth 60 cents a bushel and wheat $\$ 1$ a bushel, what proportion of each should a mixture contain which sells at 90 cents a bushel?

Ans. $1: 3$.

7. A man bought 100 acres of land for $\$ 4800$. Part of the land cost him $\$ 40$ per acre, the remainder $\$ 60$ per acre. How many acres of each kind of land did he buy?

8. A and B start from the same point on a circular track to run in opposite directions. A makes a lap in four minutes, $\mathrm{B}$ in five minutes. When will they meet the first time? The second time? The third time? 
9. At what times between 12 and 6 o'clock are the minute and hour hands of a clock together? When are they at right angles? When do they point in exactly opposite directions?

10. Suppose that it takes six days to sail from New York to Liverpool, and that a boat leaves New York for Liverpool every morning at 8 o'clock. How many boats would a traveler meet in going from Liverpool to New York? When would he meet each boat, assuming that the boats sail at a uniform rate, and that he leaves Liverpool at noon?

11. A railroad train after traveling for 1 hour meets with an accident which delays it 1 hour, after which it proceeds $\frac{3}{5}$ of its former rate and arrives at its destination 2 hours and 30 minutes late. Had the accident occurred 60 miles farther on the train would have been only 1 hour 45 minutes late. What distance did the train go, and what was its original speed? Ans. $173.3 \mathrm{mi} ., 53.3 \mathrm{mi}$. 


\section{LESSON XII - CURVE PLOTTING}

12.1. The Equation of a Graph. It was stated in $\mathbf{8 . 1 1}$ that one of the two sets of numbers employed in the construction of a graph may have been obtained from the other set by computation. When such is the case the two sets of numbers must bear an exact relation to each other and this relation can always be expressed by means of an equation. This equation is known as the equation of the graph.

Suppose the two sets of numbers are

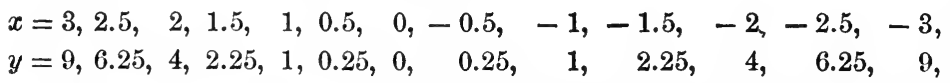

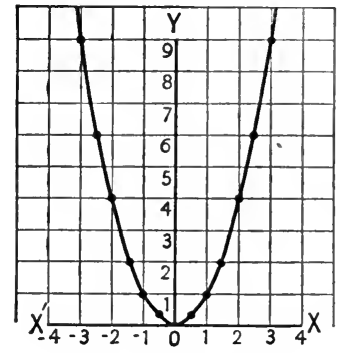

FIG. 16. $-y=x^{2}$. where the numbers of a second set are the squares of the numbers of the first set. The graph obtained by using the values of $x$ as abscissas and the values of $y$ as ordinates of points is shown in figure 16. This graph is completely characterized by the property that the ordinate, $y$, of any point on the graph is equal to the square of the abscissa, $x^{2}$, of that point. For this reason we call $y=x^{2}$ the equation of the graph, the graph is called the graph of $y=x^{2}$.

Definition. The equation of a graph is any statement in algebraic form of the exact relation between the coördinates of each and every point of the graph.

12.2. Mathematical Curves. Evidently not every graph has an equation for in many cases no exact relation exists between the coördinates of the points. In other cases, even though such a relation exists, it may not be known. Any 
graph whose course can be defined by an equation is called a mathematical curve. This definition gives a much broader significance to the word curve than the ordinary use of the word implies. A mathematical curve may consist of two or more disconnected branches, it may contain isolated points, it may consist of one or more straight lines.

To plot the curve of a given equation means to construct its graph by means of points.

\subsection{Equivalent Equations Have the Same Graph.} Equivalent equations are equations which are satisfied by the same sets of values of the unknowns and must therefore lead to the same graph. For example the equations $y=x^{2}, x^{2}=y$, $y-3=x^{2}-3,3-y=3-x^{2}, y-4=(x+2)(x-2)$, have the same graph.

12.4. To Plot the Curve of a Given Equation. In plotting curves it is important that the beginner observe the following directions :

(a) Solve the given equation for $y$, that is, express $y$ in terms of $x$.

(b) Assign numerical values to $x$ and compute the corresponding values of $y$.

(c) Plot points, using the values of $x$ as abscissas and the corresponding values of $y$ as ordinates.

(d) When enough points have been plotted to suggest the entire course of the curve, connect them by as smooth a curve as possible. This curve is the curve or graph of the given equation.

Remarks. Frequently it is impossible to construct the entire curve owing to the fact that the curve is endless. In that case the student should nevertheless form a mental image of those portions of the curve that fall beyond the confines of his paper. 
Negative as well as positive values of $x$ should be considered, and large as well as small values of $x$.

$y$ may have two (and even more) values for some or all values of $x$. In that case there will be two (or more) points. having the same abscissa but different ordinates.

For some values of $x$ the corresponding value of $y$ may involve the square-root of a negative number. Such values of $y$ are called imaginary and are disregarded in plotting curves.

If, after the points have been plotted, there remains a doubt as to which points should be connected the remedy is to plot more points in the doubtful region.

Sometimes it is more convenient to solve the given equation for $x$ than for $y$. In that case we assign arbitrary values to $y$ and compute the corresponding values of $x$. After that, we proceed as directed in $(c)$ and $(d)$.

12.5. Example. To plot the curve of the equation $(y-x)^{2}=x+4$.

(a) On solving the equation for $y$ we obtain $y=x \pm \sqrt{x+4}$.

(b) We next assign to $x$ the successive values $0,1,2,3$, 4, etc., and also the negative values $-1,-2,-3,-4$, etc., and compute the corresponding values of $y$. It is best to arrange the results in tabular form as follows :

$$
\begin{aligned}
& x=0, \quad+1, \quad+2, \quad+3, \quad+4, \quad+5, \quad+6, \quad \text { etc. } \\
& y=+2, \quad+3.24,+4.45,+5.65,+6.83,+8,+9.16 \text {, etc., } \\
& \text { or }-2,-1.24,-0.45,+0.35,+1.17,+2,+2.84 \text {, etc., } \\
& x=-1, \quad-2, \quad-3, \quad-4, \quad-5 \text { or less, } \\
& y=+0.73,-0.59,-2, \quad-4, \quad \text { imaginary, } \\
& \text { or }-2.73,-3.41,-4,-4 \text {. }
\end{aligned}
$$

(c) We now plot these points, observing that, since to every value of $x$ belong two values of $y$, the curve is made up of pairs of points, the points of each pair having a common abscissa. These points clearly suggest the trend of the curve except in the neighborhood to the right of $x=-4$. Any uncertainty like this we remove by plotting a few more points where needed. Thus we find that when $x=-3.5, y$ has the two 
values -2.79 and -4.21 ; when $x=-3.75, y$ has the two values -3.25 and -4.25 . On plotting these points, any doubt that previously remained will be removed.

(d) It only remains to connect the points already plotted by a smooth curve as shown in figure 17. Of course the curve actually drawn is only a portion of the entire curve, for any value of $x$ greater than 6 causes the corresponding values of $y$ to be real. In other words, both branches of the curve extend indefinitely to the right beyond the confines of our paper. But we can form a clear mental image of these branches even though we can-

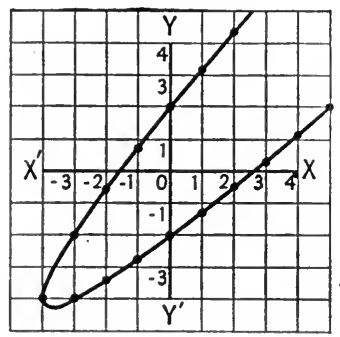

FIG. 17. $-y=x \pm \sqrt{x+4}$. not draw them. Each branch tends to become more and more nearly straight and the distance between the two branches becomes larger and larger as we extend them toward the right.

\section{EXERCISE 12}

1. Given the two sets of numbers :

$$
\begin{aligned}
& x=5, \quad 4, \quad 3, \quad 1, \quad 0, \quad-1, \quad-2, \quad-3, \quad-4, \quad-5 \text {, } \\
& y=7, \quad 6,5, \quad 3,2, \quad 1, \quad 0, \quad-1, \quad-2, \quad-3 \text {; }
\end{aligned}
$$

construct the graph and write its equation.

2. Given the two sets of numbers :

$$
\begin{aligned}
& x=4, \quad 2, \quad 1, \quad \frac{1}{2}, \quad \frac{1}{4}, \quad-\frac{1}{4}, \quad-\frac{1}{2}, \quad-1, \quad-2, \quad-4, \\
& y=\frac{1}{4}, \quad \frac{1}{2}, \quad 1, \quad 2, \quad 4, \quad-4, \quad-2, \quad-1, \quad-\frac{1}{2}, \quad-\frac{1}{4} ;
\end{aligned}
$$

construct the graph and write its equation.

Draw the graphs for each of the following equations :
3. $y-x=0$.
4. $y=x+1$.
5. $y=1-x$.
6. $y=3 x+2$.
7. $y=3-2 x$.
8. $y=x^{2}+1$.
9. $y=x^{2}-1$.
10. $y=1-x^{2}$.
11. $y^{2}=x$.
12.' $y^{2}=x-1$.
13. $y=1 / x$.
14. $y=1 / x^{2}$.
15. $y=1 /(1-x)$.
16. $y=1 /(x+2)$.
17. $y=x /(x-1)$ 
18. One way of stating the law of falling bodies is $v=g t$, where $v$ is the velocity in feet per second, $t$ the number of seconds which the body has been falting, and $g=32.2$. Construct a graph giving the velocity for any time up to 10 seconds.

19. The amount $(A)$ of a principal $(P)$ put out on interest at the rate $r$ for $t$ years is given by the formula $A=P(1+r t)$. Draw two graphs, one showing the amount of $\$ 1$ at 6 per cent for any time up to 20 years, the other showing the amount of $\$ 1$ for 10 years at any rate up to 10 per cent.

20. Mendeeleff's formula for the surface tension of a liquid at temperature $\theta$ is $t=a-b \theta$, where $a$ and $b$ are two constants. Draw a graph showing the surface tension at any temperature up to $100^{\circ}$ when $a=.5$ and $b=.25$.

21. The solubility of potassium chloride (s) in 100 parts of water at temperature $(\theta)$ is approximately given by the formula $2 s=\theta+57$. Draw a graph showing the solubility at any temperature up to $100^{\circ}$. 


\section{LESSON XIII-MAXIMA AND MINIMA. AREAS}

13.1. Definition of a Function. Whenever two variables are so related that the value of one depends upon the value of the other, each is said to be a function of the other. The area of a circle is a function of its radius; the interest received on capital invested is a function of the sum invested, it is also a function of the rate of interest earned; the distance a train moves is a function of the time, it is also a function of the velocity with which the train moves. In all mathematical curves the ordinate of any point is a function of its abscissa, that is, $y$ is a function of $x$, where $y$ represents the ordinate and $x$ the abscissa. The mathematical way of expressing this fact is $y=f(x)$ which is read " $y$ equals $f$ of $x$ " and means " $y$ is a function of $x . "$

Every equation between $x$ and $y$ when solved for $y$ in terms of $x$ defines a function of $x$, when solved for $x$ in terms of $y$ defines a function of $y$. Suppose we have solved the equation for $y$ in terms of $x$, we then have $y=f(x)$ and by using values of $x$ for abscissas and the corresponding values of $y$ for ordinate a curve may be constructed which represents the function graphically. Speaking more precisely, the ordinates of the curve thus obtained represent the values of the function for corresponding values of the abscissas.

13.2. Maximum and Minimum Values of a Function. Let $y=f(x)$ be some given function of $x$ and let figure 18 represent its graph. As $x$ increases beginning with $0, y$ decreases; from $P_{1}$ to $P_{2} y$ increases; from $P_{2}$ to $P_{3} y$ decreases again, 
etc. The points $P_{1}, P_{2}, P_{3}, P_{4}$, etc. at which $y$ changes from a decreasing function to an increasing function or vice versa, are called turning points of the function.

A turning point at which the function changes from a decreasing function to an increasing function, as $P_{1}, P_{3}$, or $P_{5}$, is

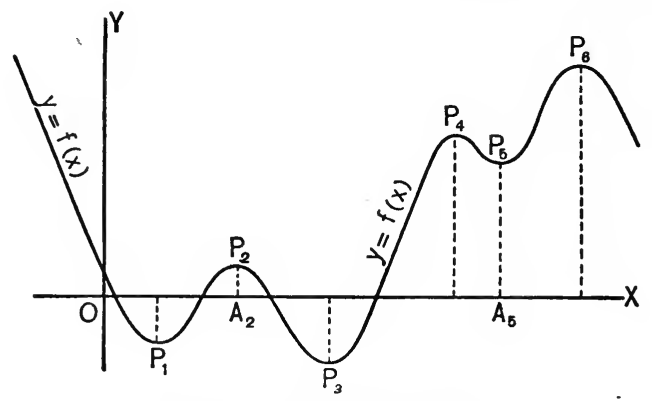

Fia. 18. - Turning Points.

called a minimum; a turning point at which the function changes from an increasing function to a decreasing function, as $P_{2}, P_{4}$, or $P_{6}$, is called a maximum. At each of the points $P_{1}, P_{3}, P_{5}$, the function $y$ has a minimum value, at each of the points $P_{2}, P_{4}, P_{6}$, a maximum value.

Plainly, a function may have two or more maxima or minima or it may have neither a maximum nor a minimum. Nor is a maximum value necessarily the greatest value a function may have or a minimum the least value. A minimum value may in fact be greater than a maximum, value as for instance the value $A_{5} P_{5}$ in figure 18, which is greater than the value $A_{2} P_{2}$.

13.3. Test for a Maximum or Minimum. It follows from what has been said that the ordinate of a curve represents a maximum value of the function if it is greater than the ordinates that immediately precede or follow it, and the ordinate represents a minimum value if it is less than the ordinates 
that immediately precede or follow it. This consideration leads to the following

Test: The function $y=f(x)$ has the maximum value $y_{\boldsymbol{M}}$ at $x=a$ if, provided $h$ is sufficiently small,

$$
f(a-h)<f(a)>f(a+h) .
$$

Similarly the function $y=f(x)$ has a minimum value $y_{m}$ at $x=b$ if, provided $h$ is sufficiently small,

$$
f(b-h)>f(b)<f(b+h) .
$$

\subsection{Problems Involving Maxima and Minima. The} method of representing functions by graphs enables us to obtain approximate solutions of a great variety of problems whose exact solutions require a knowledge of the differential and integral calculus. We shall consider some typical problems of this kind.

Problem 1. Maximum. A rectangular stockade is to be constructed on the bank of a river. If $\mathbf{1 0 0 0}$ feet of wire fencing are available for three sides of the stockade, the fourth side being formed by the river, what must be the dimensions of the stockade to inclose the largest area possible?

Solution. Let $y$ represent the area, $x$ the length, and $w$ the width of the stockade. The area of the stockade is $y=x w$ and from the condition of the problem $x+2 w=1000$, from which $w=(1000-x) / 2$. Substituting this value of $w$ in $y=x w$ we have

$$
y=\frac{x(1000-x)}{2} .
$$

Our problem now is to find the value of $x$ which will make $y$ a maximum. To find this value we plot the curve of $y=x(100-x) / 2$. The abscissa of the highest point on this curve will give us the required value of $x$, the ordinate of this same point gives us the maximum area. The values of $x$ and $y$ used in plotting the curve are given in the table below and the curve itself is shown in figure 19. 


\begin{tabular}{r|r}
\multicolumn{1}{c|}{$x$} & \multicolumn{1}{c}{$y$} \\
\cline { 2 - 2 } 100 & 45000 \\
200 & 80000 \\
300 & 105000 \\
400 & 120000 \\
500 & 125000 \\
600 & 120000 \\
700 & 105000 \\
800 & 80000 \\
900 & 45000 \\
1000 & 0 \\
\hline
\end{tabular}

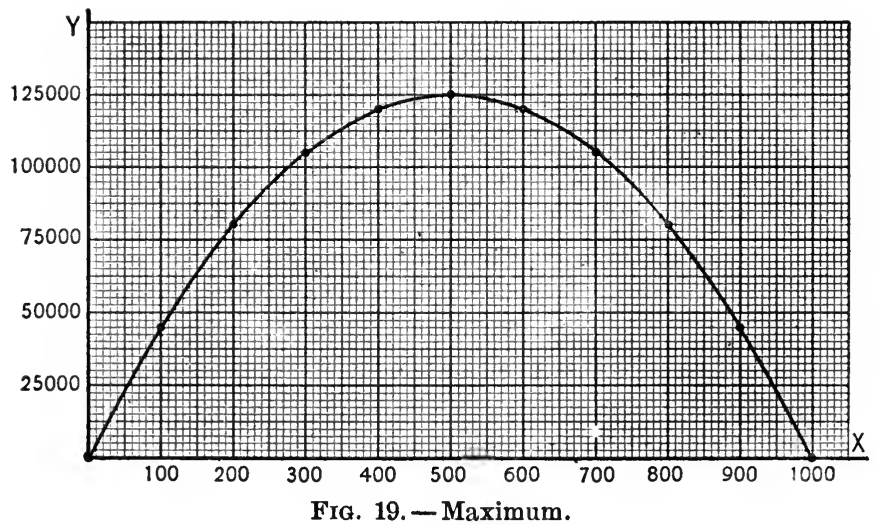

The curve shows at a glance that $y$ has the greatest value when $x=$ 500 , that is, the largest possible stockade that can be built under the given conditions by using 1000 feet of fencing is 500 feet long and consequently 250 feet wide and the largest area inclosed is 125,000 square feet.

We may check our result by employing the test in 13.3. The ordinates for abscissas a little less as well as a little greater than 5000 must be less than 125,000 if 125,000 is the maximum value of the function. Substituting in the equation $x=499$ we find $y=124,999.5$ and substituting $x=501$ we find $y=124,999.5$. Both of these ordinates are less than 125,000 , as they should be. 
Problem 2. Minimum. $A$ and $B$ (fig. 20) are two army posts stationed at distances $A C=5$ miles and $B D=10$ miles from a river $C D$ The distance between $C$ and $D$, the nearest points on the river, is 20 miles. A pumping station, $P$, on the river is to supply both posts with water. How far from $C$ must the pumping station be located so as to make the combined lengths of pipe lines from $P$ to $A$ and $B$ the shortest possible?

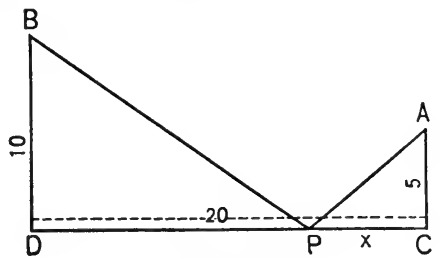

Fig. 20.

Solution. Let $x$ denote the distance of $P$ from $C$ and $y$ the combined length of the pipe lines so that $y=A P+P B$. Since $C D=20, P D=20$ $-x$, and we now have from the right triangles $A C P$ and $P D B$, $A P^{2}=x^{2}+5^{2}=x^{2}+25, \quad P B^{2}=(20-x)^{2}+10^{2}=(20-x)^{2}+100$, and therefore

$$
y=\sqrt{x^{2}+25}+\sqrt{(20-x)^{2}+100} .
$$

Our problem is to discover what value of $x$ will make this function a minimum.

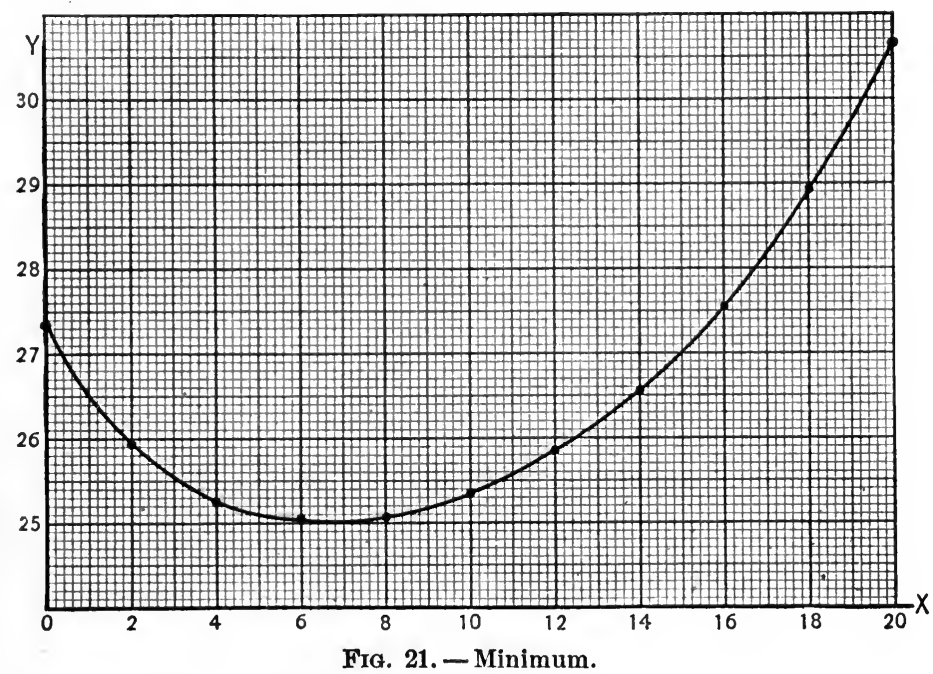

We next plot the curve of the equation just obtained. The computation work is facilitated by arranging the work in tabular form as shown below. 


\begin{tabular}{|c|c|c|c|c|c|c|c|c|c|c|c|c|}
\hline $\boldsymbol{x}$ & $=$ & 0 & 2 & 4 & 6 & 8 & 10 & 12 & 14 & 16 & 18 & 20 \\
\hline$x^{2}+5$ & $=$ & 25 & 29 & 41 & 61 & 89 & 125 & 169 & 221 & 281 & 349 & 425 \\
\hline$(20-x)^{2}+100$ & $=$ & 500 & 424 & 356 & 296 & 244 & 200 & 164 & 136 & 116 & 104 & 100 \\
\hline$\sqrt{x^{2}+5}$ & $=$ & 5 & 5.385 & 6.403 & 7.810 & 9.4341 & 1.180 & 13.000 & 14.866 & 16.763 & 18.682 & 20.616 \\
\hline$\sqrt{(20-x)^{2}+11}$ & $=2$ & .361 & 20.591 & 18.568 & 17.205 & 15.620 & 14.142 & 12.806 & 11.662 & 10.770 & 10.198 & 10.000 \\
\hline$y$ & $=2$ & & 25.98 & 25.27 & 25.02 & 25.05 & 25.32 & 25.81 & 26.53 & 27.54 & 28.88 & 30.62 \\
\hline
\end{tabular}

An inspection of the graph (fig. 21) shows that there is a minimum value of $y$ somewhere between $x=6$ and $x=8$. To determine the value

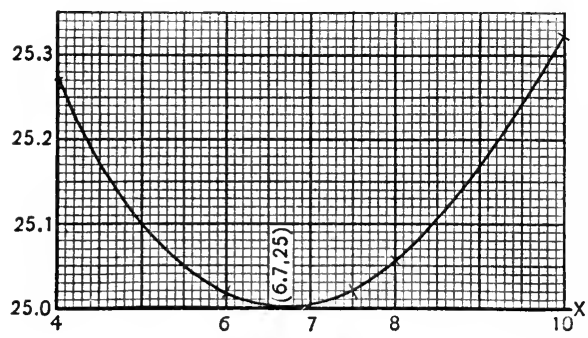

Fra. 22. of $x$ more accurately it is best to redraw the portion of the graph in which the minimum occurs on an enlarged scale as in figure 22. In drawing this portion of the graph we have first plotted several additional points, namely the points $(6.5,25.006),(7.0,25.003)$, $(7.5,25.023)$. It is now easily seen that the graph has a minimum value at $x=6.7$ and that for this value of $x$ the value of $y$ is 25 .

Therefore the pumping station should be located 6.7 miles from $C$ and the shortest possible combined length of the pipe lines is 25 miles.

Note. The exact value of $x$ as given by differential calculus is $6 \frac{2}{3}$ miles.

13.5. Area under a Curve. By the area under a curve is meant the area bounded by the curve, two ordinates, and a portion of the $x$-axis, as the area $P_{1} P_{7} Q_{i} Q_{1}$, in figure 23. Such an area may be computed approximately as follows: We divide the base $Q_{1} Q_{7}$ into equal parts of length $h$. If at the points of division ordinates are erected meeting the curve in the points $P_{2}, P_{3}, P_{4}$, etc., the area to Fra. 23. - Area under a be found is divided into strips of equal

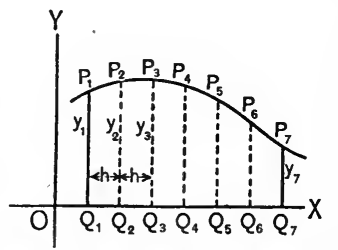
widths $h$, and trapezoidal in shape. Let us denote the ordinates of the points $P_{1}, P_{2}, P_{3}$, by $y_{1}, y_{2}, y_{3}$, etc. If $P_{1} P_{2}$ were 
a straight line the area of the strip $Q_{1} P_{1} P_{2} Q_{2}$ would be exactly equal to $\frac{1}{2} h\left(y_{1}+y_{2}\right)$ the area of the strip $Q_{2} P_{2} P_{3} Q_{3}$ equal to $\frac{1}{2} h\left(y_{2}+y_{3}\right)$, and so on for each of the other strips. Then the total area is equal to the sum of these or $\frac{1}{2} h\left(y_{1}+y_{2}\right)+\frac{1}{2} h\left(y_{2}+y_{3}\right)$ $+\frac{1}{2} h\left(y_{3}+y_{4}\right)+$ etc., which when simplified gives us the socalled trapezoidal rule for the area under a curve, namely

Area of $Q_{1} P_{1} P_{7} Q_{7}=\frac{1}{2} h\left(y_{1}+2 y_{2}+2 y_{3}+\cdots+2 y_{6}+y_{7}\right)$.

Of course $P_{1} P_{2}$ is not a straight line but a curve, so that the trapezoidal rule will not give the exact area under the curve. The greater the number of strips into which the area is divided the more accurate will be the result.

A more accurate rule is Simpson's one-third rule, which is: Divide the area into an even number of strips of equal widths so that there are an odd number of ordinates; then, approximately, Area of $Q_{1} P_{1} P_{n} Q_{n}=$

$$
\frac{h}{3}\left(y_{1}+4 y_{2}+2 y_{3}+4 y_{4}+2 y_{5}+\cdots+2 y_{n-2}+4 y_{n-1}+y_{n}\right) .
$$

The proof of this rule is too difficult to be given here.

If the boundary curve is given by its equation the length of the ordinates may be computed, otherwise they must be measured. The trapezoidal rule is commonly used by surveyors in computing the acreage of fields bounded on one side by a river or curve.

\subsection{Area of a Closed} Curve. A very simple and practical method of finding the area of a closed curve is to lay out the curve on coördinate paper and then count the num-

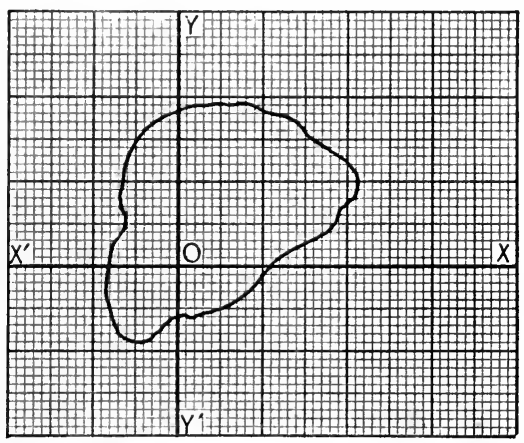

Fig. 24. - Area of Closed Curve. 
ber of little squares which it covers, allowing for fractional squares as well. The total area of the curve is then easily found by multiplying the area of one of the little squares by the number of squares. This method has the advantage that it can be applied to irregular curves as well as to mathematical curves. If each of the larger squares in figure 24 represents a square inch the area of the figure will be found to be 5.185 sq. in.

\section{EXERCISE 13}

1. Show that the function $y=1 /\left(1+x^{2}\right)$ has a maximum value for $x=0$.

2. Show that the function $y=(x+1)(x-3)$ has a minimum value for $x=1$.

3. A battleship lies at anchor, at $S, 6$ miles off shore. A detachment of marines is to be sent from the ship to reinforce

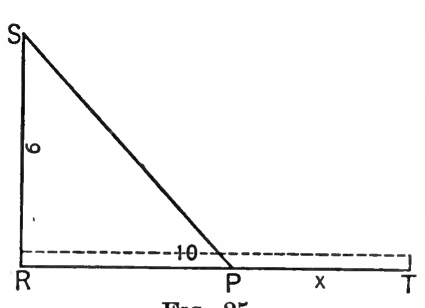

Fra. 25. a body of troops who are engaging the enemy at a point $T, 10$ miles from the nearest point $R$ along the shore. Assuming that the marines can row 2 miles per hour and march at the rate of 3 miles per hour, at what point on the shore must they disembark in order to reach their destination in the shortest time possible?

Ans. At a point 4.63 miles from $T$.

Suggestion. Denote $P T$ by $x$. The time required to reach $T$ is $\frac{S P}{2}+\frac{P T}{3}=y$. Express $S P$ and $P T$ each in terms of $x$ and then find the value of $x$ that will make $y$ a minimum.

4. What are the most economic dimensions of a conicalshaped army tent that will contain $500 \mathrm{cu}$. ft. of air space? 
By the most economic dimensions is meant the dimensions that will require the least canvas.

5. The strength of a rectangular beam is proportional to the product of the width of the cross-section by the square of the depth. Find the dimensions of the strongest beam that can be cut from a $\log 12$ inches in diameter. Ans. $6.93 \times 9.80$.

6. The stiffness of a rectangular beam is proportional to the product of its width by the cube of its depth. Find the dimensions of the stiffest beam that can be cut from a log 12 inches in diameter.

7. A square piece of tin is to be made into an open-top box by cutting out equal squares at the corners and turning up the sides. Find the dimensions of the largest box that can be made from a piece of tin 12 inches square.

Ans. $8 \times 2$.

8. If the power required to propel a steamer in still water is proportional to the cube of the speed, find the most economic rate of steaming against a current of 3 miles per hour.

9. With the center at the point $(2,-1)$ and a radius equal to 3 draw a circle. Compute that portion of the circle which falls in the first quadrant; ( $a$ ) by the trapezoidal rule, $(b)$ by Simpson's one-third rule.

Suggestion. Divide the base line of the required area into 8 equal parts.

Ans. (a) 7.65 .

(b) 7.46 .

10. Figure 26 represents a farm tract bounded on one side by a river. In order to determine its acreage a surveyor runs offsets at intervals of five rods as shown in the figure and measures the length of each offset.

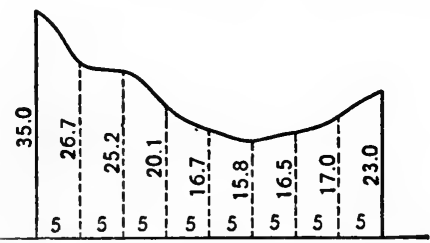

Frg. 26. - Area by Trapezoidal Rule.

All the numbers represent rods. How many acres does the farm tract contain? 
LESSON XIV - THE STRAIGHT LINE AND CIRCLE

14.1. To Find the Equation of a Straight Line. Let $A B$ represent any straight line, $P(x, y)$ any point on it, $R$ the point

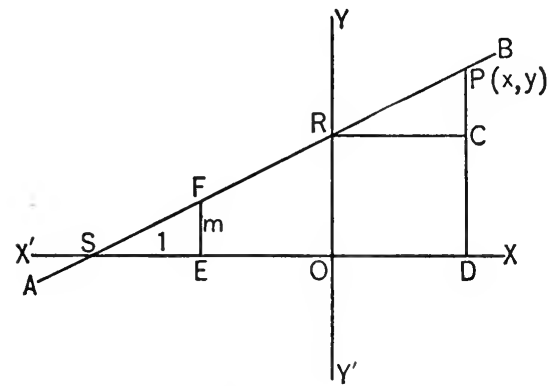

Fig. 27. in which it intersects the $y$-axis, $S$ the point in which it intersects the $x$-axis. Through $P$ draw $P D$ perpendicular to the $x$-axis and through $R$ draw $R C$ parallel to the $x$-axis. At $E$, a unit's distance from $S$, erect the perpendicular $E F$. Denote $O R$ by $b$ and $E F$ by $m$.

The triangles $R C P$ and $S E F$ are similar; we have therefore $\frac{C P}{R C}=\frac{E F}{S E}=\frac{m}{1}$, from which $C P=m \cdot R C$. But $C P=D P-$ $O R=y-b$, and $R C=O D=x$, hence on substituting we obtain $y-b=m x$, or

$$
y=m x+b .
$$

This is the required equation, for it expresses the relation between the coördinates $x$ and $y$ of any point on the line.

$m$, which determines the direction of the line, is called the slope of the line.

$b$, which is the distance cut off by the line on the $y$-axis, is called the $y$-intercept of the line.

All parallel lines have the same value of $m$. 
All lines passing through $R$ have the same value of $b$.

When $m$ is zero the line is parallel to the $x$-axis.

When $b$ is zero the line passes through the origin.

\subsection{Every Equation of the First Degree in $x$ and $y \operatorname{Re}_{-}^{-}$-} resents a Straight Line. Every equation of the first degree in $x$ and $y$ may be written in the form $A x+B y+C=0$, where $A, B$, and $C$ are constants which may have any values, including zero. If we solve this equation for $y$ we obtain $y=-\frac{A}{B} x-\frac{C}{B}$, which is of the form $y=m x+b$, where $m=-\frac{A}{B}$ and $b=-\frac{C}{B}$. This shows that the equation $A x+B y+C=0$ represents a line whose slope is $-A / B$ and whose $y$-intercept is $-C / B$.

Because equations of the first degree in two unknowns may be represented by straight lines such equations are called linear equations.

14.3. To Construct a Straight Line from its Equation. One way is to plot two of its points and then draw the straight line connecting these two points.

Another way is to draw first a line through the origin having the slope $m=-A / B$. We then measure off a point $R$ on the $y$-axis whose distance from the origin is $-C / B$ and then draw a line through this point parallel to the line drawn through the origin This second line is

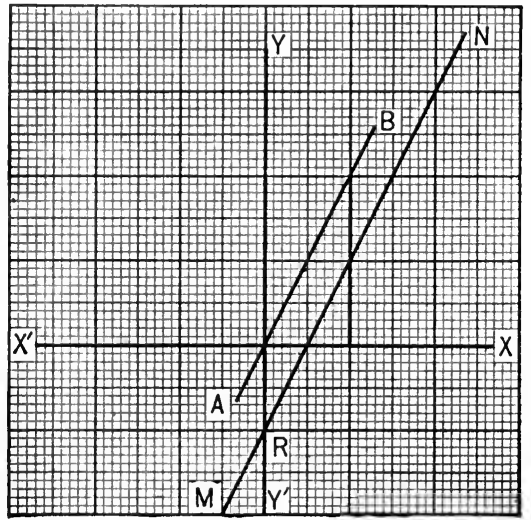

FIG. $28 .-2 x-y-1=0$. 
the required line, for it has the required slope as well as the required $y$-intercept.

Example 1. Construct the line whose equation is $2 x-y-1=0$.

Solution. Solving for $y$ we find $y=2 x-1$, which shows that the slope of the line is 2 and the $y$-intercept is -1 . We next draw a line $A B$ through the origin having the slope 2 (fig. 28), then locate a point

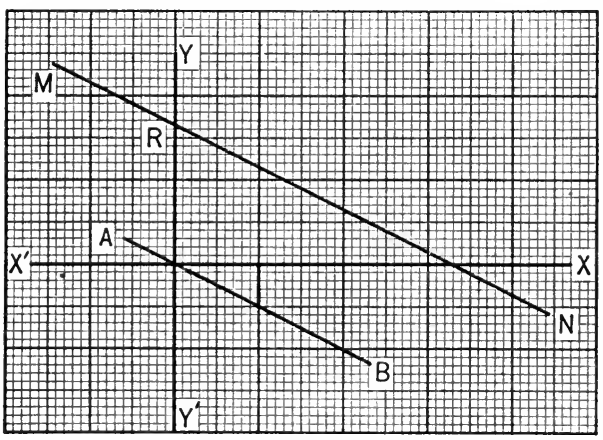

Fig. 29. $-3 x+6 y-10=0$.

$R$ on the $y$-axis whose distance from the origin is -1 , and finally draw a line $M N$ through $R$ parallel to the line $A B$. $M N$ is the required line.

Example 2. Construct the line $3 x+6 y-10=0$.

Solution. Solving for $y$ we find $y=-\frac{1}{2} x+\frac{5}{3}$. This shows that the slope of the line is $-\frac{1}{2}$ and its $y$-intercept is $+\frac{5}{3}$. Proceeding as in example 1 we obtain the line $M N$ (fig. 29).

14.4. To Find the Equation of a Circle. In figure 30 let $C(a, b)$ represent the center of the circle, $r$ its radius, $P(x, y)$ any point on the circumference of the circle. Join $C$ and $P$. Through $P$ draw $P F$ perpendicular to the $x$-axis and through $C$ draw $C E$ parallel to the $x$-axis. Then in the right triangle $C E P$,

$$
\begin{aligned}
(C E)^{2}+ & (E P)^{2} \\
& =(C P)^{2}=r^{2} .
\end{aligned}
$$

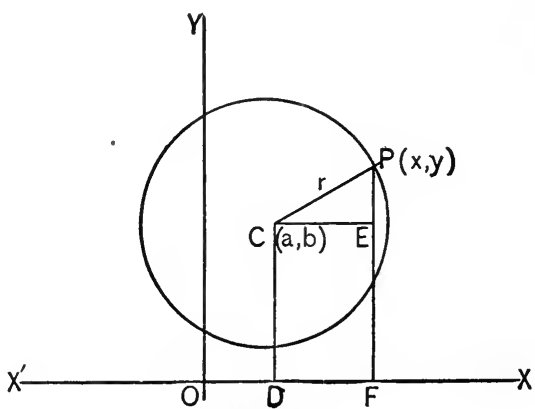

Fig. 30 .

Now $C E=O F-O D=x-a$, and $E P=F P-D C=y-b$. 
therefore

$$
(x-a)^{2}+(y-b)^{2}=r^{2} .
$$

This is the required equation, for it expresses the relation between the coördinates $x$ and $y$ of any point on the circumference of the circle.

If the center of the circle is at the origin, $a$ and $b$ are each equal to zero. In that case the equation of the circle is

$$
x^{2}+y^{2}=r^{2} \text {. }
$$

14.5. Every Equation of the Form $x^{2}+y^{2}+2 G x+2 F y+$ $C=0$ represents a circle. By adding $G^{2}+F^{2}$ to both members of the equation and transposing the constant term $C$ we have

$$
x^{2}+y^{2}+2 G x+2 F y+G^{2}+F^{2}=G^{2}+F^{2}-C,
$$

which may be written

or

$$
\left(x^{2}+2 G x+G^{2}\right)+\left(y^{2}+2 F y+F^{2}\right)=G^{2}+F^{2}-C,
$$

which is of the form $(x-a)^{2}+(y-b)^{2}=r^{2}$, where

$$
(x+G)^{2}+\left(y+F^{\prime}\right)^{2}=G^{2}+F^{2}-C,
$$

This shows that the equation $x^{2}+y^{2}+2 G x+2 F y+C=0$ represents a circle whose center is at the point $\left(-G,-F^{\prime}\right)$ and whose radius is $\sqrt{G^{2}+F^{2}-C}$.

If $G=0$ the center of the circle lies on the $y$-axis, if $F=0$ the center of the circle lies on the $x$-axis, if both $G$ and $F$ are zero the center is at the origin.

If $G^{2}+F^{2}-C$ is negative, $r$ is an imaginary number. In that case, the equation represents an imaginary circle which can not be drawn.

It should be observed that the equation of the circle is an equation of the second degree in which the term in $x y$ is missing and in which the coefficients of $x^{2}$ and $y^{2}$ are equal. If the coefficients of $x^{2}$ and $y^{2}$ are equal but not equal to 1 , we may 
reduce the equation to the required form by dividing it by this coefficient.

\subsection{To Construct a Circle from its Equation. Direc-} tions. Reduce the equation of the circle to the form $(x-a)^{2}+(y-b)^{2}=r^{2}$. Locate the point $(+a,+b)$. With

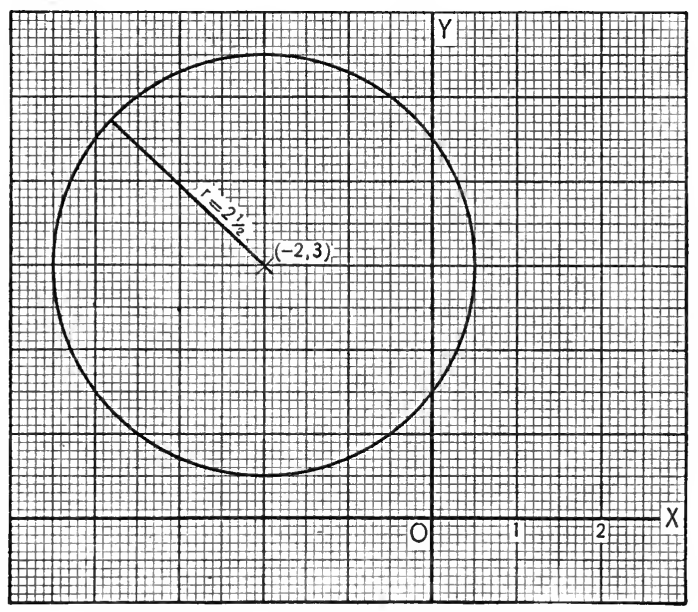

FIG. 31. $-(x+2)^{2}+(y-3)^{2}=\left(\frac{5}{2}\right)^{2}$.

this point as the center and a radius equal to $r$ describe a circle. This will be the required circle.

Example 1. Construct the circle whose equation is

$$
4 x^{2}+16 x+4 y^{2}-24 y+27=0 .
$$

Solution. Dividing the equation by 4 we have

$$
x^{2}+4 x+y^{2}-6 y+\frac{27}{4}=0 .
$$

Completing the squares in $x$ and $y$,

$$
\left(x^{2}+4 x+4\right)+\left(y^{2}-6 y+9\right)=4+9-\frac{27}{4}=\frac{25}{4},
$$

or

$$
(x+2)^{2}+(y-3)^{2}=\left(\frac{5}{2}\right)^{2} .
$$

The required circle has its center at the point $(-2,3)$ and has a radius equal to $\frac{5}{2}$ (fig. 31 ). 
Example 2. Find the equation of the circle whose center is at the point $\left(\frac{2}{3},-\frac{1}{2}\right)$ and whose radius is 10 .

Solution. The equation is

Simplifying,

$$
\left(x-\frac{2}{3}\right)^{2}+\left(y+\frac{1}{2}\right)^{2}=10^{2} .
$$

$$
\begin{aligned}
x^{2}+\frac{x}{3}+\frac{4}{9}+y^{2}+y+\frac{1}{4} & =100, \\
x^{2}-\frac{x}{3}+y^{2}+y & =100-\frac{25}{36}
\end{aligned}
$$

Clearing fractions, $36 x^{2}+36 y^{2}-12 x+36 y=3575$.

\section{EXERCISE 14}

1. On the same sheet draw the five lines:

$y=2 x+2, y=2 x+1, \quad y=2 x, \quad y=2 x-1, \quad y=2 x-2$.

2. On the same sheet of paper draw the four lines:

$y-x=1, \quad y+x=1, \quad y=1, \quad x=0$.

3. Write the equation of the line which cuts the $y$-axis 5 units below the origin and makes an angle of $45^{\circ}$ with the positive direction of the $x$-axis.

4. Show that if two lines are perpendicular the product of their slopes is -1 , that is, if the equations of the two lines are $y=m x+b$, and $y=m^{\prime} x+b^{\prime}$, then $m m^{\prime}=-1$, or $m^{\prime}=-1 / m$.

5. Write and simplify the equations of the following circles:

(a) Center at $(3,4)$, radius 5 ;

(b) Center at $(-3,4)$, radius 4 ;

(c) Center at $(-3,-4)$, radius 1 ;

(d) Center at $(5,0)$, radius 5 ;

(e) Center at $(0,-10)$, radius $\frac{3}{4}$;

(f) Center at origin, radius $r$.

Ans. (a) $\quad x^{2}-6 x+y^{2}-8 y=0$;

(b) $x^{2}+6 x+y^{2}-8 y+9=0$; etc.

6. Determine the coördinates of the center and the length of the radius of each of the following circles : 

(a) $x^{2}+y^{2}=2$;
(d) $x^{2}-3 x-y^{2}=0$;
(b) $x^{2}-4 x+y^{2}-6 y+12=0$;
(e) $4 x^{2}+12 x+4 y^{2}-91=0$;
(c) $x^{2}+2 x+y^{2}+2 y=0$;
(f) $3 x^{2}-5 x+3 y^{2}+6 y=-3$.
Ans. (a) $(0,0), \sqrt{ } 2$;
(b) $(2,3), 1$;
(f) $\left(\frac{5}{6},-1\right), 5 / 6$.

7. Find the vertices of the triangle formed by the three lines

$$
\begin{aligned}
& x+y-4=0, \quad x-2 y+8=0, x+4 y-10=0 . \\
& \text { Ans. }(0,4),(-2,3),(2,2) .
\end{aligned}
$$

8. Find the points in which the straight line $x+y=1$ intersects the circle $x^{2}+10 x+y^{2}+2 y+1=0$.

$$
\text { Ans. (-2, 3), (-1, 2). }
$$

9. Find the intersection points of the two circles $x^{2}+14 x+y^{2}+4 y-47=0, \quad x^{2}-14 x+y^{2}-24 y+93=0$. Ans. (-1, 6), $(1,4)$. 


\section{LESSON XV - GRAPHIC SOLUTION OF EQUATIONS}

15.1. Single Equations in Two Unknowns. We have seen in Lesson XII that every equation between two unknowns, in which one of the unknowns can be expressed in terms of the other, may be represented by some curve, and we learned how to construct this curve by plotting points. The relation between the curve and its equation may be expressed in two statements or principles, -

Principle 1. The coördinates of every point on the curve satisfy the equation of the curve.

Principle 2. The curve passes through every point whose coördinates satisfy the equation of the curve.

15.2. Simultaneous Equations in Two Unknowns. Simultaneous equations are usually defined as equations which are satisfied by the same values of the unknowns. These common values are called the roots of the equations: to solve the equations is to find their roots.

Let $F_{1}=0$, and $F_{2}=0$ denote two simultaneous equations in two unknowns, $C_{1}$ and $C_{2}$ the curves representing $F_{1}=0$ and $F_{2}=0$ respectively referred to the same coördinate axes. By Principle 1 of the preceding section the coördinates of every point on $C_{1}$ satisfy the equation $F_{1}=0$, and the coördinates of every point of $C_{2}$ satisfy the equation $F_{2}=0$, therefore the coördinates of every point common to the two curves must satisfy both equations. These coördinates are therefore a pair of roots of the given equations. We see furthermore that there are as many pairs of real roots of the two equations as there are intersection points of the two curves. 


\subsection{Graphic Solution of Simultaneous Equations in} Two Unknowns. The foregoing considerations lead to the following simple rule for finding the real roots of a pair of simultaneous equations in two variables.

1. Plot the curve of each equation using the same coördinate axes.

2. Measure the coördinates of each point of intersection of the two curves.

3. Each pair of coördinates gives a solution of the two equations.

15.4. Graphic Solution of Linear Equations in Two Unknowns. Let it be required to solve the two equations $A x+$ $B y+C=0$ and $A^{\prime} x+B^{\prime} y+C^{\prime \prime}=0$. The graphs of these equations are straight lines which are readily drawn. If these two lines intersect the equations have a solution, namely, the coördinates of the point of intersection. Since two straight lines can not intersect in more than one point, two such equations can have at most one solution.

If the two straight lines are parallel they do not intersect. In that case the two equations have no common solution. Two linear equations which have no common solution are said to be inconsistent. The problem which gives rise to such equations imposes impossible conditions.

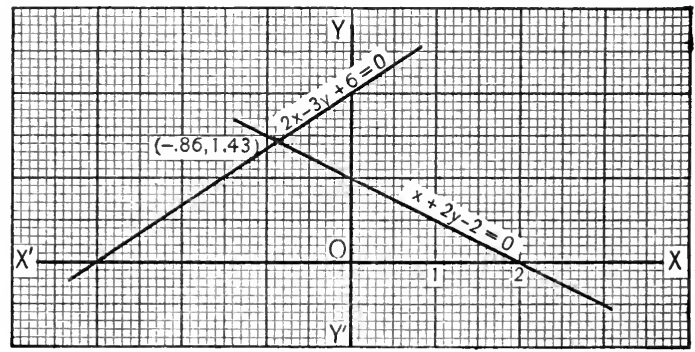

Fig. $32 .-x+2 y-2=0,2 x-3 y+6=0$. 
If the two straight lines coincide the two equations are equivalent. The problem which gives rise to such equations is indefinite and has an infinite number of solutions, that is, every point on either line is common to both lines.

Example. Solve the two equations $x+2 y-2=0,2 x-3 y+6=0$.

Solution. Plot the two straight lines as shown in figure 32 . The coördinates of their intersection point are $x=-0.86 y=1.43$. These are the approximate values of the roots correct to two places of decimals.

15.5. Graphic Solution of Simultaneous Equations of Higher Degrees. The graphic method of solving equations is of special advantage when the methods of elementary algebra fail. Such equations as $x-y-1=0, x^{2}-y^{2}-5=0$, can easily be solved algebraically, and it would therefore be only a waste of time to apply the graphic method in such cases. But there are other equations which can not be solved by elementary algebra.

In such cases the graphic method is the only method available to the beginner. Suppose it were required to solve the equations

$$
x^{2}+y=7, x+y^{2}=11 \text {. }
$$

These equations look simple enough, but even the best student in elementary algebra will fail to find all the solu-

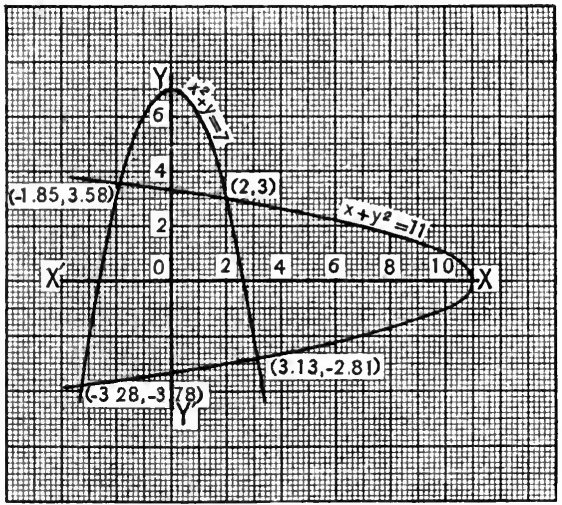

Fig. 33. $-x^{2}+y=7, x+y^{2}=11$. tions by algebra. We shall solve these equations by the graphic method.

Example. To find all the roots of the two equations

$$
x^{2}+y=7, \quad x+y^{2}=11 \text {. }
$$


Solution. Plot the two curves as shown in figure 33. The four points of intersection are

$$
\begin{aligned}
& P_{1}=(2,3), P_{2}=(-1.85,3.58), \\
& P_{3}=(-3.28,-3.78), P_{4}=(3.13,-2.81) .
\end{aligned}
$$

The roots of the equations are therefore

$$
\begin{array}{rrrr}
x=2, & -1.85, & -3.28, & 3.13, \\
y=3, & 3.58, & -3.78, & -2.81 .
\end{array}
$$

These results are correct to two places of decimals.

15.6. Graphic Solution of the Quadratic Equation $a x^{2}+b x$ $+c=0$. Consider the simultaneous equations

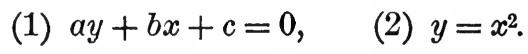

If we substitute the value of $y$ from (2) in (1) we obtain

$$
\text { (3) } a x^{2}+b x+c=0 \text {. }
$$

This shows that the values of $x$ for points which are common to the two curves (1) and (2) also satisfy equation (3). We have therefore the following simple rule for finding the real roots of the quadratic (3): Construct the straight line (1) and the curve (2) using the same axes of coördinates. The abscissas of the points of intersection of the straight line (1) with the curve (2) are the roots of equation (3).

The curve $y=x^{2}$ which we have used repeatedly is called the unit parabola. Since it does not depend on the particular values of $a, b$, and $c$, it is the same for all quadratic equations. When this parabola has once been carefully constructed, it can be used for solving any number of quadratic equations. All that remains to be done to solve any given quadratic $a x^{2}$ $+b x+c=0$ is to draw the straight line $a y+b x+c=0$ and to read off the abscissas of the points in which this line intersects the parabola.

Example. Find the real roots of the quadratics
(a) $x^{2}-x-1=0$,
(c) $5 x^{2}-4 x-10=0$,
(e) $x^{2}+x+1=0$
(b) $3 x^{2}+2 x-2=0$,
(d) $x^{2}-2 x+1=0$, 
Solution. Construct the parabola $y=x^{2}$ and also the five straight lines (a) $y-x-1=0$, (b) $3 y+2 x-2=0$, (c) $5 y-4 x-10=0, \quad(d)$ $y-2 x+1=0$, (e) $y+x+1=0$. The abscissas of the intersection points of these lines with the parabola are the required roots, viz.,

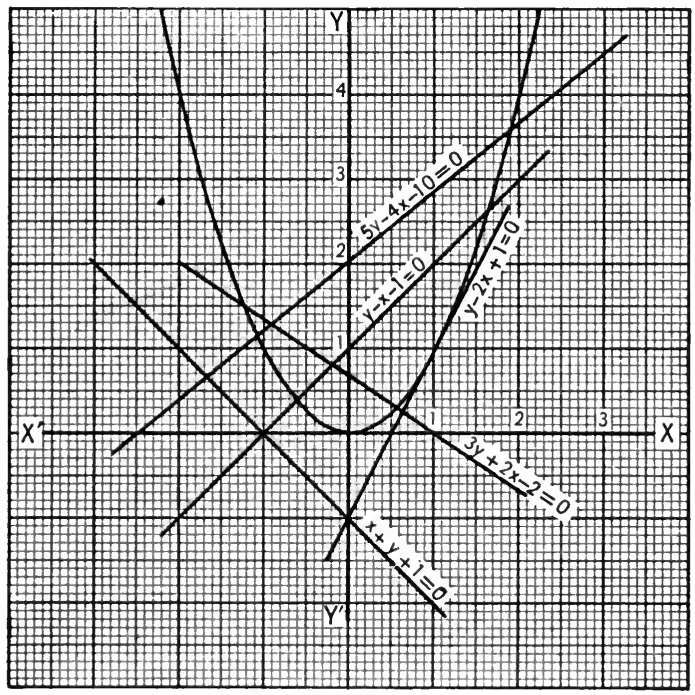

Fig. 34 .
(a) $1.62,-0.62$;
(b) $0.55,-1.22$;
(c) $1.87,-1.07$
(d) 1,1 ;

(e) imaginary.

It will be observed that the line $(e)$ does not intersect the parabola. This means that the corresponding quadratic equation has no real roots. Furthermore it will be seen that the line $(d)$ is tangent to the parabola. This means that the corresponding quadratic has equal roots.

\subsection{Graphic Solution of the Cubic Equation $a x^{3}+c x+d$} $=0$. Consider the simultaneous equations

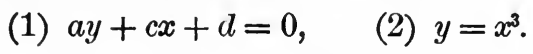

If in (1) we substitute the value of $y$ from (2) we obtain

$$
\text { (3) } a x^{3}+c x+d=0 \text {. }
$$

Reasoning as in 15.6 we see that the roots of (3) are the ab- 
scissas of the intersection points of the straight line (1) with the curve (2). The curve $y=x^{3}$ used in this solution is called the cubical parabola and since it is independent of the constants $a, c$, and $d$, it is clear that the same cubical parabola will answer for the solution of any cubic of the form (3).

Example. Solve the cubic equation $3 x^{3}+x-11=0$.

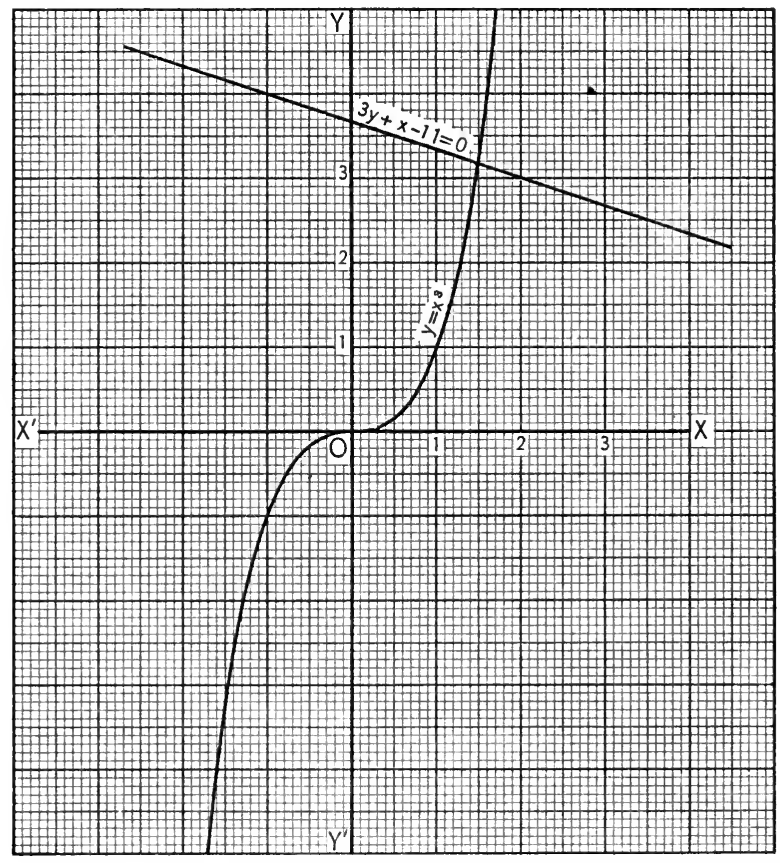

FIG. $35 .-3 x^{3}+x-11=0$.

Solution. Construct the cubical parabola $y=x^{3}$ and also the straight line $3 y+x-11=0$ (fig. 35). The abscissa of the point in which the two curves intersect is $x=1.48$. This is the required root.

Since the straight line intersects the cubical parabola in only one point, the equation $3 x^{3}+x-11=0$ has only one real root, the other two roots being imaginary. 
15.8. Graphic Solution of the General Cubic $A X^{3}+B X^{2}$ $+C X+D=0$. In this equation put $X=x=\frac{B}{3 A}$, the equation then takes the form $a x^{3}+c x+d=0$ from which $x$ may be found as in 15.7 and from this $X$.

Example. Solve the equation $375 X^{3}-450 X^{2}+305 X-1449=0$.

Solution. Here $A=375, B=-450$, hence $B /(3 A)=-450 /(3 \times 375)$ $=-\frac{2}{5}$. Put $X=x-B /(3 A)=x+\frac{2}{5}$, then $375 X^{3}-450 X^{2}+305 X$ $-1449=375\left(x+\frac{2}{5}\right)^{3}-450\left(x+\frac{2}{5}\right)^{2}+305\left(x+\frac{2}{5}\right)-1449=375 x^{3}+125 x$ $-1375=0$, or dividing out the factor $125,3 x^{3}+x-11=0$. In 15.7 the real root of this equation was found to be $x=1.48$, hence the real root of the equation $375 X^{3}-450 X^{2}+305 X-1449=0$ is $X=x+\frac{2}{5}$ $=1.48+0.4=1.88$.

15.9. Graphic Solution of the Quartic Equation $a x^{4}+c x^{2}$ $+d x+e=0$. Consider the two simultaneous equations

(1) $a x^{2}+a y^{2}+d x+(c-a) y+e=0, \quad$ (2) $y=x^{2}$.

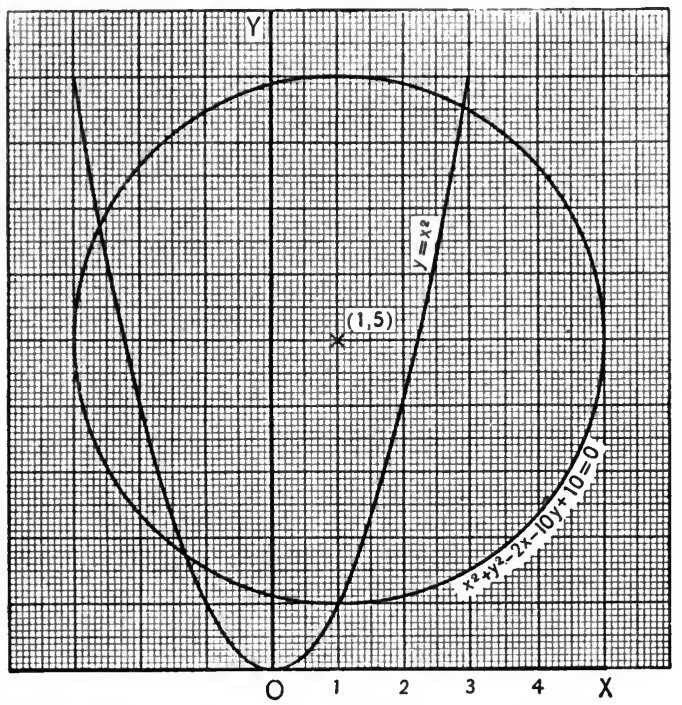

Fig. 36. $-x^{2}+y^{2}-2 x-10 y+10=0, y=x^{2}$. 
If we substitute the value of $y$ from (2) in (1) we obtain (3) $a x^{2}+a x^{4}+d x+(c-a) x^{2}+e=a x^{4}+c x^{2}+d x+e=0$, from which it appears that the roots of the given equation are the abscissas of the intersection points of the two curves (1) and (2). Now (1) is a circle whose radius and the coördinates of whose center may be readily computed by 14.5, and (2) is the unit parabola. This parabola having once been constructed, every quartic of the form (3) may then be solved by drawing a single circle.

Example. Find the roots of the quartic $x^{4}-9 x^{2}-2 x+10=0$.

Solution. Here $a=1, c=-9, d=-2, e=10$, hence
(1) $x^{2}+y^{2}-2 x-10 y+10=0$,
(2) $y=x^{2}$.

By 14.5 (1) is a circle whose radius is 4 and the coördinates of whose center are 1,5. By constructing this circle and the parabola (2) as in figure 36 we find that the circle intersects the parabola in the four points whose abscissas are $-2.60,-1.32,1,2.92$. These numbers are, therefore, the four roots of the given equation.

\subsection{Graphic Solution of the General Quartic Equation} $A X^{4}+B X^{3}+C X^{2}+D X+E=0$.

Put $X=x-\frac{B}{4 A}$, the equation then takes the form $a x^{4}+c x^{2}+d x+e=0$ which may be solved by the method of 15.9 .

Example. Solve the equation

$$
81 X^{4}+216 X^{3}-513 X^{2}-1038 X+394=0 .
$$

Solution. Here $A=81, B=216$, hence $B /(4 A)=216 /(4 \times 81)=\frac{2}{3}$. Substituting $X=x-\frac{2}{3}$ in the above equation we obtain the new equation $81 x^{4}-729 x^{2}-162 x+810=0$, or, dividing through by $81, x^{4}-9 x^{2}$ $-2 x+10=0$. The roots of this equation were found in $\mathbf{1 5 . 9}$ to be $-2.60,-1.32,1$, and 2.92 .

Since $X=x-\frac{2}{3}$ the roots of the given equation are found by diminishing these values by $\frac{2}{3}=0.67$, which gives us the four roots of the given equation $X=-3.27,-1.99,0.33,2.25$. 
15.11. Equivalent Sets of Simultaneous Equations. Two sets of simultaneous equations are equivalent if they have the same roots. It follows from what has been said that the two sets of curves corresponding to the two sets of equations must have the same intersection points. Thus by first adding and then subtracting the two equations
(1) $x^{2}+3 x y+y^{2}=299$,
(2) $x^{2}-3 x y+y^{2}=-1$,

we obtain the equivalent set of equations
(3) $x^{2}+y^{2}=149$,
(4) $x y=50$.

The intersection points of the curves (1) and (2) must coincide with the intersection points of the curves (3) and (4). But these latter curves are much easier to construct than the former.

15.12. General Remarks. In applying the foregoing principles of graphic solution of equations to special cases the student will meet many obstacles which can not be discussed in detail in this place. Some of these obstacles he should be able to overcome without further aid. If further assistance is needed, the student should consult the admirable little text on Graphic Algebra by Professor Arthur Schultze.*

\section{EXERCISE 15}

1. Find the coördinates of the triangle determined by the three lines

$$
\begin{aligned}
23 x+24 y=82.8, & 65 x+17 y=0.2, \quad 42 x-7 y=34.3 \\
& \text { Ans. }(-1.2,4.6),(0.5,-1.9),(1.2,2.3)
\end{aligned}
$$

2. Show graphically that the two sets of equations

$$
\left(\begin{array}{l}
x+2 y=7 \\
2 x-y=4
\end{array}\right) \text { and }\left(\begin{array}{l}
3 x+y=11 \\
x-3 y=-3
\end{array}\right) \text { are equivalent. }
$$

* The Macmillan Co., Publishers, New York. 
3. Find the real roots of the two equations,

$$
y^{2}-3 x+2=0 \text {, and } x^{2}-y-2=0 .
$$

4. Solve the quadratic equation $3 x^{2}-4 x-3 c=0$ for all integral values of $c$ from 1 to 10 . (Approximate roots to the nearest tenth.) Ans. $1.9,-0.5 ; 2.2,-0.9 ; \ldots ; 3.9,-2.6$.

5. A bin is $2 \mathrm{ft}$. wider than it is deep and $2 \mathrm{ft}$. longer than it is wide. Its volume is $130 \mathrm{cu}$. ft. Find its dimensions. (Suggestion. Call the width of the bin $x$.) Ans. $x=5 \frac{1}{3} \mathrm{ft}$.

6. A solid in the shape of a frustum of a cone, altitude 10 in., radius of lower base 10 in., radius of upper base 5 in., is to be cut into two equal parts by a section parallel to a base.

- How far from the upper base must the solid be cut?

Ans. 6.51 in.

7. Find all the real roots of the quartic $y^{4}-22 y^{2}+y+114$ $=0$.

Ans. 3, 3.58, $-3.78,-2.80$.

8. A mine which was purchased for $\$ 100,000$ yielded $\$ 40,000$ net for each of the four years when it was run out. What rate of interest was realized on the investment?

Suggestion. Let $X=1+i$, where $i$ is the rate of interest. The equation is then readily seen to be $100,000 X^{4}-40,000$ $\left(X^{3}+X^{2}+X+1\right)=0$ or $10 X^{4}-4 X^{3}-4 X^{2}-4 X-4=0$. Put $X=x+\frac{1}{10}$, then the equation reduces to the form $x^{4}-$ $0.46 x^{2}-0.488 x-0.4443=0$. By the method of $15.9 x$ is readily found to be 1.12 , hence $X=1.12+0.1=1.22$, and $i=0.22$ or $22 \%$. 


\section{LESSON XVI - REVIEW}

1. What is meant by the coördinates of a point? By the coördinate axes? Find the coördinates of a point midway between the two points $(-4,5)$ and $(3,-2)$. Name some practical applications of coördinate systems.

2. Does a graph give any information which the sets of numbers used in its construction do not contain? What are some of the advantages of the graph over the numbers which give rise to it? What is meant by graphical interpolation? By graphical extrapolation?

3. Construct a graph which may be used for converting mils into minutes and conversely.

4. Construct a graph which may be used both as a table of squares of numbers from 1 to 10 and of square-roots of numbers from 1 to 100 .

5. Construct a graph which may be used to find the reciprocals of all numbers from 1 to 100 .

6. By the graphic method solve problem 7 in exercise 11 .

7. What is a mathematical curve? What is meant by the equation of a curve? Outline the successive steps in plotting a curve from its equation.

8. Plot a curve representing Boyle's Law. (Boyle's Law is expressed by the equation $p v=c$, where $p$ is the pressure exerted by a gas, $v$ its volume, and $c$ some constant. Assume $c=10$.)

9. What is meant by a function of $x$ ? Give some examples. What is meant by a maximum value of a function? By a minimum value? Explain how a maximum or mini- 
mum value of a function may be discovered graphically. Give an absolute test for a maximum. For a minimum.

10. The United States postal regulations require that the combined length and girth of any parcel sent by parcel post shall not exceed 84 inches. Find the dimensions of the largest cylindrical parcel that may be sent by parcel post.

11. The average velocity in miles per hour of a river is given by the formula $v=a+b x+c x^{2}$, where $a, b$, c, are constants and $x$ the depth below the surface of the river, the total depth of the river being taken as 1 . In measurements on the Mississippi river near New Orleans $a, b$, and $c$ were found to be $3.75,0.50$, and -0.75 respectively. Plot a curve showing the velocity of the Mississippi at any depth and find the depth at which the velocity is a maximum.

Ans. The greatest velocity is 3.83 miles per hour at a depth of 0.33 .

12. State in words the Trapezoidal Rule for finding the area under a curve. State in words Simpson's one-third Rule. Give a method for approximating the area of any kind of figure.

13. Prove that the equation $y=m x+b$ represents a straight line and interpret the meanings of $m$ and $b$.

14. What does the equation $(x-a)^{2}+(y-b)^{2}=r^{2}$ represent? Prove it.

15. What is meant by an imaginary circle?.Interpret the equation $x^{2}+y^{2}=0$.

16. Find the center and radius of the circle $3 x^{2}+6 x+3 y^{2}$ $-5 y-1=0$.

17. Find the equation of the circle whose center is at $(0,5)$ and whose radius is 5. Show from the equation that this circle passes through the origin. 
18. If (1) $f(x, y)=0$, and (2) $\phi(x, y)=0$, are two equations whose graphs are $C_{1}$ and $C_{2}$ respectively, show that the graph of every equation of the form $f(x, y)+k \cdot \phi(x, y)=0, k$ being any constant, passes through all the intersection points of $C_{1}$ and $C_{2}$.

19. What is meant by a linear equation? When are two linear equations inconsistent?

20. Show how the real roots of every quadratic equation $a x^{2}+b x+c=0$ can be found graphically by means of the unit parabola and some straight line.

21. Show how the real roots of every cubic equation in $x$ can be found graphically by means of the cubical parabola $y=x^{3}$ and some straight line.

22. Show how the real roots of every quartic equation in $x$ can be found graphically by means of the unit parabola and some circle.

23. When are two sets of simultaneous equations said to be equivalent? Interpret this condition graphically.

24. Is the graphic method of solving equations of any practical value? Illustrate by an example. 


\section{CHAPTER III}

\section{TRIGONOMETRIC FUNCTIONS}

\section{LESSON XVII-THE GENERAL ANGLE AND ITS MEASURES}

17.1. Definition of an Angle. In elementary geometry an angle is frequently defined as the difference in direction, or the amount of opening, between two lines which meet, or tend to meet, in a point. According to this definition no angle can be greater than a straight angle, for obviously the difference in the direction between two lines is greatest when they extend in opposite directions.

For the purposes of higher mathematics it is convenient to think of an angle as formed by rotating a line in a plane about a fixed point and to measure its magnitude by the amount of rotation which the line has undergone in forming the angle. If the rotation is in the clockwise direction the angle is considered negative, if in the counter-clockwise direction, positive. Since there is no limit to the amount of rotation which a line may undergo, it is plain that an angle as now defined may have any magnitude,

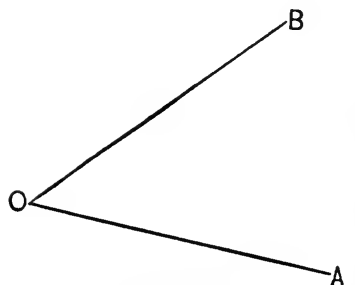

FIG. 37. negative as well as positive.

If the angle in figure 37 has been generated by rotating $O A$ into the position $O B$, it is considered positive and is read "angle $A O B$ "; in this case $O A$ is called the initial side, $O B$ the terminal side. If on the other hand the angle 
has been generated by rotating $O B$ into the position $O A$, the angle is negative and is read "angle $B O A$ "; in that case $O B$ is the initial side and $O A$ the terminal side. Sometimes the sense $(+$ or -$)$ of an angle is indicated by an arrow pointing in the direction in which the angle has been generated.

17.2. Complement and Supplement. If the algebraic sum of two angles is equal to a right angle, each angle is said to be the complement of the other; if their algebraic sum equals two right angles, each is said to be the supplement of the other. That is to say,

if $A+B=90^{\circ}$, then $A$ and $B$ are complementary angles, and if $A+B=180^{\circ}$, then $A$ and $B$ are supplementary angles.

Thus since $100^{\circ}+\left(-10^{\circ}\right)=90^{\circ}$, and $100^{\circ}$ and $-10^{\circ}$ are complementary angles, and since $195^{\circ}+\left(-15^{\circ}\right)=180^{\circ}, 195^{\circ}$ and $-15^{\circ}$ are supplementary angles.

17.3. The Sexagesimal System of Angular Measure. This is the system of angular measure in common use. The principal unit is the degree $\left(^{\circ}\right)$ which is the ninetieth part of a right angle, the secondary units are the minute (') which is the sixtieth part of one degree, and the second (") which is the sixtieth part of one minute. This system dates from the remotest antiquity. It probably originated with the Babylonians who supposed the year to consist of 360 days. On this supposition one degree would measure the average apparent daily motion of the sun among the stars.

17.4. The Decimal System. Instead of subdividing the degree into minutes and seconds, the alternative of subdividing it decimally into tenths, hundredths, and thousandths has been employed. This system has been used more or less ever since the invention of decimal fractions in the sixteenth century. It is taught at the present time alongside the sexagesimal system in a number of American schools and colleges. 
17.5. The Centesimal System. This system which was introduced as a part of the metric system of measures by the French reformers at the time of the French Revolution is widely used in Continental Europe. The principal unit is the hundredth part of a right angle, and is called a grade $(g)$. Each grade is subdivided into one hundred minutes ('), and each minute into one hundred seconds (").

17.6. The Natural or Circular System. Instead of using some arbitrary part of a right angle (as the ninetieth or hundredth) as the unit of angular measure, we may use as the unit an angle, which when placed with its vertex at the center of a circle, subtends an arc equal in length to the radius of

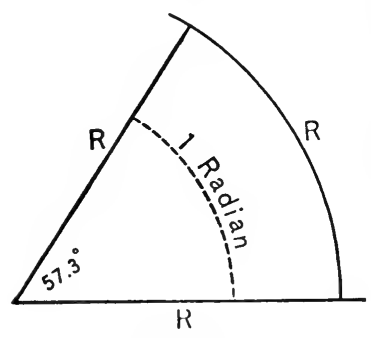

Fig. 38. the circle. This unit is called the radian (fig. 38). The radian measure of any angle is then the abstract number obtained by dividing the length of the arc which the angle intercepts on the circle by the radius of the circle. This system of measuring angles is called the natural system. It is the most convenient measure for many practical purposes and it is commonly used in theoretical investigations.

In military parlance the one-thousandth part of a radian is called a mil. Practically this is the' angle subtended by any unit at a distance of one thousand times that unit, as for instance, the angle subtended by one yard at a distance of one thousand yards. The mil is not commensurable with the complete circle, being contained in it exactly $2000 \pi$, or approximately $6283.18^{+}$, times. For convenience in graduation the complete circle is divided into 6400 parts assumed to be mils, but differing from the true mil by nearly 2 per cent, 
which crror enters into all artillery computations and is neglected.*

\subsection{Comparison of Sexagesimal and Radian Measure.}

To find the relation between the two kinds of units, degrees and radians, it is best to compare the two measures for the entire angular space about a point, that is of four right angles. Since the circumference of a circle is equal to the diameter or twice the radius, multiplied by 3.14159 (a number commonly denoted by the Greek letter $\pi$ ), we have the relation,

4 right angles $=360^{\circ}=2 \pi$ radians, or $180^{\circ}=\pi$ radians, therefore

and

$$
1^{\circ}=\pi / 180=0.0175 \text { radian }=17.5 \mathrm{mils},
$$

$$
1 \text { radian }=180^{\circ} / \pi=57.3^{\circ}, 1 \mathrm{mil}=60^{\prime} / 17.5=3.43^{\prime} \dagger .
$$

In reducing degrees to radians it is customary to retain the symbol $\pi$ in the answer unless there is some special reason for doing otherwise. Thus we write $60^{\circ}=\pi / 3$ radians rather than $60^{\circ}=1.047$ radians.

Again, when a number represents the measure of an angle and no unit is mentioned the natural unit is understood. Thus when we speak of the angle $\pi / 2$, we mean $\pi / 2$ radians not $\pi / 2$ degrees, the angle $\pi$ means $\pi$ radians or $180^{\circ}$ and not $\pi$ degrees. In fact we may look upon the symbols $\frac{1}{2} \pi, \pi, 2 \pi$, $2 n \pi$, etc., when referring to angles, as convenient abbreviations of $90^{\circ}, 180^{\circ}, 360^{\circ}, n$ times $360^{\circ}$, etc.

17.8. Relation between Angle, Arc, and Radius. In figure 39 let $\theta$ denote the radian measure of the angle, $r$ the

* See United States Army Engineers' Field Manual, Fifth Revised Edition, 1917, Government Printing Office, Washington, D. C.

$\dagger$ More accurately

$$
\begin{aligned}
& 1^{\circ}=0.017,453 \text { radian, } \quad 1 \text { radian }=57.295,770^{\circ} \text {. } \\
& 1^{\prime}=0.000,291 \text { radian. } \quad 1 \mathrm{mil}=3.437,747^{\prime} \text {. } \\
& \pi=3.141,592,654 \cdots
\end{aligned}
$$




\section{A SHORT COURSE IN MATHEMATICS}

radius, and $s$ the arc subtended by $\theta$. We have then by definition

$$
\theta=s / r, \text { and therefore } s=r \theta \text {, and } r=\bar{s} / \theta .
$$

From these equations, if any two of the three quantities $r$, $s, \theta$ are given, the third may be found. The equations are extremely useful in solving a great variety of

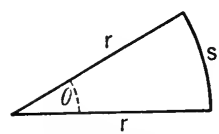

FIG. 39. interesting and important problems. It is important to keep in mind that $\theta$ must be given in radians (or must be reduced to radians) before these equations can be applied.

Example. The diameter of the sun subtends an angle of $32^{\prime}$ at the earth and the distance of the sun is about $93,000,000$ miles. Find the. approximate diameter of the sun.

Solution. The diameter of the sun will be approximately equal to the arc subtended by the angle $32^{\prime}$ at the distance of $93,000,000$ miles.

Now

$$
32^{\prime}=\frac{32^{\circ}}{60}=\frac{32}{60} \times \frac{\pi}{180} \text { radians, }
$$

hence

$$
\theta=\frac{32}{60} \times \frac{\pi}{180}, \text { and since } r=93,000,000 \text { miles, }
$$

diameter of $\operatorname{sun}=s=r \theta=93,000,000 \times \frac{32}{60} \cdot \frac{\pi}{180}$ miles $=866,000$ miles nearly.

17.9. Area of a Circular Sector. The area of a circular sector is equal to the area of a triangle with a base equal in length to the arc of the sector and an altitude equal to the radius, hence the area $A$ is

$$
A=r s / 2=r^{2} \theta / 2
$$

since by the preceding article $s=r \theta$. Again, in applying this formula, it must be remembered that $\theta$ is expressed in radians.

Example. Find the area of a circular sector whose radius is $100 \mathrm{ft}$. and the angle $15^{\circ}$. sq. ft.

Solution. $\quad 15^{\circ}=\pi / 12$ radians, hence $A=\frac{100 \times 100 \times \pi}{2 \times 12}$ sq. ft. $=1309$ 


\section{EXERCISE 17}

(To obtain the answers as given, use $\pi=3 \frac{1}{7}$.)

1. Express in radians each of the following angles :

$$
\begin{aligned}
& 90^{\circ}, 45^{\circ}, 22 \frac{1}{2}^{\circ}, 180^{\circ}, 225^{\circ}, 270^{\circ},-75^{\circ},-150^{\circ} . \\
& \text { Ans. } \pi / 2, \pi / 4, \pi / 8, \pi, 5 \pi / 4 \text {, etc. }
\end{aligned}
$$

2. State in which quadrant a line is which has described the following angles, the initial line coinciding with the positive direction of the $x$-axis : $105^{\circ},-105^{\circ}, 2 \pi / 3,-4 \pi / 3$, $2 n \pi+\pi / 4,2 n \pi-\pi / 4$.

3. Compute in radians one of the interior angles of an equilateral triangle; of a square; of a regular pentagon; hexagon; octagon. Ans. $\pi / 3, \pi / 2,3 \pi / 5$, etc.

4. What is the smallest positive angle coterminal with $465^{\circ}$ ? With $-\frac{4 \pi}{3} ?$

5. Find the complement of $\pi / 3-\theta$, of $-\theta$, of $\pi$. Find the supplements of these same angles.

6. The latitude of Seattle is $47^{\circ} 47^{\prime} \mathrm{N}$. Find the shortest distance from Seattle to the North Pole. (Distances on the earth are of course measured along ares of great circles. Use $r=3960$ miles.) Ans. 2926 miles.

7. How large an angle does one yard subtend at the distance of 1000 yards? One meter at a distance of 1000 meters? One mile at a distance of 1000 miles?

8. A fly-wheel $5 \mathrm{ft}$. in diameter makes 500 revolutions per minute. Find the velocity per second of a point on its rim.

$$
\text { Ans. } \frac{125 \pi}{3} \mathrm{ft} \text {. }
$$

9. Find the velocity per second of a point on the earth's equator owing to the rotation of the earth on its axis. (Use $r=3960$ miles.) 
10. Two railroad tracks (fig. 40) meet at $C$ at an angle of $120^{\circ}$. They are to be connected by a curve, the radius of the curve of the inner rail being $500 \mathrm{ft}$. (a) How long is the curve

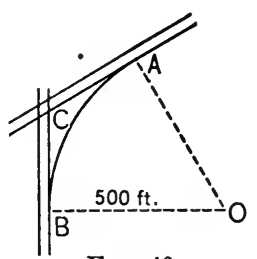

Fig. 40. of the inner rail? (b) How long is the curve of the outer rail, assuming the rails to be $5 \mathrm{ft}$. apart?

11. The diameter of a graduated circle is 12 inches, and the graduation is in mils. Find the distance between consecutive divisions on the rim.

12. A city lot has the shape of a circular sector with its curve bordering on the street. The straight sides of the lot are $150 \mathrm{ft}$. each and the angle between them is $60^{\circ}$. The price asked for the lot is $\$ 20$ per foot frontage. What is the price per acre?

Ans. \$11,616. 


\section{LESSON XVIII - THE TRIGONOMETRIC OR CIRCULAR FUNCTIONS}

18.1. Definition of Circular Functions. Let $O$ (fig. 41) represent the vertex, and $O A$ the initial side of any angle $\theta$. Take $O$ as the origin, and $O A$ as the positive direction of the $x$-axis of a rectangular coördinate system, and let $x, y$ represent the coördinates of any point $P$ on $O B$, the terminal side of the angle. Denote $O P$ by $r$. The ratio of any two of the three quantities, $x, y, r$, is independent of the position of the point $P$ on $O B$, but is dependent on the size of the angle $\theta$. These

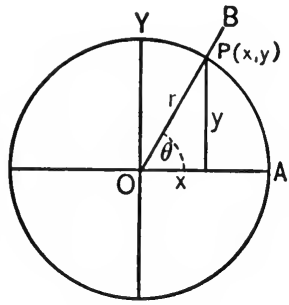

FIG. 41. ratios are therefore functions of the angle $\theta$. There are six such functions; they are defined and named as follows:

$\frac{y}{r}=\frac{\text { ordinate }}{\text { distance }}=\sin \theta$, which is read " the sine of the angle $\theta$ "; $\frac{x}{r}=\frac{\text { abscissa }}{\text { distance }}=\cos \theta$, which is read " the cosine of the angle $\theta$ "; $\frac{y}{x}=\frac{\text { ordinate }}{\text { abscissa }}=\tan \theta$, which is read " the tangent of the angle $\theta$ "; $\frac{r}{y}$, the reciprocal of the sine, is called the cosecant (csc) of the angle $\theta$;

$\frac{r}{x}$, the reciprocal of the cosine, is called the secant (sec) of the angle $\theta$;

$\frac{x}{y}$, the reciprocal of the tangent, is called the cotangent (cot) of the angle $\theta$. 
Together these functions are known as the trigonometric functions (trigon $=$ triangle + metron $=$ measure). They are also known by the name of circular functions, the reason for which will appear later.

\subsection{The Fundamental Relations between Circular Func-} tions. From the definitions of the circular functions it is plain that

$$
\begin{gathered}
\sin \theta \cdot \csc \theta=\frac{y}{r} \cdot \frac{r}{y}=1, \cos \theta \cdot \sec \theta=\frac{x}{r} \cdot \frac{r}{x}=1, \\
\tan \theta \cdot \cot \theta=\frac{y}{x} \cdot \frac{x}{y}=1 .
\end{gathered}
$$

Again, since $x^{2}+y^{2}=r^{2}$ (fig. 41), it follows that

$(\sin \theta)^{2}+(\cos \theta)^{2}=(y / r)^{2}+(x / r)^{2}=\left(y^{2}+x^{2}\right) / r^{2}=r^{2} / r^{2}=1$.

$(\tan \theta)^{2}+1=(y / x)^{2}+1=\left(y^{2}+x^{2}\right) / x^{2}=r^{2} / x^{2}=(r / x)^{2}=(\sec \theta)^{2}$. $(\cot \theta)^{2}+1=(x / y)^{2}+1=\left(x^{2}+y^{2}\right) / y^{2}=r^{2} / y^{2}=(r / y)^{2}=(\csc \theta)^{2}$.

Finally we have

$$
\frac{\sin \theta}{\cos \theta}=\frac{y / r}{x / r}=\frac{y}{x}=\tan \theta, \quad \frac{\cos \theta}{\sin \theta}=\frac{x / r}{y / r}=\frac{x}{y}=\cot \theta .
$$

Summing up we have

\section{Three Reciprocal Relations}

$$
\sin \theta \cdot \csc \theta=1, \quad \cos \theta \cdot \sec \theta=1, \quad \tan \theta \cdot \cot \theta=1 ;
$$

Three Square Relations

$\sin ^{2} \theta+\cos ^{2} \theta=1, \quad \tan ^{2} \theta+1=\sec ^{2} \theta, \quad \cot ^{2} \theta+1=\csc ^{2} \theta ;$ Two Quotient Relations

$$
\tan \theta=\sin \theta / \cos \theta, \quad \cot \theta=\cos \theta / \sin \theta ;
$$

where, as is customary, we have written $\sin ^{2} \theta$ for $(\sin \theta)^{2}$, $\cos ^{2} \theta$ for $(\cos \theta)^{2}$, etc.

18.3. Trigonometric Identities. Like algebraic expressions, expressions involving trigonometric functions may frequently be reduced to simpler forms. As a rule the reduction 
is most easily effected by expressing each of the functions which occur in terms of the sine and cosine and by reducing the resultant expression like any algebraic expression, treating the sine and cosine as two separate quantities.

Two or more trigonometric expressions which may be reduced to the same form are called trigonometric identities.

Example 1. Reduce to its simplest form the expression $\sin ^{2} \theta \tan \theta+\cos ^{2} \theta \cot \theta+2 \sin \theta \cos \theta$.

Solution. Since $\tan \theta=\sin \theta / \cos \theta$, and $\cot \theta=\cos \theta / \sin \theta$, we may write

$\sin ^{2} \theta \frac{\sin \theta}{\cos \theta}+\cos ^{2} \theta \frac{\cos \theta}{\sin \theta}+2 \sin \theta \cos \theta=\frac{\sin ^{4} \theta+\cos ^{4} \theta+2 \sin ^{2} \theta \cos ^{2} \theta}{\sin \theta \cos \theta}$ $=\frac{\left(\sin ^{2} \theta+\cos ^{2} \theta\right)^{2}}{\sin \theta \cos \theta}=\frac{1}{\sin \theta \cos \theta}=\csc \theta \sec \theta$, since $\sin ^{2} \theta+\cos ^{2} \theta=1$.

Example 2. Show that

$\sin A \tan ^{2} A+\csc A \sec ^{2} A=2 \tan A \sec A+\csc A-\sin A$.

Solution. Put for $\tan A, \csc A$, and $\sec A$ their values in terms of $\sin A$ and $\cos A$. Then the left member of the identity becomes

$\frac{\sin ^{3} A}{\cos ^{2} A}+\frac{1}{\sin A \cos ^{2} A}=\frac{\sin ^{4} A+1}{\sin A \cos ^{2} A}$, and the right member becomes

$2 \frac{\sin A}{\cos ^{2} A}+\frac{1}{\sin A}-\sin A=\frac{2 \sin ^{2} A+\cos ^{2} A-\sin ^{2} A \cos ^{2} A}{\cos ^{2} A \sin A}$

$=\frac{\sin ^{2} A-\sin ^{2} A \cos ^{2} A+\left(\sin ^{2} A+\cos ^{2} A\right)}{\cos ^{2} A \sin A}=\frac{\sin ^{2} A\left(1-\cos ^{2} A\right)+1}{\cos ^{2} A \sin A}$

$=\frac{\sin ^{4} A+1}{\cos ^{2} A \sin A}$, since $1-\cos ^{2} A=\sin ^{2} A$.

\section{EXERCISE 18}

Reduce the following trigonometric expressions to their simplest form.

1. $\sec \theta-\tan \theta \sin \theta$.

Ans. $\cos \theta$.

2. $\sin ^{4} x+\cos ^{4} x+2 \sin ^{2} x \cos ^{2} x$.

3. $\cot A-\sec A \csc A\left(1-2 \sin ^{2} A\right)$.

Ans. $\tan A$.

4. $\frac{\tan A+\tan B}{\cot A+\cot B}$. Ans. $\tan A \tan B$. 
5. $(x \cos \alpha-y \sin \alpha)^{2}+(x \sin \alpha+y \cos \alpha)^{2}$. Ans. $x^{2}+y^{2}$. Prove the following identities:

6. $\sin ^{4} x-\cos ^{4} x=\sin ^{2} x-\cos ^{2} x=1-2 \cos ^{2} x=2 \sin ^{2} x-1$.

7. $\frac{\cot A \cos A}{\cot A+\cos A}=\frac{\cot A-\cos A}{\cot A \cos A}$.

8. $(\sin B+\cos B)(\tan B+\cot B)=\sec B+\csc B$.

9. $\cos ^{4} x-\sin ^{4} x=\cos ^{2} x(1-\tan x)(1+\tan x)$.

10. $(\cos \alpha \cos \beta+\sin \alpha \sin \beta \cos C)^{2}$

$+(\sin \alpha \cos \beta-\cos \alpha \sin \beta \cos C)^{2}=1-\sin ^{2} \beta \sin ^{2} C$. 
LESSON XIX-REDUCTIONS TO THE FIRST QUADRANT

19.1. The Signs of the Functions. An angle, like angle $A O P_{1}$ (fig. 42), whose terminal side falls in the first quadrant (the vertex being at the origin and the initial line lying along the positive $x$-axis), is called an angle in the first quadrant. An angle, like $A O P_{2}$, whose terminal side falls in the second quadrant, is called an angle in the second quadrant. Similarly, the angles $\mathrm{AOP}_{3}$ and $A O P_{4}$ are angles in the third and fourth quadrants respectively.

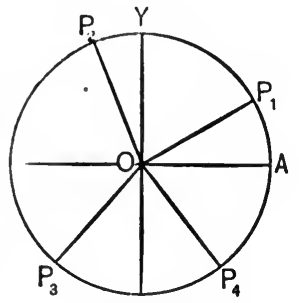

Fra. 42.

(a) Since the sine of an angle is the ratio of the ordinate of $P$ to its distance from $O$, and this distance is always positive, the sign of the sine is the same as the sign of the ordinate, which is + in the first and second, - in the third and fourth quadrants.

(b) Since the cosine is the ratio of the abscissa of $P$ to its distance from $O$, and the distance is always positive, the sign of the cosine is the same as the sign of the abscissa, which is +. in the first and fourth, - in the second and third quadrants.

(c) Since the tangent is the ratio of the ordinate of $P$ to the abscissa of $P$, the sign of the tangent is + when the ordinate and abscissa have like signs, that is, in the first and third quadrants, and - when they have unlike signs, that is, in the second and fourth quadrants. 
(d) Any number and its reciprocal have like signs, hence the signs of the cosecant, secant and cotangent are the same as
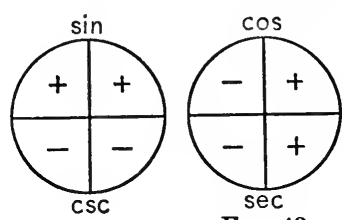

Frg. 43.

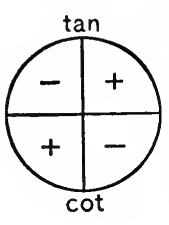

the signs of the sine, cosine, and tangent respectively.

The student should make himself perfectly familiar with the signs of the functions in the various quadrants. Figure 43 will prove an aid to the memory.

19.2. Periodicity of the Circular Functions. If the terminal side of an angle is revolved in the plane of the angle through four right angles, or any number of times four right angles, it will return to the position from which it started, and this is true whether the revolution is in the positive (counter-clockwise) or negative (clockwise) direction. It follows that the trigonometric functions of any angle remain unchanged when the angle is increased or decreased by four right angles, or by any number of times four right angles. Therefore, if we use $R$ to denote a right angle, and $n$ any integer, we have

$$
\begin{aligned}
& \sin (\theta \pm 4 n R)=\sin \theta \\
& \cos (\theta \pm 4 n R)=\cos \theta \\
& \tan (\theta \pm 4 n R)=\tan \theta
\end{aligned}
$$

and similar equations for the cosecant, secant, and cotangent.

Examples.

$\sin 365^{\circ}=\sin 5^{\circ}, \quad \tan 737^{\circ} 15^{\prime}=\tan 17^{\circ} 15^{\prime}, \quad \cos -265^{\circ}=\cos 95^{\circ}$,

$$
\sin \left(6 \pi+\frac{\pi}{3}\right)=\sin \frac{\pi}{3}, \csc (7 \pi)=\csc \pi, \cot \left(\frac{\pi}{4}-8 \pi\right)=\cot \frac{\pi}{4} .
$$

It should be observed that by means of these formulas any function of any angle, whether positive or negative, can be replaced by the same function of some positive angle less than four right angles. 
Since the trigonometric functions remain unchanged when the angle is increased or diminished by $4 R$, they are called periodic functions with a period $4 R$ or $2 \pi$. It will be seen presently that the tangent and cotangent have the smaller period $2 R$ or $\pi$.

19.3. Angles in the Second Quadrant. We will now show that any function of any angle can be expressed in terms of the same function of some angle in the first quadrant, that is, in terms of some angle not greater than $90^{\circ}$.

We will first consider angles in the second quadrant. Let $A O P_{2}$ (fig. 44) be any angle in the second quadrant; $O P_{2}$ a fixed distance, and let $O P_{1}$ be drawn symmetrical to $O P_{2}$ with respect to the $y$-axis; then if we denote angle $A O P_{1}$ by $\theta$, angle $A O P_{2}$ equals $2 R-\theta$.

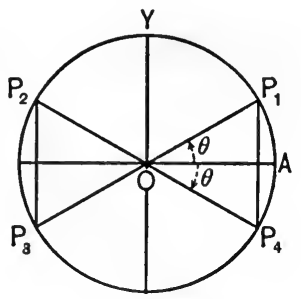

FIG. 44.

Since $P_{1}$ and $P_{2}$ are symmetrical with respect to the $y$-axis, the ordinates of $P_{1}$ and $P_{2}$ are equal while their abscissas are equal but have opposite signs; therefore

$$
\begin{aligned}
& \sin (2 R-\theta)=+\sin \theta, \\
& \cos (2 R-\theta)=-\cos \theta, \\
& \tan (2 R-\theta)=-\tan \theta .
\end{aligned}
$$

Now $2 R-\theta$ is the angle $A O P_{2}$. We see therefore that the functions of any angle in the second quadrant can be expressed in terms of the angle $\theta$ which is less than $R$ or $90^{\circ}$. Moreover, since $2 R-\theta$ and $\theta$ are supplementary angles, we have

The sines of supplementary angles are equal, while the cosines and tangents of supplementary angles are equal with opposite signs. 
Examples. $\quad \sin 3 R / 2=\sin (2 R-R / 2)=\sin R / 2$,

$$
\begin{aligned}
\cos 170^{\circ} & =\cos \left(180^{\circ}-10^{\circ}\right)=-\cos 10^{\circ}, \\
\tan 5 \pi / 6 & =\tan (\pi-\pi / 6)=-\tan \pi / 6 .
\end{aligned}
$$

19.4. Angles in the Third Quadrant. Let $A O P_{3}$ (fig. 44) be any angle in the third quadrant, $O P_{3}$ any fixed distance, and let $O P_{1}$ be drawn symmetrical to $O P_{3}$ with respect to the origin; then if we denote angle $A O P_{1}$ by $\theta$, angle $A O P_{3}=$ $2 R+\theta$.

Since the points $P_{1}$ and $P_{3}$ are symmetrically situated with respect to the origin, their ordinates are equal with opposite signs, and so are their abscissas; therefore

$$
\begin{gathered}
\sin (2 R+\theta)=-\sin \theta, \\
\cos (2 R+\theta)=-\cos \theta, \\
\tan (2 R+\theta)=+\tan \theta .
\end{gathered}
$$

From the last one of these equations it is seen that the tangent of an angle remains unchanged when the angle is increased by $2 R=180^{\circ}$. The same is true of its reciprocal, the cotangent. These two functions have therefore the period $2 R$ or $\pi$, as was stated in $\mathbf{1 9 . 2}$.

Examples. $\quad \sin 225^{\circ}=\sin \left(180^{\circ}+45^{\circ}\right)=-\sin 45^{\circ}$,

$$
\begin{aligned}
\cos 7 \pi / 6 & =\sin (\pi+\pi / 6)=-\sin \pi / 6, \\
\tan (5 R / 2) & =\tan \left(2 R+\frac{1}{2} R\right)=\tan \frac{1}{2} R .
\end{aligned}
$$

19.5. Angles in the Fourth Quadrant. Let $A O P_{4}$ (fig. 44) be any angle in the fourth quadrant, $\mathrm{OP}_{4}$ a fixed distance, and let $O P_{1}$ be drawn symmetrical to $O P_{4}$ with respect to the $x$-axis ; then if we denote $A O P_{1}$ by $\theta$, angle $A O P_{4}=4 R-\theta$. $P_{1}$ and $P_{4}$, being symmetrically situated with respect to the $x$-axis, have equal abscissas and ordinates which differ only in sign; therefore

$$
\begin{array}{r}
\sin (4 R-\theta)=-\sin \theta \\
\cos (4 R-\theta)=+\cos \theta \\
\tan (4 R-\theta)=-\tan \theta
\end{array}
$$


Examples. $\quad \sin 300^{\circ}=\sin \left(360^{\circ}-60^{\circ}\right)=-\sin 60^{\circ}$, $\cos 300^{\circ}=\cos \left(360^{\circ}-60^{\circ}\right)=\cos 60^{\circ}$, $\tan 300^{\circ}=\tan \left(360^{\circ}-60^{\circ}\right)=-\tan 60^{\circ}$.

19.6. Negative Angles. The angle $-\theta$ (angle $A O P_{4}$ in figure 44 ) is coterminal with $4 R-\theta$; hence we have from the formulas in $\mathbf{1 9 . 5}$

$\sin (-\theta)=-\sin \theta, \quad \cos (-\theta)=+\cos \theta, \quad \tan (-\theta)=-\tan \theta$.

19.7. Complementary Angles. Let $A O P$ (fig. 45) be any angle in the first quadrant; then $P O B$ is its complement. If we denote angle $P O B$ by $\theta$, then $A O P=90^{\circ}-\theta=R-\theta$. Construct angle $A O Q=P O B=\theta$ and take $O Q=O P$. Draw $P C$ and $Q D$ perpendicular to $O A$. Then triangle $O P C=$ triangle $O Q D$ and

$$
\begin{aligned}
& \sin (\boldsymbol{R}-\boldsymbol{\theta})=C P / O P=O D / O Q=\cos \theta \\
& \tan (\boldsymbol{R}-\boldsymbol{\theta})=C P / O C=O D / D Q=\cot \theta \\
& \sec (\boldsymbol{R}-\boldsymbol{\theta})=O P / O C=O Q / D Q=\csc \theta \\
& \cos (\boldsymbol{R}-\boldsymbol{\theta})=O C / O P=D Q / O Q=\sin \theta \\
& \cot (\boldsymbol{R}-\boldsymbol{\theta})=O C / C P=D Q / O D=\tan \theta \\
& \csc (\boldsymbol{R}-\boldsymbol{\theta})=O P / C P=O Q / O D=\sec \theta
\end{aligned}
$$

that is to say,

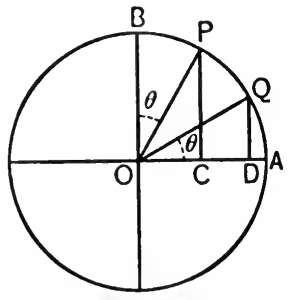

Fig. 45.

The sine of an angle is equal to the cosine of its complement and conversely, the tangent of an angle is equal to the cotangent of its complement and conversely, the secant of an angle is equal to the cosecant of its complement and conversely.

For this reason the sine and cosine are called complementary functions; each is said to be the cofunction of the other. Likewise, and for the same reason, the tangent and cotangent, and the secant and cosecant, are pairs of complementary functions.

We have taken the angle $A O P$ in the first quadrant, but it 
may be shown that the formulas of this article hold true for angles in any quadrant.

Examples. $\quad \sin 65^{\circ}=\sin \left(R-25^{\circ}\right)=\cos 25^{\circ}$,

$$
\begin{aligned}
& \cos 60^{\circ}=\cos \left(90^{\circ}-30^{\circ}\right)=\sin 30^{\circ}, \\
& \tan 85^{\circ}=\tan \left(90^{\circ}-5^{\circ}\right)=\cot 5^{\circ},
\end{aligned}
$$

. $\cot 105^{\circ}=\cot \left[90^{\circ}-\left(-15^{\circ}\right)\right]=\tan \left(-15^{\circ}\right)=-\tan 15^{\circ}$, etc.

19.8. Use of the Reduction Formulas. Formulas in $\mathbf{1 9 . 2}$ to 19.6 inclusive enable us to express the functions of any angle in terms of the functions of angles in the first quadrant, that is, in terms of acute angles: Consequently when the functions of acute angles have been computed we can find the functions of any other angle in terms of these without any new computations. In fact by virtue of 19.7 the functions of any angle can be expressed in terms of functions of angles not greater than $45^{\circ}$, for if an acute angle is greater than $45^{\circ}$ its complement is less than $45^{\circ}$.

\section{EXERCISE 19}

1. Express the sine, cosine, and tangent of each of the following angles in terms of acute angles: (a) $125^{\circ}$, (b) $235^{\circ}$, (c) $316^{\circ},(d) 415^{\circ},(e) 739^{\circ},(f)-14^{\circ},(g)-94^{\circ},(h)-197^{\circ}$, (i) $-345^{\circ}$.

2. Express the sine, cosine, and tangent of each of the following angles in terms of angles not greater than $45^{\circ}$ : (a) $67^{\circ}$,
(b) $107^{\circ}$,
(c) $215^{\circ}$,
(d) $265^{\circ}$,
(e) $290^{\circ}$,
$(f)-56^{\circ}$,
(g) $-105^{\circ}$
(h) $-675^{\circ}$, (i) $4576^{\circ}$.

3. Prove each of the following formulas:

(a) $\sin (R+\theta)=\cos \theta, \quad \cos (R+\theta)=-\sin \theta$,

$\tan (R+\theta)=-\cot \theta$;

(b) $\sin (3 R-\theta)=-\cos \theta, \quad \cos (3 R-\theta)=-\sin \theta$, $\tan (3 R-\theta)=\cot \theta$; 
(c) $\sin (3 R+\theta)=-\cos \theta, \quad \cos (3 R+\theta)=\sin \theta$, $\tan (3 R+\theta)=-\cot \theta$;

(d) $\sin (\theta-R)=-\cos \theta, \quad \cos (\theta-R)=\sin \theta$, $\tan (\theta-R)=-\cot \theta$;

(e) $\sin (\theta-2 R)=-\sin \theta, \quad \cos (\theta-2 R)=-\cos \theta$, $\tan (\theta-2 R)=-\tan \theta$

(f) $\sin (\theta-3 R)=\cos \theta, \quad \cos (\theta-3 R)=-\sin \theta$, $\tan (\theta-3 R)=-\cot \theta$.

4. Prove that all the formulas in 19.2, 19.3, 19.4, 19.5, and 19.6 hold true if we write cosecant for sine, secant for cosine, and cotangent for tangent.

5. Commit to memory the formulas in $\mathbf{1 9 . 3}$ and 19.7. 


\section{LESSON XX-FUNCTIONS OF ANGLES IN THE FIRST QUADRAN'}

20.1. Restatement of Definitions. We have seen in the preceding sections how the functions of any angle, positive or negative, may be expressed in terms of the functions of an angle in the first quadrant. We must now study the func-

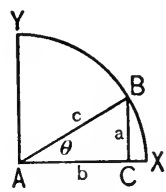

Fig. 46. tions of an angle in the first quadrant more in detail.

Let $A B C$ be any right triangle, $A$ and $B$ the acute angles, $a$ and $b$ the sides opposite these angles, $C$ the right angle and $c$ the hypotenuse. If the vertex $A$ is chosen as the origin and $A C$ as the axis of abscissas, the coördinates of the point $B$ are $b$ and $a$ and its distance from $A$ is $c$. The angle $A(=\theta)$ is an angle in the first quadrant, and we have by 18.1,

$\sin A=\frac{\text { ordinate }}{\text { distance }}=\frac{a}{c}, \cos A=\frac{\text { abscissa }}{\text { distance }}=\frac{b}{c}, \tan A=\frac{\text { ordinate }}{\text { abscissa }}=\frac{a}{b} ;$ in words: In any right triangle

The sine of an acute angle is equal to the side opposite the angle divided by the hypotenuse.

The cosine of an acute angle is equal to the side adjacent to the angle divided by the hypotenuse.

The tangent of an acute angle is equal to the side opposite the angle divided by the side adjacent to the angle.

These definitions are more convenient than the definitions in 18.1, but they apply only when the angle is in the first 
quadrant, for a right triangle can not have an angle greater than $90^{\circ}$.

20.2. Natural Functions Tables.* The values of the principal functions (sines, cosines, tangents) for every degree, minute, and second from $0^{\circ}$ to $90^{\circ}$ have been computed once for all and the results tabulated in tables, known as tables of natural functions. Usually such tables contain the sines, cosines, tangents and cotangents of angles differing by one minute; in some tables the angles differ by 10 seconds, and in still others by only one second. From such tables the value of the sine, cosine, tangent or cotangent of any angle may be found whenever needed, and conversely, when the value of the function is known the corresponding value of the angle may be found from the table. Secants and cosecants are not usually given directly by the tables, but may be found indirectly from the sines and cosines, from the relations $\sec \theta=1 / \cos \theta, \csc \theta=1 / \sin \theta$.

There are however a few angles $\left(0^{\circ}, 30^{\circ}, 45^{\circ}, 60^{\circ}, 90^{\circ}\right)$ which occur so frequently that it is worth while for the student to remember their functions once for all. This is not at all difficult if it is kept in mind how these functions are derived, as we shall now show.

20.3. Functions of $45^{\circ}$. When the angle $A$ (fig. 47 ) is $45^{\circ}$, the right triangle $A B C$ is isosceles, that is, the two sides $a$ and $b$ are equal, and then $c^{2}=a^{2}+b^{2}=a^{2}+a^{2}=2 a^{2}$, from which $c=a \sqrt{ } 2$.

We then have

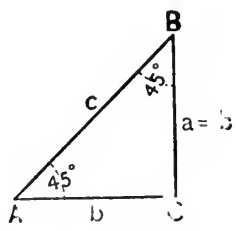

Fig. 47. $\sin 45^{\circ}=\frac{a}{a \sqrt{ } 2}=\frac{1}{2} \sqrt{ } 2, \cos 45^{\circ}=\frac{a}{a \sqrt{ } 2}=\frac{1}{2} \sqrt{ } 2, \tan 45^{\circ}=\frac{a}{a}=1$.

* For the purposes of the problems in this text the student is advised to provide himself with Macmillan's Logarithmic and Trigonometric Tables, Macmillan Co., New York (1918). 


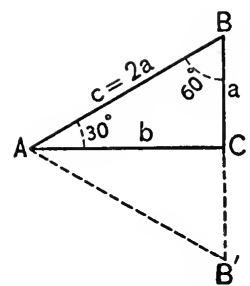

FIG. 48.

20.4. Functions of $30^{\circ}$ and $60^{\circ}$. When the angle $A$ (fig. 48 ) is $30^{\circ}$, the other angle of the right triangle $A B C$ equals $60^{\circ}$; this triangle then forms one-half of an equilateral triangle and therefore $c=2 a$ and $b^{2}=c^{2}-a^{2}=(2 a)^{2}$ $-a^{2}=3 a^{2}$, from which $b=a \sqrt{ } 3$. We then have

$\sin 30^{\circ}=\frac{a}{c}=\frac{a}{2 a}=\frac{1}{2}, \quad \cos 30^{\circ}=\frac{a \sqrt{ } 3}{c}=\frac{a \sqrt{ } 3}{2 a}=\frac{1}{2} \sqrt{ } 3$,

$\tan 30^{\circ}=\frac{a}{a \sqrt{ } 3}=\frac{1}{\sqrt{ } 3}=\frac{1}{3} \sqrt{ } 3, \quad \cot 30^{\circ}=\frac{a \sqrt{ } 3}{a}=\sqrt{ } 3$.

Also by 19.7 or directly from figure 48 we have

$$
\begin{gathered}
\sin 60^{\circ}=\cos 30^{\circ}=\frac{1}{2} \sqrt{ } 3, \quad \cos 60^{\circ}=\sin 30^{\circ}=\frac{1}{2}, \\
\tan 60^{\circ}=\cot 30^{\circ}=\sqrt{ } 3 .
\end{gathered}
$$

20.5. Functions of $0^{\circ}$ and $90^{\circ}$. In figure 49 let $\theta$ be a variable angle formed by rotating the $O P$ of unit length about $O$ as a center. Then

$$
\begin{aligned}
& \sin \theta=C P / O P=C P / 1=C P, \\
& \cos \theta=O C / O P=O C / 1=O C, \\
& \tan \theta=A T / O A=A T / 1=A T .
\end{aligned}
$$

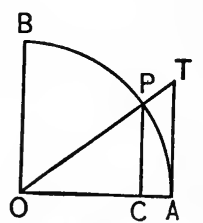

Fra. 49 .

As the angle $\theta$ decreases, $C P$ decreases, $O C$ increases, and $A T$ decreases; as $\theta$ approaches zero, $C P$ approaches zero, $O C$ approaches $O A$, that is unity, and $A T$ approaches zero. These relations are briefly and conveniently expressed by sayịng,

$$
\sin 0^{\circ}=0, \quad \cos 0^{\circ}=1, \quad \tan 0^{\circ}=0 .
$$

Again, as the angle $\theta$ increases, $C P$ increases, $O C$ decreases, and $A T$ increases; as $\theta$ approaches $90^{\circ}, C P$ approaches $O B$ or unity, $O C$ approaches zero, and $A T$ becomes larger than any number that may be mentioned. These relations are briefly expressed as follows

$$
\sin 90^{\circ}=1, \quad \cos 90^{\circ}=0, \quad \tan 90^{\circ}=\infty .
$$


It should be remembered that the symbol $\infty$ (infinite) represents no finite number whatever. What we mean by saying that the tangent of $90^{\circ}$ is infinite is this and nothing more: As a variable angle approaches sufficiently near to $90^{\circ}$ its tangent becomes larger than any fixed number however large.

The results of this and the preceding sections are summarized in the following table which the student should memorize once for all.

\begin{tabular}{|cccccc|}
\hline & $0^{\circ}$ & $30^{\circ}$ & $45^{\circ}$ & $60^{\circ}$ & $90^{\circ}$ \\
$\sin$ & 0 & $\frac{1}{2}$ & $\frac{1}{2} \sqrt{2}$ & $\frac{1}{2} \sqrt{ } 3$ & 1 \\
$\cos$ & 1 & $\frac{1}{2} \sqrt{ } 3$ & $\frac{1}{2} \sqrt{ } 2$ & $\frac{1}{2}$ & 0 \\
$\tan$ & 0 & $\frac{1}{3} \sqrt{ } 3$ & 1 & $\sqrt{ } 3$ & $\infty$ \\
\hline
\end{tabular}

20.6. Trigonometric Lines. In figure 50 let $A O P_{1}$ be any angle in the first quadrant. If $O A$ is kept fixed while $O P$ is revolved about $O$, every point in $O P$ will describe a circle about $O$ as a center. Consider the circle whose radius is $O P_{1}=1$. At the point where the initial line intersects the circle draw a tangent to the circle and prolong $O P_{1}$ to meet this tangent line as at $T_{1}$. Draw $P_{1} C_{1}$ perpendicular to $O A$. Then if we denote the angle $A O P_{1}$ by $\theta$, we have

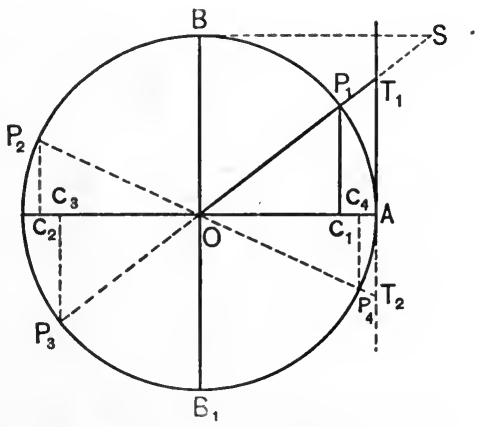

FIG. 50. - Trigonometric Lines.

$$
\begin{aligned}
& \sin \theta=C_{1} P_{1} / O P_{1}=C_{1} P_{1} / 1=C_{1} P_{1}, \\
& \cos \theta=O C_{1} / O P_{1}=O C_{1} / 1=O C_{1}, \\
& \tan \theta=C_{1} P_{1} / O C_{1}=A T_{1} / O A=A T_{1} / 1=A T_{1} .
\end{aligned}
$$

It is plain, therefore, that if the circle is drawn on closely ruled coördinate paper, the approximate values of the sine, 
cosine, and tangent may be read off directly from the figure, namely,

$$
C_{1} P_{1}=\sin \theta, \quad O C_{1}=\cos \theta, \quad A T_{1}=\tan \theta .
$$

For angles in the second quadrant, $A O P_{2}=\theta_{2}$ we have

$$
C_{2} P_{2}=\sin \theta_{2}, \quad O C_{2}=\cos \theta_{2}, \quad A T_{2}=\tan \theta_{2} .
$$

For angles in the third quadrant, $A O P_{3}=\theta_{3}$,

$$
C_{3} P_{3}=\sin \theta_{3}, \quad O C_{3}=\cos \theta_{3}, \quad A T_{1}=\tan \theta_{3} .
$$

For angles in the fourth quadrant, $A O P_{4}=\theta_{4}$,

$$
C_{4} P_{4}=\sin \theta_{4}, \quad O C_{4}=\cos \theta_{4}, \quad A T_{2}=\tan \theta_{4} .
$$

The lines $C P, O C$, and $A T$ taken with reference to a unit circle might be used to define the trigonometric functions. In fact they were formerly so defined and were called circular functions because of the relations of these lines to the circle, a term which is still frequently used.

20.7. Variation of the Functions. The trigonometric lines are very convenient in following the changes in the functions as the angle changes. Let the angle $\theta$ increase from 0 until it reaches $360^{\circ}$. What are the corresponding changes in $C P, O C$, and $A T$ ? Plainly $C P$ increases beginning with 0 until it reaches 1 at $B$, then it decreases again reaching 0 , when the angle reaches $180^{\circ}$, and continues to decrease until it reaches the extreme value -1 at $B_{1}$, after which it increases, reaching its original value 0 when the angle is $360^{\circ}$. In like manner the changes in $O C$, the cosine, and $A T$, the tangent, may be easily traced as the angle changes from 0 to $360^{\circ}$.

\section{EXERCISE 20}

1. Given $\sin A=\frac{3}{5}$, find all the other functions of $A$. Ans. $\cos \dot{A}=\frac{4}{5}, \tan A=\frac{3}{4}, \sec A=\frac{5}{4}, \csc A=\frac{5}{3}, \cot A=\frac{4}{8}$.

2. Given $\tan A=3$, find all the other functions of $A$.

3. Given $\sec A=5$, find the other functions of $A$. 
4. Make out a table like the one given in $\mathbf{2 0 . 5}$, giving the secant, cosecant, and cotangent of each of the angles $0^{\circ}, 30^{\circ}$, $45^{\circ}, 60^{\circ}, 90^{\circ}$.

5. Use figure 50 to follow the changes in the tangent of $\theta$ as $\theta$ changes from $0^{\circ}$ to $360^{\circ}$.

6. Show that $O T_{1}$ (fig. 50) represents the $\sec \theta$.

7. If $S$ represents the point (fig. 50) in which the tangent to the circle at $B$ meets $O P$ prolonged, show that $B S=\cot \theta$ and $O S=\csc \theta$, the radius of the circle being unity.

8. $\operatorname{Sin} 45^{\circ}=\frac{1}{2} \sqrt{ } 2$, find the other angle less than $360^{\circ}$ for which the sine is $\frac{1}{2} \sqrt{ } 2$.

9. What other angle less than $360^{\circ}$ has the same cosine as $30^{\circ}$ ?

10. What other angle less than $360^{\circ}$ has the same tangent as $60^{\circ}$ ?

11. By the method suggested in 20.6 find the approximate values of the sines, cosines, and tangents of $15^{\circ}$ and of $22 \frac{1}{2}^{\circ}$. Compare your results with those given in the tables. 


\section{LESSON XXI-TRIGONOMETRIC GRAPHS}

21.1. The Sine Curve in the First Quadrant. Like every other function of $x$ the function $y=\sin x$ may be represented by a graph. To construct this graph we first find the values of $y$ corresponding to a succession of values of $x$. The natural functions table gives the value of $y$ for any value of $x$ in the first quadrant, that is, of any angle less than $90^{\circ}$. We thus find the following corresponding values of $x$ and $y$ :

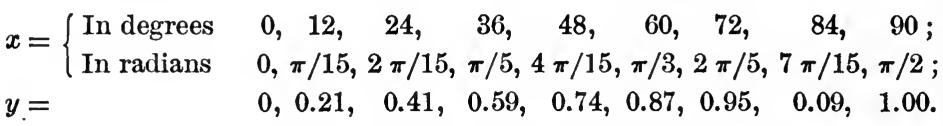

Each pair of values $x$ and $y$ determines a point on the sine curve. To plot these points we may take for abscissas the

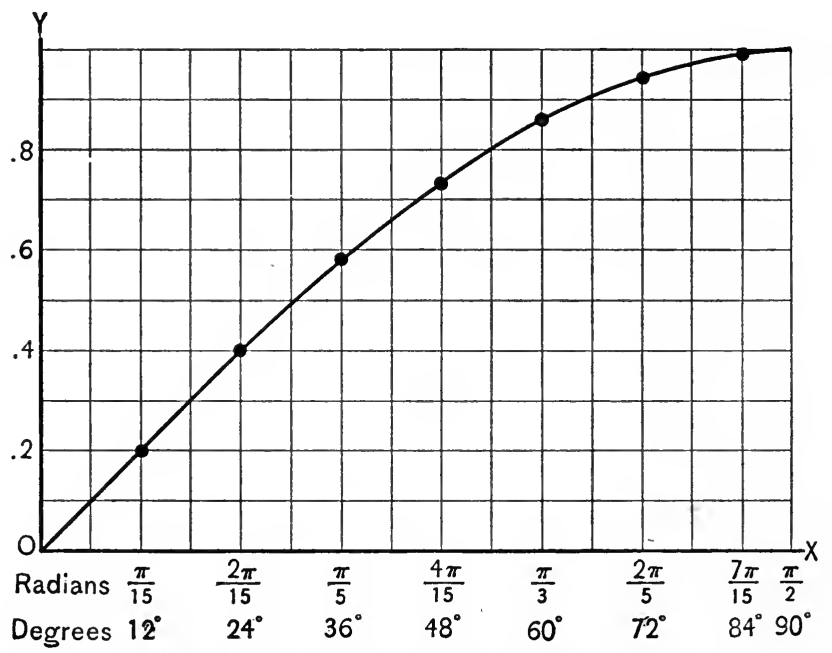

Fia. 51. - Sine Curve in the First Quadrant. 
angles expressed in either degrees or radians. The latter involve the number $\pi=3.1416 \ldots$. If therefore we choose $\pi / 3$ as the unit along the $x$-axis, the scale of abscissas will differ but slightly from the scale of ordinates, while we shall avoid the awkward fractions which would arise if we used exactly the same unit for both scales. Plotting the points as indicated and connecting them by a smooth curve we obtain the sine curve for the first quadrant (fig. 51).

21.2. The Complete Sine Curve. It is now easy to complete the sine curve as shown in figure 52 .

Second Quadrant. Since $\sin (\pi-\theta)=\sin \theta$, ordinates equally distant from $Q_{1}$ are equal, that is, the curve is symmetrical with respect to the ordinate at $Q_{1}$. Therefore, as $x$ varies from $\pi / 2$ to $\pi$, the curve descends, crossing the $x$-axis again at $\pi$.

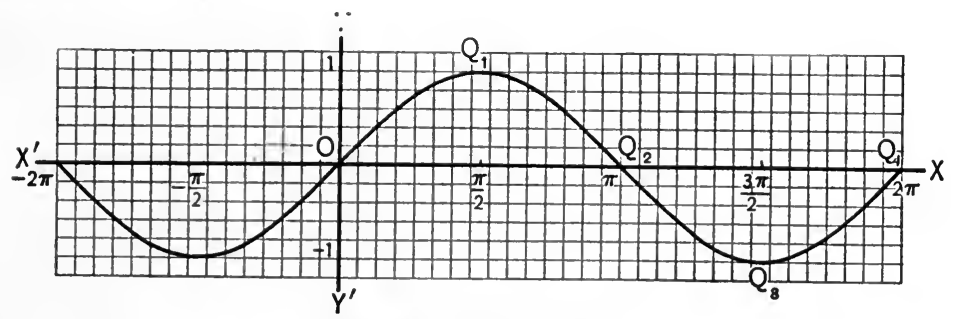

Fra. 52. - Sine Curve.

Third and Fourth Quadrants. Since $\sin (2 \pi-\theta)=-\sin \theta$, ordinates equally distant from $Q_{4}$ and $O$ are numerically equal but have opposite signs. The curve from $x=\pi$ to $x=2 \pi$ is therefore exactly like the portion of the curve in the first and second quadrants when revolved about the $x$-axis through an angle of $180^{\circ}$.

21.3. Periodicity, Wave-length, and Amplitude. Since $\sin (\theta \pm 2 \pi)=\sin \theta$, the sine curve will repeat itself as $x$ varies 
from $2 \pi$ to $4 \pi$, and again as $x$ varies from $4 \pi$ to $6 \pi$, and so on indefinitely. Similarly the curve will repeat itself as $x$ varies from 0 to $-2 \pi$, and again as $x$ varies from $-2 \pi$ to $-4 \pi$, and so on. That is to say, since the function $y=\sin x$ is a periodic function with the period $2 \pi$, the sine curve is a periodic curve with the period $2 \pi$.

The distance between two consecutive points at which the sine curve crosses the $x$-axis in the same direction, as $O Q_{4}$ in figure 52, is called the wave-length of the curve. The greatest height of the curve, represented by the ordinate at $Q_{1}$ in figure 52, is called the amplitude of the curve.

The conclusions of this article may be summed up by saying :

The sine curve, $y=\sin x$, is a periodic curve whose wavelength is $2 \pi$ and whose amplitude is 1 .

21.4. Sine Curves with Different Amplitudes. If we lengthen or shorten each ordinate of the sine curve in the

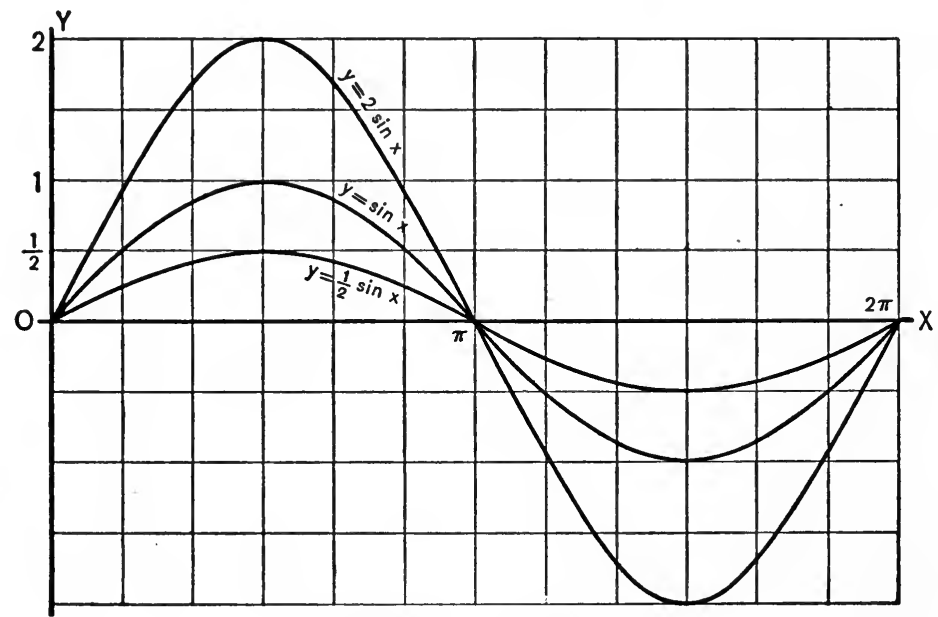

Fia. 53. $-y=a \sin x$. 
ratio $a$ to 1 we obtain a new curve whose equation is $y=$ $a \sin x$. This new curve is a sinusoid (sine-like curve) whose wave-length is $2 \pi$ and whose amplitude is $a$. Figure 53 shows three curves, the sine curve $y=\sin x$, and two sinusoids whose equations are $y=2 \sin x$, and $y=\frac{1}{2} \sin x$, respectively.

21.5. Sine Curves with Different Wave-lengths. Let us next consider the curve $y=a \sin k x$. This curve has the same amplitude as the curve $y=a \sin x$, but its wave-length is different from $2 \pi$, for $y$ equals 0 when

that is, when

$$
\begin{aligned}
& k x=0, \text { or } \pi, \quad \text { or } 2 \pi, \quad \text { or } 3 \pi \quad \text { etc., } \\
& x=0 \text {, or } \pi / k \text {, or } 2 \pi / k \text {, or } 3 \pi / k \text {, etc. }
\end{aligned}
$$

The distance between consecutive intersection points of the curve with the $x$-axis in the same direction is therefore $2 \pi / k$, that is, the wave length of the curve $y=a \sin k x$ is $2 \pi / k$. If we denote this wave-length by $\lambda=2 \pi / k$, we have $k=2 \pi / \lambda$, and the equation of the curve may be written

$$
y=a \sin 2 \pi x / \lambda,
$$

where $a$ is the amplitude and $\lambda$ the wave-length.

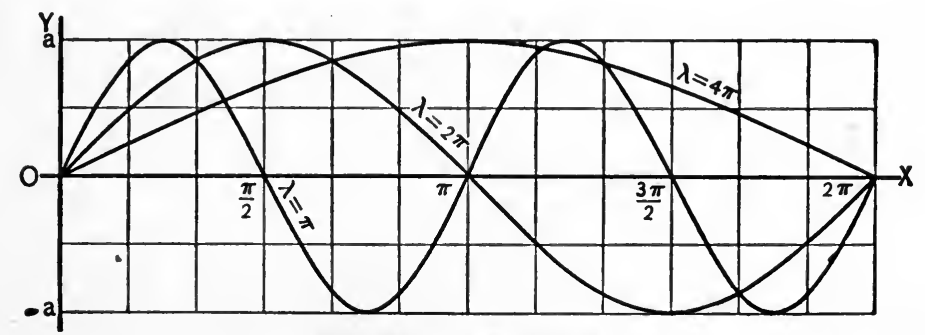

Fra. 54. $-y=a \sin k x$.

Figure 54 shows three sinusoids with wave-lengths $\pi, 2 \pi$, and $4 \pi$ respectively, the amplitudes being the same in each case. 
21.6. The Curve $y=a \sin (k x+\epsilon)$. This is a sine curve with an amplitude $a$ and which crosses the $x$-axis at the points where
$k x+\epsilon=0$,
$\pi$,
$2 \pi$,
$3 \pi$,
etc.,

that is, where

$$
x=-\epsilon / k, \quad(\pi-\epsilon) / k, \quad(2 \pi-\epsilon) / k, \quad(3 \pi-\epsilon) / k, \text { etc. }
$$

The distance between two consecutive points at which the curve crosses the $x$-axis in the same direction is $2 \pi / k$, the curve is therefore a sinusoid, with amplitude $a$ and wavelength $2 \pi / k$, but instead of passing through the origin it

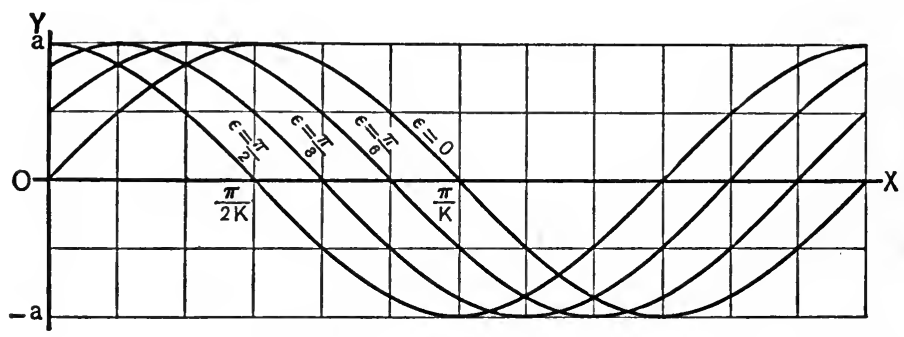

Fia. 55. $-\quad y=a \sin (k x+\epsilon)$.

crosses the $x$-axis a distance $-\epsilon / k$ from the origin. Figure 55 shows four sinusoids having equal amplitudes and wavelengths and $\epsilon=0, \pi / 6, \pi / 3, \pi / 2$, respectively.

In the case where $\epsilon=\pi / 2$ we have

$$
\begin{aligned}
y & =a \sin (k x+\pi / 2) \\
& =a \cos k x,
\end{aligned}
$$

which shows that the cosine curve, $y=a \cos k x$, is really a sinusoid having its highest point on the $y$-axis.

\subsection{Uniform Linear and Uniform Circular Motion of a} Point. By uniform linear motion is meant motion along a straight line with constant velocity, as that of a man walking 
at a steady gait on a sidewalk or of a train moving at a constant speed on a straight track. If a point moves from a fixed position, say the origin of coördinates, along some straight line, say the positive $x$-axis, at a speed of $v$ units per second, the equation of its motion is

$$
x=v t,
$$

where $t$ is the time in seconds since it left the origin, and $x$ the distance of the point from the origin.

Another simple motion is motion with a uniform angular velocity along the circumference of a circle as the motion of a point on the circumference of a fly-wheel or the end points of the hands of a clock. Such motion is called uniform circular motion. In figure 56 let $E$ represent the initial position

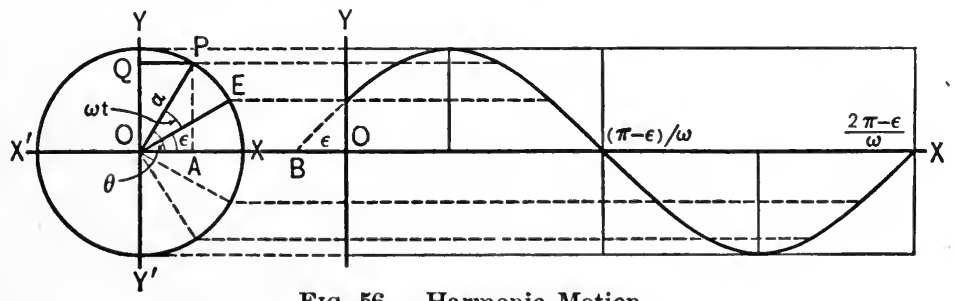

Fig. 56. - Harmonic Motion.

of the moving point corresponding to $t=0, P$ its position after $t$ seconds. If the angular velocity of the point is $\omega$ radians per second and $\theta$ the angle which the line $O P$ makes, the axis of reference $O X$, and $\epsilon$ the angle which $O E$ makes with this same axis of reference, then

$$
\theta=\omega t+\epsilon .
$$

This is the equation of uniform circular motion, for it determines the position of the point $P$ at any time $t$.

21.8. Simple Harmonic Motion. Let us next consider the motion of the point $Q$ which is the foot of the perpendicular from $P$ to the $y$-axis. 
While $P$ moves along the circumference of the circle, $Q$, moves along the straight line $Y Y^{\prime}$. $P$ has uniform velocity, but the velocity of $Q$ changes at every point, being greatest at $O$ and least at $Y$ and $Y^{\prime} . \quad P$ always moves forward along the circumference, while $Q$ moves to and fro along $Y Y^{\prime}$. Thus while the two points $P$ and $Q$ are definitely related, their motions are entirely different. The point $P$ moves with uniform circular motion; the point $Q$ is said to have simple harmonic motion along the straight line $Y Y^{\prime}$. If $P$ were a bright light looked at from a point in the plane of the circle at a considerable distance from its center, it would appear to have simple harmonic motion.

Denote $O Q$ by $y$, then since $O Q=A P=a \sin \theta=$ $a \sin (\omega t+\epsilon)$, we have

$$
y=a \sin (\omega t+\epsilon)
$$

which is the equation of simple harmonic motion along the $y$-axis.

21.9. Definitions. If a point moves along the circumference of a circle with uniform angular motion its projection along any diameter moves with simple harmonic motion.

The angle $\theta=\omega t+\epsilon$ is called the phase of the point $Q$ at the time $t$.

The distance $O Q=y$ is called the displacement of the point $Q$.

The angle $\epsilon$, which is the phase of $Q$ when $t=0$, is called the epoch of the simple harmonic motion.

$a=$ the radius of the circle, is called the amplitude of the s. h. m.

$T$, the time required for $P$ to complete one revolution, is called the period of the s.h.m. Since the velocity of $P$ is $\omega$ radians per second and the angle passed through in one complete revolution is $2 \pi$, we have

$$
\omega T=2 \pi \text {, from which } T=2 \pi / \omega, \omega=2 \pi / T \text {. }
$$


If we substitute this value of $\omega$ in the equation of s. h. m. we have

$$
y=a \sin \left(\frac{2 \pi t}{T}+\epsilon\right)
$$

where $T$ is the period of the oscillation.

Since $T$ is the time required to complete one revolution, $1 / T$ is the number of revolutions per second. The value of $1 / T$ is called the frequency of the oscillation. If we denote the frequency by $v$, the equation for $\mathrm{s} . \mathrm{h}$. $\mathrm{m}$. assumes the form

$$
y=a \sin (2 v \pi t+\epsilon) \text {. }
$$

Next to uniform linear motion and uniform circular motion, simple harmonic motion is most commonly met with in studying natural phenomena. The motion of the air particles in a sounding organ pipe, the vibrations of a tuning fork, the up and down motion in a water wave, the motion of an oscillating pendulum, of a galvanometer needle, of alternating electric current and of electro-magnetic waves, of light and sound waves, are all familiar examples of motions which approximate more or less closely simple harmonic motion.

21.10. Linear Harmonic Motion. Suppose now that a point has simple harmonic motion in a straight line while at the same time this straight line moves with uniform velocity in a direction at right angles to itself. The ordinate of the moving point then follows the law $y=a \sin (\omega t+\epsilon)$, while the abscissa of the same point follows the law $x=v t$. If we eliminate $t$ from these two equations we obtain for the equation of this new motion

$$
y=a \sin \left(\frac{\omega}{v} x+\epsilon\right)=a \sin (k x+\epsilon), k=\omega / v .
$$

The motion just described is called linear harmonic motion. It is the motion resulting from the composition of simple 
harmonic motion in a straight line with uniform linear motion at right angles to this straight line. The curve described by this motion is the sinusoid discussed in 21.6. Figure 55 shows this motion for the case when $v=1$. The curve is precisely that which would be obtained from the equation $y=a \sin (\omega t+\epsilon)$ by plotting the values of $t$ as abscissas and the corresponding values of $y$ as ordinates.

If the sine curve represents the flow of an alternating current, $\epsilon$ is called the lead or lag of the electric current according as $\epsilon$ is positive or negative. Two electric currents whose phases differ by $90^{\circ}$ are called "two phase" currents, three currents whose phases differ by $120^{\circ}$ are called "three phase" currents. Two or more currents which have the same phase are said to be "in phase."

Since linear harmonic motion is motion along a sinusoid, sinusoids are also called simple harmonic curves.

21.11. Compound Harmonic Curves. A compound harmonic curve is a curve whose equation is of the form

$$
y=a_{1} \sin \left(k_{1} x+\epsilon_{1}\right)+a_{2} \sin \left(k_{2} x+\epsilon_{2}\right)+a_{3} \sin \left(k_{3} x+\epsilon_{3}\right)+\text { etc. }
$$

Such a curve can be most readily constructed from the component curves, $y_{1}=a_{1} \sin \left(k_{1} x+\epsilon_{1}\right), y_{2}=a_{2} \sin \left(k_{2} x+\epsilon_{2}\right), y_{3}=$ $a_{3} \sin \left(k_{3} x+\epsilon_{3}\right)$, etc., for since

$$
y=y_{1}+y_{2}+y_{3}+\text { etc., }
$$

the ordinate of any point on the required curve corresponding to any given abscissa $x$ is merely the sum of the ordinates of the component curves corresponding to the same value of $x$.

Example. Plot the compound harmonic curve $y \doteq \sin x+\sin \left(\frac{3 x}{2}\right)$.

Solution. Plot separately the two curves

$$
y_{1}=\sin x, y_{2}=\sin \left(\frac{3 x}{2}\right), \text { (Fig. 57). }
$$

The required curve $y=y_{1}+y_{2}$ is then obtained by adding the two ordinates $y_{1}$ and $y_{2}$ corresponding to any given value of $x$. Thus if $x=$ 
$O A, y=A P=A P_{1}+A P_{2}$. It is important to keep in mind that for points below the $x$-axis the ordinates are negative. Thus if $x=O B, y=$ $B Q=B Q_{1}+B Q_{2}$, but $B Q_{2}$ is negative and numerically greater than $B Q_{1}$, so that their algebraic sum is negative. Again if $x=O C, y=C R$ $=C R_{1}+C R_{2}$, but $C R_{1}$ is negative and numerically less than $C R_{2}$, hence the algebraic sum of $C R_{1}$ and $C R_{2}$ is positive.

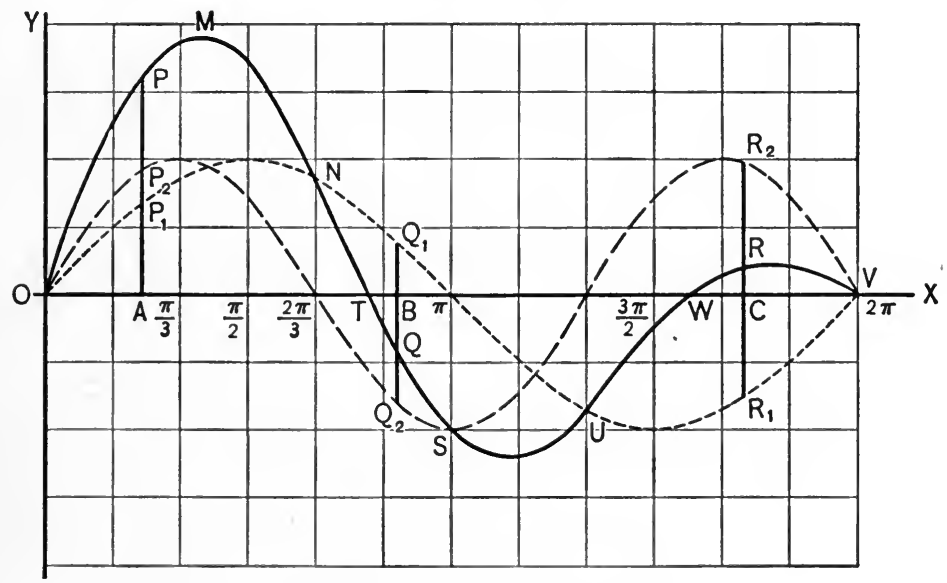

FIG. 57. $-y=\sin x+\sin \frac{3 x}{2}$.

It should be noted that certain points of the compound harmonic curve, as, for instance, the points $O, M, N, T, S, U, W, V$ (Fig. 57), may be located at sight, and such points generally suffice to make a rough sketch of the required curve.

If the compound harmonic curve is of the form $y=y_{1}-y_{2}$, the ordinate of the resultant curve is found by taking the difference of the corresponding ordinates of the component curves.

21.12. Fourier's Theorem. Fourier's Theorem states that: Any arbitrary periodic curve can be considered the resultant of a sum of simple harmonic curves, and can therefore be represented 
by an equation of the form

$$
y=a_{1} \sin \left(k_{1} x+\epsilon_{1}\right)+a_{2} \sin \left(k_{2} x+\epsilon_{2}\right)+\text { etc. }
$$

This is one of the most important theorems in modern analysis, but the proof is too difficult to be given in this place. Every periodic phenomenon whose changes can be measured may be represented by a periodic curve. Fourier's theorem shows how every such curve may be resolved into a series of simple harmonic curves.

\section{EXERCISE 21}

1. Construct the tangent curve, the cotangent curve, the secant curve, and the cosecant curve.

2. Sketch the curves $y=2 \sin \frac{x}{2}, y=\frac{1}{2} \sin (2 x)$ on the same sheet and then construct the curve

$$
y=2 \sin \frac{x}{2}+\frac{1}{2} \sin (2 x)
$$

3. Construct the curve $y=2 \sin \frac{x}{2}-\frac{1}{2} \sin (2 x)$.

4. Construct the two curves $y=\sin x+\cos x, y=\sin x-\cos x$.

5. By plotting the curves show that $y=\sin \left(x+90^{\circ}\right)$, $y=\sin \left(x-270^{\circ}\right)$, and $y=\cos x$ represent the same curve.

6. The distance of the piston of a steam engine from its extreme position corresponding to $\theta=0$ is given by the equation

$$
y=R\left[\frac{4 L+R}{4 L}-\frac{R}{4 L} \cos (2 \theta)-\cos \theta\right],
$$

where $R$ is the length of the crank arm, $L$ the length of the connecting rod, and $\theta$ the angle which the crank arm makes with a fixed direction. Plot the curve which will give $y$ for 
any value of $\theta$ from $0^{\circ}$ to $360^{\circ}$, for the case when $R=10$, $L=25$.

7. Wrap a strip of paper around a cylindrical piece of wood like a pencil and then cut the wrapped stick in two by cutting it obliquely with a fine saw. By unwrapping the paper its cut edge will form a sine curve. Prove it. 


\section{LESSON XXII — SOLUTION OF RIGHT TRIANGLES}

22.1. The Parts of a Triangle. Every triangle has six parts, three sides and three angles. When three of these parts, including one side, are known, the remaining three may be found. Every right triangle has one right angle; there remain then only five other parts, and if two of these, including a side, are known, the remaining three may be found. The process of finding these parts by computation is known as solving the triangle. The solution, as we shall see, is most readily effected by aid of the trigonometric functions.

22.2. How to Find the Functions of a Given Angle. When an angle is given, any desired function of that angle may be found immediately from a natural functions table. Thus if $A=15^{\circ} 20^{\prime}$, we find (Macmillan Table, p. 29)

$$
\begin{aligned}
\sin A=0.26433, & \cos A=0.96440, \tan A=0.27419, \\
\cot A & =3.6470 .
\end{aligned}
$$

If the angle is $15^{\circ} 20^{\prime} 25^{\prime \prime}$ and we wish to find any function, let us say the sine, we proceed as follows :

$\sin 15^{\circ} 20^{\prime}=0.26433, \sin 15^{\circ} 21^{\prime}=0.26471$, from the tables.

To find $\sin 15^{\circ} 20^{\prime} 25^{\prime \prime}$ we must increase 0.26433 by $\frac{25}{60}$ of the difference between 0.26471 and 0.26433 , which is $\frac{25}{60}$ of 0.00038 , or 0.00016 . Hence

$$
\sin 15^{\circ} 20^{\prime} 25^{\prime \prime}=0.26433+0.00016=0.26449 \text {. }
$$

Similarly we have,

$\cos 15^{\circ} 20^{\prime}=0.96440, \cos 15^{\circ} 21^{\prime}=0.96433$, from the tables.

To find $\cos 15^{\circ} 20^{\prime} 25^{\prime \prime}$ we must diminish 0.96440 by $\frac{25}{60}$ 
of the difference between these two numbers or by $\frac{25}{60}$ of 0.00007 , which is 0.00003 ; hence

$$
\cos 15^{\circ} 20^{\prime} 25^{\prime \prime}=0.96440-0.00003=0.96437 \text {. }
$$

If the angle is greater than $45^{\circ}$ we must look for the number of the degrees at the bottom of the page in the tables and for the minutes in the right-hand column, otherwise the work is the same.

\subsection{How to Find the Angle Corresponding to a Given} Function. The table also enables us to find the angle provided we know one of the functions of the angle. Suppose the tangent of an unknown angle is 0.75858. Turning to page 40 of the tables we find this very number in the column marked tangent and headed by $37^{\circ}$ and in the line which has $11^{\prime}$ written to the left. The required angle is therefore $37^{\circ} 11^{\prime}$.

More frequently, however, the exact number will not be found in the table. Suppose, for instance, that we wish to know the angle whose tangent is 0.75877 . This number is not given in the table. The number next smaller which is given in the tangent column is 0.75858 , which is the tangent of $37^{\circ} 11^{\prime}$; the number next larger is 0.75904 , which is the tangent of $37^{\circ} 12^{\prime}$. The difference of these two numbers is 0.00046 , the difference between 0.75858 and 0.75877 is 0.00019 , hence we must increase $37^{\circ} 11^{\prime}$ by $\frac{0.00019}{0.00046}$ of $1^{\prime}$ or $60^{\prime \prime}$, that is, by $\frac{19}{4} \frac{9}{6}$ of $60^{\prime \prime}$, or $25^{\prime \prime}$. The required angle is therefore $37^{\circ} 11^{\prime} 25^{\prime \prime}$.

22.4. Interpolation. The process which we have just employed in finding the angle corresponding to a function which is not directly found in the table is called interpolation. It is based upon an assumption known as the Principle of Proportional Parts, which is 
For small changes in the angle the change in the function is proportional to the change in the angle. This principle is not exactly true, but the results based on this assumption are sufficiently exact for most practical purposes.

22.5. Secants and Cosecants. Most tables do not give secants and cosecants. These when needed may be found from the cosines and sines respectively.

Thus

$$
\begin{aligned}
& \sec 15^{\circ} 20^{\prime}=1 / \cos 15^{\circ} 20^{\prime}=1 / 0.96440=1.03691 ; \\
& \csc 15^{\circ} 20^{\prime}=1 / \sin 15^{\circ} 20^{\prime}=1 / 0.26443=3.78162 .
\end{aligned}
$$

22.6. Solution of Right Triangles. Let $A B C$ (fig. 58) be any right triangle, $A, B, C$, the angles, and $a, b, c$, the sides

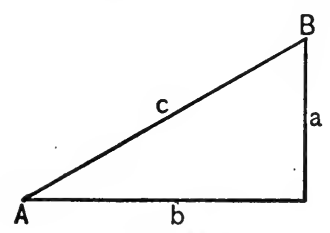

FIG. 58. opposite these angles. Then

$$
\begin{aligned}
a / c & =\sin A=\cos B, \\
b / c & =\cos A=\sin B, \\
a / b=\tan A & =\cot B .
\end{aligned}
$$

These equations enable us to solve any right triangle whatsoever, for no matter which two parts are given (at least one being a side), some one of these equations will give a third part, and then this third part combined with one of the other parts will give a fourth, and so on. Thus suppose that $A$ and $c$ are given. Then the first equation gives $a$, the second gives $b$, and of course $B=90^{\circ}-A$. Suppose that $a$ and $c$ are given. Then the first equation gives $\sin A$, from which $A$ is found by means of the table. Then $A$ being known, the second equation gives the value of $b$, and as before $B=90^{\circ}-A$. Suppose that $a$ and $b$ are given. Then the third equation gives $\tan A$, from which $A$ is found by means of the table. Then $A$ being known, the first equation gives $c$, and $B=90^{\circ}-A$. 
22.7. General Rule for Solving Right Triangles. Which one of the above equations is to be used depends on the parts of the triangle which are given, and the part that is to be found. The general rule to be observed is :

Use that function of the angle which involves the two given parts. If the given parts are two sides, the resulting formula will give an angle; if one of the given parts is an angle, the resulting formula will give a side.

22.8. Numerical Examples. Example ( $a$ ). Given one angle of a right triangle, $A=36^{\circ} 40^{\prime}$, and the hypotenuse, $c=3.125$; to solve the triangle.

Solution.

1. To find $B . \quad B=90^{\circ}-A=90^{\circ}-36^{\circ} 40^{\prime}$ $=53^{\circ}{ }^{\prime} 0^{\prime}$.

2. To find $a . \quad a / c=\sin A$, or $a=c \sin A$. From the tables $\sin A=0.59716$, hence $a=3.125 \times 0.59716=1.866$.

3. To find $b . \quad b / c=\cos A$, or $b=c \cos A$.

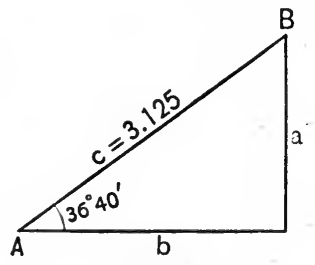

Fig. 59.

From the tables $\cos A=0.80212$,

hence $b=3.125 \times 0.80212=2.507$.

Example (b). Given one side of a right triangle, $a=45.425$, and the hypotenuse, $c=75.48$; to solve the triangle.

Solution. 1. To find $A$. $\sin A=\frac{a}{c}=\frac{45.425}{75.48}=0.60182$.

From the tables $A=\mathbf{3 7}^{\circ} \mathbf{0 0}$ '.

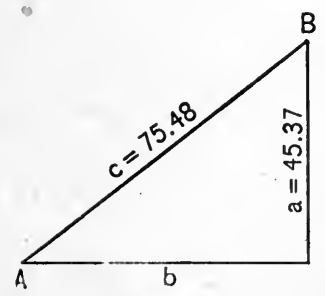

Fia. 60.

2. To find $B$. $B=90^{\circ}-A=90^{\circ}-37^{\circ}=53^{\circ}$.

3. To find $b . \quad b / c=\cos A$, or $b=c \cos A$.

From the tables $\cos A=\mathbf{0 . 7 9 8 6 4}$, hence $b=75.48 \times 0.79864=60.28$.

Example (c). Given one angle of a right triangle, $B=42^{\circ} 15^{\prime}$, and one side $a=513.9$; to solve the triangle.

Solution. 1. To find $A . A=90^{\circ}-B=$ $90^{\circ}-42^{\circ} 15^{\prime}=47^{\circ} 45^{\prime}$. 
2. To find $c . \quad a / c=\cos B$, or $c=a / \cos B$.

From the tables $\cos B=0.74022$,

hence $c=513.9 / 0.74022=694.2$.

3. To find $b . \quad b / a=\tan B$, or $b=a \tan B$.

From the tables $\tan B=0.90834$,

hence $b=513.9 \times 0.90834=466.8$.

22.9. Applied Problems Involving Right Triangles. The knowledge of solving right triangles may be applied to the solution of a great variety of problems in geometry, physics, geography, surveying, engineering, navigation, meteorology, and astronomy. In fact there is scarcely a science or an art in which there is not an occasional use for the solution of triangles.

In every given problem a figure should be drawn showing plainly the parts of the triangle that are given and the part or parts that are to be found. When that has been done the solution proceeds as has been shown in 22.8.

Example. To find the width of a river a distance $C A=500 \mathrm{ft}$. was measured off along the bank of the river and the angle $C A B=36^{\circ} 15^{\prime}$ was measured which a rock $B$ on the opposite shore of the river directly

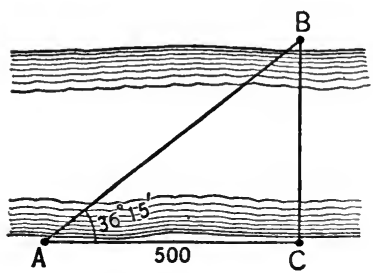

Fig. 61. opposite $C$ made with the direction of $A C$. Required the width of the river.

Solution. The width of the river is represented by the line $C B$ in figure 61 . Now $B C / A C=\tan A$, or $B C=A C \times$ $\tan A$.

From the tables we find

$$
\tan A=\tan 36^{\circ} 15^{\prime}=0.73323 \text {, }
$$

hence $B C=500 \mathrm{ft} . \times 0.73323=367 \mathrm{ft}$.

22.10. Number of Significant Figures. When tables are used in computation the results need not be carried out to a greater number of figures than that given in the table. When a five-place table is used, no more than five correct significant 
figures can be obtained and even the fifth figure can not be relied on with certainty.

When the given numbers of a problem are the results of measurement, the answers need not contain more significant figures than the least accurate of the given numbers. Thus, if one side of a triangle is measured to the nearest inch and another to the nearest tenth of an inch, the third side, if found by computation, needs only to be given to the nearest inch, for plainly, an uncertainty in any one of the given numbers will cause a corresponding uncertainty in the answer. .

When a problem involves both distances and angles, the following rules will give some guidance as to comparable accuracies :

Distances expressed in three figures call for angles expressed to the nearest minute.

Distances expressed in four figures call for angles expressed to the nearest tenth of a minute.

Distances expressed in five figures call for angles expressed to the nearest second.

Distances expressed in six figures call for angles expressed to the nearest tenth of a second. A six-place table must be used to obtain like accuracy in the answers.

Distances expressed in seven figures call for angles expressed to the nearest hundredth of a second. A seven-place table must be used to obtain like accuracy in the answers.

\section{EXERCISE $22(\boldsymbol{A})$}

Solve the following right triangles :

Given Pakts

Required Parts
1. $A=25^{\circ} 15^{\prime}$,
$c=45.6$
$a=19.45, \quad b=41.24$.
2. $b=0.2345$,
$a=27.345$;
$B=$
$c=$
3. $A=56^{\circ} 27^{\prime} 36^{\prime \prime}, a=27.345$;
$b=18.127, c=32.807$. 
4. From a point $200 \mathrm{ft}$. from the foot of a wireless telegraph pole the top of the pole was found to form an angle of $53^{\circ} 45^{\prime}$. Find the height of the pole.

5. A branch railroad is to be constructed from a point $A$ to a second point $B$ which is 6.27 miles east and 10.15 miles north of the first. What will be the direction of the branch road, assuming that it follows a straight line? Ans. N. $58^{\circ} 17.6^{\prime} \mathrm{E}$.

6. The summit of a mountain known to be $12,560 \mathrm{ft}$. high is seen at an angle of elevation of $25^{\circ} 36^{\prime}$ from a camp located at an altitude of $5940 \mathrm{ft}$. Compute the air-line distance from the camp to the summit.

7. On a map on which one inch, represents $1000 \mathrm{ft}$. contour lines are drawn for differences of $100 \mathrm{ft}$. in altitude. What is the inclination of the surface represented by that portion of the map at which the contour lines are one-eighth of an inch apart?

Ans. $38^{\circ} 40^{\prime}$.

8. Through what angle must a round column of stone three feet in diameter and ten feet long be tilted before it begins to fall?

9. A winding stair with treads $6 \mathrm{ft}$. long makes five complete turns in rising $50 \mathrm{ft}$. At what angle does a person ascend who walks up the outer edge of the stairs.

Ans. $12^{\circ} 27.6^{\prime}$.

10. When the sun's altitude is $40^{\circ}$ a factory chimney casts a shadow $225 \mathrm{ft}$. long. Find the height of the chimney.

11. Positions on the map of France are given by their coördinates, the abscissas being distances east of a fixed north and south line, the ordinates distances north of a fixed east and west line. If the coördinates of a target are $(6893 \mathrm{~m}$., $12,180 \mathrm{~m}$.) and the coördinates of the position of the gun are $(12,862 \mathrm{~m} ., 5638 \mathrm{~m}$.$) , draw a figure and find the range and$ 
direction of the target from the gun. (It is assumed that the target is invisible from the position of the gun.)

\section{EXERCISE $22(B)$}

Given PARTs

1. $A=54^{\circ} 32^{\prime}, c=65.4$;

2. $a=123.7, A=37^{\circ} 47.8^{\prime}$;

3. $b=4237.2, A=27^{\circ} 56^{\prime} 10^{\prime \prime}$;
Required Parts

$a=53.3, \quad b=37.9$.

$b=\quad c=$

$a=2246.9, c=4796.1$.

In the following problems the distances are considered straight lines, not ares of circles. A knot or sea mile is the length of a minute of the arc on the earth's surface or approximately one and one-sixth common miles. The Mariner's Compass shown in figure 62 has 32 divisions or points, each point being the 32nd part of $360^{\circ}$, or $11^{\circ} 15^{\prime}$. In sailing a course, the east and west component of the course is called its departure, the north and south component its dif-

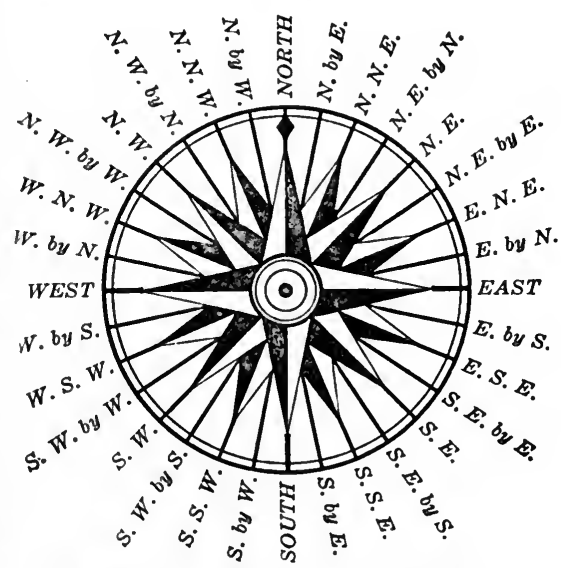

- Fig. 62. - Mariner's Compass. ference in latitude. The difference in latitude is usually expressed in degrees and minutes $\left(1\right.$ mile $\left.=1^{\prime}\right)$.

4. A ship sails N.E. by N. a distance of 300 miles. Find its departure.

5. A cruiser sails S.E. by E. at the rate of 15 knots per hour. At what rate is the cruiser moving due S.? Due E.? Ans. $8.33 ; 12.47$. 
6. A ship sails from latitude $27^{\circ} 24^{\prime}$ N. on a course N.N.E. a distance of 475 miles. What was its new latitude and departure?

7. From a "crow's nest" $106 \mathrm{ft}$. above the water the angle of depression of a rock on the beach is $10^{\circ} 30^{\prime}$. Find the distance of the rock from the foot of the mast. Ans. $572 \mathrm{ft}$.

8. A battle cruiser sailing S.W. at a rate of 18 knots observes at 10.15 A.M. a fort bearing S.S.E. Thirty minutes later the fort is seen due S.E. Find the distance of the cruiser from the fort at the time of each observation.

9. A ship bearing E. by S. and making 15 knots per hour had a current setting starboard broadside against her track which after 6 hours sailing brought her to a point known to be 125 miles from her starting point. What was the true course of the ship? Ans. S. $34^{\circ} 48^{\prime} \mathrm{E}$.

10. An observer on board ship finds the time between the flash of a gun from a fort located S.W. by W. and the report of the gun to be 7 seconds. After awhile the gun was heard again and this time the time between the flash and the report was 12 seconds. The course of the ship lay S.E. by S. Find the bearing and distance of the fort from the second position of the ship. (Assume the velocity of sound as $1090 \mathrm{ft}$. per second.) 


\section{LESSON XXIII-ARITHMETIC SOLUTION OF OBLIQUE}

TRIANGLES

23.1. Every Triangle Is the Sum or Difference of Two Right Triangles. We have learned how any right triangle may be solved with the aid of a table of natural functions. Now every other triangle may be looked upon as the sum or difference of two right triangles, formed by drawing a perpendicular from either of the three vertices to the side opposite. This suggests the possibility of solving any triangle by the method explained in the last lesson. If the student has the time to pursue this suggestion he will find that he is able, without much, if any, difficulty to solve any given triangle. However, the method is somewhat roundabout and is therefore not employed by practical computers.

23.2. The Four Cases of Oblique Triangles. In studying the shorter methods of solving oblique triangles it is best to consider separately the different cases that may arise. There are four cases and no more, namely :

Case I. Given one side and any two angles, as $a, B$, and $C$. If the given angles are $A$ and $C$ or $A$ and $B$, the third angle $B$ or $C$ may at once be found from the fact that three angles of any triangle are together equal to $180^{\circ}$.

Case II. Given two sides and an angle opposite one of them, as $a, b$, and $A$.

Case III. Given two sides and the included angle, as $a, b$, and $C$.

Case IV. Given the three sides, $a, b$, and $c$. 
When the three angles, $A, B$, and $C$, are given the triangle can not be solved, for we may construct as many different triangles as we please having the three given angles for angles. All such triangles are similar. The angles being given, we may compute the ratios between the sides but not the length of the sides.

23.3. The Law of Sines. In order to facilitate the solution of oblique triangles we need two preliminary theorems.

Let $A B C$ represent any plane triangle. From the vertex $C$ draw the perpendicular $C D=p$ to the opposite side $A B$ (fig. 63) or the opposite side prolonged (fig. 64). We then have

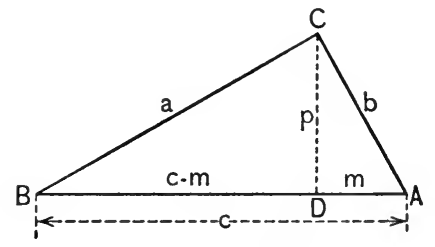

Fig. 63.

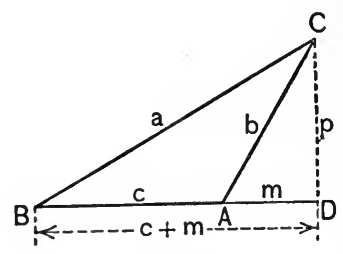

Fig. 64.

in the right triangle $B C D$

$$
p=a \sin B,
$$

and in the right triangle $A C D$

(fig. 63)

$$
p=b \sin A
$$

(fig. 64)

$$
\begin{aligned}
p & =b \sin \left(180^{\circ}-A\right) \\
& =b \sin A
\end{aligned}
$$

Hence whether the triangle is acute or obtuse we have in either case

from which

$$
p=a \sin B=b \sin A,
$$

$$
\frac{a}{\sin A}=\frac{b}{\sin B} .
$$


This proportion is known as the Law of Sines. $a$ and $b$ are any two sides of the triangle. The Law of Sines therefore may be stated

$$
\frac{a}{\sin A}=\frac{b}{\sin B}=\frac{c}{\sin C}
$$

in words: In any triangle, the sides are proportional to the sines of the opposite angles.

23.4. The Law of Cosines. Again, we have

in the right triangle $B C D$

(fig. 63)

$$
\begin{aligned}
a^{2} & =(c-m)^{2}+p^{2} \\
& =c^{2}-2 c m+m^{2}+p^{2},
\end{aligned}
$$

(fig. 64)

$$
\begin{aligned}
a^{2} & =(c+m)^{2}+p^{2} \\
& =c^{2}+2 c m+m^{2}+p^{2},
\end{aligned}
$$

but in the right triangle $A C D$

$$
m^{2}+p^{2}=b^{2},
$$

hence

$$
\begin{aligned}
a^{2} & =c^{2}+b^{2}-2 c m \\
& =b^{2}+c^{2}-2 b c \cos A,
\end{aligned}
$$

since

$$
m=b \cos A .
$$

$$
\begin{aligned}
a^{2} & =c^{2}+b^{2}+2 c m \\
& =b^{2}+c^{2}-2 b c \cos A,
\end{aligned}
$$

$$
\begin{aligned}
m & =b \cos \left(180^{\circ}-A\right) \\
& =-b \cos A .
\end{aligned}
$$

We see that whether the angle opposite $a$ is acute or obtuse we have in either case

$$
a^{2}=b^{2}+c^{2}-2 b c \cos A
$$

and similarly

$$
\begin{aligned}
& b^{2}=c^{2}+a^{2}-2 a c \cos B, \\
& c^{2}=a^{2}+b^{2}-2 a b \cos C .
\end{aligned}
$$

This is the Law of Cosines. In words:

In any triangle, the square on any sirle is equal to the sum of the squares on the other two sides diminished by twice the product of these two sides times the cosine of the included angle. 
23.5. The Angles in Terms of the Sides. By solving the formulas of the preceding section for $\cos A, \cos B$, and $\cos C$ we find

$\cos A=\frac{b^{2}+c^{2}-a^{2}}{2 b c}, \quad \cos B=\frac{c^{2}+a^{2}-b^{2}}{2 c a}, \quad \cos C=\frac{a^{2}+b^{2}-c^{2}}{2 a b}$, from which the angles may be found when the sides are given.

23.6. Application of Law of the Sines. The law of sines is used in solving the first case and the second case of triangles. How this is done will be sufficiently clear by following through an example under each case.

Case I. Example. Given $a=13, B=63^{\circ}, C=44^{\circ}$; to find the other parts of the triangle.

Solution. $A=180^{\circ}-(B+C)=180^{\circ}-\left(63^{\circ}+44^{\circ}\right)=180^{\circ}-107^{\circ}=7^{\circ}$.

By the law of sines,

$$
\begin{aligned}
& b=a \sin B / \sin A=13 \times 0.89101 / 0.95630=\mathbf{1 2 . 1}, \\
& c=a \sin C / \sin A=13 \times 0.69466 / 0.95630=\mathbf{9 . 4} .
\end{aligned}
$$

Case II. Example. Given $a=47.3, b=70.5, A=32^{\circ} 50^{\prime}$; to find the other parts.

Solution. A rough sketch of the triangle as in figure 65 shows that there are two triangles, $A B C$ and $A B^{\prime} C$, which satisfy the given conditions.

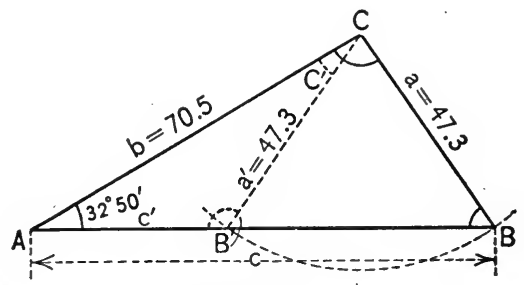

Fig. 65 .

By the law of sines

$$
\sin B=\frac{b \sin A}{a}=\frac{70.5 \times 0.54220}{47.3}=0.80814,
$$

from which

$$
B=53^{\circ} 55^{\prime}, B^{\prime}=180^{\circ}-53^{\circ} 55^{\prime}=126^{\circ} 05^{\prime},
$$

and

$$
\begin{aligned}
& C=180^{\circ}-(A+B)=180^{\circ}-86^{\circ} 45^{\prime}=\mathbf{9 3}^{\circ} \mathbf{1 5}^{\prime}, \\
& C^{\prime}=180^{\circ}-\left(A+B^{\prime}\right)=180^{\circ}-158^{\circ} 55^{\prime}=\mathbf{2 1}^{\circ} 05^{\prime} .
\end{aligned}
$$


To find $c$ and $c^{\prime}$ we again use the law of sines,

$$
\begin{aligned}
& c=a \sin C / \sin A=47.3 \times 0.99839 / 0.54220=\mathbf{8 7 . 1}, \\
& c^{\prime}=a \sin C^{\prime} / \sin A=47.3 \times 0.35973 / 0.54220=\mathbf{3 1 . 4} .
\end{aligned}
$$

The required solutions are therefore,

or

$$
\begin{aligned}
B & =53^{\circ} 55^{\prime}, \quad C=93^{\circ} 15^{\prime}, \quad c=87.1 ; \\
B^{\prime} & =126^{\circ} 05^{\prime}, \quad C^{\prime}=21^{\circ} 05^{\prime}, \quad c^{\prime}=31.4 .
\end{aligned}
$$

23.7. Application of the Law of Cosines. The solution of both the third and fourth case of triangles is rendered very simple by using the law of cosines, as will be seen from the examples which follow.

Case III. Example. Given $a=25.9, b=35.6, C=15^{\circ} 24^{\prime}$; to find the side $c$.

Solution. By the law of cosines,

$$
\begin{aligned}
c^{2}=a^{2}+b^{2}-2 b c \cos C & =(25.9)^{2}+(35.6)^{2}-2 \times 25.9 \times 35.6 \times 0.96410 \\
& =670.81+1267.36-1777.88=160.29 \\
c & =\sqrt{160.29}=\mathbf{1 2 . 6 6} .
\end{aligned}
$$

Case IV. Example. Given $a=6, b=7, c=8$; to find the angles of the triangle.

Solution. By the law of cosines (23.5),

$$
\begin{aligned}
\cos A=\left(b^{2}+c^{2}-a^{2}\right) / 2 b c & =\left(7^{2}+8^{2}-6^{2}\right) / 112=(49+64-36) / 112 \\
& =77 / 112=0.68750, \\
\cos B=\left(c^{2}+a^{2}-b^{2}\right) / 2 c a & =\left(8^{2}+6^{2}-7^{2}\right) / 96=(64+36-49) / 96 \\
& =51 / 96=0.53125,
\end{aligned}
$$

$\cos C=\left(a^{2}+b^{2}-c^{2}\right) / 2 a b=\left(6^{2}+7^{2}-8^{2}\right) / 84=(36+49-64) / 84$

$$
=21 / 84=0.25000 \text {. }
$$

Hence from the tables $A=46^{\circ} 34^{\prime}, B=57^{\circ} 55^{\prime}, C=75^{\circ} 31^{\prime}$.

23.8. The Area of Any Triangle in Terms of the Sides. Since the area of any triangle is equal to one-half the product of the base by the altitude, and since in either figure 63 or figure 64 the altitude $p=b \sin A$, we have, calling the area of angle $T, T=\frac{1}{2} b c \sin A$.

Now $\sin ^{2} A=1-\cos ^{2} A$, and $\cos ^{2} A=\left(\frac{b^{2}+c^{2}-a^{2}}{2 b c}\right)^{2}$, 
hence $\sin ^{2} A=1-\left(\frac{b^{2}+c^{2}-a^{2}}{2 b c}\right)^{2}=\frac{(2 b c)^{2}-\left(b^{2}+c^{2}-a^{2}\right)^{2}}{4 b^{2} c^{2}}$

$$
\begin{aligned}
& =\frac{\left(2 b c+\overline{b^{2}+c^{2}-a^{2}}\right)\left(2 b c-\overline{b^{2}+c^{2}-a^{2}}\right)}{4 b^{2} c^{2}} \\
& =\frac{\left(b^{2}+2 b c+c^{2}-a^{2}\right)\left(a^{2}-\overline{\left.b^{2}-2 b c+c^{2}\right)}\right.}{4 b^{2} c^{2}} \\
& =\frac{\left(\overline{b+c^{2}}-a^{2}\right)\left(a^{2}-\overline{b-c^{2}}\right)}{4 b^{2} c^{2}} \\
& =\frac{(b+c+a)(b+c-a)(a+b-c)(a-b+c)}{4 b^{2} c^{2}} .
\end{aligned}
$$

Let us put $a+b+c=2 s$, so that $s$ denotes half the sum of three sides, then

$$
b+c-a=2(s-a), a-b+c=2(s-b), \text { and }
$$

$\sin ^{2} A=\frac{2 s \cdot 2(s-a) \cdot 2(s-b) \cdot 2(s-c)}{4 b^{2} c^{2}}=\frac{4 s(s-a)(s-b)(s-c)}{b^{2} c^{2}}$

and

$$
\boldsymbol{T}=\frac{1}{2} b c \sin A=\sqrt{s(s-a)(s-b)(s-c)} .
$$

Both of these formulas are exceedingly useful. The first gives the area of a triangle in terms of two sides and the included angle, the second in terms of the three sides.

Example. Given $a=6, b=7, c=8$; to find the area of the triangle.

Solution. $\quad 2 s=a+b+c=6+7+8=21 ; s=10.5$;

$$
\begin{aligned}
& s-a=10.5-6=4.5 ; \\
& s-b=10.5-7=3.5 ; \\
& s-c=10.5-8=2.5 ;
\end{aligned}
$$

hence

$$
\begin{aligned}
T=\sqrt{s(s-a)(s-b)(s-c)} & =\sqrt{10.5 \times 4.5 \times 3.5 \times 2.5} \\
& =\sqrt{413.44}=\mathbf{2 0 . 3 3} .
\end{aligned}
$$

\section{EXERCISE 23}

1. The angles of elevation of a captive balloon as seen by two observers on opposite sides of it and $1000 \mathrm{ft}$. apart are $40^{\circ} 12^{\prime}$ and $46^{\circ} 36^{\prime}$ respectively. How far is the balloon from each of the observers? Ans. $728 \mathrm{ft}$; $646 \mathrm{ft}$. 
2. Two railroads intersecting at an angle of $42^{\circ} 48^{\prime}$ cross the same river, the first at a distance of 2.9 miles, the second at a distance of 4.5 miles from their point of intersection. Find the distance between the points where the roads cross the river. Ans. 3.1 miles.

3. The distances between three ports $\mathrm{A}, \mathrm{B}, \mathrm{C}$ are as follows : $A B=75$ miles, $B C=30$ miles, $C A=62$ miles. $\mathrm{C}$ is due north of $\mathrm{A}$. In what direction from $\mathrm{A}$ is $\mathrm{B}$ ?

4. Two highways meet at an angle of $30^{\circ}$. From a town $\mathbf{A}$ on the one highway, a branch road 5 miles long runs to a town $\mathrm{B}$, located on the other highway. If $\mathrm{A}$ is 6 miles from $\mathrm{C}$, the intersection of the two highways, how far is $\mathrm{B}$ from $\mathrm{C}$ ?

Ans. $1.2 \mathrm{mi}$., or $9.2 \mathrm{mi}$.

5. The sewer assessment of a certain city, improvement district is 2.13 cents per square foot. What is the total assessment on a triangular lot whose sides measure $150 \mathrm{ft}$., $120 \mathrm{ft}$., and $80 \mathrm{ft}$., respectively?

Ans. $\$ 101.84$.

6. Two stations, $\mathrm{A}$ and $\mathrm{B}$, on opposite sides of a mountain are both visible from a third station $\mathrm{C}$. $A C$ is 12.7 miles, $C B$ is 15.6 miles, and the angle $A C B$ is $47^{\circ} 35^{\prime}$. Find the distance from $\mathrm{A}$ to $\mathrm{B}$.

7. A fort as observed from board ship bears N. by E. After the ship has sailed 12 miles N.W. the fort bears exactly east. How far is the fort from the second position of the ship?

8. A launch leaves the shore while the tide is running from west to east, and after 3 hours reaches an island 31 miles in the direction $\mathrm{N}$. by $\mathrm{E}$. from the starting point. Assuming that the launch sailed a straight course and that its speed in still water is $10 \frac{1}{4}$ miles per hour, what was the velocity of the tide? (Two answers.)

Ans. 3.55 miles or 0.48 mile per hour. 
LESSON XXIV - FUNCTIONS OF THE SUM AND DIFFERENCE OF 'TWO ANGLES

24.1. Addition and Subtraction Theorems. It is frequently necessary to express a function of the sum or difference of two angles in terms of the functions of the separate angles. This is done by means of certain theorems known as addition and subtraction theorems. The principal ones of these theorems are stated below and should be memorized once for all by the student. Their proofs will be given in the following sections.

$$
\begin{gathered}
\text { Addition Theorems } \\
\sin (x+y)=\sin x \cos y+\cos x \sin y . \\
\cos (x+y)=\cos x \cos y-\sin x \sin y . \\
\tan (x+y)=(\tan x+\tan y) /(1-\tan x \tan y) . \\
\text { Subtraction Theorems } \\
\sin (x-y)=\sin x \cos y-\cos x \sin y . \\
\cos (x-y)=\cos x \cos y+\sin x \sin y . \\
\tan (x-y)=(\tan x-\tan y) /(1+\tan x \tan y) .
\end{gathered}
$$

24.2. The Sine of the Sum or Difference of Two Angles. Place the two angles $x$ and $y$ side by side so that they have a common vertex and so that the initial side of the angle $y$ coincides with the terminal side of the angle $x$ (fig. 66). From any point $P$ in the side common to the two angles draw a perpendicular to $O P$ and denote the points in which this perpendicular intersects the other two sides by $R$ and $S$. If 
we represent the triangles $R O S, R O P$, and $P O S$ by $T_{x+y}, T_{x}$, and $T_{y}$ respectively, we have by 23.8 ,

$$
\begin{aligned}
2 \cdot T_{x+y} & =O R \cdot O S \cdot \sin (x+y), \\
2 \cdot T_{x} & =O R \cdot O P \cdot \sin x, \\
2 \cdot T_{y} & =O P \cdot O S \cdot \sin y,
\end{aligned}
$$

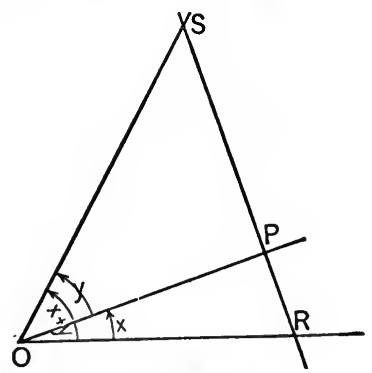

Frg. 66.

and therefore, since $T_{z+y}=T_{x}+T_{y}$,

$$
O R \cdot O S \cdot \sin (x+y)=O R \cdot O P \cdot \sin x+O P \cdot O S \cdot \sin y \cdot
$$

Let us divide both sides of this equation by $O R \cdot O S$, then

$$
\sin (x+y)=\frac{O P}{O S} \sin x+\frac{O P}{O R} \sin y \text {. }
$$

But from the figure we see that

hence

$$
O P / O S=\cos y \text {, and } O P / O R=\cos x,
$$

$$
\sin (x+y)=\sin x \cos y+\cos x \sin y .
$$

In our figure the sum of the angles $x$ and $y$ is less than $180^{\circ}$, but the proof holds true when the sum of $x$ and $y$ is greater than $180^{\circ}$ and when either $x$ or $y$ or both are negative, provided proper attention is given to the signs of the areas, which must be considered negative if the sines of the corresponding angles are negative. The student should repeat the proof for a case when $y$ is negative, and again when $x+y$ is greater than $180^{\circ}$. 
The proof fails when the perpendicular through $P$ does not meet both $O A$ and $O C$, that is, when one of the angles $x$ or $y$ is a right angle. In that case the theorem can be verified directly, for suppose $\cdot y=90^{\circ}$, then

$$
\begin{aligned}
\sin (x+y) & =\sin \left(x+90^{\circ}\right)=\sin 90^{\circ} \cos x+\cos 90^{\circ} \sin x \\
& =1 \cos x+0 \sin x=\cos x,
\end{aligned}
$$

which is true by Exercise 19.3 (a).

Since the addition formula for the sine holds true for all values of the angles, we may change the sign of $y$. This gives us

$$
\begin{aligned}
\sin (x-y) & =\sin x \cos (-y)+\cos x \sin (-y) \\
& =\sin x \cos y-\cos x \sin y,
\end{aligned}
$$

since by $19.6 \quad \cos (-y)=\cos y$, and $\sin (-y)=-\sin y$.

This proves the subtraction theorem for the sine.

\subsection{The Cosine of the Sum or Difference of Two Angles.}

By $19.7 \quad \cos (x+y)=\sin \left(90^{\circ}-\overline{x+y}\right)=\sin \left(\overline{90^{\circ}-x}-y\right)$ and by the subtraction theorem for the sine we have

$$
\begin{aligned}
\sin \left(\overline{90^{\circ}-x}-y\right) & =\sin \left(90^{\circ}-x\right) \cos y-\cos \left(90^{\circ}-x\right) \sin y \\
& =\cos x \cos y-\sin x \sin y
\end{aligned}
$$

which proves the addition theorem for the cosine.

If in the addition theorem for the cosine we change the sign of $y$ we obtain

$$
\begin{aligned}
\cos (x-y) & =\cos x \cos (-y)-\sin x \sin (-y) \\
& =\cos x \cos y+\sin x \sin y,
\end{aligned}
$$

which is the subtraction theorem for the cosine.

24.4. The Tangent of the Sum and Difference of Two Angles. Since the tangent of an angle is equal to the sine of the angle divided by the cosine of the angle, we have

$$
\tan (x+y)=\frac{\sin (x+y)}{\cos (x+y)}=\frac{\sin x \cos y+\cos x \sin y}{\cos x \cos y-\sin x \sin y} .
$$


On dividing both terms of the fraction on the right by $\cos x$ $\cos y$,

$$
\begin{aligned}
\tan (x+y) & =\frac{\frac{\sin x \cos y}{\cos x \cos y}+\frac{\cos x \sin y}{\cos x \cos y}}{\frac{\cos x \cos y}{\cos x \cos y}-\frac{\sin x \sin y}{\cos x \cos y}}=\frac{\frac{\sin x}{\cos x}+\frac{\sin y}{\cos y}}{1-\frac{\sin x \sin y}{\cos x \cos y}} \\
& =\frac{\tan x+\tan y}{1-\tan x \tan y} .
\end{aligned}
$$

If in the addition theorem for the tangent we change the sign of $y$ we obtain

since by 19.6

$$
\tan (x-y)=\frac{\tan x+\tan (-y)}{1-\tan x \tan (-y)}=\frac{\tan x-\tan y}{1+\tan x \tan y}
$$

24.5. Functions of Double an Angle. The addition and subtraction formulas listed in $\mathbf{2 4 . 1}$ form the starting point of a great many other formulas, the most important of which will be derived in this and the following sections of this lesson.

To begin with, let $y=x$; the addition formulas then read

$$
\begin{aligned}
& \sin (2 x)=2 \sin x \cos x, \\
& \cos (2 x)=\cos ^{2} x-\sin ^{2} x, \\
& \tan (2 x)=2 \tan x /\left(1-\tan ^{2} x\right) .
\end{aligned}
$$

In the second of these formulas we may put for $\cos ^{2} x$ its equal $1-\sin ^{2} x$ or for $\sin ^{2} x$ its equal $1-\cos ^{2} x$. The formula then assumes two other forms, namely,

$$
\cos (2 x)=1-2 \sin ^{2} x=2 \cos ^{2} x-1 .
$$

These formulas enable us to compute the functions of an angle if the functions of half that angle are known.

24.6. Functions of Half an Angle. If we solve the last equation for $\sin x$ and $\cos x$ respectively, we obtain

$$
\sin x= \pm \sqrt{\frac{1-\cos (2 x)}{2}}, \quad \cos x= \pm \sqrt{\frac{1+\cos (2 x)}{2}} .
$$


$2 x$ is any angle and $x$ is half that angle, hence we may write just as well

$$
\sin \frac{x}{2}= \pm \sqrt{\frac{1-\cos x}{2}}, \quad \cos \frac{x}{2}= \pm \sqrt{\frac{1+\cos x}{2}},
$$

and on dividing the first of these two expressions by the second we obtain a third formula, namely

$$
\tan \frac{x}{2}= \pm \sqrt{\frac{1-\cos x}{1+\cos x}} .
$$

When the functions of an angle are known, these formulas enable us to compute the functions of an angle half as large.

\subsection{Sums and Differences Expressed as Products.}

Since $\quad \sin (A+B)=\sin A \cos B+\cos A \sin B$, and $\sin (A-B)=\sin A \cos B-\cos A \sin B$,

we have by addition and subtraction

$$
\begin{aligned}
& \sin (A+B)+\sin (A-B)=2 \sin A \cos B, \\
& \sin (A+B)-\sin (A-B)=2 \cos A \sin B,
\end{aligned}
$$

and similarly

$$
\begin{aligned}
& \cos (A+B)+\cos (A-B)=2 \cos A \cos B, \\
& \cos (A+B)-\cos (A-B)=-2 \sin A \sin B .
\end{aligned}
$$

Let us put then

$$
\begin{aligned}
& A+B=x, A-B=y, \\
& A=\frac{1}{2}(x+y), B=\frac{1}{2}(x-y),
\end{aligned}
$$

and hence

$$
\begin{aligned}
& \sin x+\sin y=2 \sin \frac{1}{2}(x+y) \cos \frac{1}{2}(x-y), \\
& \sin x-\sin y=2 \cos \frac{1}{2}(x+y) \sin \frac{1}{2}(x-y), \\
& \cos x+\cos y=2 \cos \frac{1}{2}(x+y) \cos \frac{1}{2}(x-y), \\
& \cos x-\cos y=-2 \sin \frac{1}{2}(x+y) \sin \frac{1}{2}(x-y)
\end{aligned}
$$

These formulas enable us to express any sum or difference of two sines or cosines as a produet.

24.8. Illustrative Examples. Example 1. Given the functions of $45^{\circ}$ and $30^{\circ}$, to find the functions of $75^{\circ}$. 
Solution. $\quad \sin 45^{\circ}=\cos 45^{\circ}=\frac{1}{2} \sqrt{2}, \sin 30^{\circ}=\frac{1}{2}, \cos 30^{\circ}=\frac{1}{2} \sqrt{3}$, $\sin 75^{\circ}=\sin \left(45^{\circ}+30^{\circ}\right)=\sin 45^{\circ} \cos 30^{\circ}+\cos 45^{\circ} \sin 30^{\circ}$

$$
\begin{aligned}
& =\frac{1}{2} \sqrt{2} \cdot \frac{1}{2} \sqrt{3}+\frac{1}{2} \sqrt{2} \cdot \frac{1}{2}=\frac{1}{4}(\sqrt{6}+\sqrt{2}) \\
& =\frac{1}{4}(2.44949+1.41421)=0.96593,
\end{aligned}
$$

$\cos 75^{\circ}=\cos \left(45^{\circ}+30^{\circ}\right)=\cos 45^{\circ} \cos 30^{\circ}-\sin 45^{\circ} \sin 30^{\circ}$

$=\frac{1}{2} \sqrt{2} \cdot \frac{1}{2} \sqrt{3}-\frac{1}{2} \sqrt{2} \cdot \frac{1}{2}=\frac{1}{4}(\sqrt{6}-\sqrt{2})=0.25882$.

Example 2. Given the functions of $45^{\circ}$ and $30^{\circ}$, find the functions of $15^{\circ}$.

Solution. $\sin 15^{\circ}=\sin \left(45^{\circ}-30^{\circ}\right)=\sin 45^{\circ} \cos 30^{\circ}-\cos 45^{\circ} \sin 30^{\circ}$

$$
=\frac{1}{2} \sqrt{2} \cdot \frac{1}{2} \sqrt{3}-\frac{1}{2} \sqrt{2} \cdot \frac{1}{2}=\frac{1}{4}(\sqrt{6}-\sqrt{2})=0.25882,
$$

$\cos 15^{\circ}=\cos \left(45^{\circ}-30^{\circ}\right)=\cos 45^{\circ} \cos 30^{\circ}+\sin 45^{\circ} \sin 30^{\circ}$

$$
=\frac{1}{2} \sqrt{2} \cdot \frac{1}{2} \sqrt{3}+\frac{1}{2} \sqrt{2} \cdot \frac{1}{2}=\frac{1}{4}(\sqrt{6}+\sqrt{2})=0.96593 \text {. }
$$

Example 3. Given the functions of $45^{\circ}$, find the functions of $90^{\circ}$.

Solution. Using the formulas for double an angle, we have

$$
\begin{aligned}
& \sin 90^{\circ}=\sin \left(2 \times 45^{\circ}\right)=2 \sin 45^{\circ} \cos 45^{\circ}=2 \cdot \frac{1}{2} \sqrt{2} \cdot \frac{1}{2} \sqrt{2}=1, \\
& \cos 90^{\circ}=\cos \left(2 \times 45^{\circ}\right)=\cos ^{2} 45^{\circ}-\sin ^{2} 45^{\circ}=\left(\frac{1}{2} \sqrt{2}\right)^{2}-\left(\frac{1}{2} \sqrt{2}\right)^{2}=0, \\
& \tan 90^{\circ}=\tan \left(2 \times 45^{\circ}\right)=2 \tan 45^{\circ} /\left(1-\tan ^{2} 45^{\circ}\right)=2 /(1-1)=\infty .
\end{aligned}
$$

Example 3. Given the functions of $45^{\circ}$, find the functions of $22 \frac{1}{2}^{\circ}$.

Solution. From the formulas of half an angle, we have

$\sin 22 \frac{1}{2}^{\circ}=\sin \frac{45^{\circ}}{2}=\sqrt{\frac{1-\cos 45^{\circ}}{2}}=\sqrt{\frac{1-\frac{1}{2} \sqrt{2}}{2}}=\frac{1}{2} \sqrt{2-\sqrt{2}}=0.38268$, $\cos 22 \frac{1}{2}^{\circ}=\cos \frac{45^{\circ}}{2}=\sqrt{\frac{1+\cos 45^{\circ}}{2}}=\sqrt{\frac{1+\frac{1}{2} \sqrt{2}}{2}}=\frac{1}{2} \sqrt{2+\sqrt{2}}=0.92388$, $\tan 22 \frac{1}{2}^{\circ}=\tan \frac{45^{\circ}}{2}=\sqrt{\frac{1-\cos 45^{\circ}}{1+\cos 45^{\circ}}}=\sqrt{\frac{1-\frac{1}{2} \sqrt{2}}{1+\frac{1}{2} \sqrt{2}}}=\sqrt{2}-1=0.41421$.

Example 4. Transform $\sin 32^{\circ}-\sin 30^{\circ}$ into a product.

Solution. Applying the second formula of 24.7, we have $\sin 32^{\circ}-\sin 30^{\circ}=2 \cos \frac{32^{\circ}+30^{\circ}}{2} \sin \frac{32^{\circ}-30^{\circ}}{2}=2 \cos 31^{\circ} \sin 1^{\circ}$.

\section{EXERCISE 24}

1. Show that $\tan \left(45^{\circ}+x\right)=\frac{1+\tan x}{1-\tan x}$, and

$$
\tan \left(45^{\circ}-x\right)=\frac{1-\tan x}{1+\tan x}
$$


2. Given $\sin x=\frac{4}{5}$, $\sin y=\frac{15}{17}$, find $\cos x$ and $\cos y$, and then $\sin (x+y), \sin (x-y), \cos (x+y)$, and $\cos (x-y)$.

\section{Show that} Ans. $\frac{77}{85},-\frac{13}{85},-\frac{3}{8} \frac{6}{5}, \frac{84}{85}$.

$$
\begin{gathered}
\sin (3 x)=3 \sin x-4 \sin ^{3} x, \quad \cos (3 x)=4 \cos ^{3} x-3 \cos x, \\
\tan (3 x)=\left(3 \tan x-\tan ^{3} x\right) /\left(1-3 \tan ^{2} x\right) .
\end{gathered}
$$

4. If $t$ stands for $\tan x$, show that

$$
\sin (2 x)=\frac{2 t}{1+t^{2}}, \quad \cos (2 x)=\frac{1-t^{2}}{1+t^{2}}, \quad \tan (2 x)=\frac{2 t}{1-t^{2}} \cdot
$$

5. Show that $\cot (x+y)=(\cot x \cot y-1) /(\cot x+\cot y)$.

6. Prove that $\tan (x+y+z)=\frac{\tan x+\tan y+\tan z-\tan x \tan y \tan z}{1-\tan x \tan y-\tan y \tan z-\tan z \tan x}$. Prove the following identities :

7.

8.

$$
\begin{aligned}
& \frac{\sin A+\sin B}{\sin A-\sin B}=\frac{\tan \frac{1}{2}(A+B)}{\tan \frac{1}{2}(A-B),} \\
& \frac{\cos A+\cos B}{\cos A-\cos B}=-\frac{\cot \frac{1}{2}(A-B)}{\tan \frac{1}{2}(A+B)} . \\
& \frac{\sin A-\sin B}{\cos A+\cos B}=\tan \frac{1}{2}(A-B), \\
& \frac{\sin A-\sin B}{\cos A-\cos B}=-\cot \frac{1}{2}(A+B) .
\end{aligned}
$$

9. A flagpole 85 feet high stands on a building 40 feet high. At what distance from the foot of the building will the flagpole and the building subtend equal angles?

Ans. $66 \frac{2}{3} \mathrm{ft}$.

10. The area $T$ of a right triangle was determined from one side $b$, the hypotenuse $c$, and the included angle $A$. Afterwards it was found that an error $x$ had been made in measuring the angle $A$. Show that the corrected area is given by the formula

$$
T^{\prime}=T \cot A \tan (A+x) \text {. }
$$

If $T=100, T^{\prime}=101$, and $A=45^{\circ}$, show that $\tan x=1 / 201$. 
LESSON XXV - INVERSE TRIGONOMETRIC FUNCTIONS AND TRIGONOMETRIC EQUATIONS

25.1. Inverse Trigonometric Functions. Given such an equation as $y=\sin x$ it is frequently desirable to express $x$ in terms of $y$. Evidently $x$ is an angle whose sine is $y$; this we express symbolically by writing $x=\sin ^{-1} y$ and this is variously read,

$x$ is the inverse sine of $y$;

$x$ is the anti-sine of $y$;

$x$ is the arc-sine $y$;

$x$ is the angle whose sine is $y$.

Similarly, if $y=\cos x, x=\cos ^{-1} y$; if $y=\tan x, x=\tan ^{-1} y$; and similarly for each of the other functions.

It should be observed that the ${ }^{-1}$, here is not an exponent, that is, $\sin ^{-1} y$ does not mean $\frac{1}{\sin y}$. If we wish to use ${ }^{-1}$ as an exponent in connection with a trigonometric function, we should write $(\sin x)^{-1}$.

With the above definition of $\sin ^{-1} y$ in mind it is easily seen that

$$
\begin{array}{ll}
\sin \left(\sin ^{-1} y\right)=y, & \sin ^{-1}(\sin \theta)=\theta, \\
\tan \left(\tan ^{-1} y\right)=y, & \tan ^{-1}(\tan \theta)=\theta, \text { etc. }
\end{array}
$$

This explains why $\sin ^{-1}$ is called the inverse sine; looked upon as operations, each of a pair of operations such as sin and $\sin ^{-1}$ undoes the other, just as subtraction undoes addition and vice versa, or as division undoes multiplication and vice versa. 
25.2. Ambiguity of the Inverse Functions. When an angle is given, its sine has but one value, which may be found from a table of natural functions, but when the sine of an angle is given the angle may have any number of different values, and the same statement may be made regarding each of the other functions. For example, when the angle is $30^{\circ}$ its sine is 0.5 , but if the sine of an angle is known to be 0.5 the angle may be $30^{\circ}$ or $150^{\circ}$ or either of these two angles increased or decreased by any number of times $360^{\circ}$; that is, if $y=\sin ^{-1}(0.5), y$ may have any one of the values

$$
\begin{gathered}
30^{\circ}, 390^{\circ}, 750^{\circ} \text {, etc., } 150^{\circ}, 510^{\circ}, 870^{\circ} \text {, etc., } \\
-330^{\circ},-690^{\circ},-1050^{\circ} \text {, etc., }-210^{\circ},-570^{\circ},-930^{\circ} \text {, etc., }
\end{gathered}
$$

$\sin x$ is said to be a single-valued function of $x, \sin ^{-1} x$ a multiple-valued function of $x$. Similarly all the other inverse trigonometric functions are multiple-valued functions.

\subsection{Principal Values and General Values of Inverse} Functions. Of the many values which an inverse function may have, the numerically smallest value is called the principal value of the angle. If there are two_values which are numerically equal but have opposite signs, the positive value is considered the principal value of the angle. In the above example $30^{\circ}$ is the principal value of $\sin ^{-1}(0.5)$.

All the other values of the angle may then be expressed in terms of this principal value by means of a single formula

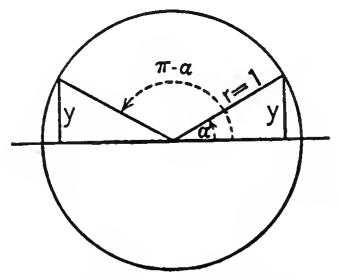

FIG. 67. $\theta=\sin ^{-1} y$. which is called the general value of the angle.

(a) Inverse sine. Let $\theta=\sin ^{-1} y$ and $\alpha$ be the principal value of $\theta$, then $\pi-\alpha$ is another value of $\theta$ (fig. 67). The remaining values then are

$2 n \pi+\alpha$ and $2 n \pi+(\pi-\alpha)$, 
where $n$ is any positive or negative integer and $\pi=180^{\circ}$. All these values may be combined into the single formula

$$
\sin ^{-1} y=n \pi+(-1)^{n} a \text {. }
$$

(b) Inverse cosine. If $\theta=\cos ^{-1} x$ and $\alpha$ the principal value of $\theta$, then $2 \pi-\alpha$ is another value of $\theta$ (fig. 68). The remaining values of $\theta$ are

$$
2 n \pi+\alpha \text { and } 2 n \pi+(2 \pi-\alpha) .
$$

All these values of $\theta$ are given by the formula

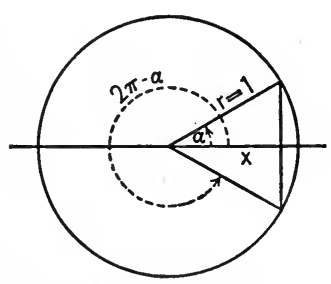

Fig. 68. $-\theta=\cos ^{-1} x$.

$$
\cos ^{-1} x=2 n \pi \pm a .
$$

(c) Inverse tangent. If $\theta=\tan ^{-1} y$ and $\alpha$ the principal value of $\theta$, then another value of $\theta$ is $\pi+\alpha$ (fig. 69). The

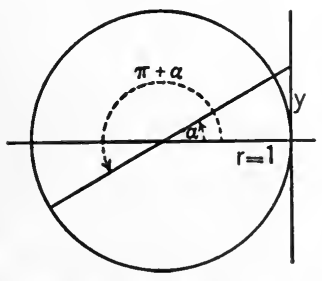

Fig. 69. $\theta=\tan ^{-1} y$. remaining values of $\theta$ are $n \pi+\alpha$ and $n \pi+(\pi+\alpha)$. All these values may be written

$$
\tan ^{-1} y=n \pi+a .
$$

Example 1. Let $\theta=\tan ^{-1} 1$. Then $\alpha=45^{\circ}$ or $\pi / 4$, and the general value of $\theta$ is $\theta=\tan ^{-1} 1$ $=n \pi+\pi / 4$, where $n$ is any positive or negative integer. By putting $n$ equal to $1,2,3$, etc., and $-1,-2,-3$, etc., we can write down as many of the different values of $\theta$ as we please.

Example 2. Let $\theta=\cos ^{-1}\left(\frac{1}{2} \sqrt{ } 3\right)$. Then $\alpha=30^{\circ}$ or $\pi / 6$, and the general value of the angle is $\theta=\cos ^{-1}\left(\frac{1}{2} \sqrt{ } 3\right)=2 n \pi \pm \pi / 6$, from which we can write down as many of the different values of $\theta$ as we please.

Example 3. Let $\theta=\sin ^{-1}\left(\frac{1}{2} \sqrt{ } 3\right)$. Then $\alpha=30^{\circ}$ or $\pi / 6$, and the general value of the angle is $\theta=\sin ^{-1}\left(\frac{1}{2} \sqrt{ } 3\right)=n \pi+(-1)^{n} \pi / 6$.

When $n=1, \theta=\pi-\pi / 6=5 \pi / 6=150^{\circ}$;

When $n=2, \theta=2 \pi+\pi / 6=13 \pi / 6=390^{\circ}$;

When $n=3, \theta=3 \pi-\pi / 6=17 \pi / 6=510^{\circ}$; etc.

25.4. Trigonometric Equations. An equation, in which the unknown quantity appears in the form of one or more trigono. 
metric functions, is called a trigonometric equation. Since an angle determined by means of one of its functions has an endless number of possible values, trigonometric equations have, as a rule, an endless number of solutions. The most obvious method of solving a trigonometric equation is to express all the functions of the unknown quantity in terms of some single one, as the sine or the cosine, and then to solve this equation considering this one function as the unknown quantity. We shall illustrate this method in the first example which follows. Frequently the work may be very much shortened by certain devices, some of which will be explained in the second and third examples.

Example 1. $2 \sin ^{2} x=3 \cos x$, to find $x$.

Solution. Since

$$
\sin ^{2} x=1-\cos ^{2} x \text {, we may write }
$$

$$
2\left(1-\cos ^{2} x\right)=3 \cos x
$$

or

$$
2\left(1-y^{2}\right)=3 y, \text { where } y=\cos x .
$$

Solving the equation $2\left(1-y^{2}\right)=3 y$, for $y$ we find

$$
y=\cos x=\frac{1}{2}, \text { or } y=\cos x=-2 \text {. }
$$

From the first of these values of $y$ we find the principal value of $x=\pi / 3$ or $60^{\circ}$, and the general value $x=2 n \pi \pm \pi / 3$.

The second value of $y$ makes $x$ imaginary since the cosine of an angle can not be less than -1 .

Example 2. If $\cos (2 \theta)=\sin (3 \theta)$, find $\theta$.

Solution. Since

$$
\begin{aligned}
& \sin (3 \theta)=\cos \left(\frac{\pi}{2}-3 \theta\right), \text { we may write } \\
& \cos (2 \theta)=\cos \left(\frac{\pi}{2}-3 \theta\right),
\end{aligned}
$$

from which

$$
2 \theta=2 n \pi \pm\left(\frac{\pi}{2}-3 \theta\right)
$$

or, solving for $\theta$,

$$
\theta=\frac{4 n+1}{10} \pi, \text { for the plus sign, }
$$

and

$$
\theta=\frac{1-4 n}{2} \pi, \text { for the minus sign. }
$$

We might equally well have put

$$
\cos (2 \theta)=\sin \left(\frac{\pi}{2}-2 \theta\right),
$$


and solved the equation

$$
\sin \left(\frac{\pi}{2}-2 \theta\right)=\sin (3 \theta),
$$

which would have given us

or, solving for $\theta$,

$$
\begin{aligned}
\frac{\pi}{2}-2 \theta & =n \pi+(-1)^{n}(3 \theta), \\
\theta & =\frac{2 n-1}{-6(-1)^{n}-4} \pi .
\end{aligned}
$$

This answer appears quite different from that obtained before, but the difference is only apparent. On substituting $n=0,1,2,-1,-2,-3$, etc., the two sets of values of $\theta$ are the same though their order is different. In either case we get

$$
\theta=\cdots \frac{-11 \pi}{10}, \frac{-7 \pi}{10}, \frac{-3 \pi}{10}, \frac{+\pi}{10}, \frac{5 \pi}{10}, \frac{9 \pi}{10}, \frac{13 \pi}{10}, \frac{17 \pi}{10}, \cdots
$$

Example 3. If $\sin (3 y)+\sin (2 y)+\sin y=0$, find $y$.

\section{Solution. By 24.7}

$$
\sin (3 y)+\sin y=2 \sin (2 y) \cos y,
$$

so that we may write

$$
2 \sin (2 y) \cos y+\sin (2 y)=0,
$$

from which we have

$$
\sin (2 y)=0, \quad \cos y=-\frac{1}{2} \text {. }
$$

From

$$
\sin (2 y)=0, \quad 2 y=n \pi+(-1) 0, \quad y=n \pi / 2,
$$

and from

$$
\cos y=-\frac{1}{2}, y=2 n \pi \pm(-\pi / 6)=2 n \pi \mp \pi / 6 \text {. }
$$

Example 4. $3 \cos x-4 \sin x=1$, find $x$.

Solution. Divide the equation by $\sqrt{3^{2}+4^{4}}=5$, then

$$
\frac{3}{5} \cos x-\frac{4}{5} \sin x=\frac{1}{5} \text {. }
$$

Now since $\left(\frac{3}{5}\right)^{2}+\left(\frac{4}{5}\right)^{2}=1$, we can find an angle $\alpha$ such that $\cos \alpha=\frac{3}{b}$, and $\sin \alpha=\frac{4}{5}$, and we may now write

But by 24.3

$$
\cos x \cos \alpha-\sin x \sin \alpha=\frac{1}{5} \text {. }
$$

hence

$$
\cos x \cos \alpha-\sin x \sin \alpha=\cos (x+\alpha),
$$

$$
\cos (x+\alpha)=\frac{1}{5} \text {. }
$$

By consulting a table of natural functions we find that the principal value of the angle

$$
x+\alpha=78^{\circ} 27.2^{\prime},
$$

and since

$$
\cos \alpha=\frac{3}{5}, \quad \alpha=53^{\circ} 7.3^{\prime},
$$

so that finally

$$
x+53^{\circ} 7.3^{\prime}=2 n \pi \pm 78^{\circ} 27.2^{\prime},
$$

and

$$
x=2 n \pi+25^{\circ} 19.9^{\prime} \text {, or } 2 n \pi-131^{\circ} 34.5^{\prime} \text {. }
$$




\subsection{Equations Involving Inverse Functions.}

Example 1. Prove that $\tan ^{-1}\left(\frac{5}{6}\right)-\tan ^{-1}\left(\frac{1}{11}\right)=\tan ^{-1} 1$.

Solution. Let us put $\tan ^{-1}\left(\frac{5}{6}\right)=A, \tan ^{-1}\left(\frac{1}{11}\right)=B$, then

$$
\tan A=\frac{5}{6}, \tan B=\frac{1}{11} \text {, }
$$

and the given expression becomes $A+B=\tan ^{-1} 1$, that is, we must show that $\tan (A+B)=1$.

Now by $\mathbf{2 4 . 4}$

$$
\tan (A+B)=\frac{\tan A+\tan B}{1-\tan A \tan B}=\frac{\frac{5}{6}+\frac{1}{11}}{1-\frac{5}{6} \times \frac{1}{11}}=\frac{\frac{61}{66}}{\frac{61}{66}}=1 .
$$

Example 2. Solve the equation $\cos ^{-1}(2 x)+\cos ^{-1}(3 x)=\cos ^{-1}\left(\frac{2}{3}\right)$.

Solution. Put $\cos ^{-1}(2 x)=A, \cos ^{-1}(3 x)=B$, then

$$
\begin{aligned}
& \cos A=2 x, \cos B=3 x, \\
& \sin A=\sqrt{1-4 x^{2}}, \sin B=\sqrt{1-9 x^{2}},
\end{aligned}
$$

and we may write

$$
A+B=\cos ^{-1}\left(\frac{2}{3}\right) \text {. }
$$

By taking the cosine of both the angle $A+B$ and the angle $\cos ^{-1}\left(\frac{2}{3}\right)$ we have $\cos (A+B)=\cos A \cos B-\sin A \sin B=\frac{2}{3}$, and expressing this in terms of $x$,

$$
2 x \cdot 3 x \pm \sqrt{1-4 x^{2}} \sqrt{1-9 x^{2}}=\frac{2}{3},
$$

the double sign being necessary since either radical may have etviner the plus or minus sign. Solving this equation for $x$ we obtain

$$
x= \pm \frac{1}{3} \text {. }
$$

On substituting these values in the original equation we find that the value $x=-\frac{1}{3}$ is inadmissible if we use only the principal values of the angles.

\section{EXERCISE 25}

1. Find the general values of the angles

$$
\sin ^{-1} 0, \cos ^{-1}\left(\frac{1}{2}\right), \tan ^{-1} 1, \cot ^{-1} \sqrt{ } 3, \sec ^{-1} 2 .
$$

Ans. $n \pi, 2 n \pi \pm \pi / 3, n \pi+\pi / 4, n \pi+\pi / 6,2 n \pi \pm \pi / 3$.

2. Show that

$$
\begin{aligned}
\sin ^{-1} x & =\cos ^{-1} \sqrt{1-x^{2}}=\tan ^{-1} \frac{x}{\sqrt{1-x^{2}}}=\cot ^{-1} \frac{\sqrt{1-x^{2}}}{x} \\
& =\sec ^{-1} \frac{1}{\sqrt{1-x^{2}}}=\csc ^{-1} \frac{1}{x}
\end{aligned}
$$

Considering principal values only, show that:

3. $\tan ^{-1} \frac{1}{2}+\tan ^{-1} \frac{1}{3}=\pi / 4$. 
4. $\tan ^{-1} m+\tan ^{-1}(1 / m)=\pi / 2$.

5. $\sin ^{-1} \frac{3}{5}+\cos ^{-1} \frac{1}{1} \frac{2}{3}=\tan ^{-1} \frac{56}{3}$.

Find both the principal value and the general value of the angle in the following equations :

6. $3 \cos ^{2} x=\sin ^{2} x$. Ans. Principal value $\pi / 3$, general value $2 n \pi \pm \pi / 3,2 n \pi \pm 2 \pi / 3$.

7. $\tan ^{2} x+4 \sin ^{2} x=3$. Ans. $\pi / 4, n \pi+\pi / 4$.

8. $\tan y+\cot y=\frac{10}{3}$. Ans. $\pi / 6, n \pi+\pi / 6, n \pi+\pi / 3$.

9. $\sin (10 x)=\sin (12 x)$. Ans. $0, n \pi /\left[10+(-1)^{n} 12\right]$.

10. $\sin (4 x)=\cos (5 x)$. Ans. $\pi / 18,\left(2 n_{\pi}-\pi / 2\right) /( \pm 5-4)$.

11. $\sin (3 x)+\sin (2 x)+\sin x=0$.

Find to the nearest minute, the angles less than $360^{\circ}$ which satisfy the following equations :

12. $\sin \theta+\cos \theta=0.5$. Ans. $114^{\circ} 18^{\prime}, 335^{\circ} 42^{\prime}$.

13. $\tan \theta+2 \cot \theta=10$.

Ans. $11^{\circ} 32^{\prime}, 84^{\circ} 10^{\prime}, 191^{\circ} 32^{\prime}, 264^{\circ} 10^{\prime}$.

14. If $\tan ^{-1}(1+x)+\tan ^{-1}(1-x)=\tan ^{-1}\left(\frac{2}{25}\right)$, show that $x= \pm 5$.

15. Find $x$ in

$$
\tan ^{-1}(2 x)+\tan ^{-1}(3 x)=3 \pi / 4 .
$$

Ans. 1 or $-\frac{1}{6}$. 


\section{LESSON XXVI-REVIEW}

1. Define a general angle. In what sense is this definition more general than the definition of an angle as given in elementary geometry? What is the complement of $95^{\circ}$ ? Of $-10^{\circ}$ ? When are two angles said to be supplementary?

2. Name three different systems of angle measurement. Compare the centesimal and the decimal system of angle measures. Draw a graph for converting grades into degrees and vice versa.

3. Define a radian. Give the exact and also the approximate value of a mil. Why is the radian called the natural unit? Draw a graph for converting radians into degrees and vice versa. Give the formula for the arc of an angle in terms of the radius and the angle. Give the formula for the area of a circular sector.

4. Define each of the trigonometric functions of a general angle. Name the three fundamental square relations. The three reciprocal relations. The two quotient relations. If the sine of an angle equals $\frac{5}{13}$, compute each of the other functions.

5. Prove the following equations:

$$
\begin{gathered}
\sin \left(360^{\circ}+\theta\right)=\sin \theta, \quad \sin \left(180^{\circ}+\theta\right)=-\sin \theta, \\
\sin \left(180^{\circ}-\theta\right)=\sin \theta, \quad \sin \left(90^{\circ}-\theta\right)=\cos \theta, \\
\sin \left(90^{\circ}+\theta\right)=\cos \theta, \sin \left(360^{\circ}-\theta\right)=\sin (-\theta)=-\sin \theta .
\end{gathered}
$$

Express as functions of $x$ each of the following : $\cos \left(180^{\circ}-x\right), \quad \tan \left(180^{\circ}-x\right), \quad \sin \left(x-90^{\circ}\right), \quad \cos \left(x-90^{\circ}\right)$. 
6. Follow the variations of the sine as the angle increases from $0^{\circ}$ to $360^{\circ}$.

Follow the variations of the cosine as the angle increases from $0^{\circ}$ to $360^{\circ}$.

Follow the variations of the tangent as the angle increases from $0^{\circ}$ to $360^{\circ}$.

7. Give from memory the values of the sine, cosine, and tangent of each of the angles, $0^{\circ}, 30^{\circ}, 45^{\circ}, 60^{\circ}, 90^{\circ}$. From these write down the values of the sine, cosine, and tangent of each of the angles $180^{\circ}, 150^{\circ}, 135^{\circ}, 120^{\circ}$.

8. Draw a unit circle and a central angle $\theta$, then draw lines representing each of the trigonometric functions.

9. Without using tables sketch each of the following curves :

$y=\sin x, y=\cos x, y=\tan x, y=\sec x, y=\csc x, y=\cot x$.

10. Sketch each of the curves :

$$
\begin{gathered}
y=3 \sin (2 x), y=\sin x+\cos x, y=5 \sin \left(\frac{1}{2} x+45^{\circ}\right), \\
y=2 \sin x-\frac{1}{2} .
\end{gathered}
$$

11. Define (a) uniform linear motion, $(b)$ uniform circular motion, (c) simple harmonic motion, (d) linear harmonic motion.

12. Define the terms (a) phase, (b) epoch, (c) displacement, (d) amplitude, used in connection with simple harmonic motion.

13. State Fourier's Theorem.

14. From a natural functions table find each of the following :

$\sin 27^{\circ}, \cos 56^{\circ}, \tan 113^{\circ}, \sin 145^{\circ} 20^{\prime}, \tan 165^{\circ} 45^{\prime} 30^{\prime \prime}, \cos 230^{\circ}$, $\sin 280^{\circ}, \tan 465^{\circ}, \sin -28^{\circ} 10^{\prime}, \cos -365^{\circ} 56^{\prime}, \tan 5678^{\circ}$.

15. State and prove the Law of Sines. State and prove the Law of Cosines. Give the formula for the area of any triangle 
in terms of two sides and the included angle. In terms of the three sides.

16. Enumerate the four cases of oblique triangles. Draw a figure showing that case II may have two distinct solutions. Explain how to solve the first case of triangles. The second case. The third case. The fourth case. Make up an example of your own illustrating each of the four cases.

17. Name the eight points of the compass from north to east and give the value of the angle determined by each point with the north and south line.

18. Prove the addition theorem for the sine and cosine.

19. State the subtraction theorems for the sine and cosine.

20. From the addition and subtraction theorems for the sine and cosine derive each of the following:

(a) The tangent of the sum of two angles.

(b) The sine and cosine of double an angle.

(c) The sine and cosine of $75^{\circ}$ without using tables.

21. From the formula for the cosine of double an angle derive the sine, the cosine, and the tangent of half an angle in terms of the functions of the whole angle.

22. Write from memory the formulas which express the sum or difference of two sines or cosines as a product of two functions.

23. Express as a product
(a) $\sin 40^{\circ}+\sin 20^{\circ}$
(b) $\cos 16^{\circ}-\cos 10^{\circ}$.

24. Find the values of $\sin ^{-1}\left(\frac{1}{2}\right), \sin \left(\cos ^{-1} \sqrt{ } \frac{3}{2}\right), \cos \left(2 \tan ^{-1} 3\right)$.

25. Show that for the principal values of the angles

$$
\tan ^{-1}\left(\frac{1}{2}\right)+\tan ^{-1}\left(\frac{1}{4}\right)+\tan ^{-1}\left(\frac{1}{13}\right)=\pi / 4 .
$$

26. Find the principal value of $x$ which satisfies the equation

$$
2 \cos (2 x)-\cos ^{2} x=0 \text {. }
$$

27. Find all the values of $x$ in $2 \sin x-3 \cos x=\sqrt{ } 5$. 


\section{CHAPTER IV}

\section{LOGARITHMS AND THEIR USE}

\section{LESSON XXVII - EXPONENTS AND LOGARITHMS}

\subsection{The Need of a Short Method of Computation.}

Every student is aware of the irksomeness of the ordinary methods of multiplication when the numbers to be multiplied contain five or more digits. The irksomeness is increased when such numbers are to be divided or if their square- or cube-root is to be extracted. Such operations as $\sqrt[5]{2}, \sqrt[7]{5}$, and $(7)^{3.45}$, are probably beyond his knowledge to perform. This shows that the common methods of computation are not only irksome but in many cases inadequate as well. To meet this difficulty in part, short methods of multiplication have been developed, some which have been extended to division, but most of these methods are unsatisfactory in that they can not be applied to the extraction of roots and the finding of powers of a number when the exponent is fractional. There is only one method which meets all the requirements in being simple, short, and applicable alike to multiplication, division, evolution, and involution. This is the method of exponents, or as it is more commonly called, the method of logarithms.

27.2. The Method of Exponents. It can be shown that every real number may be expressed as some power of any number (called the base) which is different from 1 and from 0. That is to say, given any number $a$, which is different from 
1 and from 0 , and any second number $N$, then a third number $n$ can always be found such that approximately $a^{n}=N$, and the approximation can be made as close as we please by carrying out $n$ to a sufficient number of decimals.

Suppose that $a$ is taken equal to 10 , and that for every value of $N$ the corresponding value of $n$ has been computed once for all. Suppose, furthermore, that all the numbers $N$ and their equal values $10^{n}$ are arranged in a table, let us say by writing the numbers $N$ in one line and their equal values $10^{n}$ in a second line thus,

$$
\begin{aligned}
N & =N_{1}, N_{2}, \quad N_{3}, \quad N_{4}, \cdots, \quad N_{p}, \cdots, \quad N_{q}, \ldots, N_{r}, \cdots, \\
10^{n} & =10^{n_{1}}, 10^{n_{2}}, 10^{n_{3}}, 10^{n_{4}}, \cdots, 10^{n_{p}}, \cdots, 10^{n_{q}}, \cdots, 10^{n_{r}}, \cdots .
\end{aligned}
$$

From this table whenever any number $N$ is given, $n$ is known also, and whenever any value $n$ is given the corresponding $N$ is known.

With such a table before us computation becomes an easy task. We shall consider each of the four operations mentioned in 27.1 in turn.

(a) To multiply any number $N_{p}$ by any other number $N_{q}$.

$$
N_{p} \cdot N_{q}=10^{n_{p}} \cdot 10^{n_{q}}=10^{n_{p}+n_{q}}=N_{r} .
$$

Hence to multiply two numbers $N_{p}$ and $N_{q}$ we add the corresponding $n$ 's and take from the table the number $N_{r}$ which corresponds to $n_{p}+n_{q}$.

(b) To divide any number $N_{p}$ by any other number $N_{q}$.

$$
N_{p} / \mathrm{V}_{q}=10^{n_{p}} / 10^{n_{q}}=10^{n_{p}-n_{q}}=N_{r} .
$$

Hence to divide a number $N_{p}$ by another number $N_{q}$ we subtract the $n_{q}$ of the second number from the $n_{p}$ of the first number, and from the table take the number $N_{r}$ which corresponds to $n_{p}-n_{q}$.

(c) To find the $k$ th power of any number $N$.

$$
N^{k}=\left(10^{n}\right)^{k}=10^{n k}=N_{r} \text {. }
$$


Hence to find the $k$ th power of any number $N$, multiply its $n$ by $k$ and from the table take the number $N_{r}$ which corresponds to $n k$.

(d) To find the $k$ th root of any number $N$.

$$
\sqrt[k]{ } N \doteq N^{1 / k}=\left(10^{n}\right)^{1 / k}=10^{n / k}=N_{r}
$$

Hence to find the $k$ th root of any number $N$, divide its $n$ by $k$, and from the table take the number $N_{r}$ which corresponds to $n / k$.

27.3. Definition of Logarithm. The numbers $n$ are called the common logarithms of the numbers $N$. 10 is called the base of the system of common logarithms. In general, if $a^{n}=N, n$ is called the logarithm of $N$ to the base $a$, and is written $n=\log _{a} N$. In words,

The logarithm of a number is the exponent of the power to which the base must be raised to produce that number.

Any number different from 1 and 0 might be chosen as a base to determine a system of logarithms, but in practice only two systems are used. One is the common system with the base 10 which is used exclusively in numerical computations. This system is also known as Briggs' system after Henry Briggs who, in 1617, published one of the first tables of common logarithms.

27.4. Fundamental Laws of Logarithms. Since logarithms are exponents, the fundamental laws of logarithms are essentially the same as the laws of exponents. We shall state in parallel columns the laws of exponents and the corresponding laws of logarithms.

LAws of Exponents LAws of Logaritums

$a^{m} \cdot a^{n}=a^{m+n}$.

(a) The logarithm of the product of two factors is equal to the sum of the logarithms of the factors, that is,

If $P=M \cdot N$, then $\log P=\log M+\log N$. 
LAws of Exponents. LAws of Logarithis.

$a^{m} / a^{n}=a^{m-n}$.

(b) The logarithm of the quotient of two num. bers is equal to the logarithm of the dividend diminished by the logarithm of the divisor, that is,

If $Q=M / N$, then $\log Q=\log M-\log N$.

$\left(a^{m}\right)^{n}=a^{m n}$

(c) The logarithm of any power of a number is equal to the logarithm of the number multiplied by the exponent of the power to which it is raised.

If $P=M^{n}$, then $\log P=n \cdot \log M$.

$\sqrt[n]{a^{m}}=a^{m / n} . \quad$ (d) The logarithm of any root of a number is equal to the logarithm of the number divided by the index of the root.

If $R=\sqrt[n]{ } M$, then $\log R=\frac{\log M}{n}$.

\subsection{Logarithms of Special Values.}

Since $a^{1}=a, \log _{a} a=1$, that is,

The logarithm of the base is 1 .

Again since, by 2.6, $a^{0}=1, \log _{a} 1=0$, that is,

The logarithm of 1 is 0 .

Also since, by $2.7, a^{-1}=1 / a, \log (1 / a)=-1$,

The logarithm of the reciprocal of the base is -1 .

By $27.4(b)$ we have

$$
\log (1 / N)=\log 1-\log N=0-\log N=-\log N \text {, or, }
$$

The logarithm of the reciprocal of any number is minus the logarithm of the number.

Definition. The logarithm of the reciprocal of a number is called the cologarithm of the number.

Now since $M / N=M \times(1 / N)$, we have $\log (M / N)=\log M+\log (1 / N)=\log M+\operatorname{colog} N$. 
The logarithm of a quotient of two numbers is equal to the logarithm of the dividend plus the cologarithm of the divisor.

27.6. Characteristic and Mantissa. Since in the common system of logarithms the base is 10 , we have furthermore $\log 1$ $=0, \log 10=1, \log \left(100=10^{2}\right)=2, \log \left(1000=10^{3}\right)=3$, etc. It follows that the common logarithm* of every number between 0 and 10 is some number between 0 and 1, that is, some proper fraction. Similarly the logarithm of every number between 10 and 100 is some number between 1 and 2, the logarithm of every number between 100 and 1000 is some number between 2 and 3, and so on. In general, therefore, the logarithm of a number consists of two parts, an integer and a fraction less than 1. The integral part is called the characteristic of the logarithm of the number, the fractional part is called the mantissa of the logarithm of the number. Thus if $541.7=$ $10^{2.7337}$, the logarithm of 541.7 is 2.73376 .2 is the characteristic and .73376 is the mantissa of the logarithm of 541.7. It follows from what has been said that if the characteristic of the logarithm of a number is 0 , the number lies between 1 and 10 and conversely. If the mantissa of the logarithm of a number is 0 , the number is some exact (integral) power of 10 , like $10,100,1000$, etc., provided the characteristic is positive, or some exact power of $\frac{1}{10}$, like $0.1,0.01,0.001$, etc., provided the characteristic is negative.

\subsection{Mantissa Independent of the Position of the Deci-} mal Point. Let us now take any number expressed as a power of 10 , as for example $541.7=10^{2.73376}$, and consider what effect it has on the logarithm if we move the decimal point of the number to the right or left. Let us first move the decimal point any number of places (let us say 3) to the right.

* Hereafter we shall omit the modifier " common," since all the logarithms dealt with in this and the following lessons are common logarithms. 
This is equivalent to multiplying the number by 1000 , that is,

$$
541,700=541.7 \times 1000=10^{2.73376} \times 10^{3}=10^{5.73376}
$$

so that $\log 541,700=5.73376$. The mantissa is the same as it was, but the characteristic has been increased by 3 , that is, by the number of places which the decimal point was moved to the right. It is obvious that if we had moved the decimal point any other number of places to the right, the effect on the logarithm would have been to increase its characteristic by an equal number, while the mantissa would still remain what it was, namely, .73376.

Let us next move the decimal point any number of places (say 5) to the left. This is equivalent to dividing the number 541.7 by 100,000 , so that,

$$
0.005417=541.7 / 100,000=10^{2.73376} / 10^{5}=10^{-3+.73376}
$$

from which $\log 0.005417=-3+.73376$. The mantissa is again the same as it was, but the characteristic is -3 , that is, $2-5$; in other words, the characteristic has been decreased by 5 , the number of places which the decimal point was moved to the left. We see therefore that

The mantissa of the logarithm of a number is independent of the position of the decimal point and dependent on the sequence of significant figures of the number.

The characteristic of the logarithm of a number is independent of the sequence of the significant figures of the number and dependent on the position of the decimal point.

27.8. Rule for the Characteristic. The mantissa of the logarithm of any number is readily found from a table of logarithms but the characteristic must be supplied by the student himself by some rule such as we shall now work out.

Suppose it is required to find the characteristic of the logarithm of 5417. We know that if the number were 5.417 
its characteristic would be 0 , for we have learned in 26.6 that the logarithm of every number between 1 and 10 has the characteristic 0. Now to obtain 5417 from 5.417 we must move the decimal point 3 places to the right, the effect of which would be to increase the characteristic by 3 ; hence the characteristic of the logarithm of 5417 must be 3 .

Again, let it be required to find the characteristic of the logarithm of 0.05417 . The characteristic of the logarithm of 5.417 is 0 ; to obtain 0.05417 from 5.417 we must move the decimal point 2 places to the left, the effect of which is to diminish the characteristic by 2 . The characteristic of the logarithm of 0.05417 is therefore -2 .

The foregoing reasoning leads to the following

Simple Rule for the Characteristic. Count the number of places which the decimal point of the given number must be moved to bring it immediately after the first significant figure. This gives the characteristic, which is positive if the decimal point had to be moved to the left, negative, if to the right.

Illustrations. The characteristic of the logarithm of 6789.45 is 3 , since the decimal point must be moved 3 places to the left in order to bring it into the position immediately after the first significant figure, which in this case is 6 .

The characteristic of the logarithm of 0.000084623 is -5 , since the decimal point must be moved 5 places to the right in order to bring it into the position immediately after the first significant figure, which in this case is 8 .

By reversing the foregoing rule we are enabled to determine the position of the decimal point of a number the characteristic of whose logarithm is known.

Illustration. The logarithm of a number is 2.73376 and the sequence of significant figures of the number as determined by a table of logarithms is 54170 ; to fix the position of the decimal point.

If the characteristic were 0 the decimal point would belong immediately after the figure 5 , but since the characteristic is 2 the decimal 
point must be moved 2 places to the right. The required number is therefore 541.70 .

If the characteristic had been -2 , we should have moved the decimal point in 2 places to the left, giving the number 0.05417 .

27.9. Negative Logarithms. A logarithm may be negative as well as positive, but before tables of logarithms may be consulted the logarithm must be so written that its fractional part is positive, for the mantissa, which is the only part of the logarithm that the table gives, is always positive. Suppose the logarithm of a number is known to be -1.56073 and we wish to find the number. If we first add and then subtract 2, we have for the logarithm of our number

$$
(-1.56073+2)-2=0.43927-2 \text { or }-2+0.43927 \text {, }
$$

that is to say the characteristic of our logarithm is -2 and its mantissa is .43927 . As a matter of fact instead of adding and subtracting 2 or some other number that will just make the fractional part of the logarithm positive, it is customary always to add and subtract 10 . Thus in the above example we should write

$$
\log N=-1.56073=(-1.56073+10)-10=8.43927-10,
$$

the characteristic now being $8-10$ or -2 , as before. Similarly if the logarithm of a number is $-3+.51188$, we should write

$$
\log N=(-3+.51188+10)-10=7.51188-10,
$$

the characteristic being $7-10$, or -3 .

\section{EXERCISE 27}

1. Write down the characteristics of the common logarithms of each of the following numbers :

$24.56,245.6,245600,24.56 \times 10^{7}, 2.456,0.2456,0.0002456$, $24.56 \times 10^{-10}$. 
2. How many digits are there in the integral part of the numbers whose logarithms are :

$2.45237,4.64109,0.34211,1.54782,6.87675,3.45322$ ?

3. The logarithm of 7453.6 is 3.87237 ; find the logarithms of each of the following numbers: 74.536, 0.74536, $0.00074536,74536,74536000,74.536 \times 10^{-7}$. Ans. 1.87237, $9.87237-10,6.87237$ - 10, etc.

4. To the number 625 corresponds the mantissa .79588 ; find the numbers whose logarithms are :

$1.79588,3.79588,0.79588,9.79588-10,7.79588-10,-1.79588$. Ans. 62.5, 6250, ..., 1/62.5.

5. Given $\log 62.5=1.79588$; find the logarithms of each of the following numbers:

$(62.5)^{2},(62.5)^{3}, \sqrt{ } 62.5, \sqrt[3]{ } 625,1 / 625,1 /(62.5)^{2},(6250)^{2} / \sqrt{6.25}$.

6. To the number 5 corresponds the mantissa .69897 ; find the logarithms of :

$$
5,25,0.2=\frac{1}{5}, 0.5,0.025,500,1.25, \sqrt[3]{5}, \sqrt{0.5}, 1 / \sqrt{50} .
$$

7. Given the logarithm of 5 as in problem 6 , find the number of digits in $5^{25}, 50^{10}, 250^{7}, 1.25^{100}$.

8. The amount $(S)$ of the principal $(P)$ put out at compound interest at the rate $i$ for $n$ years is given by the formula

$$
S=P(1+i)^{n} \text {. }
$$

Show that $\log S=\log P+n \log (1+i)$.

9. Find the cologarithms of each of the numbers in problem 6.

$$
\text { Ans. }-.69897=9.30103-10, \quad-1.39794=8.60205-10 \text {, }
$$
.69897, etc. 


\section{LESSON XXVIII - LOGARITHMIC COMPUTATION}

28.1. Use of a Table of Logarithms. Before we can go any further the student must learn how to find the mantissa of the logarithm of any number by the use of a table, and conversely, how to find from the same table the number which corresponds to any given mantissa. This is most easily accomplished with the personal assistance of the teacher or by reading the explanation in the introduction of the table (pp. viii, ix, x, Macmillan's Log. and Trig. Tables).

28.2. Logarithms are Exponents. The student should never forget that when he is dealing with logarithms he is dealing with exponents and that the method of logarithms differs only in form from the method of exponents. In the following examples we shall work each example first by the method of exponents (left) and then by the method of logarithms (right). The student should verify all the work and make sure that he understands each step.

Example 1. Find the product $P=247.23 \times 78.018 \times 0.00562 \times 1.4586$. With the aid of a table of logarithms we find

Method of Exponents

$$
\begin{aligned}
& 247.23= 10^{2.39310} \\
& 78.018= 10^{1.89220} \\
& .00562=10^{-3+.74974}= 10^{7.74974-10} \\
& 1.4586= 10^{0.16394} \\
& \hline P=10^{2.19898} \\
& P=158.12
\end{aligned}
$$

Method of Logarithms

$$
\begin{aligned}
\log 247.23 & =2.39310 \\
\log 78.018 & =1.89220 \\
\log .00562 & =7.74974-10 \\
\log 1.4586 & =0.16394 \\
\log P \quad & =2.19898 \\
P \quad & =158.12
\end{aligned}
$$

Example 2. Find $F=\frac{24.785 \times 7890.5}{632.1 \times 27.571}$. 
We may write $F$ in the form $24.785 \times 7890.5 \times \frac{1}{632.1} \times \frac{1}{27.571}$.

$$
\begin{aligned}
& \text { Method of Exponents } \\
& 24.785=\quad 10^{1.39419} \\
& 7890.5=\quad 10^{3.89710} \\
& \frac{1}{632.1}=10^{-2.80079}=10^{7.19921-10} \\
& \begin{aligned}
\frac{1}{27.571}=\frac{10^{-1.44046}}{}=10^{8.55954-10} \\
F=10^{1.05004} \\
F=11.221
\end{aligned}
\end{aligned}
$$

Method of Logarithm

$\log 24.785=1.39419$

$\log 7890.5=3.89710$

$\operatorname{colog} 632.1=7.19921-10$

$\operatorname{colog} 27.571=8.55954-10$

$\log F=1.05004$

$F=11.221$

The cologarithm of a number is found by subtracting the logarithm of the number from $10-10$ or 0 . Experienced computers do this without writing down the logarithm by subtracting (beginning on the left) each figure of the logarithm from 9 except the last figure, which is subtracted from 10.

Example 3. Find the value of $Q=\frac{(2.7183)^{5}}{\sqrt[3]{3.1416}}$.

We may write $Q=(2.7183)^{5} \times(3.1416)^{-\frac{1}{8}}$.

MeTHOD of Exponerts

$2.7183=10^{0.434298}$

$(2.7183)^{5}=10^{0.434298 \times 5}=10^{2.17149}$

$3.1416=10^{0.49715}$

$(3.1416)^{-\frac{1}{t}}=10^{0.49715 \times\left(-\frac{1}{3}\right)}$

$$
\begin{aligned}
& =10^{-0.16572}=10^{9.83428-10} \\
Q & =10^{2.00577} \\
Q & =101.34
\end{aligned}
$$

Method of LogarithM

$\log 2.7183=0.434298$

$5 \times \log 2.7183=2.17149$

$\operatorname{colog} 3.1416=9.50285-10$

$$
=29.50285-30
$$

$\frac{1}{8} \times \operatorname{colog} 3.1416=9.83428-10$

$$
\begin{aligned}
\log Q & =2.00577 \\
Q & =101.34
\end{aligned}
$$

In this example the student should notice that before we divided the cologarithm $9.50285-10$ by 3 we changed it so as to read $29.50285-30$. This was done so as to obtain a -10 in the quotient. Otherwise we would have obtained the quotient $3.16762-3 \frac{1}{3}$, which of course is the same as $9.83428-10$, but the first procedure is the easier. 
28.3. Exponential Equations. An equation in which the unknown quantity appears as an exponent is called an exponential equation. Such equations may frequently be solved with the aid of logarithms.

Example 1.

$$
3^{x}=15.348 \text {; find } x .
$$

Solution. Taking the common logarithm of both sides of the equation,

that is,

$$
x \log 3=\log 15.348,
$$

$$
\begin{aligned}
x(0.47712) & =1.18605 \\
x & =1.18605 / 0.47712 . \\
\log 1.18605 & =0.07410 \\
\operatorname{colog} 0.47712 & =0.32137 \\
\log x & =\overline{0.39547} \\
x & =2.4858 .
\end{aligned}
$$

Example 2. Solve the equation $e^{x}+e^{-x}=2.5$, where $e=2.7183$. Solution. Since $e^{-x}=\frac{1}{e^{x}}, e^{x}+\frac{1}{e^{x}}=2.5$,

$$
e^{2 x}-(2.5) e^{x}+1=0 .
$$

This is a quadratic equation in $e^{x}$, as is evident if we put $y$ for $e^{x}$, thus

from which

$$
y^{2}-(2.5) y+1=0 \text {, }
$$
or

$$
(y-2)\left(y-\frac{1}{2}\right)=0 \text {, }
$$

$$
y=e^{x}=2 \text {, and } y=e^{x}=\frac{1}{2} \text {. }
$$

Taking the common logarithms of both sides,

$$
\begin{aligned}
x \log e & =\log 2, \\
x(0.43429) & =0.30103, \\
x & =0.30103 / 0.43429=0.69315, \\
x \log e & =\log (0.5), \\
x(0.43429) & =9.69897-10=-0.30103, \\
x & =-0.30103 / 0.43429=-0.69315 .
\end{aligned}
$$

\section{EXERCISE 28}

By the use of logarithms find the results of the operations indicated below.

1. $87.43 \times 2.874 \times 0.6495$. Ans. 163.20 .

2. $1.457 / 783.5$. Ans. 0.0018595 .

3. $345.45 \times 0.00564 / 9.6754$. Ans. 0.20137 . 

4. $\frac{1.0056 \times 45.098 \times 456.67 \times 0.10109}{457.78 \times 67.875 \times 0.0098749}$.
Ans. 6.8234 .
5. $(3.031)^{3} \times(0.8653)^{4}$.
Ans. 15.612.
6. $\sqrt{34.687} \times \sqrt[3]{7.89403}$.
Ans. 11.727.
7. $(1.065)^{35.7}$.
Ans. 9.471.
8. $\frac{\sqrt[5]{32.488} \times(1.2017)^{3}}{\sqrt[3]{27.846} \times \sqrt{0.7634}}$
Ans. 1.3145 .

For the following problems use the formula in problem 8 , Exercise 27.

9. Find the amount of $\$ 100$ at compound interest for 10 years at $5 \%$.

Ans. \$162.89.

10. How long will it take a sum of money to double itself at $6 \%$ compound interest?

Ans. 11.89 yr.

11. At what rate of interest will $\$ 1$ grow to $\$ 1,000,000$ in $250 \mathrm{yr}$ ?

Ans. $5.68 \%$.

12. A father has two sons one of whom is twice as old as the other. At birth of each son he deposited $\$ 1000$ in a savings bank to accumulate at interest reckoned at $4 \%$ payable semiannually. Their combined accounts now total \$3579.10. How old are the sons? Ans. $9.5 \mathrm{yr}$., $19 \mathrm{yr}$.

13. Find $x$ in each of the following equations:

$$
\begin{gathered}
2^{x}=3, \quad 2^{x}=0.3, \quad(0.5)^{x}=0.15, \quad 5^{x}=4^{x+2} \quad 2^{x^{2}-2 x}=3^{x-1} \\
\text { Ans. } 1.585,-1.737,2.737,12.425,3.0685 \text { or } 0.5165 .
\end{gathered}
$$

14. Solve the equation $e^{x / 2}+e^{-x / 2}=4$, where $e=2.7183$.

Ans. $x= \pm 2.6339$. 
LESSON XXIX - APPLICATION OF LOGARITHMS TO THE MENSURATION OF PLANE FIGURES

29.1. Mensuration, which deals with the calculation of geometrical magnitudes such as distances, areas, and volumes, from data which make such calculation possible, furnishes abundant opportunity for the application of logarithms. In the exercise which follows we shall involve only such geometrical principles as will be familiar to most students who have studied plane geometry.

29.2. Example 1. A certain region on a map whose scale is 1 to 125,000 measures $24.75 \mathrm{sq}$. in. Find the number of acres in the area lepresented.

Solution. The area on the map is similar to the actual area represented, hence the area represented by the region on the map is

$$
A=24.75 \times(125,000)^{2} \text { sq. in. }
$$

Since there are $(12 \times 16.5)^{2} \mathrm{sq}$. in. in one square rod, and $160 \mathrm{sq}$. rd. in an acre

$$
\begin{array}{rl}
A=\frac{24.75 \times(125,000)^{2}}{(12 \times 16.5)^{2} \times 160} & \text { acres. } \\
& =1.39358 \\
\log 24.75 & =10.19382 \\
\log 125,000=5.09691 & =7.84164-10 \\
\log (125,000)^{2} & \\
\operatorname{colog} 12=8.92082-10 & \\
\operatorname{colog}(12)^{2} & =7.56504-10 \\
\operatorname{colog} 16.5=8.78252-10 & =7.79588-10 \\
\operatorname{colog}(16.5)^{2} & 4.78996 \\
\operatorname{colog} 160 & =61654 \text { acres. }
\end{array}
$$


Example 2. Find the radius of a circle whose area is equal to that of a triangle whose sides are $a=764.93, b=544.08, c=680.13$.

Solution. The area of a triangle in terms of the three sides is $\sqrt{s(s-a)(s-b)(s-c)}$ and the area of a circle in terms of its radius is $\pi r^{2}$, hence from the conditions of the problem we have

where

$$
\pi r^{2}=\sqrt{s(s-a)(s-b)(s-c)},
$$

from which

$$
\begin{aligned}
& s=(a+b+c) / 2, \\
& r=\frac{\sqrt[4]{s(s-a)(s-b)(s-c)}}{\sqrt{ } \pi} .
\end{aligned}
$$

$$
\begin{array}{rlrl}
2 s=a+b+c=764.93+544.08+680.13 & =1989.14 \\
s=994.57 & \log s \quad & =2.99764 \\
s-a=229.64 & \log (s-a) & =2.36105 \\
s-b & =450.49 & \log (s-b) & =2.65369 \\
s-c=314.44 & \log (s-c) & =2.49754 & \\
\log [s(s-a)(s-b)(s-c)] & =10.50992 & =2.62748 \\
\log \sqrt[4]{s(s-a)(s-b)(s-c)} & \\
\operatorname{colog} \pi=9.50285-10 & =9.75142-10 \\
\operatorname{colog} \sqrt{ } \pi & \log r=2.37890 \\
r & =239.28
\end{array}
$$

\section{EXERCISE 29}

1. Find the number of acres in a tract of land trapezoidal in shape whose parallel sides measure $786.9 \mathrm{ft}$. and $1035.5 \mathrm{ft}$., respectively, and the distance between these sides $346.8 \mathrm{ft}$.

Ans. 7.26.

2. The sides of a triangle are $65.6 \mathrm{ft}$., $78.9 \mathrm{ft}$., and $97.3 \mathrm{ft}$., respectively. Find the side of a square of equal area.

Ans. $50.74 \mathrm{ft}$.

3. How many square inches will the triangle in problem 2 occupy on a map drawn to the scale 1 to 1000 ?

Ans. .37 sq. in.

4. From the formula for the area of a triangle in terms of the sides show that the area of an equilateral triangle is $A=\frac{a^{2}}{4} \sqrt{ } 3$, where $a$ is the length of one side of the triangle. 
5. Find the radius of a circle whose area is equal to that of an equilateral triangle whose side measures $458.9 \mathrm{ft}$.

Ans. $170.4 \mathrm{ft}$.

6. How large would the radius of the circle in problem 5 have to be if the side of the triangle were twice as long? If the area of the triangle were twice as large? If the area of the triangle were one fourth as large?

7. Assuming that the distance of the earth from the sun is $92,500,000$ miles and that the earth describes a circle around the sun in $365 \frac{1}{4}$ days, find the number of miles the earth moves in one minute. Ans. 1105 miles.

8. Show that the area between two circles, one inside the other, is given by the formula

$$
A=\left(r_{1}+r_{2}\right)\left(r_{1}-r_{2}\right) \pi,
$$

where $r_{1}$ is the radius of the outer circle, $r_{2}$ the radius of the inner circle.

9. What will it cost to pave the area between two circles (problem 8) whose radii are $324.5 \mathrm{ft}$. and $165.7 \mathrm{ft}$., respectively, at $\$ 1.65$ per square yard ?

Ans. $\$ 44,835$.

10. A corner lot measures $205.4 \mathrm{ft}$. and $167.7 \mathrm{ft}$. from the corner along the two streets which intersect at any angle of $69^{\circ} 45^{\prime}$. What will be the amount of an assessment on this lot at the rate of $7.385 \&$ per square foot? Ans. $\$ 1193.30$. 
LESSON XXX-LOGARITHMIC AND EXPONENTIAL CURVES

30.1. The Exponential Curve, $y=a^{x}$.

(a) If a is greater than 1. (Fig. 70)

We have the following corresponding values of $x$ and $y$ :

$$
\begin{aligned}
& x=\quad 0, \quad 1,2,3,4 \text {, etc., } \\
& y=a^{0}=1, a^{1}=a, a^{2}, a^{3}, a^{4} \text {, etc., } \\
& x=-1, \quad-2, \quad-3, \quad-4 \text {, etc., } \\
& y=1 / a, 1 / a^{2}, 1 / a^{3}, 1 / a^{4} \text {, etc. }
\end{aligned}
$$

We observe

(1) When $x=0, y=1$, no matter what $a$ is, that is, every curve of the form $y=a^{x}$ passes through the point $(0,1)$.

(2) When $x$ is positive, $y$ also is positive, and increases with $x$ but much more rapidly. The curve, therefore, lies above the $x$-axis and diverges from it as $x$ increases ; the larger $a$ is the more rapidly the curve diverges from the $x$-axis.

(3) When $x$ is negative, $y$ is again positive, and decreases as the numerical value of $x$ increases; the larger $a$ is the more rapidly the curve converges to the $x$-axis.

(4) When $x$ is very large negative, $y$ is very small positive, and by taking $x$ sufficiently large negative, $y$ can be made less than any fixed positive number however small. Yet no matter how large negative $x$ is taken, $y$ can not be zero. In other words, if the curve $y=a^{x}$ is extended indefinitely to the left, it will approach the $x$-axis indefinitely near without ever meeting it.

Definition. A line which a curve approaches indefinitely near without ever meeting it is called an asymptote to the curve. It is 
limiting position of a tangent line to a curve as the point of contact moves to infinity. The $x$-axis is an asymptote to the curve $y=a^{x}$.

(b) If $a$ is less than 1 . Let us put $a=1 / b$, then if $a$ is less than $1, b$ will be greater than 1 , and the equation

$$
y=a^{x}=(1 / b)^{x}=b^{-x} \text {. }
$$

Let us now compare the two curves $y=b^{x}$ and $y=b^{x-}$. These two curves are symmetrically situated with respect to the $y$-axis; for every point on the first curve to the right of the $y$-axis there is a point on the second curve the same distance to the left of the $y$-axis, and conversely. Knowing the first

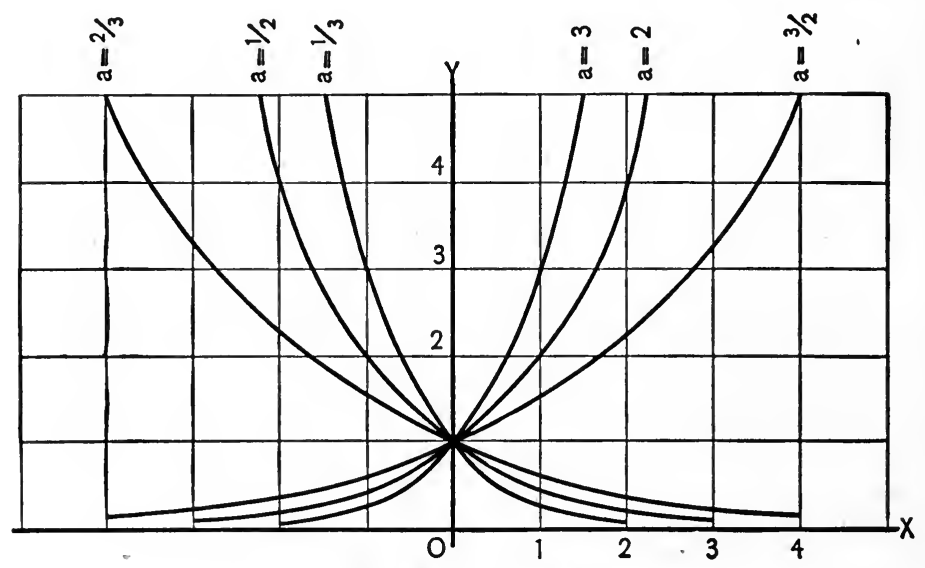

Fra. 70. $-y=a^{x}$.

curve, the second may therefore be obtained by simply reflecting the first on the $y$-axis. Figure 70 shows six curves of the type $y=a^{x}$.

\subsection{The Logarithmic Curve $y=\log _{a} x$.}

Let us first consider the case when $a=2$. We then obtain the following corresponding values of $x$ and $y$ : 
LOGARITHMIC AND EXPONENTIAL CURVES 197

$$
\begin{aligned}
& x=1, \quad 2, \quad 4, \quad 8, \quad 16 \text {, etc., } \\
& y=0, \quad 1, \quad 2, \quad 3, \quad 4 \text {, etc., } \\
& x=\frac{1}{2}, \quad \frac{1}{4}, \quad \frac{1}{8}, \frac{1}{16}, \frac{1}{32} \text {, etc., } \\
& y=-1,-2,-3,-4,-5 \text {, etc. }
\end{aligned}
$$

The resulting curve is shown in figure 71 . Similarly we may construct the logarithmic curve for any other base, but the process will be much simplified by means of the relation established in the next paragraph.

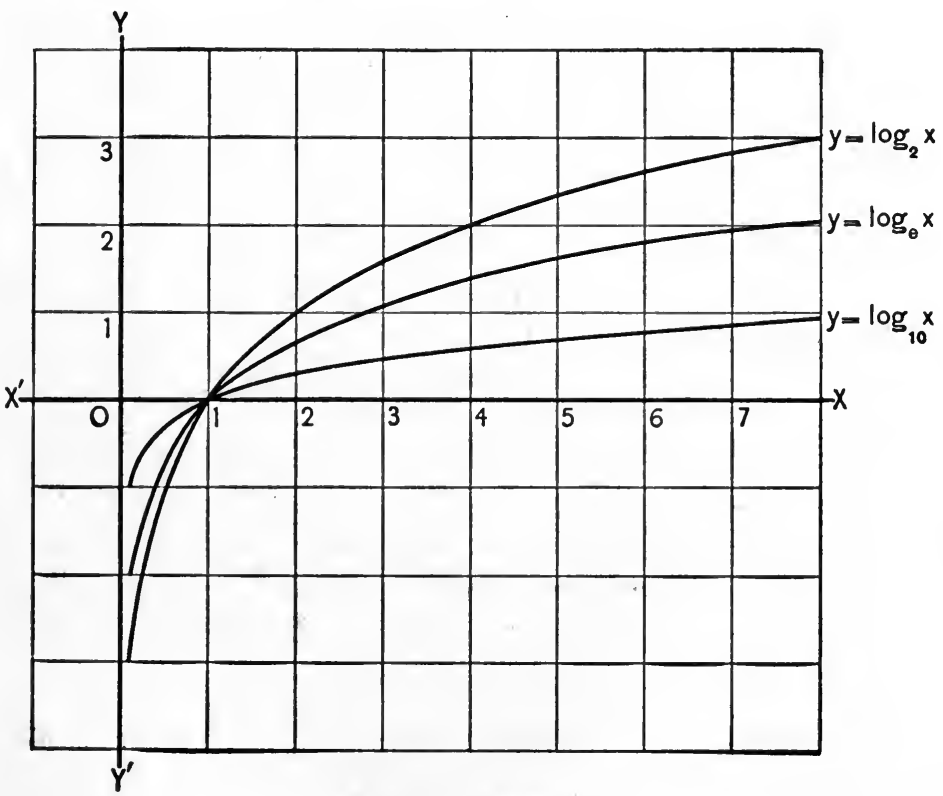

Fia. 71. $-y=\log _{a} x$.

30.3. Relation between $\log _{a} x$ and $\log _{b} x$. We will now show that the logarithms of numbers to two different bases are proportional, so that when the logarithms of numbers to 
one base are known we obtain the logarithms of the same numbers to any other base by multiplying the known logarithms by a certain ratio. It follows that having constructed the curve $y=\log _{2} x$ we may construct the curve $y=\log _{a} x$ by lengthening or shortening the ordinates of the given curve in a constant ratio.

Let

$$
\log _{a} x=y \text { and } \log _{b} x=y^{\prime},
$$

then by the definition of a logarithm

$$
a^{y}=x \quad \text { and } \quad b^{y^{\prime}}=x .
$$

Again, let us put

then

$$
\log _{a} b=c \text {, so that } b=a^{c} \text {, }
$$

from which

$$
a^{y}=x=a^{c y^{\prime}}
$$

that is,

$$
y=c y^{\prime} \text {, }
$$

$$
\log _{a} x=c \cdot \log _{b} x \text {, where } c=\log _{a} b \text {. }
$$

This shows that $\log _{a} x$ and $\log _{b} x$ are proportional, the common ratio being $\log _{a} b$.

If $x=a$ the preceding formula gives

$$
\log _{a} a=1=\log _{a} b \cdot \log _{b} a,
$$

which shows that

\section{$\log _{a} b$ and $\log _{b} a$ are reciprocals.}

It is now easy to construct the curve $y=\log _{a} x$ from the curve $y=\log _{2} x$. All we need to do is to multiply each ordinate of the latter curve by $c=\log _{a} 2$ and construct a new curve having these, values for ordinates. Thus to construct the curve $y=\log _{10} x$ we multiply the ordinates of the curve $y=\log _{2} x$ by $\log _{10} 2=0.30103$ (fig. 71).

30.4. Natural or Naperian Logarithms. Theoretically any positive number different from 1 may be used as the base of a system of logarithms but in practice only two numbers are so used. The first is the number 10, and the system of logarithms which this base determines is the common system used exclusively in making numerical computations.. The 
second number is the transcendental number $e$ which is defined as the limit of the expression $(1+1 / x)^{x}$ as $x$ approaches infinity. This limit may be shown to be equal to

$$
e=1+1+\frac{1}{2}+\frac{1}{2 \cdot 3}+\frac{1}{2 \cdot 3 \cdot 4}+\frac{1}{2 \cdot 3 \cdot 4 \cdot 5}+\text { etc. }
$$

from which its value is readily found to be

$$
e=2.71828 \cdots
$$

The system of logarithms to the base $e$ is called the Natural System or the Naperian System of logarithms. Natural logarithms are more easily computed than Common logarithms. The Common logarithms are then computed by means of the formula

$$
\log _{10} x=\log _{\bullet} x / \log _{\bullet} 10 .
$$

Notwithstanding the fact that natural logarithms were used in computing tables of common logarithms, common logarithmic tables are frequently the only tables accessible to the student. Occasionally natural logarithms are needed and when no table of natural logarithms is at hand they may be computed from the common logarithms by means of the formula

$$
\log _{\odot} x=\log _{10} x / \log _{10} e=2.30259 \times \log _{10} x .
$$

Common logarithms are universally used in numerical computations, natural logarithms in theoretical investigations.

\subsection{The Curve of Damped Vibrations,}

$$
y=a e^{-k x} \sin (b x+c) \text {. }
$$

Let

$$
\begin{aligned}
y_{1} & =\sin (b x+c), \quad(1) \quad y_{2}=a e^{-k x}, \\
y & =y_{1} y_{2}=a e^{-k x} \sin (b x+c),
\end{aligned}
$$

from which it appears that any ordinate of the required curve (3) is equal to the product of the corresponding ordinates of the two curves (1) and (2). We therefore proceed as follows: 
We first construct the separate curves (1) and (2) (fig. 72) and then construct the curve (3) by taking for its ordinates the products of corresponding ordinates of these two curves.

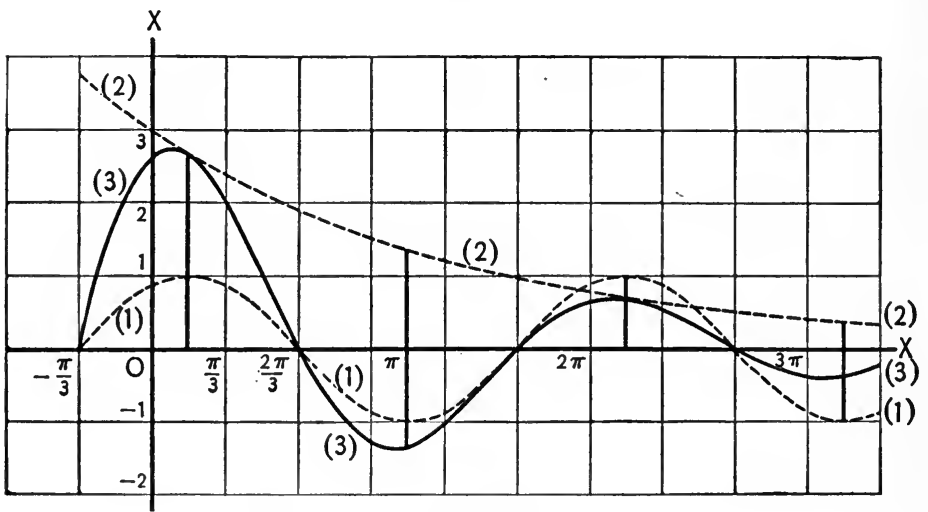

Fig. 72. $-y=a e^{-k x} \sin (b x+c)$.

The following observation should assist the student in carrying out the construction:

(a) $y_{1}$ is numerically less or at most equal to 1 , therefore the curve (3) lies entirely below the curve (2) touching it at the points of (1) whose ordinates are 1.

(b) If the curve (2) is repeated symmetrically below the $x$-axis, the required curve (3) will lie entirely above this curve, touching it at the points of (1) whose ordinates are -1 .

(c) Whenever $y_{1}$ equals $0, y$ also equals 0 , therefore the required curve (3) will cross the $x$-axis at the same points in which (1) crosses the $x$-axis.

(d) The ordinates of (2) are all positive, therefore the sign of the ordinates of (3) will depend on the sign of the ordinates of (1).

The shape of the required curve should now be apparent. It is a wave curve of constant wave-length but the amplitude 
of each wave is less than that of the preceding wave. The rate at which the amplitude decreases depends on the coefficient $k$. This coefficient is called the logarithmic decrement of the curve (3).

The curve of Damped Vibrations has a wide application in interpreting natural phenomena. Whenever a vibratory motion is retarded by friction or otherwise, the vibrations are damped vibrations. A pendulum vibrating in air or water, waves in a viscous fluid, and oscillatory discharges from an electric condenser are among the more common examples of damped vibration motion.

30.6. Logarithmic Trigonometric Functions. In the following lessons we shall constantly employ such functions as $\log \sin \theta, \log \tan \theta$, etc. These are the common logarithms of the natural functions and are called logarithmic trigonometric functions. To find these functions for any given value of $\theta$ we might first find the corresponding natural function of the angle and then find the logarithm of this natural function from a table of logarithms. This would require two tables, a table of natural functions and a table of logarithms. To avoid the trouble of using two tables a third table has been constructed, the logarithmic functions table, which gives at once the $\log \sin , \log \cos , \log \tan$, and $\log \cot$, for any given value of $\theta$.

As an illustration let us consider $\log \tan 35^{\circ} 46^{\prime}$.

$\tan 35^{\circ} 46^{\prime}=0.72034, \log \tan 35^{\circ} 46^{\prime}=\log 0.72034=9.85753-10$.

By the use of the logarithmic functions table we find directly log tan $35^{\circ} 46^{\prime}=9.85754$. This is slightly more accurate than the first result. In the logarithmic functions table the -10 is omitted, it being understood that when the characteristic is 9 or $8,-10$ is to be added in each case.

30.7. The Curve $y=\log \sin x$. As $x$ varies from $0^{\circ}$ to $90^{\circ}$ its sine varies from 0 to 1 , and the logarithm of the sine 
varies from $-\infty$ to 0 . As $x$ varies from $90^{\circ}$ to $180^{\circ}$ its sine varies from 1 to 0 and the logarithm of the sine varies from 0 to $-\infty$. In the third and fourth quadrants, the sine being negative, the logarithm of the sine is imaginary. In the fifth and sixth quadrants the values of $y$ are repetitions of the values in the first and second quadrants. The resulting curve (fig. 73) is a periodic curve with the period $2 \pi$.

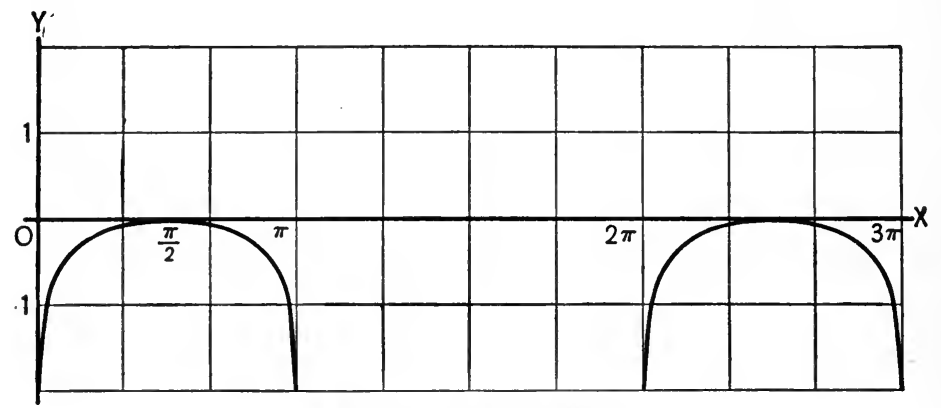

FIG. 73. $-y=\log \sin x$.

\section{EXERCISE 30}

1. Sketch on the same sheet of paper the three curves:

$$
y_{1}=3^{x}, y_{2}=3^{-x}, y=3^{x}+3^{-x} \text {. }
$$

2. Sketch on the same sheet of paper the four curves:

$$
y_{1}=e^{x}, y_{2}=e^{-x}, y=\left(e^{x}+e^{-x}\right) / 2, y=\left(e^{x}-e^{-x}\right) / 2 .
$$

3. The curve $y=\frac{a}{2}\left(e^{x / a}+e^{-x / a}\right)$ is called the common catenary. It is the curve formed by a chain or rope suspended between two points. Show that this curve is the magnification of the curve $y=\left(e^{x}+e^{-x}\right) / 2$ obtained by multiplying each abscissa and ordinate of this latter curve by $a$.

4. Prove that the curves $y=a^{x}$ and $x=\log _{a} y$ are the same curve, and that the curve $y=\log _{a} x$ is a like curve differently situated with respect to the coördinate axes. 
5. Sketch each of the curves $y=\log \cos x$ and $y=\log \tan x$.

6. Show that the two curves $y=\log \cos x$ and $y=\log \sec x$ are exactly alike, each being the reflection of the other on the $x$-axis.

7. Sketch each of the curves $y=e^{x} \cos x$, and $y=e^{-x} \cos x$.

8. Sketch the curve $y=1 /\left(\log _{2} x\right)$.

9. The work $W$ due to the expansion of a gas from one volume $V_{0}$ to another volume $V$, the temperature remaining constant, is given by $W=k \log _{e}\left(V / V_{0}\right)$, where $k$ is a constant depending on the initial volume and pressure of the gas. Plot a curve showing the relation between $V$ and $W$ for any volume up to $V=10$, when $V_{0}=1$, and $k=10^{7}$. 


\section{LESSON XXXI-LOGARITHMIC SOLUTION OF TRIANGLES. CASE I}

31.1. The Method of Logarithms. Logarithms are as important to modern science as steam power or electricity is to modern transportation. Laplace, the great French astronomer, said that the method of logarithms doubles the life of an astronomer by reducing to a few days the labors of many months, besides freeing him from the errors and disgust inseparable from long calculations. What is true of the astronomer is true in a large measure also of the navigator, the engineer, the surveyor, the statistician, the scientist, and all others who are called upon to perform long and intricate calculations. The method is universally applied to the solution of oblique and even of right triangles, for it is but seldom that the given parts of a triangle are expressible in such simple numbers as those made use of in lessons 22 and 23. Whenever the numbers contain three or more figures the logarithmic method saves time and makes for exactness. No experienced computer would think of using any other method when the numbers contain six and seven figures as they frequently do.

31.2. Checks in Computation. Whenever a problem involves lengthy numerical calculations the solution is not considered complete until it has been checked, by which is meant the application of some test which shows beyond a doubt that the answer is correct. This is necessary because "to err is human," that is to say, no one, not even the professional com- 
puter, can be sure that his work is free from errors in computation unless he has checked his work. To perform the computation a second time is no check, for the mind is so constituted that an error once made is likely to be repeated. The only sure checks are these two: either to have the computation performed independently by two different persons, or to arrive at the same result by two different methods. When a person works alone there is, of course, no alternative.

31.3. Schedules of Work. In logarithmic computations it is customary to first make out a schedule of work, by which is meant a systematic arrangement of the formulas to be employed and an orderly division of the space to be used in the computation. The schedule once completed, nothing should remain to be done except to fill in the numbers from the table and to perform the unavoidable additions.

This not only expedites the work but it also lessens the liability to error, since it enables the computer to give his whole attention for the time being to the mechanical part of work. A typical schedule of work will be given below.

31.4. General Solution of Case I. Given two angles and one side of a triangle, as $A, B, c$; to solve the triangle.

Solution. (a) To find the remaining angle subtract the sum of the two given angles from $180^{\circ}$.

(b) To find $a$. Use the law of sines, $a=\frac{c \sin A}{\sin C}$.

(c) To find $b$. Use the law of $\operatorname{sines,} b=\frac{c \sin B}{\sin C}$.

(d) Check. Use the law of cosines or the law of tangents (33.1).

SCHEDULE OF WORK

(a) $C=180^{\circ}-(A+B)=$
(b) $a=c \frac{\sin A}{\sin C}$
(c) $b=c \frac{\sin B}{\sin C}$. 
or

$$
\log c=
$$

$\log \sin A=$

$\operatorname{colog} \sin C=$

$\log a=$

$$
a=
$$

$\log c=$

$\log \sin B=$

$\operatorname{colog} \sin C=$

$\log b=$

$b=$

(d) Check.

$$
\begin{array}{cc}
c^{2}= & a^{2}+b^{2}-2 a b \cos C, \\
c-b=(c+b)= & a(a-2 b \cos C) . \\
\log (c-b)=\quad c+b= & a-2 b \cos C= \\
\log (c+b)= & \log a= \\
& \log (a-2 b \cos C)=
\end{array}
$$

All that remains to be done now is to fill in the numbers from the tables and perform the necessary additions. This will be illustrated by the following example.

\subsection{Numerical Example.}

$$
\begin{aligned}
\text { Grime } & \multicolumn{1}{c}{\text { ReQUIREd }} \\
A=65^{\circ} 46^{\prime} 16^{\prime \prime}, & a=337.65, \\
B=45^{\circ} 10^{\prime} 56^{\prime \prime}, & b=262.64, \\
{ }^{\circ} \mathrm{c}=345.78 ; & C=69^{\circ} 02^{\prime} 48^{\prime \prime} .
\end{aligned}
$$

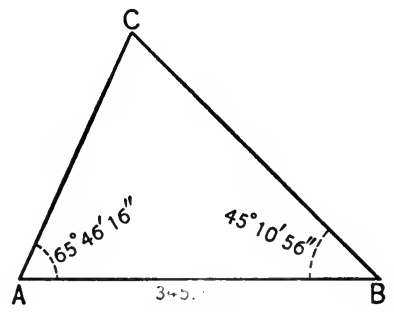

Fra. 74.

Solution.

(a) $C=180^{\circ}-(A+B)=180^{\circ}-\left(65^{\circ} 46^{\prime} 16^{\prime \prime}+45^{\circ} 10^{\prime} 56^{\prime \prime}\right)=69^{\circ} 02^{\prime} 48^{\prime \prime}$.
(b) $a=c \frac{\sin A}{\sin B}$.
(c) $b=c \frac{\sin B}{\sin C}$.

$\log 345.78=2.53880$

$\log 345.78=2.53880$

$\log \sin 65^{\circ} 46^{\prime} 16^{\prime \prime}=9.95996$

$\log \sin 45^{c} 10^{\prime} 56^{\prime \prime}=9.85086$

colog $\sin 69^{\circ} 02^{\prime} 58^{\prime \prime}=0.02970$ colog $\sin 69^{\circ} 02^{\prime} 58^{\prime \prime}=0.02970$

$\log a=\overline{2.52846}$

$\log b=\overline{2.41936}$

$a=337.65$

$b=\mathbf{2 6 2 . 6 4}$ 
(d) Check. $(c-b)(c+b)=a(a-2 b \cos C)$.

$$
\begin{array}{rr}
c-b=83.14, c+b=608.42, & a-2 b \cos C=149.81 . \\
\log (c-b)=1.91981 & \log a=2.52847 \\
\log (c+b)=\frac{2.78420}{4.70401} & \log (a-2 b \cos C)=\frac{2.17554}{4.70401}
\end{array}
$$

31.6. Chain of Triangles. Many practical problems involve not one only but a whole chain of triangles, each link of which must be solved in turn before the required distance or angle can be found. Sometimes the final result can be expressed by a single formula as in the following problem.

Problem. In figure 75 the side $A B$ and all the angles are known :
(1) $51^{\circ} 26^{\prime} 15^{\prime \prime}$,
(2) $38^{\circ} 27^{\prime} 25^{\prime \prime}$,
(7) $135^{\circ} 43^{\prime} 45^{\prime \prime}$,
(3) $90^{\circ} 06^{\prime} 20^{\prime \prime}$,
(8) $35^{\circ} 00^{\prime} 18^{\prime \prime}$,
(4) $25^{\circ} 50^{\prime} 00^{\prime \prime}$,
(9) $9^{\circ} 15^{\prime} 57^{\prime \prime}$,
(5) $113^{\circ} 35^{\prime} 30^{\prime \prime}$,
(10) $50^{\circ} 22^{\prime} 05^{\prime \prime}$,
(6) $40^{\circ} 34^{\prime} 30^{\prime \prime}$,
(11) $42^{\circ} 12^{\prime} 25^{\prime \prime}$,
(12) $87^{\circ} 25^{\prime} 30^{\prime \prime}$,

$A B=1000$; find $E F$.

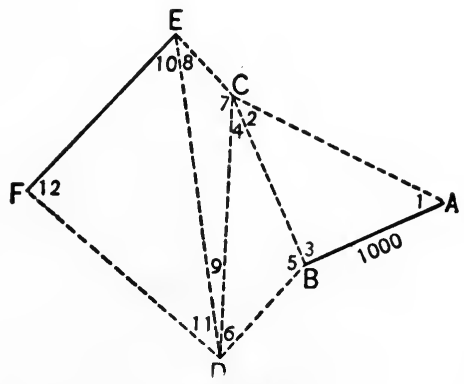

Fig. 75.

Solution. By the law of sines,

$$
\begin{aligned}
E F=\frac{\sin (11)}{\sin (12)} \cdot D E, \quad D E & =\frac{\sin (7)}{\sin (8)} \cdot C D, \quad C D=\frac{\sin (5)}{\sin (6)} \cdot B C, \\
B C & =\frac{\sin (1)}{\sin (2)} \cdot A B .
\end{aligned}
$$


Hence

$$
\begin{aligned}
& E F=\frac{\sin (11) \sin (7) \sin (5) \sin (1)}{\sin (12) \sin (8) \sin (6) \sin (2)} \cdot A B . \\
& \log \sin (11)=\log \sin 42^{\circ} 12^{\prime} 25^{\prime \prime}=9.82725 \\
& \log \sin (7)=\log \sin 135^{\circ} 43^{\prime} 45^{\prime \prime}=9.84388 \\
& \log \sin (5)=\log \sin 113^{\circ} 35^{\prime} 30^{\prime \prime}=9.96210 \\
& \log \sin (1)=\log \sin 51^{\circ} 26^{\prime} 15^{\prime \prime}=9.89316 \\
& \log A B=\log 1000 \quad=3.00000 \\
& \operatorname{colog} \sin (12)=\operatorname{colog} \sin 87^{\circ} 25^{\prime} 30^{\prime \prime}=0.00044 \\
& \operatorname{colog} \sin (8)=\operatorname{colog} \sin 35^{\circ} 00^{\prime} 18^{\prime \prime}=0.24136 \\
& \operatorname{colog} \sin (6)=\operatorname{colog} \sin 40^{\circ} 34^{\prime} 30^{\prime \prime}=0.18679 \\
& \operatorname{colog} \sin (2)=\operatorname{colog} \sin 38^{\circ} 27^{\prime} 25^{\prime \prime}=0.20626 \\
& \log E F=\overline{3.16124} \\
& E F=1449.6
\end{aligned}
$$

This problem furnishes a good illustration of the power of the method of logarithmic computation.

\section{EXERCISE 31}

1. Given $A=29^{\circ} 45^{\prime}, B=52^{\circ} 50^{\prime}, \quad c=37.9$; find the remaining parts of the triangle.

$$
\text { Ans. } a=19.0, b=30.5, C=97^{\circ} 25^{\prime} \text {. }
$$

2. Given $B=19^{\circ} 34.8^{\prime}, C=113^{\circ} 27.1^{\prime}, c=45.87$; solve the triangle.

3. Given $A=75^{\circ} 26^{\prime} 54^{\prime \prime}, C=35^{\circ} 54^{\prime} 35^{\prime \prime}, a=455.65$; find all the other parts of the triangle.

$$
\text { Ans. } B=68^{\circ} 38^{\prime} 31^{\prime \prime}, b=438.44, c=276.09 \text {. }
$$

4. Given $B=87^{\circ} 32^{\prime} 54^{\prime \prime}, C=64^{\circ} 17^{\prime} 06^{\prime \prime}, b=8.9765$; solve the triangle.

5. Find the distance $D F$ in figure 75 , not assuming $E F$ to be known.

6. In running a line $A B$ (fig. 76) an obstacle obstructed the line beyond $B$. To extend the line beyond the obstacle, a distance $B R=250 \mathrm{ft}$., making the angle $A B R=145^{\circ}$, was measured. At $R$ an angle $B R C=110^{\circ}$ was turned off. Find the 


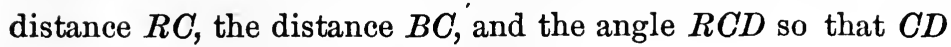
may be an extension of the line $A B$.

Ans. $R C=250 \mathrm{ft}$., $B C=409.6 \mathrm{ft}$.

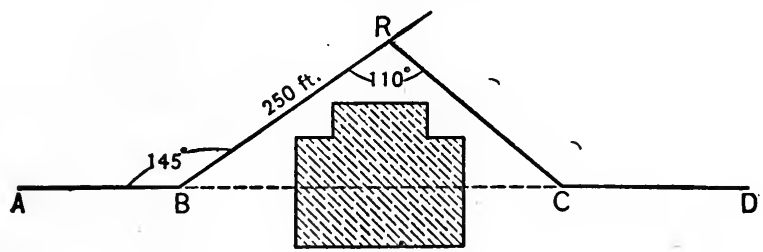

Fra. 76.

7. A cape as seen from board ship bears S. by W. The ship sails S.E. 50 miles and then the cape bears exactly west. How far is the cape from the second point of observation? Ans. 42.4 miles.

8. The commander of a field battery at $A$ wishes to determine the range of an enemy battery $B$ planted on the opposite side of a large river. He chooses a point $C$ from which $B$ is visible and measures the distance $A C$, also the angles $B A C$ and $B C A$. If $A C=500$ yards, $B A C=65^{\circ} 36^{\prime}$ and $B C A=110^{\circ} 54^{\prime}$, find the range of $B$ at $A$.

9. $P$ and $Q$ represent two forts 4.753 miles apart. An enemy warship is observed at $W$. The angle $P Q W=74^{\circ} 25^{\prime}$ and angle $Q P W=81^{\circ} 17^{\prime}$. Find the range of $W$ at each of the forts. 
LESSON XXXII - LOGARITHMIC SOLUTION OF TRIANGLES. CASE II

32.1. Two Solutions Possible. The case in which two sides of the triangle and an angle opposite one of these sides is given is known as Case II. This case is characterized by the fact that it may have two solutions, only one solution, or no solution at all, depending on the relative magnitudes of the given parts. When there are two solutions we are frequently left in doubt as to which of the two is the required solution; for this reason this case is known as the ambiguous, or doubtful, case of triangles. The difficulty here referred to is very much the same as that which we met in the solution of problems which required us to solve some quadratic equation; such problems it will be remembered may have two solutions, or only one solution, and in some cases no solution at all. In fact if we denote the given parts of the triangle by $a, b$, and $A$, we have by the law of cosines $a^{2}=b^{2}+c^{2}-2 b c \cos A$, and by solving this quadratic equation, considering $c$ as the unknown, we find for the third side of the triangle

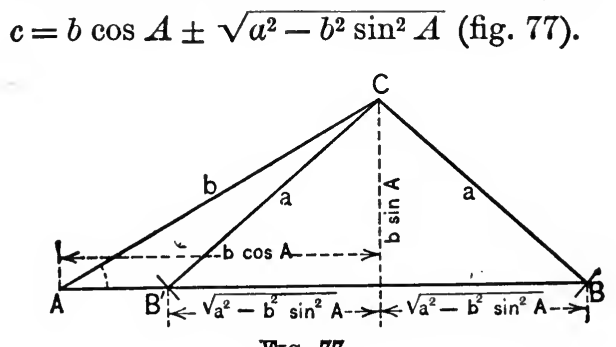

FTG. 77.

210 
This shows that for any given values of $a, b$, and $A, c$ has in general two values, but in order that $c$ may represent a side of some triangle, it must not only be real but must be positive as well. We might study the relations between $a$, $b$, and $A$, which will make $c$ positive from the above formula, but the beginner will find it easier to study the case geometrically.

32.2. Geometrical Discussion of the Doubtful Case. Construct an angle $B A C$ equal to the given angle $A$. Take $A C$ equal to the given side $b$ and from $C$ as a center, and a radius equal to the remaining given side $a$, draw a circle.

(a) The perpendicular distance from $C$ to $A B$ is $b \sin A$ (fig. 78). If $a$ is less than this perpendicular distance, the circle will not cut the side $A B$. In that case the triangle

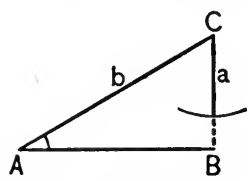

$a<b \sin A$

FIG. 78. has no solution. In other words the given parts do not form a triangle at all.

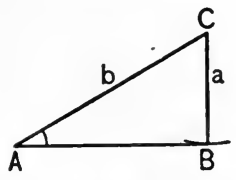

$a=b \sin A$

FIG. 79.

(b) If $a$ is just equal to the perpendicular distance from $C$ to $A B$, the circle will just touch the side $A B$ and the triangle has one solution (fig. 79). The resulting triangle is right angled at $B$.

(c) If $a$ is greater than the perpendicular distance from $C$ to $A B$, but less than $b$, the circle will cut the side $A B$ in two points $B$ and $B^{\prime}$; in that case two triangles are formed, namely, triangle $A B C$ and triangle $A B^{\prime} C$, each of which contains the given parts (fig. 80).

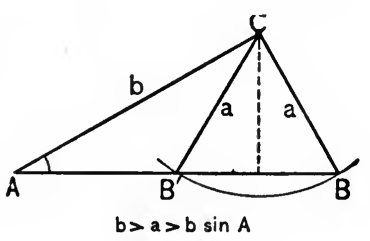

Fig. 80. 


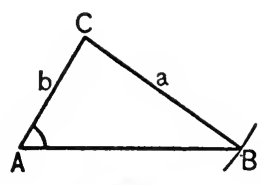

as b

FIG. 81 .

(d) If $a$ is greater than $b$, the circle will still cut the side $A B$ in two points $B$ and $B^{\prime}$ (fig. 81), but only one of the triangles thus formed will contain the given angle $A$, so that in this case there is only one solution.

(e) So far we have assumed the angle $A$ to be an acute angle. If $A$ is an obtuse angle (fig. 82), it is obvious that only one solution is possible, and that only in the case when $a$ is greater than $b$.

It appears from the foregoing discussion that two solutions can occur only when $a<b$. In every other case there is just one solution.

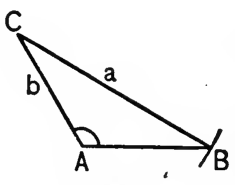

$A>90^{\circ}, a>b$

FIG. 82.

32.3. General Solution of Case II. Given two sides of a triangle and an augle opposite one of them, as $a, b$, and $A$; to solve the triangle.

Solution.

(a) To find $B$ and $B^{\prime}$. Use the law of sines, $\sin B=(b \sin A) / a$, then $B^{\prime}=180^{\circ}-B$.

(b) To find $C$ and $C^{\prime} . \quad C=180^{\circ}-(A+B), C^{\prime}=180^{\circ}-\left(A+B^{\prime}\right)$.

(c) To find $c$ and $c^{\prime}$. Use the law of sines, $c=(a \sin C) / \sin A$, $c^{\prime}=\left(a \sin C^{\prime}\right) / \sin A$.

(d) Check. Use the law of cosines or the law of tangents (33.1).

If $a>b$ there is just one solution, so that it would be useless to compute $B^{\prime}, C^{\prime}$, and $c^{\prime}$. If $a<b$ we are left in doubt whether there are two solutions, one solution, or no solution. This doubt can usually be removed by drawing to scale a rough sketch of the triangle. Or we may be governed by the following considerations :

If $\sin B$ comes out greater than 1 or, in case logarithms are used, if logs in $B$ comes out with a characteristic $0, B$ can 
not be found in the tables. In that case the triangle has no solution.

If $\sin B$ comes out equal to 1 or, in case logarithms are used, if $\log \sin B$ comes out exactly $0, B$ is a right angle and there is but one solution (fig. 79). In every other case $a<b$, there are two solutions.

32.4. Numerical Examples. Example 1. Given $a=72.31$, $b=125.3, A=35^{\circ} 15^{\prime}$. Do these parts form a triangle? If so, find the other parts.

Solution.

$$
\begin{aligned}
\log b & =2.09795 \\
\log \sin A & =9.76129 \\
\operatorname{col} l o g & =8.14080 \\
\log \sin B & =\overline{0.00004}
\end{aligned}
$$$$
\sin B=\frac{b \sin A}{a}
$$

This is an impossible value of $\log \sin B$, hence the given triangle has no solution, that is to say, the given parts do not form a triangle.

Example 2. Given $a=256.35, \quad b=456.35, \quad A=27^{\circ} 56^{\prime} 40^{\prime \prime}$; to find the other parts of the triangle.

Solution.

(a) To find $B$. To find $B^{\prime}$.

$$
\begin{array}{rlrl}
\log b & =2.65930 & \\
\log \sin A & =9.67082 & & \\
\sin B=\frac{b \sin A}{a} \quad & & B^{\prime}=180^{\circ}-B \\
\log \sin B & =\mathbf{9 . 9 2 1 2 8} & & 7.59116 \\
B & =56^{\circ} 32^{\prime} 07^{\prime \prime} & B^{\prime}=123^{\circ} 27^{\prime} 53^{\prime \prime}
\end{array}
$$

(b) To find $C$.

To find $C^{r}$. $C=180^{\circ}-(A+B)=95^{\circ} 31^{\prime} 13^{\prime \prime} \quad c^{\prime}=180^{\circ}-\left(A+B^{\prime}\right)=28^{\circ} 35^{\prime} 27^{\prime \prime}$

(c) To find $c$.

$$
\log a=2.40884
$$

$c=\frac{a \sin C}{\sin A} \quad \begin{aligned} \log \sin C & =9.99798 \\ \operatorname{colog} \sin A & =0.32918\end{aligned}$

$$
\log c=\overline{\mathbf{2 . 7 3 6 0 0}}
$$$$
c=544.50
$$

To find $c^{\prime}$.

$\log a=2.40884$

$$
\begin{aligned}
c^{\prime}=\frac{a \sin C^{\prime}}{\sin A} \quad \log \sin C^{\prime} & =9.67993 \\
\operatorname{colog} \sin A & =0.32918 \\
\log c^{\prime} & =\mathbf{2 . 4 1 7 9 5} \\
c^{\prime} & =\mathbf{2 6 1 . 7 9}
\end{aligned}
$$


(d) Check. $\quad(a-b)(a+b)=c(c-2 b \cos A)=c^{\prime}\left(c^{\prime}-2 b \cos A\right)$,

$$
\begin{array}{ll}
a-b=-200.00, & \log (a-b)=2.30103 n * \\
a+b=712.70, & \log (a+b)=\frac{2.85291}{5.15394 n}
\end{array}
$$

$$
\begin{aligned}
c-2 b \cos A & =-261.78, \\
\log c & =2.73600 n
\end{aligned}
$$

$\log (c-2 b \cos A)=\frac{2.41749}{5.15394 n}$

$$
c^{\prime}-2 b \cos A=-544.49 \text {. }
$$

$$
\log c^{\prime}=2.41795 n
$$

$$
\log \left(c^{\prime}-2 b \cos A\right)=\frac{2.73599}{5.15394 n}
$$

\section{EXERCISE 32}

1. Given $a=451, b=367, A=35^{\circ} 56^{\prime}$; find the remaining parts. Ans. $B=28^{\circ} 31.5^{\prime}, C=115^{\circ} 32.5^{\prime}, c=693.4$.

2. Given $b=293.6, c=357.9, B=52^{\circ} 27.7^{\prime}$; find the remaining parts.

3. Given $c=3454.6, a=5317.5, C=26^{\circ} 47^{\prime} 32^{\prime \prime}$; solve the triangle.

$$
\text { Ans. } \begin{aligned}
A & =43^{\circ} 56^{\prime} 00^{\prime \prime}, \quad B=109^{\circ} 16^{\prime} 28^{\prime \prime}, b=7234.5 ; \\
A^{\prime} & =136^{\circ} 04^{\prime} 00^{\prime \prime}, B^{\prime}=17^{\circ} 08^{\prime} 28^{\prime \prime}, b^{\prime}=2258.8 .
\end{aligned}
$$

4. Given $a=2.345, c=1.907, C=19^{\circ} 35^{\prime} 45^{\prime \prime}$; find the other parts.

5. A ferry crosses a river, which runs from west to east, from a point $A$ to a town $B$ which is in the direction N. $34^{\circ}$ $25^{\prime}$ E. at a distance of 5.97 miles from $A$. If the speed of the ferry in still water is 10 miles per hour and it takes 32 minutes for the ferry to cross, find the course of the ferry and the velocity of the current of the river. (Two answers, one of which is absurd.)

6. Two roads $a$ and $b$ meet at an angle of $35^{\circ}$ at a point $C$. From a point $B$ in $a$, which is at a distance of $7200 \mathrm{yd}$. from $C$, a cross-road $4500 \mathrm{yd}$. long is run to connect $a$ and $b$. At what distance from $C$ does this cross-road meet $b$ ? (Two answers.) Ans. 7686 yd. or 4110 yd.

* The $n$ indicates that the logarithm belongs to a negative number. 
7. An observer measures the angle subtended by a motor train consisting of 40 trucks at the moment that the last truck passes a point one mile due north of the observer. The angle measures $10^{\circ} 15^{\prime}$. Assuming each truck to be $15 \mathrm{ft}$. long and the distance between trucks to be equal to the length of a truck, compute the direction in which the train is moving and the distance of the observer from the head of the train. (Four answers.) 
LESSON XXXIII-LOGARITHMIC SOLUTION OF TRIANGLES. CASE III

33.1. The Law of Tangents. The third case of oblique triangles can be solved by using the law of cosines as was shown in 23.7, but this law is not adapted to the use of logarithms. For this reason we must first derive another law or formula known as

The Law of Tangents. In any triangle the sum of any two sides is to their difference as the tangent of one-half the sum of the opposite angles is to the tangent of one-half their difference. In symbols

$$
\frac{a+b}{a-b}=\frac{\tan \frac{1}{2}(A+B)}{\tan \frac{1}{2}(A-B)}
$$

33.2. Geometrical Proof of the Law of Tangents. Let $A B C$ (fig. 83) represent any triangle, $a, b, c$ the sides, and $A, B, C$ the corresponding opposite angles. With any vertex, as $C$, as a center and with a radius equal to $C A$, the

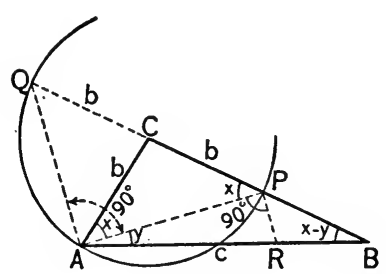

Fig. 83. shorter of two sides adjacent to $C$, draw a circle. Denote the points in which this circle euts $B C$ and $B C$ prolonged by $P$ and $Q$ respectively. Connect $A$ with $P$ and $Q$ and draw $P R$ parallel to $Q A$. Denote the angle $A P C$ by $x$ and the angle $P A B$ by $y$. Then it is easily seen that $A=x+y$ and $B=x-y$, hence on taking half the sum and half the difference we find

$$
x=\frac{1}{2}(A+B),
$$

$$
y=\frac{1}{2}(A-B) .
$$


From the similar triangles $B A Q$ and $B R P$ we have

$$
\frac{B Q}{B P}=\frac{A Q}{R P},
$$

but $B Q=B C+C A=a+b, \quad B P=B C-C A=a-b$, and from the right triangles $Q A P$ and $A P R$ we have

hence

$$
\begin{aligned}
& A Q=A P \tan x=A P \tan \frac{1}{2}(A+B), \\
& R P=A P \tan y=A P \tan \frac{1}{2}(A-B),
\end{aligned}
$$

$\frac{B Q}{B P}=\frac{A Q}{R P}$ becomes $\frac{a+b}{a-b}=\frac{A P \tan \frac{1}{2}(A+B)}{A P \tan \frac{1}{2}(A-B)}=\frac{\tan \frac{1}{2}(A+B)}{\tan \frac{1}{2}(A-B)}$,

which is the law of tangents.

33.3. Analytical Proof of the Law of Tangents. From the law of sines

$$
\frac{a}{b}=\frac{\sin A}{\sin B}
$$

Adding 1 to both sides of this equation,

$$
\frac{a}{b}+1=\frac{\sin A}{\sin B}+1, \text { or } \frac{a+b}{b}=\frac{\sin A+\sin B}{\sin B} \text {. }
$$

Subtracting 1 from both sides,

$$
\frac{a}{b}-1=\frac{\sin A}{\sin B}-1 \text {, or } \frac{a-b}{b}=\frac{\sin A-\sin B}{\sin B} .
$$

If we now divide (1) by (2) we obtain

$$
\begin{aligned}
\frac{a+b}{a-b}=\frac{\sin A+\sin B}{\sin A-\sin B} & =\frac{2 \sin \left(\frac{A+B}{2}\right) \cos \left(\frac{A-B}{2}\right)}{2 \cos \left(\frac{A+B}{2}\right) \sin \left(\frac{A-B}{2}\right)} \text { by 24.7. } \\
& =\frac{\tan \left(\frac{A+B}{2}\right)}{\tan \left(\frac{A-B}{2}\right)} .
\end{aligned}
$$


33.4. General Solution of Case III. Given two sides and the included angle of any triangle, as $a, b, C$; to solve the triangle.

Solution.

(a) To find $A$ and $B$, we first find $\frac{1}{2}(A+B)$ and $\frac{1}{2}(A-B)$.

To find $\frac{1}{2}(A+B)$ we have $A+B=180^{\circ}-C$, and

$$
\frac{1}{2}(A+B)=90^{\circ}-\frac{1}{2} C .
$$

To find $\frac{1}{2}(A-B)$ we have from the law of tangents,

$$
\tan \frac{1}{2}(A-B)=\frac{(a-b)}{(a+b)} \tan \frac{1}{2}(A+B) .
$$

Then $\quad A=\frac{1}{2}(A+B)+\frac{1}{2}(A-B), \quad B=\frac{1}{2}(A+B)-\frac{1}{2}(A-B)$.

(b) To find $c$. Use the law of sines, $\quad c=a \frac{\sin C}{\sin A}$.

(c) Check. Use the law of sines, $\quad c=b \frac{\sin C}{\sin B}$.

\subsection{Numerical Example.}

Given $\begin{aligned} a & =345.35, & \text { To find } A & =25^{\circ} 21^{\prime} 08^{\prime \prime}, \\ b & =267.78, & B & =19^{\circ} 23^{\prime} 26^{\prime \prime}, \\ c & =135^{\circ} 15^{\prime} 26^{\prime \prime} ; & c & =567.74 .\end{aligned}$

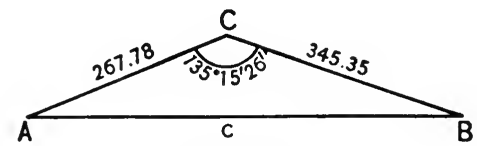

Fia. 84.

Solution. ( $(a)$ To find $A$ and $B$.

$$
\begin{aligned}
\frac{1}{2}(A+B) & =90^{\circ}-67^{\circ} 37^{\prime} 43^{\prime \prime} & =22^{\circ} 22^{\prime} 17^{\prime \prime} . \\
a-b & =77.57 . \quad a+b & =613.13 .
\end{aligned}
$$

$$
\begin{array}{r}
\log (a-b)=1.89969 \\
\operatorname{colog}(a+b)=7.21245
\end{array}
$$

$\tan \frac{1}{2}(A-B)=\frac{a-b}{a+b} \tan \frac{1}{2}(A+B), \quad \log \tan \frac{1}{2}(A+B)=\underline{9.61446}$

$A=22^{\circ} 22^{\prime} 17^{\prime \prime}+2^{\circ} 78^{\prime} 51^{\prime \prime}=25^{\circ} 21^{\prime} 08^{\prime \prime}$.

$B=22^{\circ} 22^{\prime} 17^{\prime \prime}-2^{\circ} 58^{\prime} 51^{\prime \prime}=19^{\circ} 23^{\prime} 26^{\prime \prime}$. 
(b) To find $c$.

$$
\begin{aligned}
\log a & =2.53826 \\
\log \sin C & =9.84752 \\
\operatorname{colog} \sin A & =0.36837 \\
\log c & =\overline{2.75414} \\
c & =\mathbf{s 6 7 . 7 1}
\end{aligned}
$$

(c) Check.

$$
c=b \frac{\sin C}{\sin B} \quad \begin{aligned}
\log \sin C & =\mathbf{9 . 8 4 7 5 2} \\
\operatorname{colog} \sin B & =\mathbf{0 . 4 7 8 8 5} \\
\log c & =\mathbf{2 . 7 5 4 1 5}
\end{aligned}
$$

\section{EXERCISE 33}

1 Given $a=0.895, b=0.671, C=15^{\circ} 38^{\prime}$; find the other parts. Ans. $A=128^{\circ} 22^{\prime}, B=36^{\circ} 00^{\prime}, c=0.308$.

2. Given $b=54.34, c=47.45, A=67^{\circ} 56.3^{\prime}$; solve the triangle.

3. Given $c=277.30, a=452.38, B=59^{\circ} 12^{\prime} 54^{\prime \prime}$; find the other parts.

$$
\text { Ans. } C=37^{\circ} 30^{\prime} 02^{\prime \prime}, A=83^{\circ} 17^{\prime} 04^{\prime \prime}, b=391.32 \text {. }
$$

4. Given $a=67,857, c=97,056, B=120^{\circ} 35^{\prime} 45^{\prime \prime}$; find the other parts.

5. A road is to be constructed between two points $A$ and $B$ on opposite sides of a dense forest $F$ (fig. 85). To determine the length of the road a line is run from $A$ to a point $P$ and from $P$ to $B$ and the following measures were taken: $A P=895 \mathrm{yd}$., $P B=782$ yd., angle $A P B=54^{\circ} 37^{\prime}$. Find the length of the road.

$$
\text { Ans. } 775.9 \text { yd. }
$$

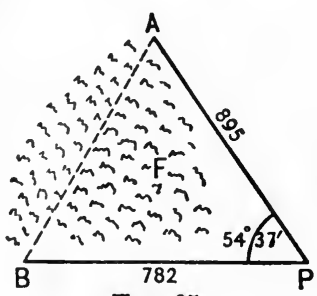

FTa. 85.

6. Two steamships, making 15 and 18.5 knots per hour respectively, met at sea. The course of the first ship was N.E. 
by N., the course of the second ship, N.N.W. How far apart were they $4 \mathrm{hr} .20 \mathrm{~min}$. after they met, and what was the direction of the first ship with respect to the second?

7. Two forces of $275.6 \mathrm{lb}$. and $765.9 \mathrm{lb}$., respectively, meet at an angle of $57^{\circ} 36.7^{\prime}$. Find the magnitude of the resulting force and its direction with respect to each of the component forces. Ans. $942.7 \mathrm{lb} ., 43^{\circ} 19^{\prime} .2,14^{\circ} 17^{\prime} .5$

8. An observer on board ship observes two warships at an angular distance of $42^{\circ} 32^{\prime}$. The interval between the flash and the report of a gun fired on board of each of the ships was noted to be 7 and 10 seconds, respectively. Find the distance between the two ships assuming the velocity of sound to be 1090 feet per second. (Suggestion. Use the law of cosines.) 
LESSON XXXIV - LOGARITHMIC SOLUTION OF TRIANGLES. CASE IV

34.1. The Tangent of Half the Angles. Case IV, like Case III, requires an additional formula before we can apply logarithms to its solution.

Let $A B C$ (fig. 86) represent any triangle, $A, B, C$ the angles, $a, b, c$ the corresponding opposite side, $s$ one-half the perimeter of the triangle, $O$ the center, and $r$ the radius of the inscribed circle, $O D, O E, O F$ the perpendiculars drawn from $O$ to the three sides of the triangle. We then know from ele-

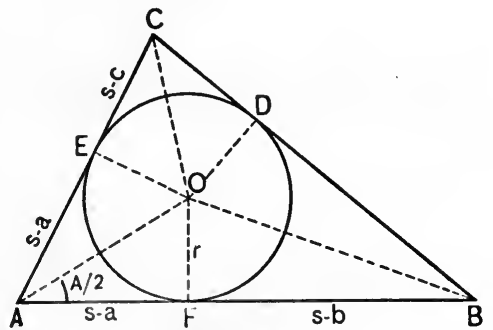

Fig. 86. mentary geometry that

$O A F=O A E=\frac{1}{2} A, O B F=O B D=\frac{1}{2} B, O C D=O C E=\frac{1}{2} C$,

$$
O D=O E=O F=r \text {, }
$$

and

$$
A F=A E, B D=B F, D C=C E \text {, }
$$

hence

$A F+B D+D C=A F+(B D+D C)=A F+a=\frac{1}{2}$ perimeter $=s$, so that $A F=s-a$, and similarly $B F=s-b, C D=s-c$. Finally from the right triangles $O F A, O F B$, and $O D C$ we have

that is,

$$
\begin{gathered}
\tan (O A F)=O F / A F, \quad \tan (O B F)=O F / B F, \\
\tan (O C D)=O D / C D,
\end{gathered}
$$

$$
\tan \frac{1}{2} A=\frac{r}{s-a}, \tan \frac{1}{2} B=\frac{r}{s-b}, \quad \tan \frac{1}{2} C=\frac{r}{s-c} .
$$


34.2. The Angles in Terms of the Sides. It remains only to express $r$ in terms of the sides of the triangle. This is quite easy, for

triangle $A B C=$ triangle $O B C+$ triangle $O C A+$ triangle $O A B$

$$
=\frac{1}{2} r a+\frac{1}{2} r b+\frac{1}{2} r c=\frac{1}{2}(a+b+c) r=s r,
$$

and from 23.8 we have the area of the same triangle

$$
=\sqrt{s(s-a)(s-b)(s-c)},
$$

so that

$$
s r=\sqrt{s(s-a)(s-b)(r-c)},
$$

from which

$$
\boldsymbol{r}=\frac{\sqrt{(\boldsymbol{s}-\boldsymbol{a})(\boldsymbol{s}-\boldsymbol{b})(\boldsymbol{s}-\boldsymbol{c})}}{\boldsymbol{s}}
$$

34.3. Analytical Proof of the Tangent Half-angle Formula. Since

$$
\begin{gathered}
2 s=a+b+c, 2(s-a)=-a+b+c, 2(s-b)=a-b+c, \\
2(s-c)=a+b-c .
\end{gathered}
$$

From the law of cosines (23.4)

therefore

$$
\cos A=\left(b^{2}+c^{2}-a^{2}\right) /(2 b c),
$$

$$
\begin{aligned}
1-\cos A & =1-\left(b^{2}+c^{2}-a^{2}\right) /(2 b c)=\frac{a^{2}-\left(b^{2}+c^{2}-2 b c\right)}{2 b c} \\
& =\frac{a^{2}-(b-c)^{2}}{2 b c}=\frac{(a+b-c)(a-b+c)}{2 b c}=\frac{4(s-c)(s-b)}{2 b c}
\end{aligned}
$$

$1+\cos A=1+\left(b^{2}+c^{2}-a^{2}\right) /(2 b c)=\frac{b^{2}+c^{2}+2}{2 b c} b c-a^{2}$

$$
=\frac{(b+c)^{2}-a^{2}}{2 b c}=\frac{(b+c+a)(b+c-a)}{2 b c}=\frac{4 s(s-a)}{2 b c} \text {. }
$$

Finally, by (24.6)

$$
\begin{aligned}
\tan \frac{A}{2} & =\sqrt{\frac{1-\cos A}{1+\cos A}}=\sqrt{\frac{(s-b)(s-c)}{s(s-a)}} \\
& =\frac{1}{s-a} \sqrt{s(s-a)(s-b)(s-c)}=r /(s-a) .
\end{aligned}
$$


34.4. General Solution of Case IV. Given the three sides, $a, b, c$, of any triangle; to find the three angles.

Solution.

(a) First compute the values of $s, s-a, s-b$, and $s-c$, as follows : Add the three sides and divide the sum by 2 to find $s$, then subtract in turn $a, b$, and $c$. Check, $(s-a)+(s-b)+(s-c)=s$.

(b) Find $\log r$ from the formula in 34.1, thus $\log r=\frac{1}{2}[\log (s-a)+\log (s-b)+\log (s-c)+\operatorname{colog} s]$.

(c) Find $A, B$, and $C$, from the formulas in 13.1, thus

$$
\begin{aligned}
& \log \tan \left(\frac{1}{2} A\right)=\log r+\operatorname{colog}(s-a), \\
& \log \tan \left(\frac{1}{2} B\right)=\log r+\operatorname{colog}(s-b), \\
& \log \tan \left(\frac{1}{2} C\right)=\log r+\operatorname{colog}(s-c) .
\end{aligned}
$$

(d) Check. $A+B+C=180^{\circ}$.

34.5. Numerical Examples. Given $a=588.45, b=672.01$, $c=875.32$; find the angles.

Solution.

$$
s=(a+b+c) / 2, \quad r=\sqrt{(s-a)(a-b)(s-c) / s},
$$

$\tan \left(\frac{1}{2} A\right)=r /(s-a), \quad \tan \left(\frac{1}{2} B\right)=r /(s-b), \quad \tan \left(\frac{1}{2} C\right)=r /(s-c)$.

$$
\begin{array}{rlrl}
s & =1067.89 & \operatorname{colog} s & =6.97148 \\
s-a & =479.44 & \log (s-a) & =2.68074 \\
s-b & =395.88 & \log (s-b) & =2.59757 \\
s-c & =\frac{192.57}{1067.89} & \log (s-c) & =2.28459 \\
\text { Check. } & & \log r^{2}= & \mathbf{4 . 5 3 4 3 8} \\
\log r & =2.26719
\end{array}
$$

$$
\log r=2.26719
$$

$\operatorname{colog}(s-a)=7.31926$

$\operatorname{colog}(s-b)=7.40243$

$\operatorname{colog}(s-c)=7.71541$

$\log \tan \left(\frac{1}{2} A\right)=\overline{\mathbf{9 . 5 8 6 4 5}}$

$\log \tan \left(\frac{1}{2} B\right)=9.66962$

$\log \tan \left(\frac{1}{2} C\right)=9.98260$

$$
\begin{aligned}
& \frac{1}{2} A=21^{\circ} 06^{\prime} 01.6^{\prime \prime}, \quad A=42^{\circ} 12^{\prime} 03^{\prime \prime}, \\
& \frac{1}{2} B=25^{\circ} 02^{\prime} 52.7^{\prime \prime}, \quad B=50^{\circ} 05^{\prime} 45^{\prime \prime} \text {, } \\
& \frac{1}{2} C=43^{\circ} 51^{\prime} 09.6^{\prime \prime}, \quad C=87^{\circ} 42^{\prime} 19^{\prime \prime} \text {, }
\end{aligned}
$$

Noтe. The excess of $8^{\prime \prime}$ in the check is due to necessary inaccuracy of a five-place table. By using a six-place table the angle sum will be found to be $180^{\circ}$ to the nearest second. 


\section{EXERCISE 34}

1. Given $a=841, b=879, c=876$; find the angles of the triangle. Ans. $A=57^{\circ} 16^{\prime}, B=61^{\circ} 33^{\prime}, C=61^{\circ} 11^{\prime}$.

2. Given $a=43.32, b=65.76, c=75.09$; find the angles.

3. Given $a=0.56740, b=0.35626, c=0.82354$; find the angles.

Ans. $A=34^{\circ} 34^{\prime} 03^{\prime \prime}, B=20^{\circ} 52^{\prime} 12^{\prime \prime}, C=124^{\circ} 33^{\prime} 48^{\prime \prime}$.

4. Given $a=345.78, b=674.32, c=465.80$; find the angles.

5. $A, B$, and $C$ are three ports whose distances from each other are known. $A B=84.6$ miles, $B C=57.2$ miles, $C A=$ 64.2 miles. If $B$ is due west of $A$, and if $C$ is somewhere north of the line $A B$, find the direction of $C$ from each of the ports $A$ and $B$. Ans. N. $47^{\circ} 29^{\prime}$ W., N. $40^{\circ} 40^{\prime}$ E.

6. A surveyor wishes to determine the distance-between two points $A$ and $B$ (fig. 87) separated by a marsh, but he has no instrument with which to measure angles. He selects a

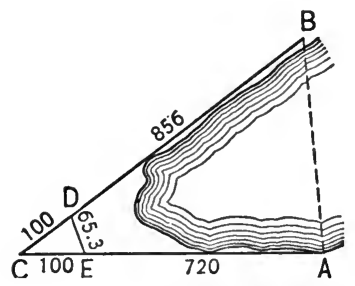

FIG. 87 . point $C$ from which both $A$ and $B$ are visible and measures the distances $A C=720$ yd., $B C=856$ yd. $\mathrm{He}$ then drives two stakes, $D$ and $E$, each $100 \mathrm{yd}$. from $C, D$ in a line with $A$, and $E$ in a line with $B$, and measures the distance between these two stakes. If $D E=65.3 \mathrm{yd}$., find the

distance $A B$. Ans. 530.4 yd.

7. In fig. 86 show that

$$
O A^{2}=O F^{2}+A F^{2}=r^{2}+(s-a)^{2}=b c(s-a) / s,
$$

and therefore,

$$
\sin \left(\frac{1}{2} A\right)=\sqrt{\frac{(s-b)(s-c)}{b c}}, \cos \left(\frac{1}{2} A\right)=\sqrt{\frac{s(s-a)}{b c}} .
$$




$$
\tan \left(\frac{1}{2} A\right)=\frac{\sin \left(\frac{1}{2} A\right)}{\cos \left(\frac{1}{2} A\right)}=\sqrt{\frac{(s-b)(s-c)}{s(s-a)} .}
$$

Write down similar formulas for the angles $\frac{1}{2} B$ and $\frac{1}{2} C$.

One of these formulas is frequently used in preference to the formulas in $\mathbf{3 4 . 1}$ and $\mathbf{3 4 . 2}$ when only one of the angles of a triangle is required to be known.

8. Three forces, of $365.6 \mathrm{lb}$., $427.8 \mathrm{lb}$, and $289.5 \mathrm{lb}$., respectively, are in equilibrium. At what angles do they meet? 


\section{LESSON XXXV - MISCELLANEOUS PROBLEMS INVOLVING TRIANGLES}

35.1. Other Cases of Oblique Triangles. There are many cases of oblique triangles besides the four fundamental or principal cases that have been discussed in detail in the four preceding lessons. It is possible to solve the triangle when there are given :

(a) The three perpendiculars from the vertices of the angles to the opposite sides.

(b) One side, the perpendicular from the opposite vertex, and either the sum or the difference of the other two sides.

(c) One angle, the perpendicular upon the opposite side, and either the sum or the difference of the other two sides.

(d) One angle, the side opposite, and the sum of the other two sides.

(e) The angles and the sum or difference of two sides.

$(f)$ One angle, the side opposite this angle, and the sum or difference of the squares of the other two sides.

(g) Two sides and the sum or difference of the opposite angles.

(h) The angles and the sum of the sides.

In fact the number of such cases is inexhaustible. The solution of each of these cases may, however, be made to depend directly or indirectly on one or more of the four fundamental cases or upon the formulas used in their solution. As an example we will consider the first case mentioned. 
Example. Given the three altitudes of a triangle to solve the triangle.

Solution. Denote the altitudes by $h_{a}, h_{b}$, and $h_{c}$ and put $a^{\prime}=1 / h_{a}$, $b^{\prime}=1 / h_{b}, c^{\prime}=1 / h_{c}$, then if $T$ represents twice the area of the triangle we have

$T=a \cdot h_{a}=b \cdot h_{b}=c \cdot h_{c}$,
and therefore $\quad a=T \cdot a^{\prime}, \quad b=T \cdot b^{\prime}, \quad c=T \cdot c^{\prime}$,

and

$s=(a+b+c) / 2=T\left(a^{\prime}+b^{\prime}+c^{\prime}\right) / 2=T \cdot s^{\prime}$, where $s^{\prime}=\left(a^{\prime}+b^{\prime}+c^{\prime}\right) / 2$, also $\quad s-a=T\left(s^{\prime}-a^{\prime}\right), s-b=T\left(s^{\prime}-b^{\prime}\right), s-c=T\left(s^{\prime}-c^{\prime}\right)$.

If we now substitute these values in the last formula of Exercise 31 (8), we have

$\tan \left(\frac{1}{2} A\right)=\sqrt{\frac{(s-b)(s-c)}{s(s-a)}}=\sqrt{\frac{\left(s^{\prime}-b^{\prime}\right)\left(s^{\prime}-c^{\prime}\right)}{s^{\prime}\left(s^{\prime}-a^{\prime}\right)},}$

and similarly

$\tan \left(\frac{1}{2} B\right)=\sqrt{\frac{\left(s^{\prime}-c^{\prime}\right)\left(s^{\prime}-a^{\prime}\right)}{s^{\prime}\left(s^{\prime}-b^{\prime}\right)}}, \tan \left(\frac{1}{2} C\right)=\sqrt{\frac{\left(s^{\prime}-a^{\prime}\right)\left(s^{\prime}-b^{\prime}\right)}{s^{\prime}\left(s^{\prime}-c^{\prime}\right)}}$.

This gives the angles in terms of the altitudes, from which the sides may be found by case IV.

35.2. System of Triangles. Many practical problems involve several triangles which must be solved in succession either in whole or in part before the required distance or angle can be found. One of the most famous of such problems is the following, sometimes referred to as

Hanson's Problem. To find the distance between two inaccessible points.

Analysis of the Problem. Let $P$ and $Q$ (fig. 88) represent the two inaccessible points. Select two points, $A$ and $B$, from which both $P$ and $Q$ are visible. Measure the distance $A B$, also the angles $A B Q, A B P, B A P, B A Q$. Then

(a) In the triangle $A B P$, we have

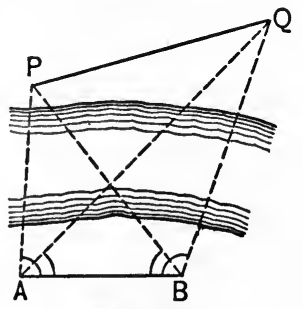

FIG. 88. 
given one side, $A B$, and the two adjacent angles, $A B P$ and $B A P$, hence we can compute $A P$ and $B P$ (Case I).

(b) In the triangle $A B Q$, we have given one side, $A B$, and the two adjacent angles, $A B Q$ and $B A Q$, hence we can compute $A Q$ and $B Q$ (Case I).

(c) In the triangle $A P Q$, we have given two sides, $A P$ and $A Q$, and the included angle $P A Q$ (=angle $B A P$-angle $B A Q$ ), hence we can compute the required distance $P Q$ (Case III).

(d) Check. In the triangle $B P Q$, we have given two sides, $B P$ and $B Q$, and the included angle $P B Q$ (=angle $A B Q$-angle $A B P$ ), hence we can compute the required distance $P Q$ a second time (Case III).

\subsection{Auxiliary Geometrical Constructions. Frequently} the intermediate triangles needed in the solution are not apparent from the figure but must be supplied by means of auxiliary geometrical constructions. A well-known instance of this kind is

The Three Point Problem. To find the distance of a point from each of three points whose distances from each other are known.

Analysis of the Problem. Let $A, B, C$ (fig. 89) represent the three points whose distances from each other, namely $B C=a$,

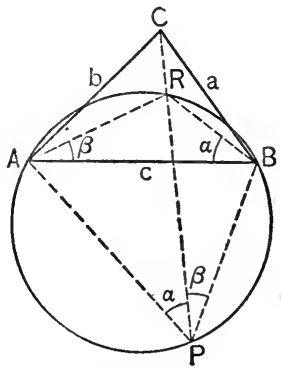

Fig. 89. $C A=b, A B=c$, are known. Let $P$ represent the point whose distances from $A, B$, and $C$ are to be found.

Measure the angles

$$
A P C=\alpha \text { and } C P B=\beta .
$$

Suppose a circle is drawn through the three points $P, A$, and $B$, and let $R$ represent the point in which this circle intersects the line $P C$. Join $R$ to $A$ and to 
$B$. Then angle $A B R=$ angle $A P R=\alpha$, being inscribed angles subtended by the same arc, and for the same reason angle $B A R=$ angle $R P B=\beta$.

(a) In the triangle $A B R$, the side $A B$ and the two angles $A B R$ and $B A R$ are known, hence we can compute the sides $A R$ and $B R$ (Case I).

(b) In the triangle $A B C$, the three sides are known, hence we can compute the angles $A, B$, and $C$ (Case IV).

(c) In the triangle $A R C$, the two sides, $A R$ and $A C$ and the included angle $R A C$ (=angle $A$-angle $\beta$ ) are known, hence we can compute the angle $A C R$ (Case III).

(d) In the triangle $A P C$, one side $A C$ and two angles, $A P C$ and $A C P$, are known, hence we can compute the remaining sides $P A$ and $P C$ (Case I).

(e) In the triangle $B P C$, one side $B C$ and two angles, $B P C$ and $B C P$ (=angle $C$-angle $A C R$ ) are known, hence we can compute the remaining sides $P B$ and $P C$ (Case I).

$(f)$ Check. Compare the value of $P C$ as found in $(d)$ with the value of $P C$ as found in $(e)$.

\section{EXERCISE 35}

1. $P$ is a point, invisible and inaccessible from $A$, whose distance from $A$ is to be ascertained. From $A$ a line $A B C$ was run, $B$ and $C$ being two points from each of which $P$ was visible. The following measures were taken :

$$
\begin{aligned}
A B & =257.9 \mathrm{yd} ., \\
\text { angle } A B P & =135^{\circ} 36.5^{\prime}, \\
B C & =210.6 \mathrm{yd} ., \\
\text { angle } B C P & =70^{\circ} 45.5^{\prime} .
\end{aligned}
$$

Find the distance $A P$.

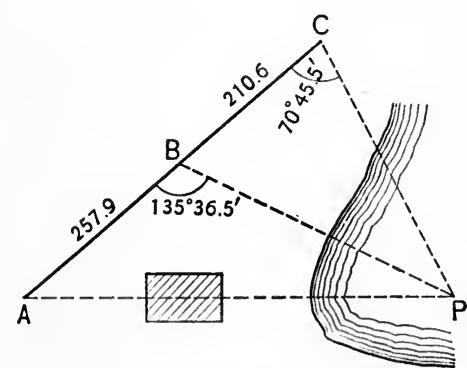

Frg. 90. 
2. To find the distance between two mountain peaks, $M$ and $M^{\prime}$, the following angles were measured at two points, $A$ and $B$, two miles apart:

$$
\begin{aligned}
& \text { angle } A B M=46^{\circ} 05^{\prime}, \text { angle } A B M^{\prime}=81^{\circ} 12^{\prime}, \\
& \text { angle } B A M=76^{\circ} 30^{\prime}, \text { angle } B A M^{\prime}=44^{\circ} 10^{\prime} .
\end{aligned}
$$

Compute the distance $M M^{\prime}$.

Ans. 1.34 miles.

3. $P, Q$, and $R$ are three hostile forts whose distances from each other are known. $P Q=13$ miles, $Q R=6.5$ miles, $R P=9.75$ miles. From a battery planted at $B, P R$ subtends an angle of $34^{\circ} 30^{\prime}$, and $Q R$ an angle of $23^{\circ} 45^{\prime}$. Find the range of each of the forts from $B$. Ans. $B P=9.84$ miles, $B Q=15.125$ miles, $B R=16.11$ miles.

4. Analyze the two problems stated under $(g)$ and $(h)$ in 35.1. 


\section{LESSON XXXVI - REVIEW}

1. What is meant by the logarithm of a number $N$ to the base $a$ ? What is meant by the Common system of logarithms? By the Natural system? By the Briggsian system? By the Naperian system?

2. What is meant by the characteristic of a logarithm? By the mantissa of a logarithm? By a cologarithm? Show that $\operatorname{colog} N=-\log N$.

3. Show that

$$
\log [\sqrt{ } 6 \sqrt[3]{15 / \sqrt[5]{60}}]=\frac{1}{10} \log 2+\frac{19}{30} \log 3+\frac{2}{15} \log 5 .
$$

4. Show that the mantissa of the logarithm remains unchanged if the decimal point of the number is moved. How is the characteristic affected by this change in the number? State the rule for determining the characteristic of the logarithm of a number.

5. Sketch the curves $y=3^{x}, y=3^{-x}, y=\log _{3} x$.

6. Prove that $\log _{a} N=\log _{b} N / \log _{b} a$, and $\log _{a} b \times \log _{b} a=1$.

7. Describe the curve of Damped Vibrations. Give illustrations. Give its equation and interpret the constants of this equation.

8. State the four principal cases of oblique plane triangles. Which of these cases may admit of two solutions? Why must one of the given parts always be a side if the triangle is to be completely determined?

9. Show that case I and II of oblique triangles may be solved by using the law of sines. Show that case III and IV may be solved by using the law of cosines. 
10. What is meant by a schedule of work in solving triangles? What are its advantages? What is meant by "checking the answer"? How would you check your answer in case I? In case II? In case III? In case IV?

11. State and prove the law of tangents. State the law of tangents by means of a formula involving the sides $a$ and $b$; the sides $b$ and $c$; the sides $c$ and $a$. What is the advantage of the law of tangents over the law of cosines in solving case III?

12. State and prove the formula for the radius of the inscribed circle of a triangle. Show that the area of a triangle is $s r$, where $s$ stands for half the sum of the three sides and $r$ for the radius of the inscribed circle.

13. Express the tangent of one-half an angle of a triangle in terms of the radius of the inscribed circle and the sides. In terms of the sides alone. What is the use of the half angle formulas?

14. Express both the sine and the cosine of half an angle of a triangle in terms of the sides. Do this for each of three angles.

15. Write down the formula for the area of a triangle in terms of the sides. In terms of two sides and the included angle. Prove the latter formula.

16. Show how a triangle may be solved when the three altitudes are known.

17. State and analyze Hanson's Problem. The Three Point Problem. 


\section{INDEX}

Numbers, except after Ex. (=Exercise), refer to sections

Abscissa,

Addition theorems,

for sine,

for cosine,

for tangent,

Algebra, definition of,

Algebraic expression, numbers, symbols,

Amplitude, of sine curve,

Angle, of departure, definition of, functions of double, functions of half, general value of, measure of, principal value of, Arc, length of,

Area, of closed curve, under a curve, of circular sector,

Bank clearings,

Binomial,

Briggsian logarithms,

Cartesian coördinates,

Catenary,

Centesimal measure,

Characteristic, rule for,

Checks,

Circle, equation of, construction of,

Circular functions, relations of,

Circular motion, sector, system of measure,

Cologarithm,
8.4 Common catenary

Ex. 30 (6)

24.1

24.2

24.3

24.4

1.1

1.2

3.4

1.2

21.9

21.3

6.1

17.1

24.5

24.6

25.3

$17.3 \mathrm{ff}$.

25.3

17.8

13.6

13.6

17.9

9.2

1.3

27.3

8.4

Ex. 30 (3)

17.5

27.6

27.8

31.2

14.4

14.6

$18.1 \mathrm{ff}$.

18.2

21.7

17.9

17.6

27.5

logarithms,

Complement,

Complementary angles,

27.3

17.2

19.7

Complex numbers,

3.6

21.11

10.2

8.3

8.4

8.4

18.1ff.

$18.1 \mathrm{ff}$.

24.5

24.6

23.4

18.1ff.

Cotangent, definition of,

2.1

15.7

Cubical parabola,

4.1

$15.7 \mathrm{ff}$. solution of,

Curve, mathematical,

12.2

13.5

13.6

area of closed,

Curves, catenary, circle,

cubical parabola, compound harmonic, exponential, damped vibration,

Ex. 30 (3)

14.4-14.6

15.7

21.11 .

30.1

30.5

30.2

logarithmic,

30.7

logarithmic trigonometric,

15.6

parabola.

21.10

simple harmonic,

sine,

21.1-21.6

straight line,

14.4-14.6

Damped vibrations,

30.5

Decimal system of angle measures,

Departure,

6.3

angle of,

6.1

line of,

6.1 
Displacement,

Doubtful case of triangles,

Epoch,

Equations, cubic, equivalent, $4.4,12.3,15.4,15.11$

Equations, exponential, graphic solution of, inconsistent,

in two variables,

linear,

quadratic,

roots of,

simultaneous,

simple,

straight line,

solution of,

cubic,

higher,

linear,

simultaneous,

quadratic,

quartic,

trigonometric,

Equivalent equations,4.4, 12.3, 15.4 sets of,

Exponential curves, equations,

Exponents, fractional, negative,

Extrapolation,

Factoring, def. of, binomials, monomials, trinomials,

Factor theorem,

Firing over mask,

Flight, time of,

Fourier's theorem,

Fractions,

Fractional exponents, use of

Fractional expression,

Frequency,

Function,

circular,

maximum,

minimum,

single-valued,

multiple-valued,

trigonometric,
$15.3,15.4$

15.11

30.1

28.3

21.9

32.2

21.9

4.1

28.3

15.4

15.1

$4.1,15.4$

$4.1,15.6$

4.2

15.2

4.1

14.1

4.3

15.7

15.5

15.4

15.3

15.6

15.9

25.4

2.7

2.5

8.9

1.5

1.7

1.6

1.9

1.10

6.5

6.4

21.12

3.3

2.5

2.8

1.4

21.9

13.1

$18.1,20.1,20.6$

13.2

13.2

25.2

25.2

$18.1,20.1$
Government land surveys,

8.2

Graphic methods, advantages of, uses of,

Graphic solution, of arithmetic problems, of cubic equation, of linear equation,

of quadratic equation,

11.2

15.7

15.4

of quartic equation,

15.6

of simultaneous equation, $\quad 15.3$

Graphs, of bank clearings, $\quad 9.2$

of composition,

8.10

of conversion tables,

10.2

equation of,

12.1

of marriage rate,

of price schedules,

9.2

of purchasing power,

10. $3,11.2$

simultaneous straight lines,

of straight line,

10.1

of time schedules, $\quad 10.4,11.4$

of temperature,

8.7

of unemployment,

9.2

Half-angle formulas,

Hansen's problem,

34.1 ff. Ex. 34 (7)

simple,

35.2

Harmonic

21.10

simple,

21.8

Identities, trigonometric,

21.10

Imaginary, numbers,

18.3

roots,

Inconsistent equations,

Index law,

Integers,

Integral expressions,

Integral rational,

Intercept,

Interpolation,

Interpretation of roots,

Inverse, cosine,

functions,

processes,

sine,

tangent,

Irrational expressions, numbers,

$3.3,3.5$

5.7

15.4

2.4

3.1

1.4

1.4

14.1

$8.9,22.4$

5.2

25.3

25.5

3.2

25.3

25.3

1.4

3.3

Knot, 
Lag,

Latitude, difference in,

21.10

8.1

Ex. 22 (B)

Laws of algebra and arithmetic, 3.1 of cosines, of radicals,

23.4

of sines,

2.3

of tangents,

23.3

33.1

Lead,

Line of departure, of sight,

21.10

6.1

6.1

Linear equations, solution of,

$4.1,14.2,15.4$

Linear harmonic motion, uniform motion,

Logarithmic curves,

Logarithmic trigonometric functions,

Logarithms,

Briggsian,

common,

laws of,

Naperian,

natural,

special values of,

Longitude,

15.4

21.10

21.7

30.2

30.6

27.3

27.3

27.3

27.4

30.4

30.4

27.5

8.1

Mantissa,

Mariner's compass,

Marriage rate, graph of,

Ex. 22 (B)

Mask, firing over,

9.2

6.5

Mathematical curves,

12.2

Maximum,

13.2

problems, in

test for,

13.4

13.3

29.1

Mensuration,

Mil,

Minimum, problems in, test for,

13.2

13.4

13.3

1.3

Monomial,

25.2

Multiple-valued functions,

Naperian logarithms,

Natural functions, logarithms, numbers, system of angle measure,

Negative angles,

Negative exponents, numbers,

20.2

30.4

3.1

17.6

19.6

2.7
Numbers, algebraic,

3.4

3.6

3.3

$3.3,3.5$

3.3

3.3

3.3

3.3

3.4

Ordinate,

8.4

Origin,

8.4

Parabola, cubical,

Period,

15.7

21.9

Periodic curve, $\quad 21.3$

Periodicity, $\quad 19.2$

21.3

Phase,

$\mathrm{Pi}(\pi)$,

Polynomial,

Price lists, schedules,

Principal value,

Proportional parts,

21.9

17.7

1.3

11.3

10.3

25.3

22.4

Quadratic equations, $\quad 8.5$ character of roots, $\quad 5.1$ completing the square, $\quad 4.7$ general form of, general solution of, graphic solution of,

Quotient relations,

$15.9,15.10$

18.2

Radian measure,

Radicals, laws of,

Range,

Rational, definition of,

Real numbers,

18.2

Reciprocal relations,

8.4

Rectangular coördinates,

19.8

Reduction formulas,

18.2

Relations, fundamental,

18.2 quotient, reciprocal,

18.2

Relations, square,

18.2

3.3 Roots of an equation, 
Schedule of work,

Secant, definition of,

Sector, area of,

Sexagesimal system,

Sight, line of,

Significart figures,

Simple equations,

Simple harmonic curves, motion,

Simultaneous equations, graphic solution of,

Sine, definition of, curve,

of double an angle,

of half an angle,

law of,

Sine curve,

amplitude of, periodicity of, wave-length of,

Single-valued functions,

Slope,

Solution of equations,

Square, completing the,

Square relations,

Square root,

Straight line, construction of, equation of, graphs,

Subtraction theorems,

Supplement,

Tangent, definition of, of half an angle, law of, \begin{tabular}{l|lr}
31.3 & Temperature graph, & $\mathbf{8 . 7}$
\end{tabular}

18.1, 20.1 Three-inch field gun, $\quad 6.3$

17.9 Three-point problem, 35.3

17.3 Time of flight, $\quad 6.4$

6.1 Time schedules, $\quad 10.4$

22.10 Train schedules, $\quad 11.4$

4.1 Trajectory, $\quad 6.1$

21.10 Transcendental numbers, $\quad 3.4$

21.8 Triangles, area of, 23.8

15.2-15.4 arithmetic solution of, 23.1

15.5-15.8 logarithmic solution of, 31.1

18.1, 20.1 parts of, 22.1

21.1, 21.2 Trigonometric equations, $\quad 25.4$

24.5 involving inverse functions, 25.5

24.6 Trigonometric functions, 18.1, 20.1

23.3 inverse, 25.1

$21.1,21.3 \quad$ lines, $\quad 20.6$

21.3 logarithmic, $\quad 30.6$

21.3 periodicity of, 19.2

21.3 signs of, $\quad 19.1$

25.2

14.1

4.3

4.7

18.2

2.1

14.3

14.1

10.1

24.1

17.2

variations of,

20.7

of $45^{\circ}$,

20.3

of $30^{\circ}$ and $60^{\circ}, \quad 20.4$

of $0^{\circ}$ and $90^{\circ}$,

20.5

Trigonometric identities, $\quad 18.3$

Trinomials, $\quad 1.3$

Unemployment, graph of, $\quad 9.2$

Uniform circular motion, $\quad 21.7$ linear motion,

Vickers-Maxim gun,

$18.1,20.1$

34.1

Wages, graph of,

9.1

33.1 Wave-length, 
. 


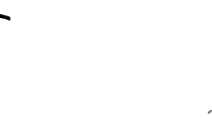




\section{.}



$\therefore \quad 1-y^{2} y^{3}$

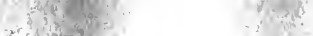




\section{DAY USE}

\section{RETURN TO DESK FROM WHICH BORROWED}

\section{LOAN DEPT.}

This book is due on the last date stamped below, or on the date to which renewed.

Renewed books are subject to immediate recall.

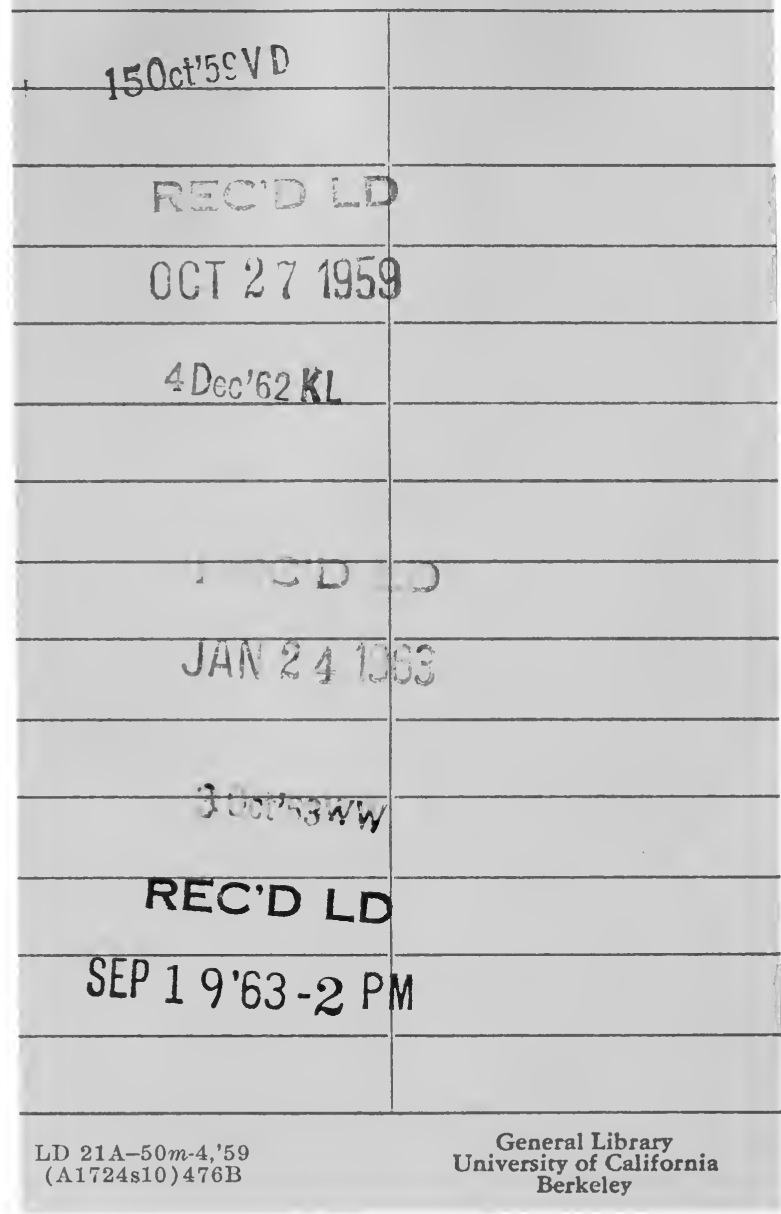




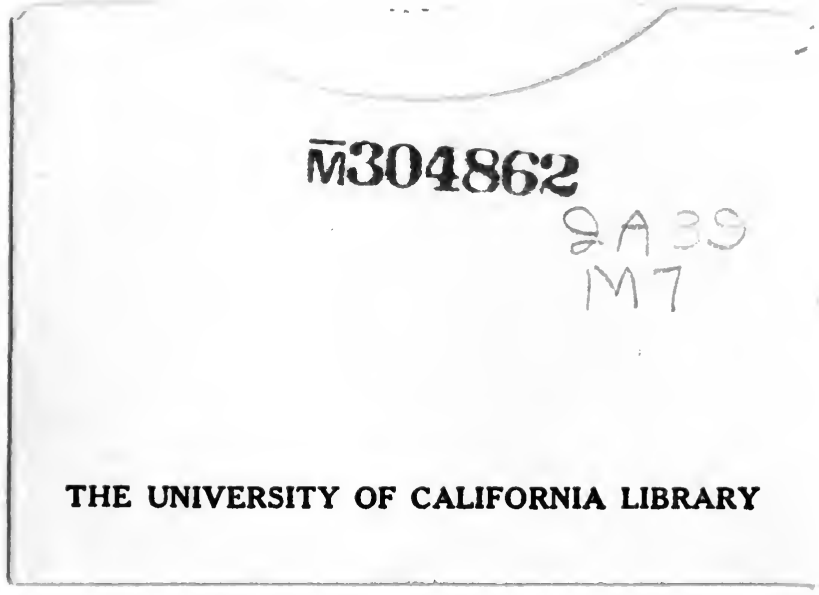


